\title{
The Ends of Uncertainty: Air Quality Science and Planning in Central California
}

\author{
Prepared By: \\ James Fine \\ Atmospheric Science Department \\ Environmental Energy Technologies Division \\ Lawrence Berkeley National Laboratory \\ Berkeley, CA 94720-1740
}

Contact person at LBNL:

Dr. Nancy J. Brown

Phone: 510-486-4241

Fax: 510-486-7303

e-mail: njbrown@lbl.gov

\author{
Author: \\ Dr. James Fine \\ Department of Environmental Science \\ University of San Francisco \\ 2130 Fulton Street \\ San Francisco, CA 94117-1080 \\ Phone: 415-422-6159 \\ Fax: 415-422-6387 \\ e-mail: jdfine@usfca.edu
}

September 2003 
The Ends of Uncertainty:

Air Quality Science and Planning in Central California

\author{
By \\ James David Fine \\ B.S. (University of Pennsylvania) 1989 \\ M.S. (University of California at Berkeley) 1998
}

A dissertation submitted in partial satisfaction of the requirements for the degree of

Doctor of Philosophy

in

Energy and Resources

in the

GRADUATE DIVISION

of the

\title{
UNIVERSITY OF CALIFORNIA AT BERKELEY
}

Committee in charge:

Professor Elizabeth Deakin

Professor Gene Rochlin

Professor Robert Harley

Dr. Nancy Brown

Spring 2003 


\begin{abstract}
The Ends of Uncertainty:

Air Quality Science and Planning in Central California
\end{abstract}

By

James David Fine

\begin{abstract}
Air quality planning in Central California is complicated and controversial despite millions of dollars invested to improve scientific understanding. This research describes and critiques the use of photochemical air quality simulation modeling studies in planning to attain standards for ground-level ozone in the San Francisco Bay Area and the San Joaquin Valley during the 1990's. Data are gathered through documents and interviews with planners, modelers, and policy-makers at public agencies and with representatives from the regulated and environmental communities. Interactions amongst organizations are diagramed to identify significant nodes of interaction. Dominant policy coalitions are described through narratives distinguished by their uses of and responses to uncertainty, their exposures to risks, and their responses to the principles of conservatism, civil duty, and caution. Policy narratives are delineated using aggregated respondent statements to describe and understand advocacy coalitions.
\end{abstract}

I found thar models impacted the planning process significantly, but were used not purely for their scientific capabilities. Modeling results provided justification for decisions based on other constraints and political considerations. Uncertainties were utilized opportunistically by stakeholders instead of managed explicitly. Ultimately, the process supported the partisan views of those in control of the modeling. Based on these findings, as well as a review of model uncertainty analysis capabilities, I recommend modifying the planning process to allow for the development and incorporation of uncertainty information, while addressing the need for inclusive and meaningful public participation.

By documenting an actual air quality planning process these findings provide insights about the potential for using new scientific information and understanding to achieve environmental goals, most notably the analysis of uncertainties in modeling applications. Concurrently, needed uncertainty information is identified and capabilities to produce it are assessed. Practices to facilitate incorporation of uncertainty information are suggested based on research findings, as well as theory from the literatures of the policy sciences, decision sciences, science and technology studies, consensus-based and communicative planning, and modeling. 


\section{Table of Contents}

EXECUTIVE SUMMARY ........................................................................................ 1

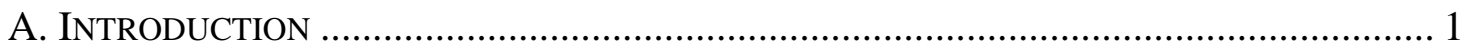

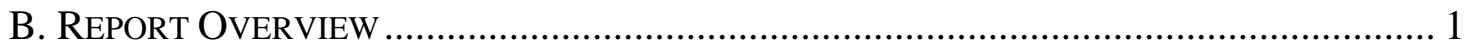

C. Planning, Modeling And UnCERTAinty (Chapter II) ......................................... 2

D. Photochemical Air Quality Simulation Models (Chapter III) ....................... 2

E. Case Study: Central California Air Quality Planning (Chapters IV - VIII)4

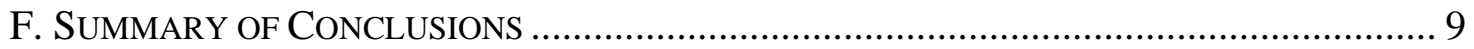

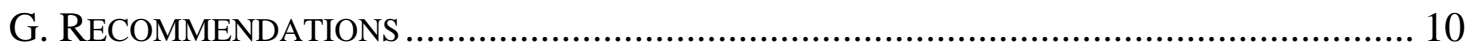

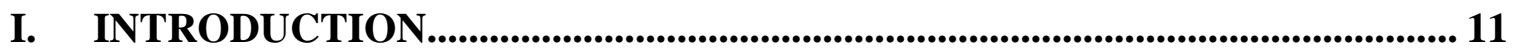

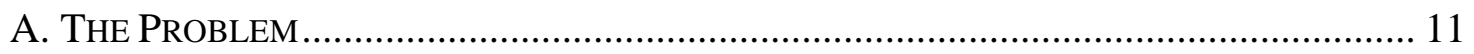

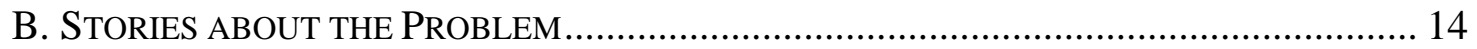

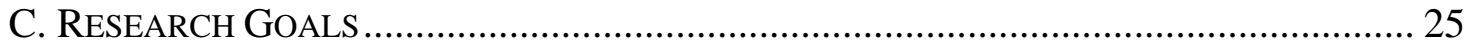

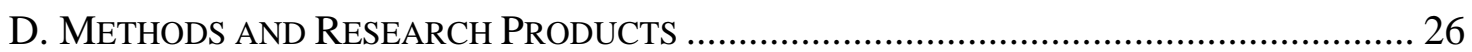

II. PLANNING, MODELING AND UNCERTAINTY ...................................... 27

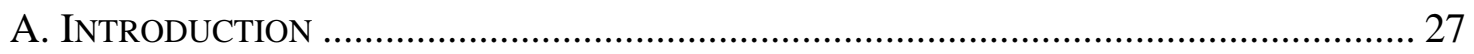

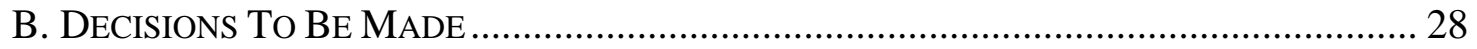

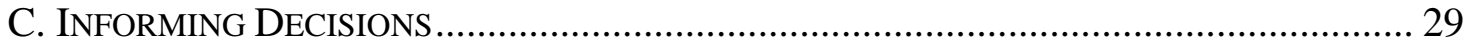

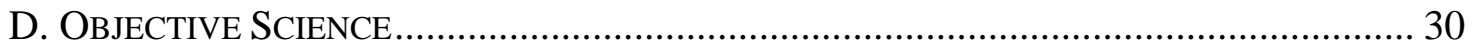

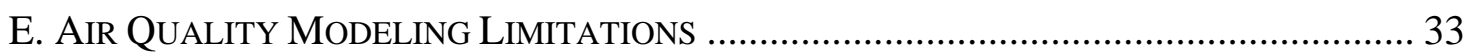

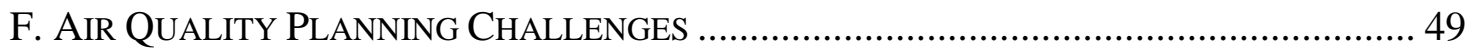

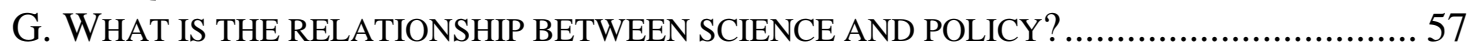

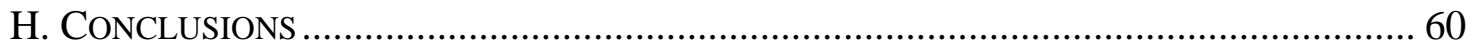

III. PAQSM AND THEIR UNCERTAINTIES ............................................. 62

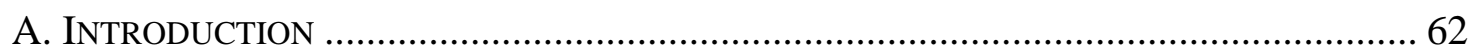

B. OZONE COMPLEXITY AND MODELS..................................................................... 62

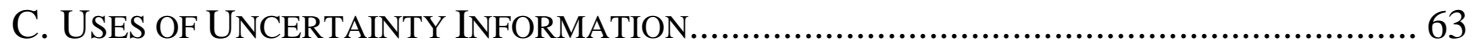

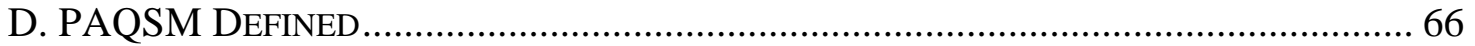

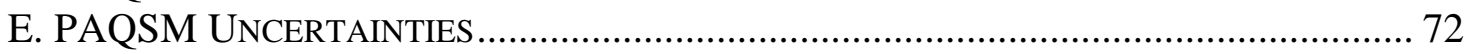

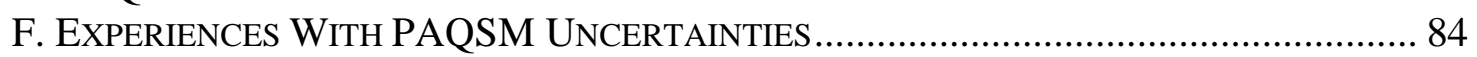

G. FRAMEWORK FOR UNCERTAINTY ANALYSES .................................................... 88

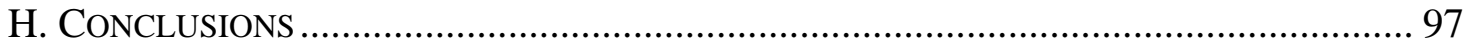

IV. CENTRAL CALIFORNIA AIR QUALITY ..........................................99

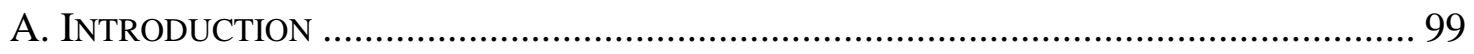

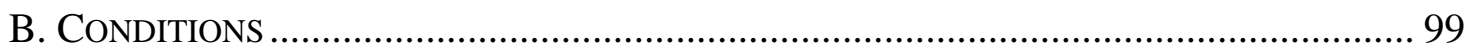

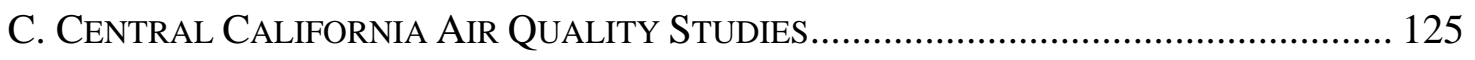

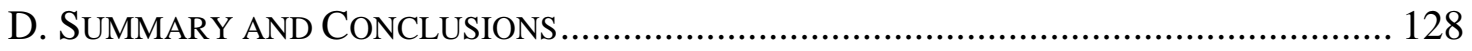

E. ApPendix IV-A: CENTRAl CALIFORniA Air Quality Study SPONSORS ............. 130 
V. CENTRAL CALIFORNIA AIR QUALITY MODELING 132

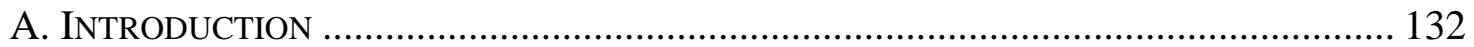

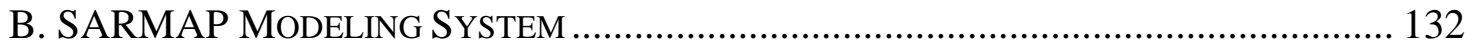

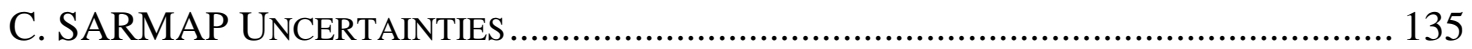

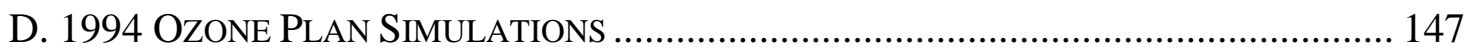

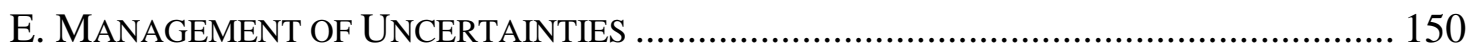

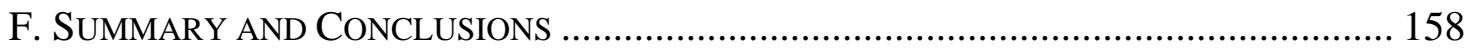

VI. CENTRAL CALIFORNIA PLANNING PART I - DESCRIPTION .......... 159

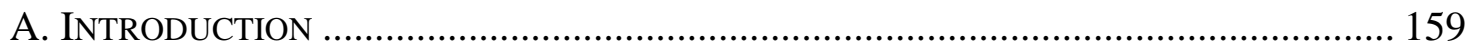

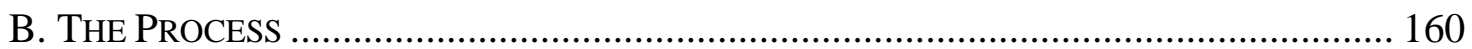

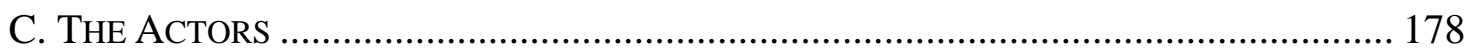

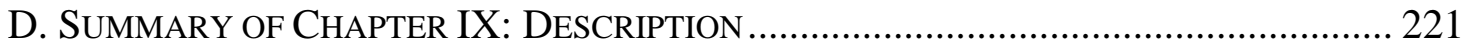

VII. CENTRAL CALIFORNIA PLANNING PART II - DEBATES AND

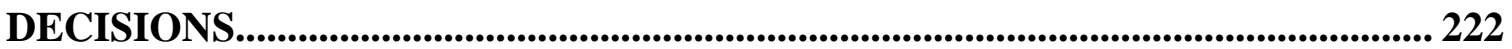

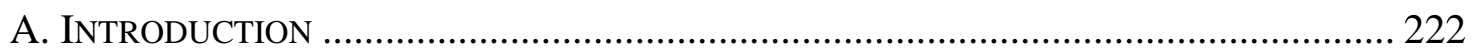

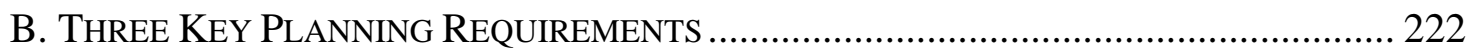

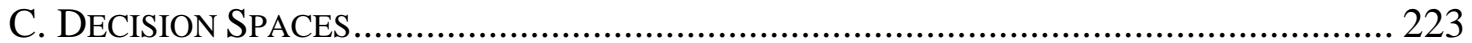

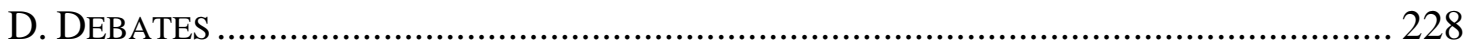

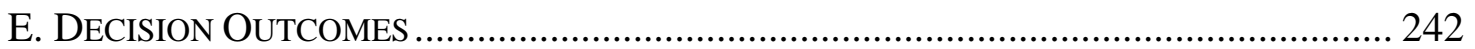

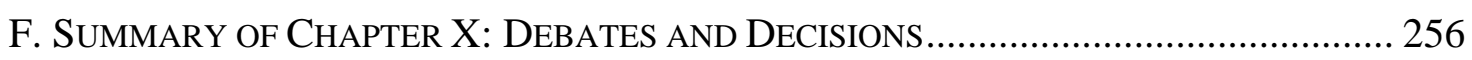

VIII. CENTRAL CALIFORNIA PLANNING PART III - USES OF MODELS

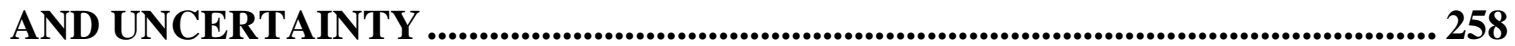

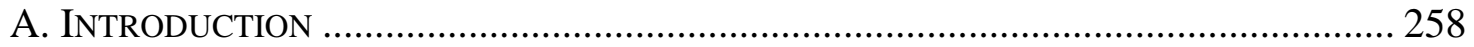

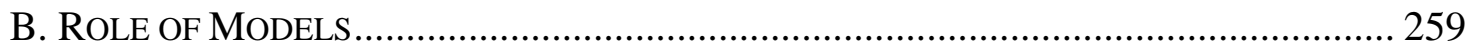

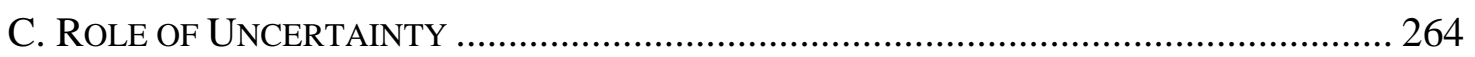

IX. RECOMMENDATIONS...................................................................... 284

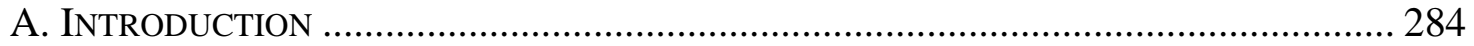

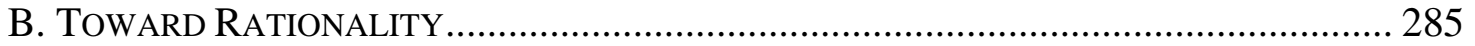

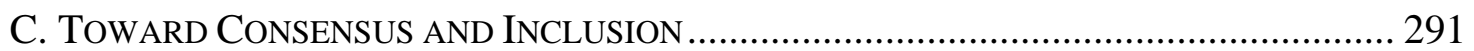

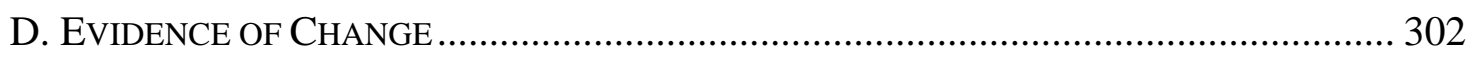

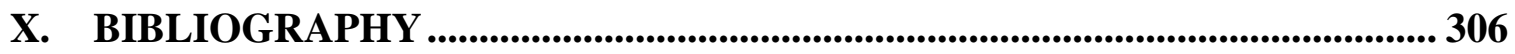

APPENDIX A: CASE STUDY METHODS AND PROCEDURES........................ 330

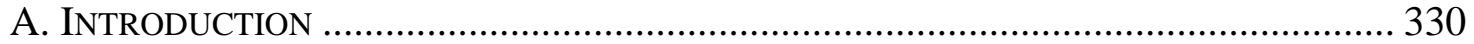

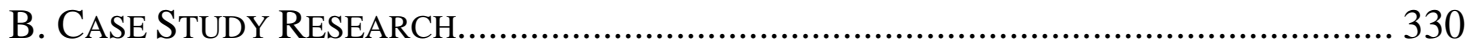

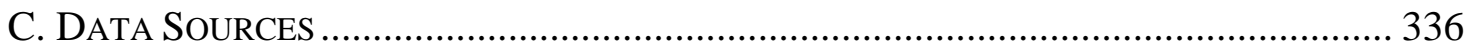

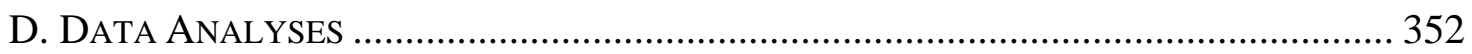

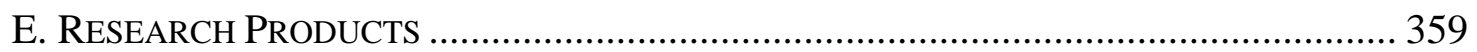

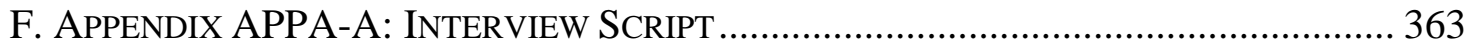

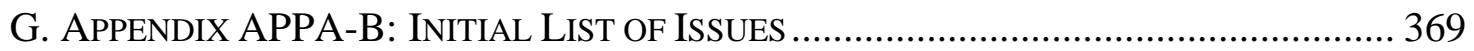




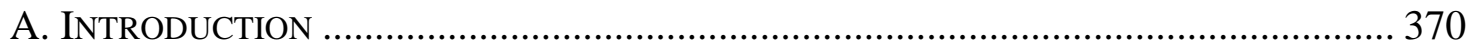

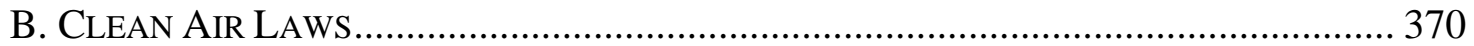

C. Air Quality MANAGEMENT AgencieS ............................................................... 378

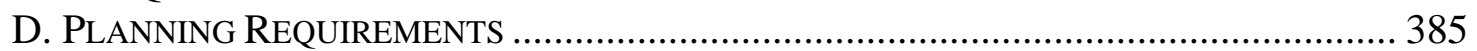

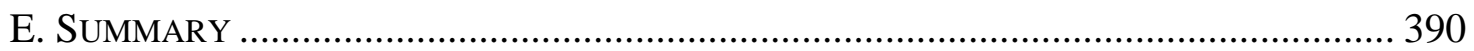

APPENDIX C: CENTRAL CA SOCIOECONOMIC SETTING.............................. 392

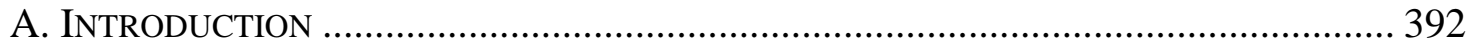

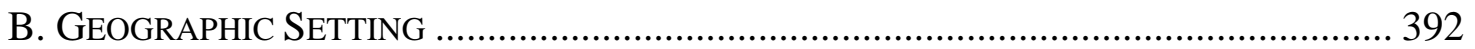

C. DEMOGRAPHIC AND SOCIOECONOMIC SETTING ….................................................... 397

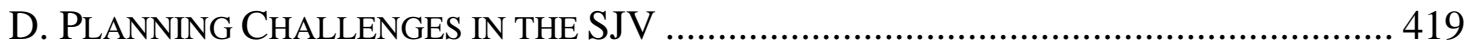

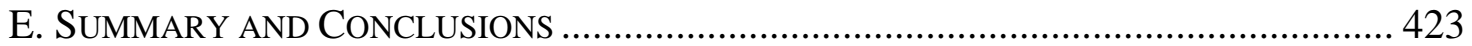

\section{Index of Respondents Quoted}

DaMassa. $184,342,345$

DeMandel $20,170,173,191,214,260,346,349$

Howekamp $151,192,193,204,341,345$

Jones 219, 243

Leong.....

McNerny. 166, 167, 168, 169, 170, 172, 173, 174, 185, 251, 346, 348

Nester $190,220,342,346$

Scheible 214, 346

Shipp.... 172, 191, 203, 204, 244, 342

Sweet 217, 276

Terry...... $150,170,186,188,189,297$

Tranrikulu

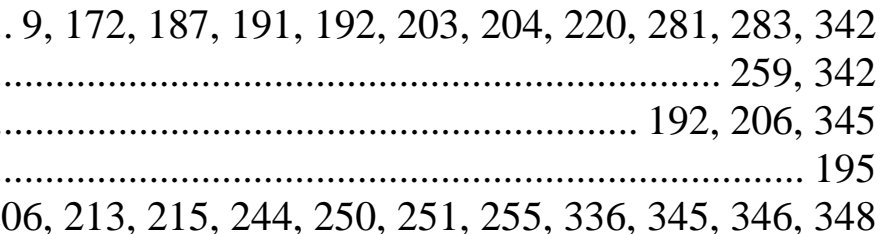

Wang

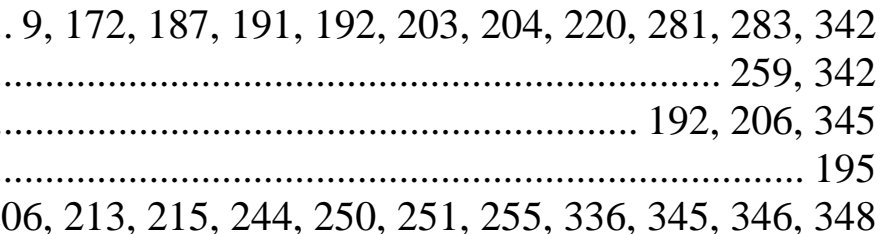

Weisser

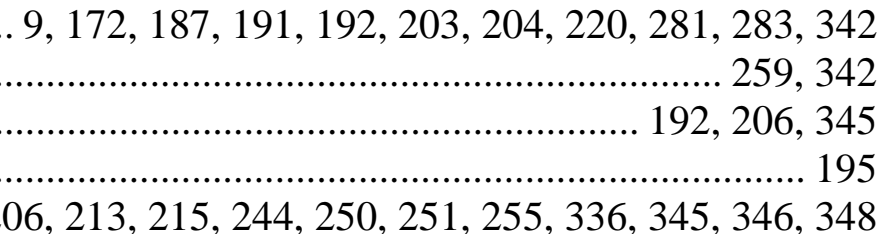

Ziman 


\section{EXECUTIVE SUMMARY}

\section{A. Introduction}

This report is based on a dissertation by James Fine, The Ends of Uncertainty: Air Quality Science and Planning in Central California, which was filed with the University of California at Berkeley Energy and Resources Group on May 23, 2003. The research involved two primary tasks:

1. Description and critique of the uses of simulation models and the management of uncertainties in modeling in air quality planning. This task was completed using a case study of Central California in the 1990's. In addition to description and critique, the research produced prescriptions for modifying the planning process to allow for the development and incorporation of uncertainty information, as well as the meaningful participation of all stakeholder groups.

2. Review of photochemical air quality simulation model uncertainties and uncertainty analysis capabilities to examine what model uncertainty information can and should be produced to assist air quality planners. This task produced journal article to be published in the Annual Review of Energy and the Environmental, V28 (November 2003). Chapter III in this report is based upon and extends the article.

This research was supported by the Assistant Secretary for Fossil Energy, Office of Natural Gas and Petroleum Technology, through the National Petroleum Technology Office under U.S. Department of Energy Contract No. DE-AC03-76SF00098.

\section{B. Report Overview}

The first chapter introduces several "stories" about "the problem” using narratives developed through this research. and then presents questions motivating the report. Chapter II reviews the theoretical literature about the challenges of model-based decision-making. Photochemical air quality simulation models are described in Chapter III in terms of their increased regulatory application, sources and magnitudes of uncertainties, and experiences with methods to evaluate those uncertainties. These several chapters provide the theoretical and practical groundwork for exploring the question of the uses of models in an actual case: Central California air quality planning in the 1990's.

Five chapters cover the case's air quality conditions, modeling application, planning process description, debates and decisions, and, finally, uses of models and uncertainty. Chapter IV summarizes air quality conditions, emissions sources and coordinated air quality studies in Central California. The modeling system used in the case, as well as the treatment and communication of associated uncertainties, is discussed in Chapter V. The social aspect of the air quality planning process are described in Chapter VI, whereas Chapters VII and VIII present key debates and decisions, and summarize the roles of models and their uncertainties, respectively. Last, Chapter IX offers recommendations for using a risk management approach to air quality decision-making and for meaningful inclusion of all stakeholders in the planning process. In addition, three appendices 
describe the case study approach employed (Appendix A), and provide more detail about the regulatory (Appendix B) and socioeconomic (Appendix C) settings of the case.

The remainder of this Executive Summary summarizes findings associated with specific research questions and is organized by chapter.

\section{Planning, Modeling and Uncertainty (Chapter II)}

The literature about managing uncertainty in decisions, model-based decisionmaking, consensus-based and communicative planning, and social aspects of science, support three logical positivist claims:

1. Objective information is needed for environmental decision-making.

2. Scientific research provides information per the needs of decision-makers.

3. Modeling research is uniquely capable of producing some needed information.

Building upon these claims, and drawing from the lessons of the policy sciences, and the social studies of science and technology, are two conclusions:

4. Modeling applications are not purely objective, due to limitations of uncertainty, opacity and subjectivity.

5. Air pollution, like many other environmental problems, cannot be managed solely through scientific research because it involves significant, inequitably-shared risks and burdens, competing values, and contested science.

In light of the observation that clean air lawmakers placed great faith in the objective and informative capabilities of scientific research, including modeling, two responses to the inability of science to provide reliable information without controversy are suggested:

A. Modeling and other research results should be presented and used in ways that clarify and mitigate their limitations.

B. Emphasis should be placed on planning processes that consider explicitly non-technical components of the problem through consensus-based, pluralistic means that employ a set of principles for generating and communicating information.

\section{Photochemical Air Quality Simulation Models (Chapter III)}

This chapter addresses three research questions:

- What are the potential uses of explicating model uncertainty information?

- How might potentially useful uncertainty information be produced and utilized?

- What technical factors impede the integration of uncertainty information into air quality planning decisions? 
Fine et al. (2003) present conclusions about evaluating uncertainties in PAQSMs.

1. Uncertainties pervade the use of models. Consequently, a range of model estimates may be anticipated for a given set of inputs and their associated uncertainties. Thus, estimates of uncertainties should be made and factored into processes involving model-based decision-making associated with air quality issues.

2. Methods available today that are truly useful either partially address the estimation need or focus on a defined, limited part of the problem. Unfortunately, no method is now available for estimating uncertainty in modeling that is comprehensive in scope.

3. A comprehensive method for analyzing uncertainty information would (a) propagate uncertainty from each component of the modeling system through the system into an estimate of uncertainty associated with model output, (b) elucidate bias, and (c) account for variability. The method would also synthesize and integrate results from the various methods employed to estimate uncertainty comprehensively. The products of a comprehensive uncertainty assessment would be distributions or probabilistic statements characterizing the uncertainty of model estimates.

4. Developing a comprehensive approach to uncertainty analysis would be very valuable. Its feasibility should be assessed. Such an approach may not be possible, since nothing in the literature suggests so.

5. In practical applications, visual inspection of plots of concentration versus time for pollutant species of interest provides adequate information to determine if model performance is sufficiently acceptable to merit proceeding with comprehensive uncertainty assessment. In many cases, model performance is wanting. Where performance is unacceptable, major flaws in the model should be corrected prior to obtaining uncertainty information.

6. Sensitivity/uncertainty analysis is by far the most frequently used method for estimating uncertainty. Its focus is on the response of a dependent variables to changes in inputs. Where the response is significant, uncertainty is likely to be important; where the response is small, the converse is expected. Sensitivity analysis does not address bias as an element in uncertainty explicitly. Generally, it is not suited for this use, as the main assumption made is that the model is substantially correct in its representation of reality.

7. As the key element of a comprehensive assessment of uncertainty, the development and application of methods for identifying, estimating, and reducing biases (i.e., mitigating or eliminating flaws in model representations) should be made a priority. This includes determining when bias is present, how to identify it, and what to do when it corrupts modeling results. Through assessment of bias, model formulation may be improved to increase the probability that the model is performing acceptably well for the right reasons and that modeled sensitivities are reliable. Examining the issue of potential bias typically requires case-specific procedures. 
8. In addition to the evaluation of bias, natural and human-induced variability should receive attention in the comprehensive estimation of uncertainty. Some deterministic modeling formulations may simulate well-characterized stochastic processes using statistical sampling techniques, whereas others, such as those used to derive meteorological inputs, are incapable of simulating stochastic processes. The appropriateness and feasibility of developing stochastic models merits attention because they are a potentially attractive means for incorporating variability.

9. Designing a comprehensive approach to uncertainty assessment that can be implemented and that addresses bias and variability requires a major research effort. To date, no such effort has been formulated, let alone undertaken. Rather, the focus has been on portions of the problem, in the absence of a more encompassing plan that might foster a more integrated research program design.

Building upon the above findings are the following conclusions based on the case study presented in subsequent chapters:

- There is a commitment to uncertainty assessment in the research arena, but a lack of commitment to assess it comprehensively in regulatory applications. Performance requirements are inadequate to identify bias or to generate explicit statements about the uncertainties in model results.

- Uncertainty assessment capabilities are currently insufficient to provide to policy-makers detailed, synthesized, useful uncertainty information

- It is feasible to develop available methods further, thereby gaining experience and improving capabilities. Doing so requires a commitment to developing and maintaining an adequate observational database. Currently, commitment is lacking to support such an effort. However, progress is being made. There is anticipated regulatory guidance from EPA on how to model to demonstrate attainment of the 8-hour standard. Initial drafts suggest it will be a promising step forward. Observational databases are being improved by ongoing field studies, such as the Central California Ozone Study and the California Regional Particulate Air Quality Study, thereby providing more data to input into models and to use for modeling performance evaluation.

\section{E. Case Study: Central California Air Quality Planning (Chapters IV - VIII)}

Several chapters and appendices provide context about the case, including the regulatory, socioeconomic, and air quality conditions, and describe uncertainties associated with the modeling application used to support ozone attainment planning and the assessment of regional-scale pollutant transport. Noteworthy conclusions address the research questions:

- How does air quality planning work in practice? 
- What are the dominant stories and disagreements associated with air quality planning in Central California?

- How are models and uncertainty used in current air quality planning practice?

- What legal, socioeconomic and political factors impede the integration of uncertainty information into planning decisions? (The research question of technical capability to generate uncertainty information is addressed in Chapter III).

\section{Setting}

Modeling for Central California air quality planning is an example of what the science and technology studies literature calls a post-normal problem at the interface of science and policy. The modeling is uncertain and contested, decision stakes are enormous, and those with differing values and risks disagree about appropriate actions. Science alone cannot resolve their disagreements.

Other findings from the setting chapters are that San Joaquin Valley and Bay Area where quite different in the 1990's and continue to be so. Differences are due largely to divergent histories of air quality conditions, planning efforts, science studies, and sociopolitical setting.

Although air quality has improved since the 1970's, progress over the past decade is difficult to detect because the number of days violating state and federal ozone standards has not declined significantly since 1990. Furthermore, new air quality standards for fine particulate matter and ozone concentrations averaged over 8 hours, as well as regional haze, provide additional impetus for emissions reductions. These needs are made all the more pressing by an anticipated doubling of the SJV population by 2040 .

In the Bay Area, there is general agreement amongst scientists that peak ozone formation is limited by VOC emissions and that local emissions influence air quality in the SJV. The science provides less definitive control strategy guidance in the SJV, where air quality conditions are far worse. Neither precursor pollutant is obviously the limiting reagent in peak ozone formation in the SJV. Also, the environmental community has only recently begun to organize participation in SJV air quality planning, whereas it has been quite active in the Bay Area for decades. These considerations have implications for getting emissions reductions from previously uncontrolled sources, notably farming equipment and diesel trucks, and further reductions from passenger vehicles. It remains to be seen if political will in the SJV is sufficient to achieve the reductions necessary to meet air quality goals.

\section{Uses of PAQSMs and Their Uncertainties}

Interviews and archival documents provide the data to describe the Central California air quality planning process in the 1990's. These data provided rich narratives about the 
case told by those who know it best. Several findings are notable that pertain to the facts, social networks, controversies and narratives:

- The process evolved from being technocratic in the 1980's to one with characteristics of partisan, technocratic and consensual processes by the 1990's. Expert simulation models are now a central element of the process, but do not determine decisions. Models are not used purely for their scientific capabilities; they are also used for partisan purposes, such as supporting predetermined policy positions. The interagency, stakeholder-led and -funded San Joaquin Valley Air Quality Study was “cooperative” but not truly consensus-based because some stakeholders, notably environmental and community advocates, were not represented.

- Three networks are diagrammed to show organizations involved in the production of the 1994 Ozone Plan. Network analyses reveal how control over problem definition and the production and communication of information provide influence over the process. One route of that influence is modeling.

- The influence of any individual organization is limited. No single agency has complete authority or jurisdiction over the emissions sources causing degraded air quality in Central California.

- Four factors define and constrain the way models are used for decision-making in the planning process: legal and regulatory requirements, time, science, and political will. Given myriad constraints, respondents agreed that modeling results do not drive planning decisions. The planners' challenge is to conduct a modeling demonstration using the "best available" information and within timelines that supports emissions control strategies constrained by political will. Expressed as "feasible" emissions controls, political will is determined by technical capability, cost effectiveness, and regulators' perception of the public's willingness to accept emissions controls.

- Where the respondents differ the policy narratives come into focus. Here, narratives pertain to the different stories told about the process. Narratives are distinguished by emphasized or discounted facts and by interpretations of available, yet uncertain, scientific information. The narratives disagree about what policy decisions are indicated by modeling studies and accompanying evidence, the reliability of modeling results and implied control strategies, and what should be done in response to uncertainty, including communicating it to the public, basing research efforts upon it, and hedging against it in decision-making.

- Three policy narratives delineated are: (1) Feasibility, (2) Science/Efficiency, and; (3) Control All. Their key arguments are summarized in Table XII-A. 
Table XII-A: Narratives and Their Opinions

\begin{tabular}{|l|l|}
\hline Narrative & Opinions \\
\hline Feasibility & $\begin{array}{l}\text { Problem: Ozone, particulate matter, visibility, toxics, } \\
\text { environmental justice and politics } \\
\text { Response: Reduce all emissions “feasible” }\end{array}$ \\
& $\begin{array}{l}\text { Models: Too uncertain for precise plans } \\
\text { Control Debate: Overwhelmed by other air quality concerns }\end{array}$ \\
\hline $\begin{array}{l}\text { Science and } \\
\text { Efficiency }\end{array}$ & $\begin{array}{l}\text { Problem: Ozone } \\
\text { Response: Minimize social costs equitably }\end{array}$ \\
\hline Control All Use to delineate controls \\
& $\begin{array}{l}\text { Control debate: Essential issue } \\
\text { unduly influenced by the political and economic concerns }\end{array}$ \\
& $\begin{array}{l}\text { Response: Reduce all emissions } \\
\text { Models: Opaque and not trustworthy } \\
\text { Control Debate: Irrelevant }\end{array}$ \\
\hline
\end{tabular}

Findings about the roles of models and uncertainty in the case are:

Decision-Making with Models

Modeling affects planning in three ways: (1) modeling is a central focus of planning, (2) stakeholders must engage models to engage planning, and (3) control of modeling provides influence in decision-making.

Models do not determine decisions. Instead, they are used to justify decisions made based on other factors, notably deadlines, legal and regulatory requirements for using the "best available" science and meeting the letter of the law, and political will.

\section{Planning with Models}

In the late 1970's, modeling became an important route to participating in or defending air quality planning decisions. Today, modeling remains a central focus of air quality planning. The application of the SARMAP modeling system to produce the 94 Ozone Plan framed the debate by providing justification for planned emissions controls. To participate in planning, it was necessary to engage the modeling. Those in control of the modeling gained the upper hand in decision-making.

These findings have great importance for the project of generating information about the uncertainties in modeling. Since models do not determine control decisions, it is wrong to assume that modeling results will be used by decision-makers just because they are more complete. Findings about the use of uncertainty build upon those about modeling.

\section{Discourses of Uncertainty}

All narratives speak of the significance of uncertainty attending air quality planning and the associated modeling. Consequently, one way that uncertainty is used is to form 
the dominant policy narratives: science/efficiency and feasibility. The feasibility narrative claims that modeling results are too uncertain to identify a single-precursor (i.e., $\mathrm{NO}_{\mathrm{x}}$ or VOCs) control strategy; instead suggesting that a precautionary approach is to control both types of emissions. The science/efficiency narrative says that precaution entails awaiting costly controls until the science is more definitive.

The Bay Area refinery and Kern County steam generate $\mathrm{NO}_{\mathrm{x}}$ emissions control decisions indicate how modeling is used to justify decisions, when it can be shown to do so, and how the model uncertainties are both the bane and bounty of decision-makers and stakeholders. In the former example, modeling was used to link Bay Area emissions with poor air quality in the SJV. To mitigate pollutant transport, CARB called for $\mathrm{NO}_{\mathrm{x}}$ controls on BA refineries. When the same modeling indicated that the $\mathrm{NO}_{\mathrm{x}}$ controls offered uncertain benefits in the SJV and possible ozone disbenefits locally (i.e., in the SF Bay Area), CARB rebuked the findings of its own technical staff by noting the modeling was uncertain.

In Kern County, the same modeling system was used by petroleum interests to indicate that steam generators used for petroleum production in western Kern County did not need to implement $\mathrm{NO}_{\mathrm{x}}$ controls for the region to meet the federal 1-hour ozone standard.

\section{Opportunistic Use of Uncertainty}

Stakeholders use uncertainties opportunistically. Regulators at government agencies use it to pursue a policy of controlling all emissions feasible, per the feasibility narrative. When the regulated industry believes that proposed controls will be ineffective, it calls for uncertainty reduction, first, and then control implementation only when justified by the science. Oftentimes, industry's argument causes a delay in control measure implementation.

\section{Assumptions About Uncertainty}

There is a tendency to assume that quantifying uncertainty will mean more controls are necessary. If so, the logic goes, and there are already too few control measures available, then uncertainty information is irrelevant. While it is true that planning for contingency controls is one way to address uncertainty, the respondents often did not recognize that the uncertainties may work both ways. Uncertainty assessment may provide the confidence needed to pursue a single precursor strategy, thereby mitigating the need to find more emissions controls.

\section{Uncertainty Communication}

Uncertainties in planning are not communicated to the public clearly, and uncertainties are not managed explicitly in the decision-making context. For example, the 94 Ozone Plan told two incongruent stories about uncertainty. First, the scientific bases of the plan was said to be uncertain and evolving. Second, the plan used modeling to show that anticipated controls will meet ozone goals, but did not account for known uncertainties, such as an erroneous and incomplete emissions inventory. The control strategy did not attempt to manage the acknowledged uncertainties. 


\section{Model Performance Evaluation}

There is little indication that the uncertainties are considered formally or rigorously in air quality decision-making. Models' uncertainties are only evaluated so that performance can be shown to meet criteria, even though respondents agree that the criteria do not provide assurance that the model is adequately reliable.

Uncertainty information accompanying modeling results is lost or truncated as it passes from modelers to planners to executive policy-makers. In turn, decision-makers believe they internalize their knowledge of the uncertainties. Nonetheless, the research process continues toward improving models and emissions inventories in anticipation of future planning cycles. This demonstrates that there is a desire to reduce uncertainties and, presumably, to make decisions based on better understanding.

In sum, the prospects for deterministic use of models are limited, even though that is how they will best serve the objective needs of planners. Similarly, there is little room in the process for incorporating information about models' uncertainties. Uncertainty currently serves the subjective goals of those with decision-making authority, such as pursing a policy of reducing all emissions "feasible."

That uncertainty is not considered formally or rigorously because it is not provided is putting the cart before the horse. The constraint is not lacking methods for producing uncertainty information or the observational database necessary to employ the methods. Instead, it is the human component, the political factors that combine with other constraints imposed externally, such as planning deadlines, that obviate the use of models and the pursuit of their uncertainties.

\section{F. Summary of Conclusions}

The literature concludes that models are neither objective nor certain decision tools. Therefore, modeling limitations should be addressed, communicated and managed explicitly in decision-making. Currently, modeling uncertainties are not evaluated comprehensively, nor is there experience with methods to do so. Capabilities remain limited for uncertainty assessment, synthesis and communication. There is little regulatory or policy-making impetus to improve capabilities, including a lack of support to provide more robust observational databases.

The case study exemplified that models are not used purely for their scientific capabilities, and that uncertainty information is not pursued for myriad reasons. Although more information is considered useful and desirable, uncertainty is not managed explicitly in emissions controls decisions. One reason uncertainties are not rigorously considered is the lack of available information, hence the need to continue with efforts to provide it. CARB's Deputy Executive Officer, Lynn Terry , says “Any information is welcome" and information, if made available, might be used. In the interim, models are considered to be too coarse to "micromanage" air quality and, although uncertainty is relevant, those in control of decisions do not see the need for its explication. In practice, however, policy-makers do not seek uncertainty information. Consequently, policy-makers do not inspire modelers to improve the practice of uncertainty evaluation and communication. 


\section{G. Recommendations}

If uncertainty information is not produced, it will certainly never be usable. Federal, state and local air quality managers need to take two steps toward generating and managing uncertainty information. First, they should require more extensive evaluation of uncertainties in modeling. Second, they should require the translation of evaluation results to policy-makers. Though experience with doing so is currently wanting, there are reasons to be optimistic. Planners, modelers and executive-level policy makers comprise a considerable brain trust with several decades of experience in California alone. In aggregate, they have an ever-improving understanding of the issues and take obvious pride in a job well done, especially when the health of humans and ecosystems is at stake.

The specific recommendations address two objectives: (1) rational and (2) inclusive participatory decision-making. Toward the first objective, recommendations suggest approaches for developing qualitative and quantitative assessments of uncertainties in modeling, as well as other sources of uncertainty, and the translation of that information to decision-making using a risk management paradigm. This latter component involves the use of probabilistic assessments and statements about the likelihood of outcomes, both desirable and negative, associated with alternatives.

Recognizing that reliance of scientific information, such as modeling results and risk assessment, may impede the participation of lay stakeholder groups with a legitimate stake in decisions, two sets of recommendations are suggested to facilitate inclusive participation, and to make that participation meaningful. These recommendations involve including stakeholder groups early in the process and providing them with expert representation, as necessary, to understand and critique the scientific bases of decisions. 


\section{INTRODUCTION}

This research examines and critiques the uses of simulation models in air quality

planning and suggests ways to incorporate information about model uncertainties into

planning decisions.

\section{A. The Problem}

Worldwide an estimated 3 million people die each year due to air pollution. ${ }^{1}$ While

U.S. pollution problems are far less severe than those in developing countries, half of

America's residents live in areas that violate health-based air quality standards despite

over thirty years of effort to clean up the air. ${ }^{2,3}$ Air quality planning is a "wicked"

problem that has no definitive definition, no obviously correct solutions, contested and

competing values, and considerable uncertainty. ${ }^{4}$ It is also a trans-scientific problem

\footnotetext{
${ }^{1}$ The World Health Organization estimated that between 1.4 and 6 million people die each year from air pollution (WHO 2000). The estimated median rate of 3 million deaths represents five percent of annual worldwide mortality.

${ }^{2}$ The Environmental Protection Agency estimated that 129 million people live in areas that violated National Ambient Air Quality Standards (EPA 2002), nearly half the 280 million people in the U.S. (Census 2000). Tropospheric ozone is particularly troublesome. According to monitoring data gathered in 2000, approximately 52 million people lived in 30 metropolitan statistical areas where the highest second daily maximum concentration violated the ozone NAAQS threshold of $0.12 \mathrm{ppm}$ averaged over one hour (EPA 2002). The federal ozone NAAQS adopted by EPA in 1997 is a new challenge for air quality planning. The new standard's threshold concentration of $0.08 \mathrm{ppm}$ averaged over eight hours was exceeded by the highest fourth daily maximum measured in over 100 areas with a population of 119 million people (EPA 2002). Note, however, that EPA's use of metropolitan population together with ambient air quality standards violation data may be misleading since a large portion of the population may not be exposed to the high levels of air pollution detected at monitoring stations.

${ }^{3}$ Federal legislation commenced with the Air Pollution Control Act in 1955 (1955), followed by the first Clean Air Act in 1963 (1963). The first substantive legislation was passed in 1970 (1970). California efforts started in 1947, when the state legislature established an Air Pollution Control District in Los Angeles (Hyink et al. 1959).

${ }^{4}$ Rittel and Webber (1973) describe "wicked” planning problems in several dimensions:

1. No definitive formulation of the problem

2. Solution has no stopping rule

3. Solutions are not true/false, they are good/bad

4. There is no immediate or ultimate test of a solution

5. Every solution is a "one-shot operation", cannot learn by trial-and-error

6. No clear set of potential solutions, nor any clear set of permissible actions

7. Every problem is essentially unique

8. Every problem can be considered a symptom of another problem
} 
because it can be directed to scientists for solution but not solved by them (Weinberg 1972). With no where else to turn, however, lawmakers placed great faith in science when adopting the Federal Clean Air Act (FCAA).

Research and planning efforts have improved understanding of the sources of air pollution, technologies for controlling it, and planning tools for assessing the issues and options. We can describe complex combustion kinetics and chemistry, atmospheric processing, and air/surface interactions. We measure routinely ambient conditions at hundreds of monitoring stations. Billions of dollars are now invested in scrubbers, catalysts, cleaners and conservation to reduce emissions or avoid them altogether. One essential planning tool is a photochemical air quality simulation model (PAQSM) ${ }^{5}$. In many respects, a PAQSM represents the pinnacle of scientific achievement. Using measurements as inputs to mathematical formulas, it simulates on a computer the complex chemical and physical processes, and social behaviors that lead to unhealthy air. The PAQSM also projects hypothetical future conditions. If this latter task shows that, once implemented, proposed emissions controls will yield acceptably clean air, it satisfies the "attainment demonstration" required of air quality management plans and thus provides justification for costly emissions controls.

Great scientific advances are in contrast to planning that, by 1990, had "largely failed” (NRC 1991). Since 1990, progress in attaining the 1-hour ozone standard in many regions "has been less than expected" (NARSTO 2000a). Though emissions controls are

9. Description of the problem is incomplete in many ways.

10. Choice of description determines the nature of the problem's resolution.

11. The planner has no right to be wrong.

${ }^{5}$ For a diagram and detailed description of a photochemical air quality simulation model (PAQSM) refer to Chapter III: PAQSM and Their Uncertainties. For the "attainment demonstration" requirements, refer to 
ubiquitous, ozone air quality conditions still regularly violate standards. Figure I-1 shows limited progress in reducing the number of days violating the federal 1-hour ozone standard in the San Joaquin Valley Air Basin, which is the case study region for this research. ${ }^{6}$ The region averaged over 30 violations per year from 1999 through 2002 despite considerable emissions reductions based on a plan adopted in 1994 that used modeling to predict attainment of the ozone standard (i.e., averaging 1 violation per year) by 1999 (see dotted line in Figure I-1). Furthermore, the supporting PAQSM application was the result of an \$18 million multi-agency, multi-stakeholder, ten-year research effort that produced a state-of-the-science model and a robust observational database (SJVAQS 1996).

Figure I-1: Ozone Trends in the SJV Air Basin

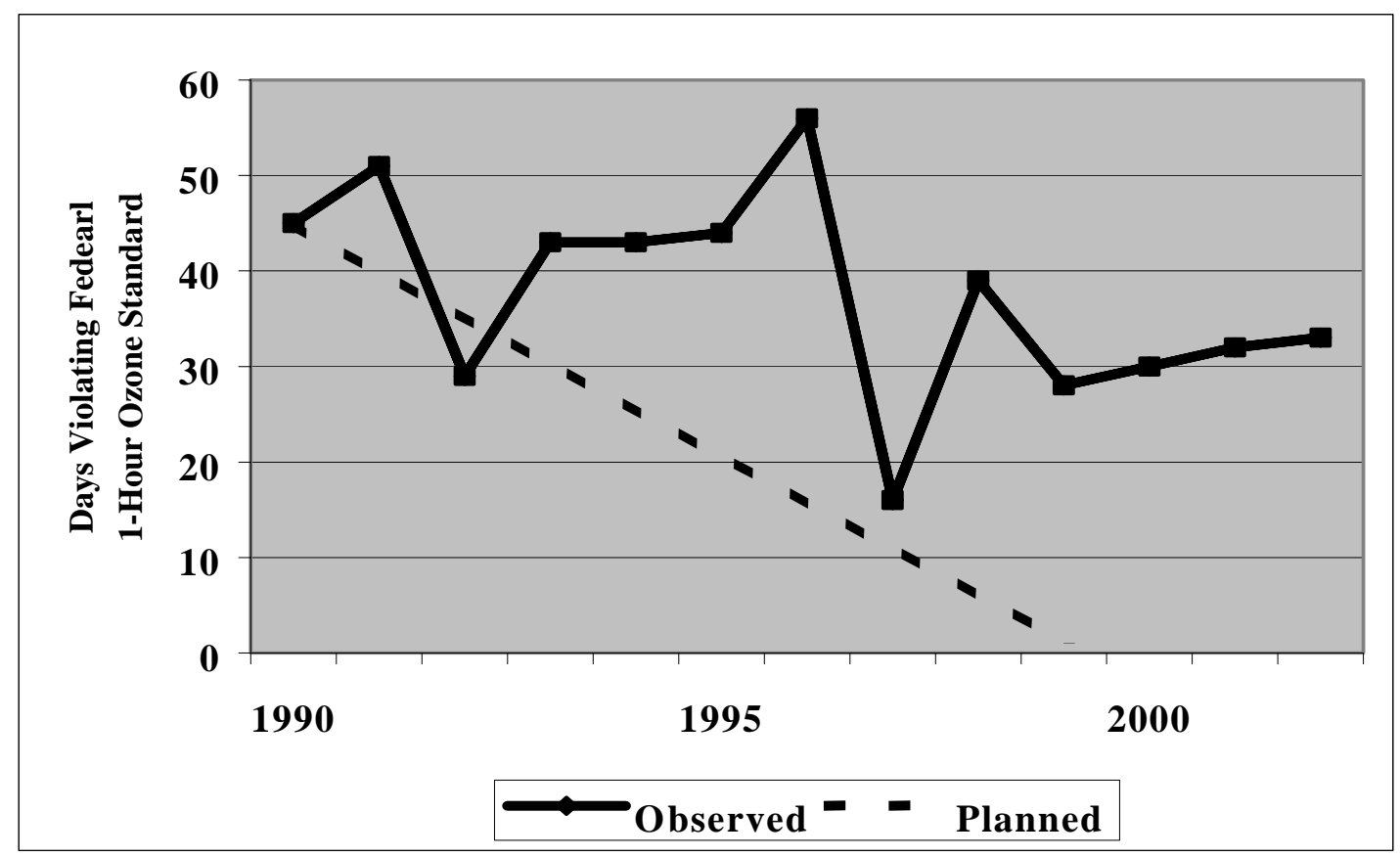

Modeling Requirements for Ozone Attainment Demonstration Plans in Appendix B.

${ }^{6}$ Though the downward trend in Figure I-1 is slight, control efforts have averted potentially worsening 
Continuing violations of ambient air quality standards may be due to many factors, including unidentified emissions sources, insufficient planned controls, incomplete control implementation, or controls that are less effective than anticipated. Regardless, modeling results presented in plans repeatedly predict that goals will be met only to be proven wrong subsequently. That model results are uncertain is neither surprising nor new (Tesche 1988; NRC 1991; Russell 1997; NARSTO 2000b). ${ }^{7}$ What is surprising is that considerable engineering experience and well-developed decision and statistical theory (for examples, see Stokey and Zeckhauser 1978; Finkel 1990; Morgan and Henrion 1990) have not been utilized to assess and manage the risks associated with air quality plans. ${ }^{8}$ The uncertainties in modeling are not dealt with explicitly in the decisionmaking process despite the well-documented advantages of doing so. This research explores why not by examining how scientific information is used in an actual planning process and how additional information about uncertainty might be generated and used.

\section{B. Stories about the Problem}

There are several ways to describe air quality issues in Central California. On April 23, 2003, Mr. David Crow, Air Pollution Control Officer of the San Joaquin Valley Air Pollution Control District, commenced Operation Clean Air, a new initiative to address air quality in the San Joaquin Valley (SJV), by saying there are two ways to talk about the problem. The first starts with health effects, such as rising childhood asthma rates in

\footnotetext{
conditions due to emissions associated with large population growth in the region.

${ }^{7}$ In this context, uncertainty is more than inaccurate modeling results. It includes the use of modeling results for decision-making. Uncertainties associated with modeling and their sources are discussed in Chapter II - PAQSM and Their Uncertainties.

${ }^{8}$ The federal Clean Air Act provisions call for contingency plans containing additional emissions control measures. The provisions do not address explicitly uncertainties associated with the modeled "attainment demonstration" and have thus far contained controls insufficient to meet air quality standards.
} 
the SJV that are already twice the national average. In this view, air pollution must be reduced to stop the high toll on public health.

A second view of the problem is economic, where poor environmental quality makes it difficult to attract new business, yet emissions control costs also impede economic growth. In a region with an already depressed economy, this story often juxtaposes the need for revenues, agricultural and energy products, and jobs with costs that include air pollutant emissions, their effects, and mitigation efforts. The examination of costs and benefits is based on an implied pursuit of efficiency. That is, actions hinge on a desire to have benefits greater than costs, even when both sides of the ledger include incommensurate, non-quantifiable, non-market goods, services, impacts and losses. Inevitably, equity must be considered, too, by digging deeper than net revenues to identify who is winning and losing.

Focusing on law, public administration, and planning leads to explanations focused on agencies struggling with incompatible value systems (industrialists versus environmentalists), possibly conflicting goals (economic vitality and environmental quality), overly prescriptive laws (unreasonable deadlines), insufficient resources (lack of staff), and scientific uncertainty (inadequate understanding and tools). Legal scholars talk of the dangers of symbolic legislation (Dwyer 1990), adversarial legalism (Kagan 1991), and the necessity of improved legislative and judicial approaches. In these views, the litigious nature of planning is, depending on your perspective, either impeding progress or the only thing driving it. Fear of litigation leads to incremental decisionmaking in the face of uncertainty (Greenberger et al. 1976), when instead drastic measures are necessary. 
Another way to approach air quality issues is to examine the science: what we do and do not know about the atmosphere and the complex interactions between human activities, pollution, and health. This story focuses on progress made to date in the research realm and the incomplete translation of that knowledge to decision-making.

\section{The Case}

In this research, those involved in the process tell of their experiences with air quality planning in Central California during the 1990’s. Their opinions are recapitulated into meta-stories, or narratives. The narratives have beginnings, middles and ends, and incorporate facts selectively (Roe 1994). In this case, there are disagreements about the facts. The stories do not all begin at the same place because their tellers have different definitions of the problem and, thus, incorporate the facts differentially. Consequently, it is no surprise that the narratives lead to conflicting conclusions about, first, what is known about the problem(s) and, second, what should be done about it (them).

The narratives are not considered in isolation. Before getting to them, considerable detail about Central California is provided about regulatory, socioeconomic, and environmental conditions in the region. With this understanding, narratives are identified as part of the description and critique of the air quality planning process. Particular attention is focused on the science and associated uncertainties. Uncertainty is one crux point by which narratives differentiate; it is where controversy begins because knowledge is not sufficiently definitive to coalesce agreement amongst those with different values, world-views, risks, responsibilities and objectives. 


\section{Control Strategy Options}

Ozone $\left(\mathrm{O}_{3}\right)$ forms in the atmosphere through complex chemical reactions involving oxides of nitrogen $\left(\mathrm{NO}_{\mathrm{x}}\right)$ and volatile organic compounds (VOCs) in the presence of heat and sunlight (Seinfeld and Pandis 1998). Ozone forms from nonlinear processes that can be counterintuitive. Either of the two precursor pollutants might be the limiting reagent for ozone formation, but it may vary with time and place at sub-urban scales. For example, depending on conditions, reducing emissions of nitrogen oxides can increase ozone or reducing VOCs emissions might have little affect on ozone levels. The "control strategy" is a choice to reduce emissions of both precursor pollutants or one preferentially, as well as when, where and how much to reduce.

A tool used commonly for studying how the ozone concentration depends on precursor emissions is a PAQSM. An isopleth diagram shows the relationship between precursor emissions and peak ozone. Isopleths are produced by executing a PAQSM many times, each time with a different assumption about the amount of precursor emissions $\left(\mathrm{NO}_{\mathrm{x}}\right.$ emissions are on the $\mathrm{y}$-axis, VOCs emissions on the $\mathrm{x}$-axis). Each modeling run provides a point; many points eventually create a surface. Thus, the isopleth diagram is a visual depiction of the sensitivity of peak ozone to changes in precursor emissions. Typically, as was the case in Central California, the isopleth is derived from the simulation of one ozone event representing one set of meteorological conditions.

Figure I-2 is an isopleth from the most recent ozone attainment demonstration plan for the San Francisco Bay Area (Bay Area) (BAAQMD 2001a). The local planning agency, Bay Area Air Quality Management District (BAAQMD), used it to argue that the 
region is currently at point $\mathrm{A}$, in the upper right-hand corner, and needs to get to point $\mathrm{B}$ to meet the federal 1-hour ozone standard. As emissions decline, ozone is presumed to decline from point A toward the origin. If one believes this graphic, it can be used to determine what controls of $\mathrm{NO}_{\mathrm{x}}$ and VOCs are necessary to meet the standard.

This isopleth is a very uncertain picture of ozone sensitivity in the Bay Area. ${ }^{9}$ However, other research corroborates it to suggest that $\mathrm{NO}_{\mathrm{x}}$ controls are not only ineffective, but might actually worsen air quality locally and make it tougher to meet the standard (Martien et al. 1992; Martien 1993; Fairley 1996; Kaduwela 1996). Note the shape of the contours. If $\mathrm{NO}_{\mathrm{x}}$ emissions are not reduced (i.e., they remain at 752 tons per day), VOC emissions need only be reduced to approximately 570 tons per day (tpd) to reach the contour line representing $124 \mathrm{ppb}$ of ozone, the federal standard after rounding down. Because $\mathrm{NO}_{\mathrm{x}}$ controls are planned (see dashed arrow in Figure I-2), VOCs must be reduced to 524 tpd, an extra 36 tons.

\footnotetext{
${ }^{9}$ Isopleths have uncertainties associated with the estimates of $\mathrm{NO}_{\mathrm{x}}$ and VOC emissions, the model itself, the ozone forming potential of different VOCs, and the types of meteorological conditions and locations represented. Nonetheless, it is a heuristic device used commonly for studying ozone.
} 
Figure I-2: Bay Area Isopleth for Livermore (BAAQMD 2001a)

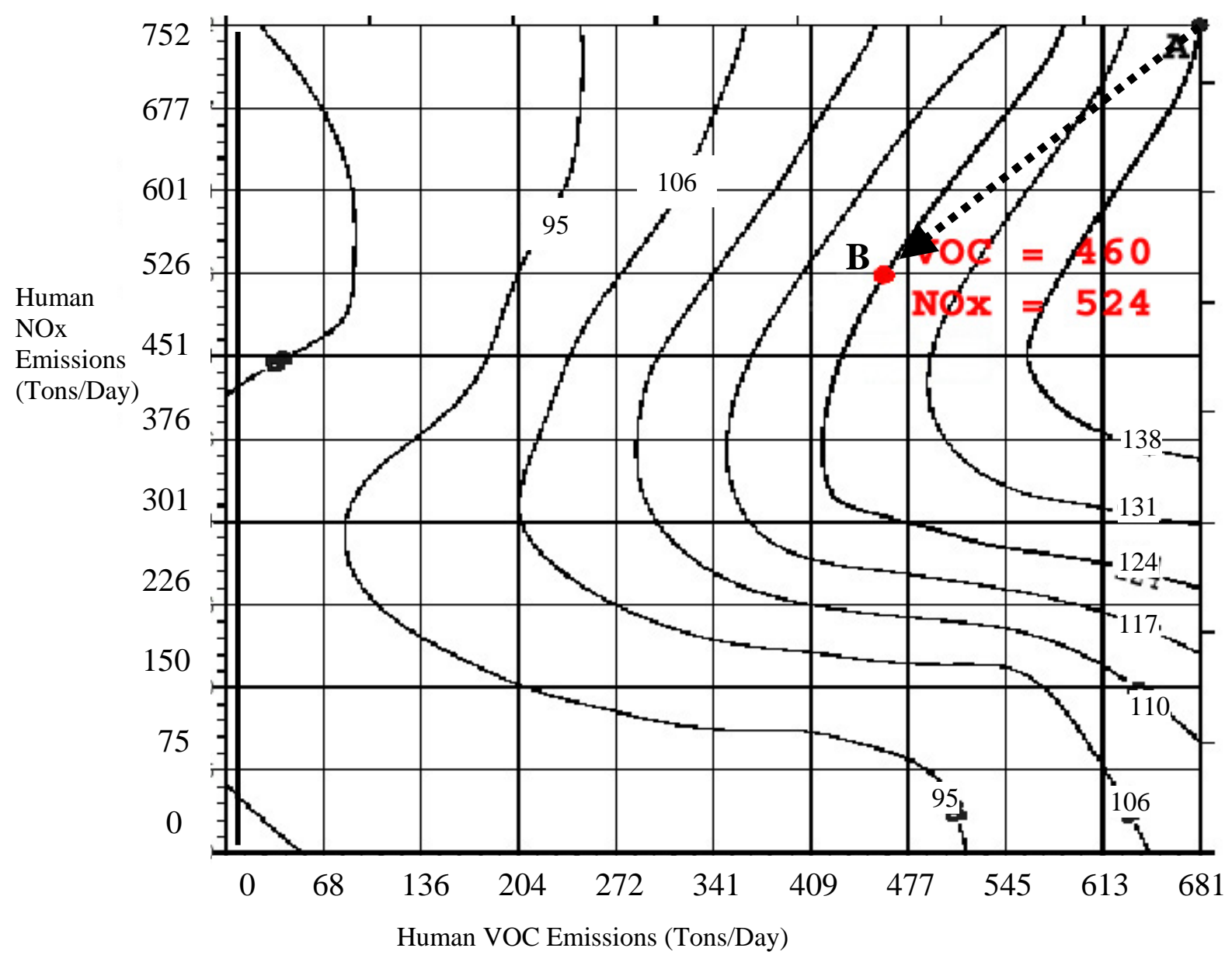

The Bay Area isopleth depicts visually the different arguments about the preferred emissions control strategy for the region. The regulated community understandably wants to install as few costly controls as possible. They argue that, according to the above isopleth and other corroborative evidence, the control strategy should focus on reducing only VOCs (Ziman 2000).

Environmental advocates do not rely on the isopleth (Angel 2001; Ramo 2001). Instead, they see people suffering as the most relevant evidence and thus argue that emissions should be reduced to harmless levels. Furthermore, they note that ozone 
precursor pollutants (i.e., $\mathrm{NO}_{\mathrm{x}}$ and VOCs) are themselves dangerous pollutants and contribute to other air quality concerns, such as ambient particulate matter, so emissions controls need not be justified by ozone standards alone. Their call for reductions might be based on their understanding of controls implemented in other regions (for example, see Sher 1998), or their belief that planning agencies' definition of “feasible” controls has been biased by politically and economically powerful industrial interests. ${ }^{10}$

The public agents at the BAAQMD are caught in between, realizing that they can require only emissions reductions justified by the "best available science." ${ }^{11}$ In deciding apparently inevitable lawsuits, judges rule on procedural, not scientific grounds, assuring only that the agency's decision is neither arbitrary nor capricious. ${ }^{12}$ Basing decisions on modeling results - the best available science - facilitates favorable judicial rulings.

Regulators also want to feel good about their work by cleaning up the air. ${ }^{13}$ Their authority only goes so far, however. ${ }^{14}$ For example, significant emissions in the S.F. Bay Area are produced by motor vehicles (BAAQMD 2001a), but vehicle emissions limits are set at the state and federal levels, not locally (FCAA 1990, Title II).

Another influential opinion rests with the California Air Resources Board (CARB). As the state agency with primacy over California air quality, CARB has approval authority over plans submitted by local planning agencies, such as the BAAQMD and San Joaquin Valley Unified Air Pollution Control District (SJVUAPCD), as well as a

\footnotetext{
${ }^{10}$ For example, see the Greenaction website at www.greenaction.org, last visited May 15, 2003.

${ }^{11}$ For example, emissions control technologies are based on the "Best Available Control Technologies" (FCAA 1990). Also, see quotes of Ms. Lynn Terry, CARB, and Mr. Robin DeMandel, BAAQMD, in Chapter IX discussion of Model Performance Evaluation.

${ }^{12}$ See footnote in Objective Science discussion in Chapter II.

${ }^{13}$ For example, see quote of Ms. Lynn Terry in Winning the Game discussion of Chapter IX who
} 
large technical support division that identifies control measures, oversees regional studies, and provides modeling assistance to air districts. ${ }^{15}$ The policy forwarded by CARB calls for implementation of all feasible emissions controls of both $\mathrm{NO}_{\mathrm{x}}$ and VOCs. CARB sites several air quality concerns in addition to ozone, notably particulate matter and visibility degradation, and chooses what it considers to be a precautionary approach to mitigate scientific uncertainty. ${ }^{16}$

According to the regulated community, CARB's approach is the worst possible policy because it is inefficient and expensive path toward reducing ozone, per the nonlinear relationships shown in Figure I-2. ${ }^{17}$ Furthermore, they argue that, in the case of the Bay Area, $\mathrm{NO}_{\mathrm{x}}$ controls may increase peak ozone concentrations locally. Research by the BAAQMD and CARB supports this argument (Martien et al. 1992; Martien and Umeda 1993; Fairley 1996; Kaduwela 1996; Ranzieri 1996). It can be understood by referring again to Figure I-2. If only $\mathrm{NO}_{\mathrm{x}}$ emissions are reduced, ozone will increase because conditions will move from point A, the current ozone condition, in a perpendicular direction toward the $\mathrm{x}$-axis and up to a higher ozone concentration.

Industry laments that CARB ignores scientific results whenever the dual control strategy is not supported, thereby failing to fulfill its fiduciary responsibility of finding

\footnotetext{
highlights the public health improvements from emissions reductions.

${ }^{14}$ See Jurisdiction and Decision-making Authority discussion in Chapter IX.

${ }^{15}$ For description of the responsibilities of CARB, EPA and local air quality management agencies in California refer to Air Quality Management Agencies in Appendix B.

${ }^{16}$ See quotes of Ms. Lynn Terry and Mr. Michael Scheible, CARB, in Chapter IX discussion of Problem Definition.

${ }^{17}$ See quotes of Mr. Michael Wang, Western States Petroleum Association, and Dr. Steven Ziman, Chevron-Texaco, in Chapter IX discussion of Models and Uncertainty.
} 
the most efficient control strategy. Instead, says industry, CARB uses science and uncertainty to justify their predetermined policies. ${ }^{18}$

The above arguments, exemplified by quotes referenced in footnotes, reveal how each group uses scientific evidence to support their opinions. Industry highlights evidence, such as the isopleth shown in Figure I-2, that suggest a cheaper path to acceptably clean air. Their concerns are relevant and justifiable, but are not necessarily reason to forgo controls until answered. Similarly, CARB claims the models are too uncertain for use in defining precise control strategies and notes air quality concerns beyond ozone. These competing arguments, based all the while on what "the science says", raises the question of how science is actually used for decision-making.

\section{Opinions About Models}

The people interviewed for this work do not believe that modeling determines emissions reduction plans. That is, the modeling does not delimit what emissions need to be reduced. This is where opinions start to differ. Some believe decisions are made prior to modeling studies. Twenty-five years ago, policy scientists discovered modeling used to delay, avoid, or justify a priori decisions (Brewer, 1973b) and that modeling results are considered in the policy context only when politically salient (Greenberger et al., 1976).

Planners interviewed for this research say that time and resource constraints reduce the role of science. Research is cut short by lack of funds and regulatory deadlines for plan submission. Thus, the process goes on, and plans are submitted that are actually

\footnotetext{
${ }^{18}$ See quotes of Dr. Eugene Leong, ABAG, and Dr. Steven Ziman, in Chapter IX discussion titled Winning the Game.
} 
interim reports based upon incomplete understanding (for example, see Howekamp et al. 1994).

In addition to time, the availability of "feasible" controls was cited by many of those interviewed as obviating the science. The definition of feasible, however, is the result of a political negotiation, not purely an objective assessment of technical capability and cost-effectiveness. All interviewed for this work agreed that air quality planning is an inherently political process. If the modeling indicates the need for more reductions than “feasible”, then improving the model will only be useful if it indicates fewer controls are needed to meet air quality goals. There exists a common, though erroneous, assumption amongst those interviewed that that consideration of modeling uncertainties will indicate the need for more emissions controls. The Bay Area isopleth (Figure I-2) suggests the opposite may be true.

The modeled “attainment demonstration” used in ozone plans would be less convincing if uncertainties were made explicit. The language of the FCAA does not allow for gray areas; modeling “demonstrations” either show future attainment or they do not. $^{19}$ Legally, the Environmental Protection Agency (EPA) cannot accept a plan indicating goals will probably be met, even though this disclaimer is understood. Instead, EPA approves only plans that include a modeling simulation showing that planned controls will be sufficient to prevent unacceptably high ozone concentrations if in the future meteorological conditions that led to past violations are experienced again (EPA $1996 \mathrm{~b}) .^{20}$

\footnotetext{
${ }^{19}$ See language of the CAA in Chapter II section discussing Objective Science.

${ }^{20}$ See discussion of Modeling Requirements of Ozone Attainment Demonstration Plans in Appendix B.
} 
This work examines decision-makers’ interest in, understanding of, and efforts to manage and communicate uncertainty. At each level of decision-making, from the modeler, who gives results to the planner, to the planner, who makes recommendations to their Board, to the elite policy-maker seeking plan approval from, first, CARB and, then, EPA, there is less and less communication about the science and associated uncertainties. Modelers accept responsibility for reducing uncertainties, while the planners want to be given simply the "best available" results. ${ }^{21}$ Experienced planners believe they internalize the uncertainties when making decisions. ${ }^{22}$ Furthermore, they argue that members of their agencies’ boards, as well as the general public, want simplified information accompanying decisions that they can feel good about, rather than an exposition of the uncertainties. This reality would not be of concern if the plans were successful, but they are not. Might the technical staffs at CARB and air districts be asked to do more in facilitating risk assessment and management? What more can be done? Why have executive-level decision-makers not sought more information about the uncertainties?

The question facing those developing a plan to meet the federal ozone NAAQS is: How much must current $\mathrm{NO}_{x}$ and VOC emissions be reduced to meet the federal ozone standard (by a specified deadline)? To consider the uncertainty, a second question is relevant when plans rely on a modeled “attainment demonstration” to justify planned emissions reductions: What is the probability that the federal ozone standard will be met when the model says so? This probabilistic assessment is a way to manage uncertainty

\footnotetext{
${ }^{21}$ For example, see Mr. Robin DeMandel quote in Model Performance Evaluation discussion of Chapter $I X$.

${ }^{22}$ For example, see Mr. Michael Scheible quote in Model Performance Evaluation discussion of Chapter $I X$.
} 
(Stokey and Zeckhauser 1978; Morgan and Henrion 1990), yet it has not been adopted for use by air quality managers.

Many research efforts have produced information about PAQSM uncertainties (for a recent review of those efforts, refer to Fine et al. 2003). The scientific literature contains many scholarly suggestions for corroborating and alternative analyses to provide the basis for assessing and, if necessary, reorienting emissions control strategies (for example, see Demerjian et al. 1995). Many of the suggestions rely on careful analyses of models' uncertainties. The research presented here explores why these suggestions are not used for planning and evaluates the potential for implementing them in the future.

\section{Research Goals}

This dissertation has three goals:

- Descriptive To explain how models and uncertainties are used in air quality planning, while identifying useful uncertainty information and assessing capabilities to produce it

- Assimilative To draw from policy science, decision science, science and technology studies, planning and modeling theory to critique air quality planning and to suggest improvements reliant on the integration of model uncertainty information

- Prescriptive To provide recommendations planners can use to implement suggested improvements

In pursuit of these goals, the following questions are explored:

- How does air quality planning work in practice?

- What are the dominant stories and disagreements associated with air quality planning in Central California?

- How are models and uncertainty used in current air quality planning practice?

- What are the potential uses of explicating model uncertainty information? 
- How might potentially useful uncertainty information be produced and utilized?

- What technical and political impediments prohibit the integration of uncertainty information?

\section{Methods and Research Products}

This research is based on a case study of air quality planning in Central California during the 1990s. Primary data consist of documentation (e.g., agency reports, e-mails, modeling output) and thirty interviews that I conducted with people familiar with the process. The work focuses on the San Joaquin Valley because it has among the Nation's worst air quality and a well-funded, state-of-the-science, ongoing research effort. The San Francisco Bay Area is an integral part of the story, too, as it is implicated as an upwind source of pollution to the SJV.

I describe the process using diagrammed networks of interactions and policy narratives. Narratives are delineated by gathering and comparing stories about the process, assessing the facts, and identifying controversies. In addition, I identify points of common agreement, and assess the state of scientific understanding and capability.

I then critique the process using theory from the disciplines of air quality engineering, decision science, policy science, consensus-based and communicative planning, modeling, economics, and science and technology studies.

Finally, I juxtapose findings about the case study with a review of past efforts and current capabilities to produce model uncertainty information. My recommendations consider capabilities regarding PAQSM uncertainty assessment and then address the social aspects of science-led planning. 


\section{PLANNING, MODELING AND UNCERTAINTY}

\section{A. Introduction}

This chapter surveys the literature about using science to inform decision-making for environmental planning. The intent is to provide context for this research by exploring several theoretical literatures concurrently. I begin with three logical positivist claims:

1. Objective information is needed for environmental decision-making.

2. Scientific research provides information per the needs of decision-makers.

3. Modeling research is uniquely capable of producing some needed information.

Building upon these claims and the policy sciences and social studies of science and technology literatures, I conclude:

4. Modeling applications are not purely objective, due to limitations of uncertainty, opacity and subjectivity.

5. Air pollution, like many other environmental problems, cannot be managed solely through scientific research because it involves significant, inequitably-shared risks and burdens, competing values, and contested science.

Finally, observing that lawmakers placed great faith in objective and informed decisions, I argued for two responses to the inability of science to provide reliable information without controversy:

A. Modeling and other research results should be presented and used in ways that address and mitigate their limitations.

B. Planning processes should be consensus-based and should employ a set of principles for generating, communicating and considering information.

Identified in this chapter are the decision-maker's tasks, information needs, and methods and tools, such as models, available to provide that information. The limits of models, and other challenges facing planners are discussed. Questions explored are:

- Why model? Model for whom? 
- Are decisions based on models?

- What is the relationship between science and policy?

To motivate this research, this chapter leaves more questions than answers.

\section{B. Decisions To Be Made}

Rittel and Webber (1973) describe several classical, simultaneous planning duties:

1. identifying problems

2. forecasting uncontrollable contextual changes

3. inventing alternative and plausible action sets and their consequences

4. evaluating alternatively forecasted outcomes

5. statistically monitoring conditions thought to be germane

6. feeding back information to the simulation and decision channels to correct errors

In the case of air quality planning, problem assessment involves monitoring and evaluating air quality with respect to legislated and regulated goals, and identifying the magnitudes, sources and methods to reduce pollutant emissions. When air quality goals are not being met, the primary task becomes determining what emissions reductions are necessary to meet goals at some future date. Proposed or anticipated new sources of emissions due to growth must also be considered.

Drawing from Demerjian et al. (1995), questions pertaining to pursuit of National Ambient Air Quality Standards (NAAQS) for ozone are:

- Conditions: What are current air quality conditions? What are anticipated air quality conditions under a 'business as usual” scenario?

- Quantity: How much must current $\mathrm{NO}_{\mathrm{x}}$ and VOC emissions be reduced to meet the ozone NAAQS (by a specified deadline)? ${ }^{23}$

\footnotetext{
${ }^{23}$ Although the focus here is on ozone standards, other air quality standards are also relevant because air pollutants may be interdependent.
} 
- Local culpability: What sources within the airshed must reduce their emissions?

- Transported culpability: What upwind sources contribute to local ozone concentrations, and how much do local emissions cause downwind ozone formation?

- Pollutant type: Which of their emissions must be reduced (i.e., $\mathrm{NO}_{\mathrm{x}}$, VOCs, or both)?

- Temporal and spatial specification: When and where must emissions be reduced?

- Projection: What changes in current or anticipated emissions are necessary to achieve or maintain air quality goals in the future?

- Sensitivity: What are the relationships between emissions, emissions reductions, and air quality conditions?

These eight questions create the need for information provided by researchers.

\section{Informing Decisions}

Methods available to the researcher include (Demerjian et al. 1995):

- Observations of air quality conditions, meteorology or emissions at their sources

- Estimation of emissions using observations and engineering principles

- Analysis of past and anticipated trends about conditions or activities, including statistical modeling

- Laboratory studies

- Simulation modeling

For air quality planning, observations and emissions estimates provide partial information to address the quantity, culpability, conditions and projections questions. Trends analysis informs the conditions and projections assessments. Laboratory experiments contribute to assessment of culpability, emissions estimation, sensitivities 
and choices about the pollutants to control. However, only simulation modeling can inform fully the set of questions in an integrated manner (Brewer 1973b; Greenberger et al. 1976). ${ }^{24}$ Results from lab experiments must be extrapolated to real-world scales, whereas simulation may represent an observed event at an airshed (or larger) spatial scale. Most important, models can prognosticate by simulating alternative futures.

The information advantages of models contribute to their popularity for environmental planning, in general, and air quality planning, in particular. Another reason they are used is that they represent an objective means of informed decisionmaking (Aronson 1984, Ozawa 1996).

\section{Objective Science}

Models provide researchers the means for making "cognitive claims” about the natural world (Aronson 1984). The preeminent role of models derives from the ideals of progressive governance and logical positivist empiricism, whereby data are “incontrovertible and unchanging” (Ozawa 1996). These data are assumed generated and analyzed by objective scientists to produce value-neutral information for use by disinterested policy-makers. Science is viewed as politically neutral (Ozawa 1991; Ozawa 1996). Rationalist and positivist presumptions underlie laws and regulations that treat modeling studies as objective, and the output from these studies as sufficiently reliable to prescribe without controversy actions necessary to achieve air quality goals. For example, the courts give public agents discretion to use modeling studies as evidence

\footnotetext{
${ }^{24}$ Additional uses of models are discussed in the next chapter.
} 
that their decisions are neither arbitrary nor capricious and trust the public to call into question the modeling as necessary. ${ }^{25}$

There is no mention of the use of modeling in early clean air legislation. The 1990 FCAA Amendments, however, reference the use of air quality models over 30 times. $^{26}$ The FCAA creates a planning processes that relies upon PAQSMs to "demonstrate" that ozone NAAQS will be attained ${ }^{27}$. Demerjian et. al. (1995) diagram the process as shown in Figure II-1. The State Implementation Plan (SIP) codifies the demonstration.

The process conceived in laws and regulatory guidance assumes an idealized role for technical information as depicted in Figure II-2. For example, the CAA requires that NAAQS be set to protect human health with an adequate margin of safety, allowing no

${ }^{25}$ In Chevron U.S.A. vs. Natural Resources Defense Council (NRDC), NRDC challenged EPA's approach for applying emissions controls to meet the requirements of the Clean Air Act Amendments of 1977. The U.S. Supreme Court supported EPA, stating, "the EPA should have broad discretion in implementing the policies of the 1977 Amendments [of the Clean Air Act]" (467 U.S. 837). This opinion confirmed that agencies are given "broad discretion" to interpret environmental statutes. The opinion further states, If Congress has explicitly left a gap for the agency to fill, there is an express delegation of authority to the agency to elucidate a specific provision of the statute by regulation. Such legislative regulations are given controlling weight unless they are arbitrary, capricious, or manifestly contrary to the statute.

Treating modeling as the codification of scientific expertise, in Sierra Club vs. Costle, EPA Administrator, the U.S. Supreme Court's majority opinion stated,

We still cannot agree with the Sierra Club that it was improper for EPA to employ an econometric model....Computer modeling is a useful and often essential tool for performing the Herculean labors Congress imposed on EPA in the Clean Air Act.....Given the complexity and magnitude of the analyses EPA must perform...computer modeling, for all of its flaws, is invaluable...We are in fact reassured by EPA's own consciousness of the limits of its model, and its invitation and response to public comment on all aspects of the model. The safety valves in the use of such sophisticated methodology are the requirement of public exposure.

${ }^{26}$ For an example, refer to the language presented in Appendix B discussion, Modeling Requirements for Ozone Attainment Demonstration Plans.

${ }^{27}$ If the ozone NAAQS are not met within an air district, the local management agency must simulate the air quality using a photochemical computer model, and then use the model to "demonstrate" that the NAAQS will be met once emissions controls are implemented. 
provision for economic or technical considerations (FCAA 1990). Politics is presumed to play no role in any of these decision-making processes. ${ }^{28}$

Figure II-1: Ozone NAAQS Planning Process (Demerjian et al. 1995)

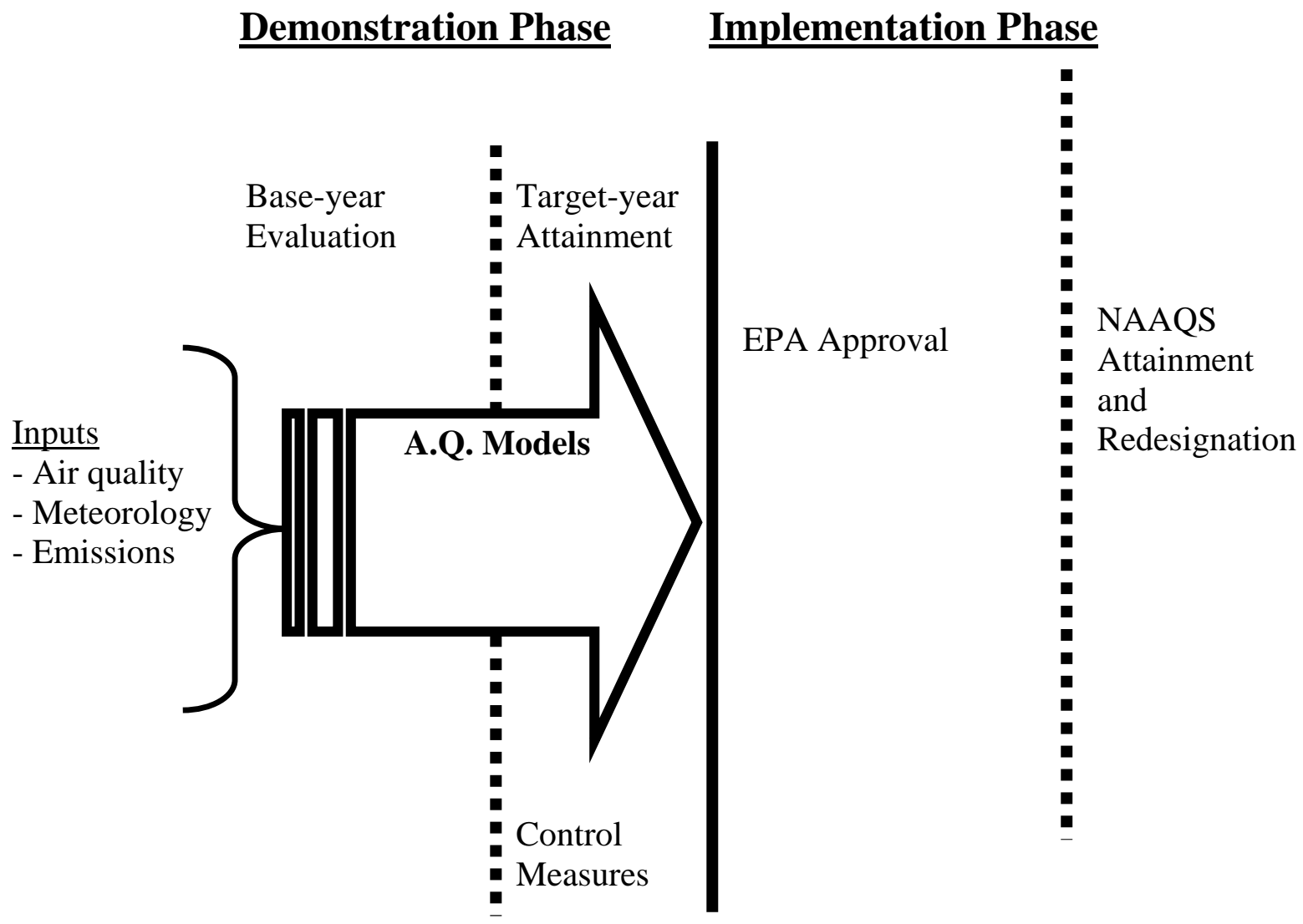

${ }^{28}$ Politics refers to the definition of organizational politics provided by Pfeffer (Power in Organizations, 1981) and cited in Fischer (1990) as "the activity undertaken by individuals or groups 'within organizations to acquire, develop and use power...to obtain...preferred outcomes in a situation in which there is uncertainty or dissensus about choice’”. 
Figure II-2: Idealized Role of Models in NAAQS Planning

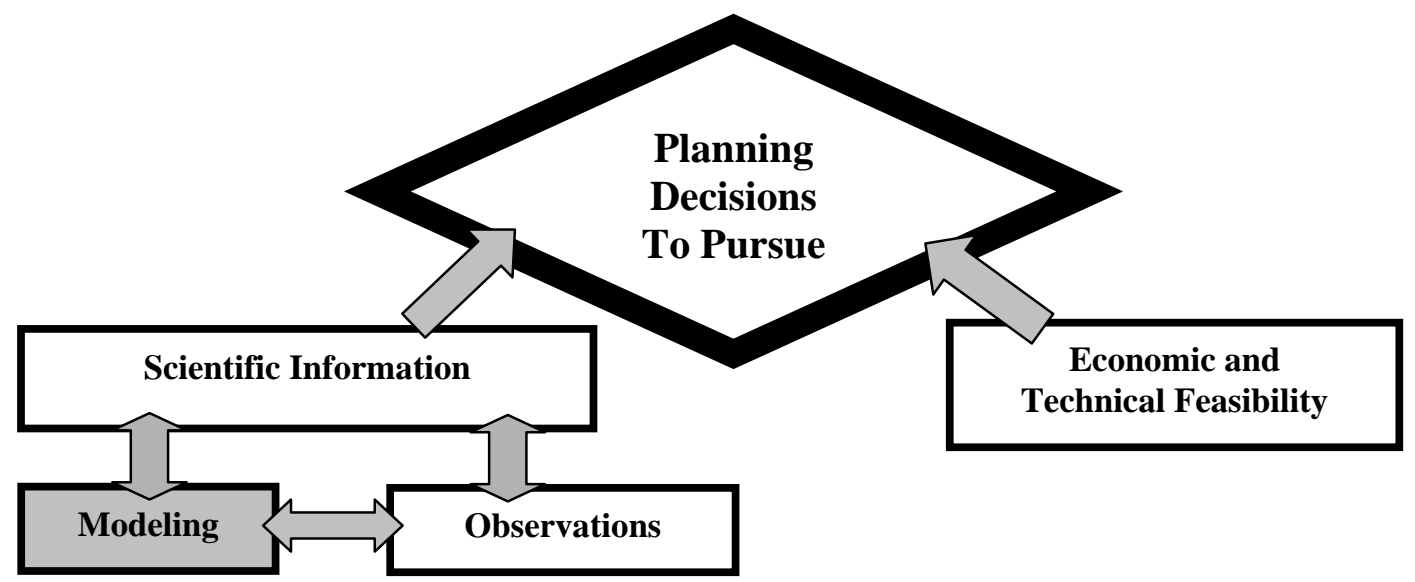

\section{E. Air Quality Modeling Limitations}

Are models capable of providing incontrovertible, unchanging, value-neutral information about the real world for use by air quality managers? The section argues that the answer is an emphatic no due to the myriad limitations of PAQSMs, which are discussed here in terms of uncertainty, opacity, and subjectivity.

\section{Uncertainty}

The past 30 years of State Implementation Plans provide ample evidence that modelbased air quality planning has not achieved air quality goals, since few plans produced air quality in compliance with ozone NAAQS. Even since the overhaul of the FCAA in 1990, progress in attaining standards has been slower than expected (NARSTO 2000a). By 1997, approximately three-quarters of areas not meeting ozone standards in 1990 had failed to enact plans that resulted in attainment (Roth et al. 1997).

The “discouraging and perplexing” (NRC 1991, page vii.) inability to reduce peak urban ozone concentrations inspired three federal studies (OTA 1989; NRC 1991; NARSTO 2000a) and a privately-sponsored review (API 1989). All reports noted 
significant modeling inaccuracies, and called for their evaluation and reduction.

Uncertainties are an unavoidable part of any modeling (Cleary 1976; Oreskes et al. 1994;

Morgan and Henrion 1990). The uncertainties associated with PAQSMs, and the broader decision-making context, are detailed in the next chapter. Here, the different reasons for uncertainty associated with air quality modeling are introduced.

\section{Prediction}

"It is the planners, more than perhaps anyone else, who would like nothing better than to have a machine to foretell the future,” wrote Schumacher (1973, page 225).

Demerjian et al. (1995) observed,

It is crucial to recognize that the history of air quality projections using PAQSMs is one of consistently overestimating the reductions in peak ozone concentrations that are to be realized in a specified period of time.

Ascher (1976) examines the accuracy of forecasts that are developed using models.

He offers general conclusions about the correlates with forecast accuracy:

1. Time horizon is the most important factor - forecast accuracy decreases with the distance of the forecast date

2a. Different results are obtained by different institutional sites of forecasting efforts, primarily in terms of systematic biases rather than absolute accuracy.

2b. Even so, the most common pattern is the appearance of the same bias in all forecast sources at a given point in time.

3. The choice of methodology in practice is not generally linked to difference in accuracy, in part because several methods are often used in combination, but also because the presumed advantages of sophisticated methodologies simply have not yet materialized.

In his last conclusion, Ascher finds that accuracy is not necessary improved with sophisticated modeling methods, including the complexity of the modeling system itself. 
He also points out the fundamental difficulty of predicting the future, regardless of the method employed or institutional biases. A primary reason air quality managers conduct modeling is to predict future conditions, by way of demonstrating that controls planned today will yield cleaner air in the future. Unfortunately, past efforts exemplify the uncertainty associated with the prediction task (for example, see Sarewitz et al. 2000) .

\section{Complexity}

Pool (1997) wrote "Complexity creates unpredictability.” Models have been made increasingly complex in a reductionist attempt to reduce uncertainty. ${ }^{29}$ Indeed, the physical and chemical process of interest are themselves very complex. Perhaps this is why after 30 years of increasing sophistication, air quality simulation model performance has not improved substantially (NARSTO 2000b). Demerjian et al. (1995) make the following observation about the complexity of PAQSM,

The potential for uncertainty increases after a certain point, rendering overall acceptance of the model more difficult. Some feel that the bottom of the complexity-uncertainty curve has already been passed, and the community is being confronted with evaluation requirements that are increasingly difficult to meet.

"Getting the sign right" continues to be a challenge. The right sign means that the correct precursor pollutant, $\mathrm{NO}_{\mathrm{x}}$ or VOC, is controlled to reduce ozone. For example, a recent uncertainty analysis by Bergin et. al. (1999) estimated that a $25 \%$ reduction in $\mathrm{NO}_{\mathrm{x}}$ emissions would reduce peak ozone by $2.3 \%$ plus or minus $9.7 \%$ in Claremont, California. The example reveals that controlling the wrong pollutant in the wrong

\footnotetext{
${ }^{29}$ The evolution of increasingly complex modeling systems, as well as their rise to prominence in air quality planning, is detailed in the next chapter. So too are modeling system uncertainties.
} 
situation may actually increase peak ozone ${ }^{30}$, a counterintuitive result that is characteristic of a complex system.

PAQSMs simulate ozone better today than they did in the past. More monitoring data, greater computing power, improved and expanded algorithms, and more efficient, accurate mathematical solution methods are used ${ }^{31}$. Emissions estimates are ever improving, in terms of both accuracy and inclusiveness. State-of-the-science PAQSM represent processes not included in the first and second generation models. The first generation of computer models were simple functions relating precursor emissions and ozone linearly (Gunther 1982). The second generation models were zero- and onedimensional formulations, such as the Empirical Kinetic Modeling Approach (EKMA) (Gunther 1982, Roth et al., 1993). They simulated the nonlinear ozone photochemistry, accounted for changes in pollutant emissions over time, and included chemical reaction mechanisms. Today's PAQSMs simulate ozone concentrations in the three dimensions of space and time. ${ }^{32}$ Nonetheless, no single "state-of-the-science” model contains formulation depicting the most modern scientific understanding, and all still embody many uncertainties (Russell 1997).

\section{Signal-to-Noise}

Why should we be concerned that models are uncertain? The real question is, given their uncertainty, are models good enough? The uncertainty associated with the modeling output (i.e., noise) is often equal to the desired change in air pollutant

\footnotetext{
${ }^{30}$ Refer to Chapter $X$ for description of nonlinearity ozone chemistry.

${ }^{31}$ Refer to Chapter III or NARSTO (2000b) for a discussion of recent PAQSM improvements.

${ }^{32}$ Refer to Chapter III for a detailed description of PAQSM formulation.
} 
concentrations (i.e., signal). ${ }^{33}$ This “signal-to-noise” concern makes it difficult to be confident that given actions will have the desired effect on air quality (NARSTO 2000b;

Fine et al. 2003).

Signal-to-noise concerns may be exacerbated by the new 8-hour ozone NAAQS. The absolute value of the standard (80 ppb) is lower than the 1-hour standard (120 ppb), so the signal of interest may be smaller. Yet, the uncertainty cannot be expected to decline commensurately. The application of PAQSM to 8-hour averaging times, combined with inclusion of more rural chemistry and more dispersed emissions sources will present new challenges that increase uncertainty.

\section{Model Verification and Validation}

PASQMs fail to satisfy two fundamental premises of positivism: verifiability and falsifiability. The input requirements of the modeling system make it what formal logicians refer to as an “open system”. It is therefore not possible to verify a model’s truthfulness, only to prove it wrong (Oreskes et al. 1994).

Greenberger et al. (1976) stated that the first rule of modeling was “to be used, but not to be believed.” Morgan and Henrion (1990, page 68) were more pessimistic,

Any model is unavoidably a simplification of reality. Any real-world system contains phenomena or behaviors that cannot be produced by even the most detailed model. Even if a model is a good approximation to a particular real-world system and usually gives accurate results, it can never be completely exact. Careful thought leads us to the following disturbing conclusion: Every model is definitely false. [Italics in original.]

Despite the theoretical limits of models’ ability to depict reality, they can be validated within a strict set of operating conditions. To validate air quality model performance,

\footnotetext{
${ }^{33}$ For example, where tropospheric ozone concentrations may exceed the one-hour standard of $120 \mathrm{ppb}$ by
} 
measures of fit between model predictions and observations (i.e., estimates of error and bias) are generated and compared with performance criteria set by EPA and CARB. ${ }^{34}$ However, it is not possible to conclude that a model validated under one set of conditions is still valid under very different conditions or in a new location. Nonetheless, partial or "local" validation is treated as sufficient evidence that the model is performing well enough to inform air quality plans (for example, see EPA 1996b, CARB 1992).

Validating model results remains "the most elusive of all the unresolved methodological problems associated with computer simulation techniques” (Naylor and Finger (1967) as quoted in Brewer 1973b). Identifying model performance requirements is a similarly daunting problem (Brewer 1973b). Performance requirements imposed by the EPA are based on past performance (NARSTO 2000b). The criteria may not be sufficiently strict to assure reliable decisions when, for example, uncertainties in the modeling are greater than the signal of interest (Fine et al. 2003).

Standard model performance assessments do not determine if "a model is performing well for the right reasons” (Russell 1997). Compensating errors are difficult to find, even if they are sought, but regulatory guidance requires no such pursuit. In summary, in the words of Russell (1997), "It is obvious that model evaluation continues to be a weakness in current regional modeling practice.”

\footnotetext{
$15 \mathrm{ppb}, \mathrm{PAQSM}$ may be unable to simulate ozone concentrations with an error of less than $\pm 15 \mathrm{ppb}$.

${ }^{34}$ For example, based on Tesche (1988), the EPA defines three classes of model performance. A Class A model has a maximum normalized error of 5 percent, a maximum normalized bias of \pm 5 percent and an accuracy unpaired in space and time of \pm 10 percent. Class B models have normalized error and bias of less than 35 and \pm 15 percent, respectively, and an unpaired accuracy of \pm 20 percent. Class $C$ models fail one of the performance standards for Class B models. In California, laws allow for Class A or B models to be used in air quality plans. For another example, see California Air Resources (CARB 1992).
} 
Ascher (1976) observed that a forecast need not be appraised or appraisable to have policy impact, especially if the forecast supports policy-makers' perceptions of the “correct” policies. Asher's observation may explain lack of rigorous performance requirements for PAQSM in regulatory applications.

\section{Representativeness}

Model complexity complicates the modeling task and constrains options for simulating observed episodes of high ozone. Although air districts maintain networks of continuous monitors, such routine observations provide insufficient inputs for data hungry PAQSM. Expensive intensive field studies are necessary to provide a robust observational database to execute models and to evaluate their performance. Intensive data gathering is too expensive to do routinely.

Consequently, selecting an ozone event to model is determined as much by the availability of observational data as by the event's relative severity. Typically, only one or a few ozone episodes are monitored sufficiently to provide input data for modeling. The "worst case” condition may not be represented. When this is the case, plans based on simulation provide limited evidence that standards will not be violated if more severe aerometric conditions are experienced. ${ }^{35}$ The Central California case involved full PAQSM simulation of one episode that occurred August 2-6, 1990 (CARB 1996b). The episode did not represent the most severe ozone conditions; peak observed ozone was

\footnotetext{
35 The standards actually allow for occasional violations of NAAQS. For example, noncompliance with the 1-hour ozone NAAQS is not triggered until the fourth observed violation over a three year period.
} 
$160 \mathrm{ppb}$, but the air district's design value was 170 ppb (CARB 1996b; SJVUAPCD 1994). ${ }^{36}$

One or a few episodes do not represent natural variability. Many aerometric conditions may lead to a violation of ozone NAAQS (for examples, see Lehrman et al. 1998; Blanchard 2001). Without modeling these myriad conditions, controls based on one or a few simulated events may be insufficient to attain the ozone NAAQS.

\section{Incommensurability}

Observational data used for model inputs and performance evaluation are not commensurate with model requirements (NARSTO 2000b). Models provide output in the form of pollutant concentrations resolved in space and time. This resolution, however, describes typical grid cells with dimensions of approximately $25 \mathrm{~km}^{2}$ in area and $50 \mathrm{~m}$ high. To test model performance the predictions are compared with numerous point measurements.

For example, the Bay Area Air Quality Management District uses a three-dimensional PAQSM with grids cells 100 meters tall and 25 square kilometers in area. The eightcounty region has an area of approximately two million square kilometers, thereby requiring about 80,000 cells to cover the entire surface of the region. The BAAQMD operates about 30 air quality monitoring stations that measure a range of pollutants. The model relies on 30 stations to provide input data for the ground level of the 80,000 grid cells, or about one station for every 2,700 cells. Furthermore, all of the monitoring

\footnotetext{
${ }^{36}$ The design value is defined as the fourth highest ozone concentration observed over a three-year period. The ozone NAAQS allows for an average of one violation per year, averaged over three years. Hence, the design value is the ozone level that triggers the nonattainment designation and determines the nonattainment classification (e.g., extreme, severe, serious, moderate).
} 
stations are located at or near ground level; there is only one continuous aloft

measurement (BAAQMD 1993). Clearly, the observational data used as inputs lack spatial resolution and coverage relative to the model.

\section{Opacity}

Model complexity leads to opacity because it is difficult to "see" what happens within the model. Two prime examples are that formal analysis of errors is not possible, and that lay people do not have an opportunity to understand what goes into or on in the model, or what comes out of it.

\section{Propagation of Error}

It is not feasible to propagate errors analytically in a PAQSM. When input parameters relate to outputs as simple sums or products, formal error analysis is possible using Gaussian error propagation, which is a special case of a set of statistical procedures to describe uncertainty called the Method of Moments. Mathematically, relationships

$$
\begin{aligned}
\mathrm{z} & =\mathrm{x}^{*} \mathrm{y} \quad \mathrm{z} \text { equals } \mathrm{x} \text { multiplied by } \mathrm{y} \\
& =\mathrm{x} / \mathrm{y} \quad \mathrm{z} \text { equals } \mathrm{x} \text { divided by } \mathrm{y} \\
& =\mathrm{x}+\mathrm{y} \quad \mathrm{z} \text { equals } \mathrm{x} \text { plus } \mathrm{y} \\
& =\mathrm{x}-\mathrm{y} \quad \mathrm{z} \text { equals } \mathrm{x} \text { minus } \mathrm{y} \\
& =\text { any function of } \mathrm{x} \text { and } \mathrm{y}
\end{aligned}
$$


Assuming $\mathrm{x}$ and $\mathrm{y}$ are uncorrelated, the error in $\mathrm{z}$ due to errors in $\mathrm{x}$ and $\mathrm{y}$ is:

$$
\mathrm{s}_{\bar{z}} \approx \sqrt{\left(\frac{\partial z}{\partial x} \mathrm{~s}_{\bar{x}}\right)^{2}+\left(\frac{\partial z}{\partial y} \mathrm{~s}_{\bar{y}}\right)^{2}}
$$

Where

$$
\begin{array}{ll}
\mathrm{s}_{\bar{z}}=\text { standard error of } z & \frac{\partial z}{\partial x}=\text { partial derivative of } z \text { with respect to } x \\
\mathrm{~s}_{\bar{x}}=\text { standard error of } x & \frac{\partial z}{\partial y}=\text { partial derivative of } z \text { with respect to } y \\
\mathrm{~s}_{\bar{y}}=\text { standard error of } y &
\end{array}
$$

The variables in a PAQSM, $\mathrm{x}$ and $\mathrm{y}$ in the above example, are related through complex mathematical functions that are far more complicated than the above example. It is not always possible to calculate the partial derivatives. Input variables are often correlated, not independent. Consequently, the analytical calculation of the standard error in PAQSM outputs due to errors in inputs is not possible.

Random sampling methods, such as Monte Carlo analysis, are used where formal error propagation is not tractable. The methods rely on numerous simulations using slight changes in input values to measure resultant changes in outputs. The sheer number of inputs makes comprehensive analysis difficult. Though it is possible to isolate the more influential variables to simplify the task, doing so does not account for correlated, uncertain inputs and does not assess internal bias in the model (Fine et al. 2003).

PAQSM uncertainty analysis methods are discussed in more detail in Chapter III PAQSM and Their Uncertainties. 


\section{Public Participation}

Air quality planning is required to be "transparent" to allow for public participation . However, model-based air quality planning does not truly give interested parties an opportunity to understand the modeling studies providing the rationale for planning decisions. Just as modelers and statisticians cannot calculate model uncertainties using formal analytic techniques, lay stakeholders are not given an opportunity to understand the modeling system. To learn about models and their uncertainties, lay stakeholders (and planners) are reliant on modelers to communicate their knowledge. This is a daunting task, especially so since capabilities to quantify the uncertainties are limited. In this respect, PAQSM modeling is an “immature” science (Ravetz 1999).

Lay stakeholders typically are not privy to the concerns associated with models’ simplified representations and inputs. Consequently, models are black box decision tools that are barriers to meaningful participation because citizens cannot peer into the modeling system and cannot evaluate critically the reliability of model output. The consequences of opaque decision tools warrant further examination in terms of the implications for public participation.

The 1967 and 1974 amendments to the Administrative Procedures Act (APA) delineated requirements for public participation in agency rule making. ${ }^{37}$ Subsequently, the APA incorporated other approaches to public notice and participation, including the Freedom of Information Reform Act of 1986, the Electronic Freedom of Information Act

\footnotetext{
${ }^{37}$ Sept. 6, 1966, P.L. 89-554, § 1, 80 Stat. 383; June 5, 1967, P.L. 90-23 § 1, 81 Stat. 54; Nov. 21, 1974, P.L. 93-502, §§ 1-3, 88 Stat. 1561, 1563, 1564.
} 
Amendments of 1996, and the Federal Sunshine Act. ${ }^{38}$ One example of the public disclosure requirements of the APA is:

"Each agency, in accordance with published rules, shall make available for public inspection and copying--

(A) final opinions, including concurring and dissenting opinions, as well as orders, made in the adjudication of cases;

(B) those statements of policy and interpretations which have been adopted by the agency and are not published in the Federal Register;

(C) administrative staff manuals and instructions to staff that affect a member of the public;

(D) copies of all records, regardless of form or format... ${ }^{39}$

The public notice and comment established in the APA has been termed the "decideannounce-defend” method (Jones 1997). The public may comment on air quality plans only after they have been formulated. In air quality management, agencies do hold hearings to get input for plans, but most public involvement is in the form of testimony or written comments on draft plans. Prior to the draft plan, public input typically involves the regulated community providing information to planners about their emissions causing activities and available technologies to abate them. Consequently, involvement by the lay, non-regulated public does not constitute meaningful public participation.

Seven elements for effective public participation are presented by Popovic (1993):

\footnotetext{
${ }^{38}$ Freedom of Information Reform Act (P.L. 99-570); Electronic Freedom of Information Act Amendments (PL 104-231); Federal Sunshine Act (68 ALR Fed 842).

395 USCS § 552 (1998).
} 
1. Education about the environment and the way it is affected

2. Accessible information

3. Voice in decision-making

4. Transparent decision-making processes

5. Post-project assessment

6. Systems for enforcement

7. Options for redress through independent "tribunals".

Model-based planning violates many of these elements. A commitment to modeling does not further public education, except to the extent that modeling results are communicated to the public. Although government agents provide materials for the public to educate themselves, the information is incomplete and does not provide enough detail about the science, or the modeling approach, to enable the public to criticize decisions. The important information is not accessible. Requirements of the APA and Popovic's criteria for information disclosure and transparent processes are violated when public agents use opaque tools without communicating the tools' limitations and responses to those limitations. Models remain opaque.

Some modeling studies involve the formation of "technical” committees that include stakeholders. They are far from tribunals, rather collections of academicians or professional consultants serving as impotent advisors. Non-technical people and public interest groups are not typically invited to serve on such advisory committees. For example, air quality studies in Central California are managed by policy and technical committees. Although participation has changed over the years, the committees are comprised entirely of government agents, academics, and members of the regulated communities (SJVAQS 1996, Appendices B and D).

Innes (1998) sets forth criteria for rational communicative planning: 
- All stakeholders have representation at the negotiating table, and are fully and equally informed and empowered.

- Negotiators endeavor to maintain good, honest and open discussion.

- Discussion allows all claims, assumptions and constraints to be tested for their factual basis (i.e., scientific verifiability), clarity (e.g., non jargonladen), and comprehensiveness.

- Speakers are evaluated against criteria for honesty, sincerity, and expertise (i.e., experience and credentials to make the statements that they make).

- Negotiators seek a consensus.

Without the invitation or resources to participate meaningfully when invited, citizens and environmental advocacy groups do not have an opportunity to participate to the same extent as the regulated community. Furthermore, they would not likely have the capacity to do so should they be given the opportunity because participation requires the expertise necessary to engage the modeling. The complexity and opacity of the models, decideannounce-defend process, and the failure of modelers and planners to communicate assumptions and uncertainties violate every criterion set forth by Popovic and Innes.

The decide-announce-defend approach to public participation does not allow for public influence at the critical juncture of decision making (Popovic 1993). Timing is an essential component of effective participation. The citizen role during the "defense" stage can delay but rarely can it fundamentally change a decision (French 1993). At best, decide-announce-defend allows for informing, consultation and, perhaps, placation of the public per Arnstein’s “ladder” of public participation in community planning (Arnstein 1969), as shown below in Figure II-3. None of these functions, however, extend beyond various degrees of tokenism. 
Figure II-3: Arnstein's Ladder of Public Participation

\begin{tabular}{|c|}
\hline Citizen Control \\
\hline Delegated Power \\
\hline Partnership \\
\hline Placation \\
\hline Consultation \\
\hline Informing \\
\hline Therapy \\
\hline Manipulation \\
Nogrees of Tokenism \\
\hline
\end{tabular}

\section{Subjectivity}

\section{Performance Criteria}

If models are inherently uncertain, only capable of local validation, and not falsifiable, how are we to determine if a model is acceptable for use in air quality planning? The answer does not lie with Boyle’s experimental science even though the air quality community universally states that adequate performance evaluation is central to determining model output credibility (Roth et al. 1989). Rather, model acceptability is a socially derived norm. The EPA and CARB issued guidance on how to use models, and what constitutes acceptable performance (EPA 1987; 1991; 1992a; 1993b; 1996a; 1996b). Performance criteria, however, have a questionable scientific basis because they are a compromise between what is needed and what is reasonable to expect given performance reported in earlier studies (NARSTO 2000b). Therefore, the science of model acceptance through performance evaluation is a social construct that relies on accepted norms rather than, for example, an assessment of signal-to-noise ratios. 


\section{Judgments}

Another aspect of subjectivity in PAQSMs is the need for judgment by the modeler. Assumptions are necessitated by inability to know the future or to represented physical and chemical processes mathematically. In his reviews of population, economic, energy, transportation, and technological forecasting, Ascher (1976) found assumptions to be the greatest determinant of forecast accuracy. Examples of key assumptions used in PAQSMs include selecting an air quality episode to model, estimates of future emission growth, and the effectiveness of untried emissions controls.

More generally, Jager (1998, page 143) noted "what science is, and how it is used, is not straightforward” and summarized the observations of Jasanoff (1995):

- Scientific inquiry into observed phenomenon does not always lead to the same explanation

- Scientific conclusions about the reasons for particular natural phenomenon are not always based on the same explanations

- It is not necessarily knowledge endorsed by science that compels action

- Whereas too little science may impede action, so too might too much

- Whereas groups may agree that a problem exists, they cannot always agree about how the problem should be conceptualized for scientific investigation.

These observations reveal the misplaced optimism in the positivist presumption that there is only one truth out there just waiting to be discovered by objective researchers.

\section{Information Needs Revisited}

A third subjective element of PAQSM involves the use of their output. Some models are incapable of providing information in the form that decision-makers need to manage multi-attribute problems. Planners attempting to balance many considerations (e.g., 
achieving air quality goals, minimizing emissions reduction costs, appeasing stakeholders with divergent objectives) need more information than estimates of pollutant concentrations provided by the models (for example, see Winstanley et al. 1998). Decision-makers may consider economic, social and political concerns, but PAQSMs provide only information about the estimates of changes in air quality resulting from changes in pollutant emissions. ${ }^{40}$ Morgan and Henrion (1990) provide several examples: decisions based on cost-benefits analysis require information about the change in air quality (e.g., decrease in peak ozone) per dollar spent for a unit of emissions reduced; risk management decisions need to be informed by the change in morbidity per dollar invested on emissions reduced; whereas risk assessment is concerned with change in morbidity per total emissions reduced. Unfortunately, PAQSM provide only information about the change in air quality per increment of emissions reduction.

\section{F. Air Quality Planning Challenges}

The uncertain, opaque and subjective aspects of models and their applications are enough to undermine belief in their objective use. Layered upon these concerns is the nature of the air quality planning task. Air quality planners face many challenges. In addition to the lack of progress in meeting longstanding ozone NAAQS, the EPA promulgated new standards. Economic expansion and population growth creates new and expanding sources of emissions. Inadequate or unfamiliar planning tools, as well as untried emissions control technologies, confound planning efforts.

\footnotetext{
${ }^{40}$ Integrated assessment models have been developed to inform decisions using economic criteria. Although EPA has embraced models, such as REMSTAT, to simulate the ozone reduced per unit cost, they add an additional level of uncertainty by extending the simulation to socioeconomic processes. Critiquing integrated assessment models will be a natural extension of my dissertation.
} 
Growth in both population and economic activity continues to offset the gains made by emissions controls. EPA estimates that anthropogenic nitrogen oxides emissions increased three percent since 1991 (EPA 2001). In another example, individual automobiles are far cleaner today than in the past, but since 1970, total vehicle miles traveled increased approximately 150 percent (EPA 2001).

Many areas with historically clean air are becoming polluted. Over the past ten years, average ozone levels increased four percent in National Parks (EPA 2001). For the first time since measurements began, ozone concentrations in rural areas have, on average, exceeded urban concentrations for three consecutive years (EPA 2001).

Historically dirty areas, such as Los Angeles, Atlanta, and the northeastern U.S. still regularly experience violations of ozone NAAQS (EPA 2002). Air has gotten cleaner in these areas, but it is not evident that model-based planning is the cause. Obvious actions, such as vehicle emissions standards and controls imposed on large point sources, yielded benefits. In the San Francisco Bay Area and the San Joaquin Valley, progress achieved in the 1970's and 1980's has not been so easy to continue in the 1990's. In fact, the two air basins have shown little progress toward ozone goals during the past decade. (Air quality conditions in Central California are detailed in Chapter IV - Central California Air Quality.)

In addition to historically elusive standards, such as the 1-hour average ozone NAAQS, the Environmental Protection Agency recently promulgated new NAAQS for 8hour average ozone and fine particulate matter (EPA 1997a). EPA also promulgated new standards for regional haze (Fed. Reg. 1999b). These standards will require new or more 
stringent controls on as yet unspecified emissions sources. In 1997, EPA used monitoring data from 1993 to 1995 to estimate that 250 counties, home to 83 million people, would have violated the 8-hour ozone standard (EPA 1997a). More recent monitoring from 1998 to 2000 indicated that over 300 counties currently violate the 8hour ozone standard (EPA 2001), including more rural areas.

Increasing levels of background ozone concentrations, the 8-hour ozone NAAQS, and the regional haze rule create a greater impetus to control emissions from sources in rural areas. Controls may be required on heretofore sparsely controlled area (“nonpoint”) sources, including diesel trucks and agricultural operations. ${ }^{41}$ Controlling new sources represents yet another challenge to existing planning practice. Nonpoint emissions are harder to identify and estimate. By definition, point sources are large, stationary and, thankfully, few in number. Nonpoint sources, such as agricultural water pump engines or livestock waste, are numerous, small, and often hard to locate and quantify. Enforcing controls on these sources will be difficult unless they have strong incentives to comply. Furthermore, industries subject to these new controls, such as agriculture, have powerful political connections and have historically been exempt from clean air laws.

These challenges layer upon longstanding issues, such as the preconceived notion of a tradeoff between environmental quality and economic vitality. In Central California, the agricultural and petroleum industries, amongst others, characterize emissions controls as economic threats. Furthermore, recent emphasis on equitable sharing of the costs of controls and the impacts of poor air quality, largely through the principles of environmental justice, further complicate planning efforts. 
Given these myriad challenges facing air quality planners, and the limits of their most tool, the concept of a trans-scientific problem (Weinberg 1972) applies well. Air quality planning decisions "hang on the answers to questions which can be asked of science and yet which cannot be answered by science. [italics added by Weinberg]”. It is what Rittel and Webber (1973) call a "wicked" problem because a host of social goals and values must be optimized, and there are likely to be big losers (e.g., those charged with reducing their emissions, those who breath polluted air). Competing goals to be managed in planning decisions include:

- continued economic activity and growth

- sustaining at least the status quo quality of life

- achieving air quality within time frames defined as the result of the social process and codified in laws

Values influence these objectives. Given the myriad values of society, the solution set is undefined and the facts of the problem are partly determined by how the problem is framed.

\section{Post-Normalcy}

The air quality planning process delineated by laws and regulations is based on a paradigm that treats problems as solvable through reductionist and atomistic methods of normal science. Unfortunately, air quality planning is not a problem for what Kuhn (1962) calls "normal science" - the process of fact gathering "for further articulation or specification [of a paradigm] under new or more stringent conditions.” Instead, the

${ }^{41}$ For an example from Central California, refer to (SJVUAPCD 2001). 
development of technical information to inform policy deliberations is a form of "science for policy”, which is very different than science for pure knowledge (Jasanoff 1990).

The more realistic depiction of the air quality planning problem is one with debatable facts, contextualized values, multiple decision objectives with high stakes, and significant uncertainties; considerations of science for policy that has been termed "post-normal" science (Funtowicz and Ravetz 1992). In post-normal science the assumption of unconditioned and absolute scientific evidence does not exist. Problems instead require consideration of the qualitative, subjective aspects of information that appears to be strictly quantitative in nature.

Air quality planning can be described in all of these ways, as a wicked, transscientific, post-normal scientific problem. These perspectives consider three key factors: high stakes, multi-attribute decisions, and considerable uncertainty. Summarizing their experience with acid deposition control planning, Winstanley et al. (1998) wrote,

In an ideal world, objective science provides the foundation for decision making. In reality, policy and politics influence science and science is only one ingredient in decision making.

Concerned with what policy analysts and planners actually do, Fischer and Forester (1993) posit that any such analyses or plans are "practical processes of argumentation”. The argumentation is designed to address the multiple, competing objectives of decisionmaking in the face of uncertainty and high decision stakes. Dimensions of interest include identifying facts, navigating the political setting, weighing costs and benefits, as well as acknowledging, developing, and communicating values. Thus, to understand the apparent contradiction of analyses that manage simultaneously political concerns and 
scientific rigor, Fischer and Forester (1993, page 3) define a research agenda that is similar to the one undertaken in support of this research:

To understand what policy analysts and planners actually do, we need to assess the political conditions in which analysts work. We need also to probe the daily politics of problem definition and framing,... of rationality in constant tension with sources of bias.

The far more complex relationship between technical information and other inputs in decision-making is depicted in Figure II-4. In addition to the influence of politics in the planning space, all of the components interact. These interactions obscure the relationship between technical information and decisions.

Figure II-4: Actual Role of Models in NAAQS Planning

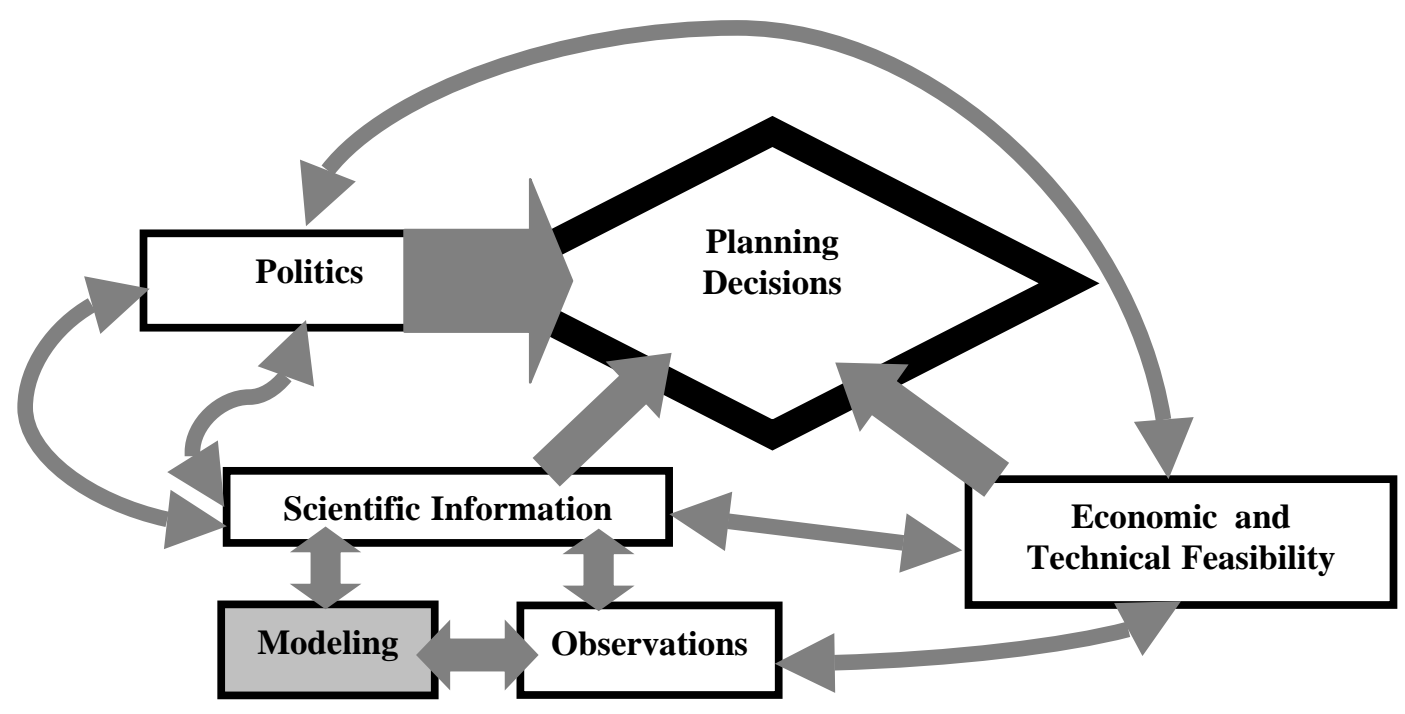




\section{Decision Bias}

There is a tendency for SIPs to underestimate the amount of emissions controls necessary to meet ozone standards. ${ }^{42}$ The under prediction may be due to bias or error, or a combination of the two. Air quality improvement plans purportedly rely on a "best" estimate of necessary emissions reductions, usually produced by a PAQSM. In an unbiased world some error - both under and over estimates - would be expected, but there should be no bias. The tendency toward underestimates must be attributed to bias.

The problem of decision bias is a sign that progressive governance has not worked for air quality planning. Evidence of decision bias, as provided by unsuccessful SIPs, complements an existing body of theoretical and empirical research suggesting that neither scientists, nor the information they produce, nor the policy makers using this information are objective.

What has gone wrong? The first possibility, one emphasized in the discussion of the limits of models, is that our science was inadequate; that models we trusted were insufficiently accurate. Consequently, planners reliant on models made misguided decisions. If this is the case, then more careful use of the models is our solution to clean air.

\footnotetext{
${ }^{42}$ Failure to meet goals may be due to an under estimate of controls needed or overestimates of either the rate of implementation or effectiveness. In addition, plans may over control the wrong pollutants (e.g., VOC controls instead of $\mathrm{NO}_{\mathrm{x}}$ controls in pursuit of ozone standards). The problem can be viewed as one with four scenarios, assuming that the "correct" decision is to control pollutant B: 1) over control of pollutant A, under control of pollutant B; 2) under control of pollutants A and B; 3) under control of pollutant A, over control of pollutant B; and 4) over control of pollutants A and B. Planning experience shows that scenarios 1 and 2 are common, and scenarios 3 and 4 are rare. If scenarios 3 and 4 were not rare, then more areas would attain ozone standards.
} 
Unfortunately, given broader consideration of models' limitations and challenges facing air quality planners there is another possibility: that politics overwhelmed the uncertain science, and the net result of political forces were insufficient emissions controls. Short on resources and unable to generate incontrovertible scientific justification, planners may be driven by what Greenberger et al. (1976) termed the "issueattention cycle”, whereby political will and public opinion has more bearing on planning decisions than technical evidence. Furthermore, it is possible that the scientists themselves, and hence the modeling, were influenced by the politics.

In discussing Mazur's The Dynamics of a Technical Controversy (1981), Aronson (1984, pages 16-17) wrote,

A notable alternative to the traditional view of scientists is that of Mazur, who, attempting to mediate a dispute between experts over the health risks of extremely high voltage electrical transmission lines, found that while it was possible - though difficult - to separate fact from value in the arguments raised by the adversaries, it was impossible - for political reasons - to persuade them to reconcile their differences. Mazur concludes that, when scientists disagree over technical issues, the dispute is often primarily "over political goals and only secondarily concerned with the veracity of scientific issues which are related to these goals," thereby suggesting that scientists do indeed act as social problem "activists."

Is the bias observed in SIPs due to subjective use of models? Are modelers and planners using modeling results as "social problem activists"? This research gets at these questions. To the extent that the answers is a universal yes, then it is all the more clear that the presumed objective use of models was wishful thinking, and that the way to improve the process requires more than just model uncertainty explication and reduction. 


\section{G. What is the relationship between science and policy?}

Many of the issues discussed above bring into question the interactions of science and policy. In reviewing the literature about this interaction, Jager (1998) identifies two descriptions. First, science and policy may be understood as existing in distinct realms. A second description builds from Weinberg's concept of trans-science, whereby policyrelevant science questions must be addressed using non-scientific methods. Extending Weinberg's definition, regulatory science involves political or legal pressures and timelines that call upon science for evaluation and assessment rather than new research (Jasanoff 1996b). Due to deadlines for decision-making that truncate research opportunity, "pragmatic regulatory policy decisions” are achieved through a "mutual construction" of science and policy, even though decisions may subsequently be presented as being based on purely scientific considerations (Jager 1998, page 144). This last description, that of mutual construction, begins to offer the type of nuance necessary to understand the process of model-based air quality planning.

Why model? Model for whom? Are decisions based on models?

The search for improvements to model-based decision-making can be informed by questioning the purposes of modeling. Receptivity to requests for model uncertainty analyses will depend on who may be served or disadvantaged by the uncertainty information. More important, the extent to which uncertainty information is ultimately used depends integrally on its beneficiaries.

Brewer (1973b) argues that divergent participant interests may be understood as different foci of attention and modes of viewing the world. Foci are distinguished by intellectual attitude, time scales of interest, and the scale of units of analysis. Modes may 
be manipulative, reflective or explanatory. Air quality model uses can be understood as strictly one mode, or as combinations. Where combinations occur, the necessity for and purposes of uncertainty analyses may be controversial. A participant operating in the explanatory mode may consider uncertainty analyses essential for causal and deductive assessments. If the purpose of modeling is manipulative, however, uncertainty analyses may be considered extraneous or inconvenient.

Brewer (1973b) identifies several policy applications for modeling:

- Validate a decision already made

- Slow down or avoid making a decision

- Descriptive clarification (determine the present state and past trends)

- Unconditional forecasting

- Normative specification (create and examine various end states)

- Program evaluation

Many of these applications differ from hypothetico-deductive research and planning. The first and second applications are clearly manipulative; the remaining applications may be primarily explanatory or reflective, but may also be manipulative. For example, some explanatory modes may be manipulative if they are based on research questions formulated to exclude other potential avenues of investigation or, at least implicitly, emphasize some concerns over others. Decision validation and aversion are the only two applications seeking model uncertainty information, but for very different strategic reasons. 
Writing about the uses of science in public decision-making, rather than simulation modeling specifically, Ozawa (1991; 1996) describes four uses for science: (1) discoverer, (2) mechanism of accountability, (3) shield, or (4) tool of persuasion. When used for discovery, science is produced by an apolitical, disinterested investigator who, in seeking to understand the physical world, identifies something worthy of broader social action. Science can be a mechanism of accountability when an independent entity makes decisions on purely scientific grounds. The legal construct of the requirement for a modeled "attainment demonstration" in air quality plans derives from the idea that "[a]s long as agency decision-makers were constrained by technical experts' interpretations of the physical conditions and alternative actions, Congress assumed that raw politics would be constrained" (Ozawa 1996; page 224). Science may be used to defend or to question controversial decisions. An example of the latter is citing air quality models in court hearings as providing the best available evidence upon which decisions were based. Last, science may be used to persuade once its authority is established as legitimate.

Greenberger et al. (1976) offer three lessons about how prediction products may not be as important as the process, finding that some results are politically salient, whereas others are not, and that the political setting in which the model is presented may be decisive in how the model is perceived by policymakers. Furthermore, they also conclude that the organizational framework in which a model is developed can be a crucial determinant of its usefulness. These three lessons explain the heavy reliance on PAQSM for planning because their policy applications and rhetorical utility make them politically palatable, if not preferred. The political setting influences receptivity to model results. 
When used in the manipulative mode (e.g., to justify a predetermined decision), modeling results must not bring into question agendas of those in control of modeling. The same will be true for the results of model uncertainty analyses, except that the effect is secondary. For example, if a decision-maker concludes “yes” prior to modeling, and the modeling result subsequently indicates "no", then the model is likely to be rejected or, at least, scrutinized. Uncertainty information will be pursued and emphasized because it downgrades the veracity of the modeling result from "no" to "maybe". When applied for risk assessment, the manipulative uses of uncertainty are reduced because a more honest form of information is provided.

\section{H. Conclusions}

This chapter discussion forwards several claims:

1. Objective information is needed for environmental decision-making.

2. Scientific research provides information per the needs of decision-makers.

3. Modeling research is uniquely capable of producing some needed information.

4. Modeling applications are not purely objective, due to limitations of uncertainty, opacity and subjectivity.

5. Air pollution, like many other environmental problems, cannot be managed solely through scientific research because it involves significant, inequitably-shared risks and burdens, competing values, and contested science.

A case is made for the need to address the limits of PAQSMs, in light of the challenges facing air quality planners, prescriptions are two-fold. First, there is need for explication, communication and, where possible, quantification of uncertainties in modeling. As noted briefly herein and discussed extensively in the next chapter, some methods are available, but they are incomplete. Nor have they been put to the task in any comprehensive manner. 
The second set of prescriptions emphasize planning processes that consider explicitly non-technical components of the problem through consensus-based, pluralistic means that employ a set of principles for generating and communicating information. In discussing process-related suggestions, it is evident that more consideration of the social aspects of modeling and policy-making must be understood. 


\section{PAQSM AND THEIR UNCERTAINTIES}

\section{A. Introduction ${ }^{43}$}

This chapter describes photochemical air quality simulation models (PAQSMs) and their uncertainties. It introduces analyses that are or may be performed on models and modeling studies to evaluate and estimate their uncertainties. The limitations of model performance criteria to motivate the presentation of methods for a more comprehensive evaluation. Last, experiences with modeling uncertainties and their evaluation are reviewed.

\section{B. Ozone Complexity and Models}

\section{Ozone Complexity}

The complexity of ozone formation compels air quality management agencies to use photochemical air quality simulation models (PAQSMs) to plan how to bring air basins into compliance with standards set to protect human health and welfare, most notably the National Ambient Air Quality Standards established by the FCAA.

Planners must decide what emissions controls to implement in pursuit of air quality standards, as well as the location and timing of controls. Models are used explicitly to generate information per the needs of the planner. Most important is the models ability to simulate the interactions of complex nonlinear chemistry, meteorology and pollutant emissions, and to forecast air quality in the future.

\footnotetext{
${ }^{43}$ Parts of this chapter are based on Fine et al. (2003).
} 


\section{Increased Reliance on Models for Regulatory Decision-making}

Use of sophisticated PAQSMs for air quality management has increased dramatically during the past three decades (Roth et al. 1993). Figure III-1 shows the evolution of models from the research and development stages to widespread regulatory application. The pinnacle of legal requirements for modeling is the FCAA Amendments of $1990 .{ }^{44}$

Figure III-1: Growth in the Regulatory Application of Air Quality Models (Based on Roth et al. 1993; Roth et al. 1997)

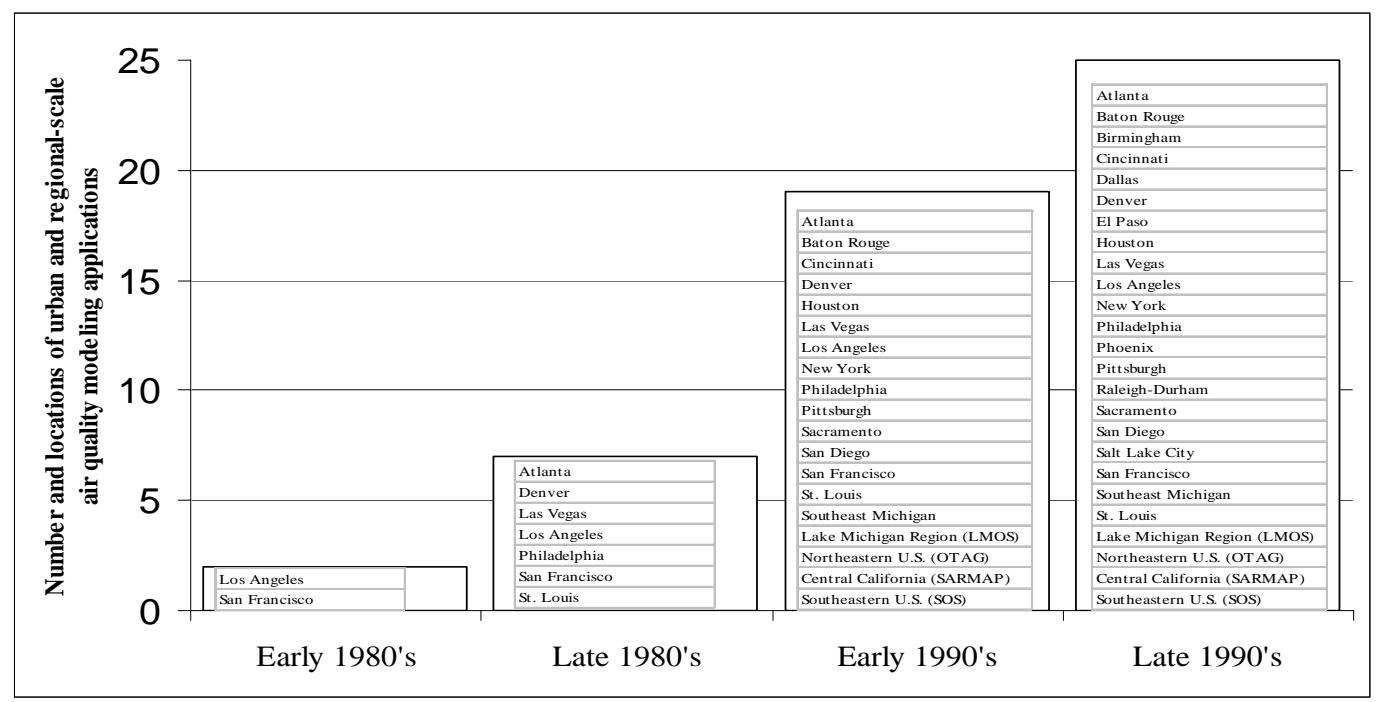

\section{Uses of Uncertainty Information}

Uncertainty information is needed by planners who must decide what emissions controls to implement in pursuit of air quality standards. The fundamental question underlying a plan to meet ozone standards is:

How much must current and anticipated future $\mathrm{NO}_{\mathrm{x}}$ and VOC emissions be reduced to meet the ozone NAAQS by a specified deadline?

Models are used explicitly to generate information to meet the needs of the planner. Most important is the model's ability to simulate the interactions of complex chemical, meteorological and pollutant emissions processes, and to estimate air quality in the 
future. If used wisely, simulation is an "indispensable tool for predicting the outcomes of alternative policies” (Stokey and Zeckhauser 1978).

During SIP development, PAQSM are used to simulate an observed violation of the ozone NAAQS concentration threshold. Once the "base case” simulation meets performance criteria specified by oversight agencies ${ }^{45}$, the PAQSM is rerun with scenarios representing emissions reductions from hypothetical controls. The modeling is said to "demonstrate attainment” when modeling results indicate that planned controls will reduce ozone concentrations to below the standard if the meteorological conditions in the simulated episode are experienced again. Similarly, changes in distant, upwind emissions sources may be simulated to evaluate the significance of pollutant transport. As discussed in the remainder of this paper, modeling output may also be used to characterize uncertainties.

If model capabilities fall short of demands, decision-makers still need information. They may rely on judgments to span information gaps and to justify decisions. This subjective aspect of planning often leads to controversy. There is also legal impetus to use models to demonstrate that decisions are neither arbitrary nor capricious. ${ }^{46}$ Planners rarely have other tools or information available to provide the bases for decisions. Model

${ }^{44}$ See discussion of Modeling Requirements of Ozone Attainment Demonstration Plans in Appendix B.

${ }^{45}$ EPA sets performance criteria to be met before using a modeling simulation for SIP planning (see EPA 1996). In California, additional criteria are set by CARB (1992).

${ }^{46}$ In Chevron U.S.A. vs. Natural Resources Defense Council (NRDC), NRDC challenged EPA's approach for applying emissions controls to meet the requirements of the Clean Air Act Amendments of 1977. The U.S. Supreme Court supported EPA, stating, "the EPA should have broad discretion in implementing the policies of the 1977 Amendments [of the FCAA]" (467 U.S. 837). The opinion further states,

If Congress has explicitly left a gap for the agency to fill, there is an express delegation of authority to the agency to elucidate a specific provision of the statute by regulation. Such legislative regulations are given controlling weight unless they are arbitrary, capricious, or manifestly contrary to the statute. 
uncertainty information can reduce the need for judgment and make judgments explicit for the purposes of public debate.

Depending on the decision criteria ${ }^{47}$, decisions may be facilitated by more complete model uncertainty information. Making decisions under uncertainty, planners should consider the likelihood that their plans will yield air quality goals once implemented. They need to assess risk, which is the chance of suffering harm or loss (Webster's 1994). The question that comes to mind is:

What is the likelihood that ozone NAAQS will actually be met when the model indicates that planned emissions reductions will yield attainment?

The answer to this question is a probabilistic statement. Using modeling output as well as information about output uncertainty facilitates risk assessment. In addition to risk assessment and management, there are at least six uses for model uncertainty information:

1. Satisfy the regulatory requirement to demonstrate acceptable model performance.

2. Enable planners to estimate the probability of not meeting goals even though model projections indicate the goals will be met.

3. Identify situations where model uncertainties are greater than the needed air quality improvement. For example, modeled responses of ozone concentration to changes in emissions may be the same magnitude or less than accompanying uncertainties. Having such knowledge and understanding is essential to interpreting results.

4. Suggest alternative control plans that may produce comparable air quality improvement within the range of uncertainty of the modeling results.

${ }^{47}$ Examples of decision criteria are given by Morgan and Henrion (1990), including:

- Cost-benefit: Deterministically comparing the costs and benefits of alternatives, or doing so probabilistically by incorporating uncertainty and then comparing expectations of costs and benefits.

- Cost effectiveness: Choosing least cost routes to goals that are not necessarily based on economic considerations.

- Zero or bounded risk: Decisions reducing or altogether prohibiting undesirable outcomes without consideration of costs or benefits. 
5. Inform general planning and resource allocation. For example, guide the planning of large field studies by identifying what data to gather, as well as where and when to gather them.

6. Set research priorities to improve the characterization of complex atmospheric processes by using both uncertainty and sensitivity information to identify key PAQSM components needing improvement.

\section{PAQSM Defined}

A photochemical air quality simulation model is an attempt to

...describe a dynamic, physical phenomenon by mathematical relationships which, when combined with accurate input data, imitate the real system (Cleary 1976).

A PAQSM is a mathematical representation of physical and chemical processes occurring in the atmosphere and at the atmosphere/land interface, including emissions, diffusive and advective transport, chemical transformation, and deposition. A PAQSM integrates our knowledge of the spatial and temporal evolution of gaseous and particulate constituents in the atmosphere. In addition to emissions and atmospheric processing, it represents the physical system comprised of topography (e.g., mountains), surface characteristics (e.g., land use and land cover) and meteorology (e.g., winds, temperatures, clouds). The PAQSM domain may range from an urban airshed to a regional to a continental-scale area.

\section{Models of a PAQSM}

The key components of PAQSM are shown in Figure III-2. They are meteorological, emissions, and air quality models. ${ }^{48}$

\footnotetext{
${ }^{48}$ The entire photochemical air quality simulation modeling system is referred to as a "PAQSM" and the
} 
Figure III-2: Component Models of a Photochemical Air Quality Modeling

System

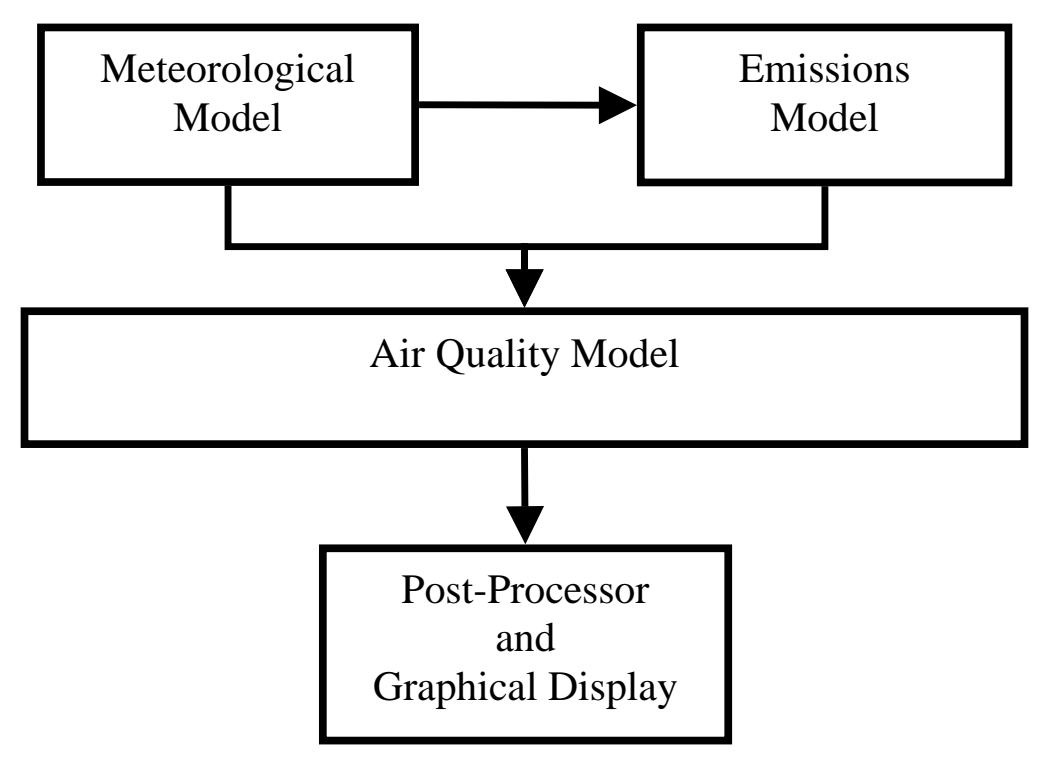

The meteorological model uses pertinent information to generate meteorological “fields" - wind speed and direction, temperatures, humidity - that are inputs to the emissions and air quality models. The emissions model calculates emissions from natural and anthropogenic sources. The air quality model contains descriptions of the physical and chemical transformations, transport and numerical solution algorithms.

Many tiers and types of models are embedded in the components of the PAQSM depicted in Figure III-3. For example, the emissions model component will include formulae for estimating emissions from stationary point, area, on- and off-road mobile, and biogenic emissions. In addition, a geographic information system may be used to 
organize and manipulate spatially resolved data, and post-processing systems may summarize and display results graphically.

Mathematical description of the dynamics of gases and aerosols in the atmosphere is achieved using conservation equations for mass, momentum, and energy. Pollutant transport and transformation is tracked temporally and spatially using the AdvectionDiffusion Equation (ADE).

\section{Figure III-3: Advection-Diffusion Equation}

$$
\frac{\partial \mathrm{c}_{\mathrm{i}}}{\partial t}=-\frac{\partial}{\partial \mathrm{x}_{\mathrm{j}}}\left(\mathrm{u}_{\mathrm{j}} \partial \mathrm{c}_{\mathrm{i}}\right)+\frac{\partial}{\partial \mathrm{x}_{\mathrm{j}}}\left(\mathrm{K}_{\mathrm{j}} \frac{\partial \mathrm{c}_{\mathrm{i}}}{\partial \mathrm{x}_{\mathrm{j}}}\right)+\mathrm{R}_{\mathrm{i}}+\mathrm{S}_{\mathrm{i}}+\mathrm{L}_{\mathrm{i}}
$$

$$
\begin{aligned}
& =\text { Advection }+ \text { Turbulent Diffusion }+ \text { Reactions }+ \text { Sources }+ \text { Removal } \\
& \text { for } j=x, y, z \text { dimensions } \\
& \text { for } i=1, \ldots, n \text { pollutant species }
\end{aligned}
$$

The ADE describes how the time rate of change of concentration, $c_{i}$, of the $i^{\text {th }}$ pollutant equals the net changes due to five processes:

- Advection by the mean wind components, $\mathrm{u}_{\mathrm{j}}$

- Turbulent diffusion characterized by gradient transport using eddy diffusivity, $\mathrm{K}_{\mathrm{j}}$

- Production and destruction of $\mathrm{i}$ through chemical reactions, $\mathrm{R}_{\mathrm{i}}$

- Addition of $\mathrm{i}$ by emission sources, $\mathrm{S}_{\mathrm{i}}$

- Removal of $\mathrm{i}$ at the surface or by other physical processes, $\mathrm{L}_{\mathrm{i}}$

Each pollutant is described with an ADE. The result is a set of coupled, nonlinear partial differential equations that satisfy conservation relationships in a turbulent flow. 
Coupling occurs between pollutant species in the reaction term and is an important source of the nonlinearity of the system. The equations require numerical, not analytic, solution.

For each time step, $\Delta t$, of a simulation pollutant concentrations in grid cells change as they are gained (lost) through inflow (outflow) and chemical formation (destruction). Transport terms include wind-induced advection and turbulent diffusion. Emissions are an inflow at the ground level or, for large point sources, into a horizontal layer aloft. ${ }^{49}$ Refer to Russell (1997) or Seinfeld and Pandis (1998) for detailed reviews of model formulation.

\section{Types of PAQSM}

The two types of photochemical air quality simulation models used today are distinguished by their frames of reference. Eulerian constructs overlay onto the modeling domain a three-dimensional (3-D) grid system of a particular resolution with a fixed frame of reference. Trajectory formulations start with a frame of reference that moves a control volume ${ }^{50}$ over space and time using a prescribed meteorological variable, such as wind velocity. ${ }^{51}$

Eulerian models are the state-of-the-science and require the least restrictive assumptions. In the United States, they are the models of choice for regulatory applications. However, they are computationally and data intensive. Oftentimes, there are insufficient data to support Eulerian model applications. Computational requirements

\footnotetext{
${ }^{49}$ Typically, emissions from tall stacks occur above the surface layer, so they are assumed to inject into a horizontal layer that is above the surface.

${ }^{50}$ The control volume is often assumed to correspond to a particular air mass.

${ }^{51}$ Trajectory models are often referred to as Lagrangian formulations. The term is not used here because, strictly speaking, trajectory models are a simplification of Eulerian models that treat horizontal transport and turbulent diffusion as negligible.
} 
may be a concern when considering the execution of a large number of simulations to fully explore model sensitivity or control strategy issues.

Trajectory models are less demanding computationally, require less input data, are a simpler representation of the physical system, and provide less information about the spatial character of pollutants. They use averages of observed wind speed and direction to transport a single air mass over space and time and, thus, have limited ability to represent complicated pollutant transport situations. The domain may be described as a single box, a zero-dimensional model (0-D), or as many boxes stacked vertically, a one-dimensional model (1-D), to allow for vertical mixing and pollutant concentrations that vary vertically. The simplifications of trajectory models render them inappropriate for simulations extending beyond the period over which assumptions are valid. In particular, the integrity of the air column or boxes is violated over space and time in the presence of significant wind shear.

Many model uncertainty evaluations have been conducted using trajectory models because of their computational and input simplicity advantages, though recent efforts have used Eulerian models (Milford et al. 1989; Milford et al. 1994; Bergin et al. 1995; Yang et al. 1997; Bergin et al. 1998; Hanna et al. 1998; Hanna et al. 2001). It is easier to work with trajectory models, but their simplifying assumptions are violated more easily. Eulerian models are the dominant tools used for regional-scale air quality planning. ${ }^{52}$ There is a trend toward simulating larger domains (i.e., regional and sub-continentalscales) and longer time periods (i.e., an entire ozone season lasting several months).

\footnotetext{
${ }^{52}$ Point source dispersion models enjoy wide application for individual project permit applications. As this paper is concerned with regional-scale air quality planning, dispersion models are not reviewed.
} 
Using larger domains reduces errors associated with boundary conditions and allows for the examination of pollutant transport over regional scales. Modeling a full ozone season addresses concerns about the representativeness of simulating only one or a few multiday ozone episodes.

\section{PAQSM Inputs}

PAQSM inputs may be categorized according to meteorology, emissions, topography, grid structure, and atmospheric concentrations specified at the outset of the simulation and at the boundary of the domain (Russell 1997). The delineation of outputs and inputs is confounded by intermediate products, since output from one model of a PAQSM can be input for another. Aerometric observations may be used to specify initial and boundary conditions, and to provide comparative data for model performance evaluation. The emissions and chemistry models rely on output from the meteorological model because chemical reactions and some emissions rates vary with actinic flux and air temperature. ${ }^{53}$ Meteorological fields, emissions estimates, and chemical kinetics and rates (i.e., chemical mechanism) are inputs for the air quality model.

\section{PAQSM Outputs}

Outputs are defined here as information produced by a PAQSM, notably estimated pollutant concentrations. Modelers use output, as well as intermediate products, to evaluate model performance, whereas policy-makers are interested in predicted pollutant concentrations to determine the magnitude of emissions reductions needed to meet air quality standards. The review by NARSTO (2000b) identified four types of output:

\footnotetext{
${ }^{53}$ Although the dependency of emissions on meteorology is treated in PAQSM, feedback effects of
} 
- Ozone: Concentrations estimated in space and time resulting from estimated historical, current or anticipated emissions.

- Ozone precursors: Concentrations of precursor (e.g., $\mathrm{NO}_{\mathrm{x}}$, hydrocarbons) or indicator species (e.g., CO) estimated in space and time. These chemical species are relevant and important, as they must be simulated accurately to conclude that the accuracy of ozone predictions is due to correct representation of relevant processes.

- Ozone sensitivity: Changes in ozone due to changes in precursor emissions or concentrations at the boundary.

- Transport: Relative contributions from distant and local precursor emissions to peak ozone concentrations.

\section{E. PAQSM Uncertainties}

The uncertainties described in this section are found in the component models of a PAQSM, and they interact both within and across models. For organizational convenience uncertainties pertaining to inputs, formulation, variability, and the use of results are discussed separately.

\section{Uncertainties in Inputs}

\section{Emissions}

Estimates of emissions are among the most uncertain inputs of PAQSM (NRC 1991; NARSTO 2000b; Russell 1997). Emission accuracy determines estimation accuracy more than the choice of model or grid structure (Russell 1997), although design features also matter (for example, see Jang et al. 1995).

Emissions from major industrial stacks are reasonably well known, with continuous monitors providing real time emissions measurements for major facilities. This is not the case for emission from residential, commercial, mobile, and biogenic sources. Industrial 
emissions from sources other than smokestacks (e.g., leaky pipes and valves) are not known accurately. Traditionally, motor vehicle and biogenic VOC emissions have been under and over estimated, respectively (for example, see Marr et al. 2002a and Marr 2002b). Russell (1997) discusses how emissions estimates differ from actual values, observing:

- Motor vehicle VOC exhaust emissions are underestimated by a factor of 2 to 4 (based on Pierson et al. 1990; see also Harley et al. 1997; Sawyer et al. 2000; Marr et al. 2002a).

- Biogenic VOC emissions are uncertain by a factor of 3 or more (based on Geron et al. 1994; Simpson et al. 1995).

- Other VOC sources, if studied in more detail, would be found to be very uncertain too.

- Mobile $\mathrm{NO}_{\mathrm{x}}$ emissions are better understood than mobile VOC emissions (based on Pierson 1990; see also Sawyer et al. 2000).

- Biogenic $\mathrm{NO}_{\mathrm{x}}$ emissions estimates are still being developed, and may be important in some areas (see Matson 1997).

Adding to emissions uncertainties is the need for their temporal and spatial specification. In one recent study, a fuel-based estimate of diesel truck emissions for the San Francisco Bay and San Joaquin Valley air basins found that emissions decrease 7080 percent on weekends (Marr et al. 2002a). Emissions variation with meteorology is quite complicated, as changes in photolytic flux, temperature and moisture can influence emissions (Lamanna and Goldstein 1999; Schade et al. 1999). Efforts to distinguish weekday and weekend emissions budgets are ongoing but hindered by limited knowledge of day-to-day changes in anthropogenic activity. 
Emissions estimates typically have less spatial resolution than needed for a gridded modeling application (Russell 1997; Sawyer et al. 2000). All emissions, except some major point sources, are treated as area sources emitted at the surface because they are summed within grid cells and assumed to mix instantaneously in the cell. This may cause an important distortion in the chemistry in the case of large point sources ${ }^{54}$ (Russell 1997). Plume-in-grid modeling does allow for simulation of sub-grid, point source emissions, where the emissions plume is assigned to the appropriate horizontal layer based on consideration of plume rise and meteorology. Overlapping plumes are not treated explicitly in such plume-in-grid models.

Biogenic emissions estimates rely on knowledge of the surface area coverage of plant types, indices of leaf mass per plant type, and emissions rates per unit leaf mass per plant type. This is an active field of research because emissions rates are characterized incompletely for the myriad plant types and VOC species emitted.

Important assumptions used to estimate future emissions pertain to the rates used for population and economic growth and for land use conversion, forecasted changes in driving patterns, and the anticipated effectiveness and rates of implementation of emissions control technology. Inevitably, these assumptions will lead to some error. None of these assumptions can account for unanticipated gradual changes, such as the rise in popularity of sport utility vehicles and light-duty trucks during the 1990's, or abrupt changes, such as a sudden increase in crude oil prices that leads power producers to switch from oil to natural gas fuel.

\footnotetext{
${ }^{54}$ Assuming instantaneous pollutant mixing within grid cells has the effect of slowing down the chemistry at the source, where concentrations will be higher in reality, and speeding it up at distant parts of the grid,
} 


\section{Observational Data}

Observational data collected to initialize the modeling system, provide boundary conditions, and evaluate model performance have uncertainties due to limited characterization of their spatial and temporal variability. Observational data also have uncertainties due to limitations of the monitoring equipment, user error, and monitoring network design. Some pollutant species are easier to measure than others. For example, measurements of $\mathrm{NO}_{\mathrm{x}}$ may actually capture $\mathrm{NO}_{\mathrm{y}}$, which includes $\mathrm{NO}_{\mathrm{x}}$ plus products of $\mathrm{NO}_{\mathrm{x}}$ oxidation. ${ }^{55}$ Equipment may malfunction or may not be properly calibrated. The determination of concentrations resulting from sampling into canisters may have errors due to a flawed analytical technique.

Monitor location can affect measurement bias. Routine monitoring stations are often located near sensitive receptors (e.g., population centers) or to emphasize some emissions sources over others. For example, stations may be sited near roadways to observe carbon monoxide hot spots. Measurements of ozone taken near roadways are underestimates of larger spatial scale concentrations due to local scavenging by vehicular nitrogen oxides emissions. Routine monitoring rarely characterizes ambient conditions aloft with sufficient spatial resolution, even though such observations are needed to initialize multilayer models.

Another concern is incommensurability between the spatial scales of estimated and observed pollutant concentrations. Measurements taken at one or several specific points within a grid cell typically represent volumes on the order of tens of cubic meters.

where concentrations would be lower in reality.

${ }^{55} \mathrm{NO}_{y}$ may consist of $\mathrm{HNO}_{3}, \mathrm{HONO}, \mathrm{N}_{2} \mathrm{O}_{5}, \mathrm{NO}_{3}$, peroxyaceytl nitrates (PAN), organic nitrates, particulate nitrates, and any other reactive nitrogen compounds present (Seinfeld and Pandis 1998). 
Modeled concentrations are grid-cell averages for volumes ranging up to several hundred cubic kilometers (NARSTO 2000b). ${ }^{56}$

\section{Meteorology}

The meteorological model relies on observations typically lacking in the spatial and temporal detail needed to initialize meteorological fields. Interpolation using sparse observational data can lead to errors in calculated meteorological fields. The state-of-thescience has progressed from objective interpolation to prognostic methods based on solution of fluid dynamics equations. Errors associated with initial and boundary conditions and with numerical solution methods can amplify temporally (Russell and Dennis 2000). The application of Four Dimensional Data Assimilation dampens the temporal growth in errors by causing model results to conform to observations at regular intervals (Stauffer and Seaman 1990). Specifically, the three components of wind velocity calculated as a function of time are nudged toward measured values. Doing so, however, reduces the amount of observational data remaining for performance evaluation.

An important characteristic of meteorology is solar radiation, which influences temperature, photochemical reactions, and vertical mixing (Russell 1997; Vuilleumier et al. 2000; Vuilleumier et al. 2001). Radiative transfer depends on incoming solar radiation, scattering and absorption by gases, aerosols and ground-level surfaces. Surface albedo influences actinic flux, which must be known to estimate the chemical processing. The effects of aerosols on radiative transfer, both direct and indirect (i.e., due to clouds),

\footnotetext{
${ }^{56}$ Surface layer grid cells range from 4 to $40 \mathrm{~km}$ in the horizontal and 50 to $200 \mathrm{~m}$ in height, which equals a volume range from less than 1 to $320 \mathrm{~km}^{3}$.
} 
are major sources of uncertainty. Actinic flux estimation requires description of aerosols spatially and temporally; observations available to do so accurately are only recently available. There remain large uncertainties in aerosol concentrations, composition, and optical properties. Representations of key cloud chemistry and physics processes and properties within air quality models are also limited. With the exception of $\mathrm{NO}_{2}$, atmospheric gases that affect radiative transfer are better characterized and there are fewer uncertainties associated with their contributions.

\section{Chemistry}

Atmospheric chemistry is known incompletely, as it involves hundreds of pollutant species and thousands of reactions. Reaction rates and pathways are understood adequately for less than one quarter of the chemical species observed in the atmosphere (NARSTO 2000b). Even if known completely, atmospheric chemistry cannot be represented in its entirety because it would impose excessive computational demand. Fortunately, only a subset of essential reactions need be represented. Several approaches are used to simplify the chemistry, among these are the steady state approximation for radical species, and the use of structural and functional lumping for organic species (Lurmann et al. 1987; Gery et al. 1989). Although they give similar results for ozone, they do not do so for other pollutant species (Jeffries and Tonnesen 1994). Uncertainties associated with atmospheric organic chemistry are more significant when ozone formation is limited by the availability of organic compounds; that is, when the ratio of VOCs to $\mathrm{NO}_{\mathrm{x}}$ is small. Chemical kinetic parameters are determined experimentally, so the values are subject to experimental errors. 


\section{Resolution}

Representing the range of scales relevant to the physical system places great demands on a PAQSM (Dennis et al. 1996). Models must span orders of magnitude in time and space, even though outputs are usually sought for time periods covering hours or days. ${ }^{57}$ A compromise must be met between the inherent resolution of the processes of interest and scales imposed to manage the limitations of available information and computational intensity.

The spatial and temporal resolution of the inputs from the meteorological and emissions models are determined by modeling system specifications, such as grid structure (i.e., horizontal and vertical resolution). Emissions estimates and meteorological field inputs must be resolved to these scales. Furthermore, emission descriptions must be compatible with the speciation requirements of the chemical mechanism.

Chemical and meteorological measurements are often not available at desired temporal or spatial resolution. No matter what grid-size is selected for use, processes occurring at sub-grid spatial scales are represented average values used for grid cells. The significance of this limitation is still being explored (NARSTO 2000b).

There is a trade-off between grid structure resolution and computational intensity. Finer grids, both horizontally and vertically, reduce errors associated with numerical solution techniques, better represent point-oriented observations, and facilitate the approximation of physical processes, such as wind shear and vertical mixing. Data availability becomes limiting since finer resolution is not helpful when inputs, such as observations and estimates of emissions and meteorological variables, are not similarly 
resolved. New approaches, like nested-grid refinements (Morris et al. 1991; Odman and Russell 1991; Byun et al. 1995), might address this tradeoff by providing finer spatial resolution for emissions "hot spots".

\section{Uncertainties in Model Formulation}

Uncertainties associated with model formulation may be due to erroneous or incomplete representations, incommensurability, numerical solution techniques, and choice of modeling domain and grid structure. Simplified representations are necessary when knowledge is incomplete, or when more thorough or precise specification would increase computational intensity excessively. Where more than one algorithm is available and appropriate, choosing one inevitably means accepting some uncertainties over others.

\section{Turbulence}

Uncertainties arise from the deterministic representation of turbulent diffusion transport using the gradient transport hypothesis in conjunction with the diffusivity coefficient, Kj. The approach limits the model's applicability in the lower limits of the spatial and temporal scales (Russell 1997). The validity of the ADE is predicated on two assumptions. Atmospheric turbulence is assumed stationary for the averaging time period of interest ( $\sim 30$ to 60 minutes for most applications). Also, the characteristic temporal and spatial scales in the gradients of the turbulent velocity correlations are assumed large compared with the time resolution and average distance that a fluid particle travels in that time period. These assumptions break down at small spatial and temporal scales.

\footnotetext{
57 The averaging times for ozone NAAQS concentration thresholds are one and eight hours.
} 


\section{Removal Processes}

Uncertainties in estimating pollutant removal are associated with the treatment of pollutant transport near surfaces and the net flux of pollutants from various types of vegetation and soils. Deposition is the pathway by which pollutants are removed from the atmosphere via the physical transport to the surface and the physical/chemical interactions occurring there. The nature of these interactions for various species and surface types is a source of uncertainty. In wet conditions, deposition involves washout of pollutants with precipitation. Dry deposition involves no atmospheric hydrometeors (i.e., cloud and fog droplets, rain, snow).

Available measurements of deposition are limited (Wesely and Hicks 2000). Studies of the processes that control dry deposition require direct measurements of the air-tosurface exchange. Micrometeorological approaches used to characterize deposition, like eddy correlation and gradients, are not well developed for non-uniform landscapes, such as hilly terrain, or for reactive pollutants, such as $\mathrm{NO}_{2}$. The ability to parameterize the processes affecting dry deposition and re-emission is limited by the complexity and variability of the chemical, physical, and biological characteristics of the surfaces and the diversity of pollutants and surface types.

Wet deposition and other aqueous phase physical and chemical interactions are among the most complex atmospheric processes to model. Indicative of the challenge are the range of scales (i.e., $10^{-6} \mathrm{~m}$ to $10^{6} \mathrm{~m}$ ) at which relevant processes occur, the multiple phases (i.e., gas, liquid, aerosol) and states of aqueous phases (e.g., cloud droplets, fog, rain, snow, ice), and different processes occurring within and below clouds (Seinfeld and Pandis 1998). For simulations involving short-term and urban-scale ozone episodes in 
areas of low humidity (e.g., Western U.S. ), wet deposition is not important because high ozone typically occurs on dry days. However, in humid areas (e.g., Midwestern and Eastern U.S.) and for regional-scale, seasonal-length modeling, precipitation scavenging and cloud dynamics become significant. These processes are treated using a washout parameter determined empirically or by calculating rates of pollutant diffusion into water droplets. Simulation is hindered by deficient or inaccurate knowledge of the size distributions of water droplets and ice crystals, as well as incomplete understanding of cloud dynamics. Consequently, wet deposition is one of the more uncertain outputs of meteorological models (Seaman 2000).

Another removal process is the entrainment of pollutants aloft (i.e., above the mixing layer). As modeled ozone episodes may last many days, overnight storage and subsequent reintroduction of aloft pollutants requires description of the rate of vertical mixing. Doing so introduces uncertainty because knowledge of turbulent flow, vertical exchange, and pollutant concentrations at the top of the modeling domain is usually limited.

\section{Aerosols}

Historically, models sometimes treated the transport of aerosols, but never their physical and chemical processing. Gas and liquid phase chemistry requires specification, as does the chemistry involving pollutant reactions with aerosol and water droplet surfaces. This is especially so when simulating regional spatial scales and entire ozone seasons. Cloud droplets act as small reactors, influence pollutant mixing, compete with gas phase chemistry, and affect rates of wet deposition. Knowledge of heterogeneous (multi-phase) reactions is severely deficient. Treating cloud processes is computationally intensive and requires input data that are rarely available. Complete treatment requires 
characterization of the size distributions of aerosols, as well as their other chemical and physical properties. Consequently, there is considerable uncertainty associated with the treatment of cloud dynamics by deterministic meteorological models. Typifying how uncertainties are interdependent, those associated with cloud predictions exacerbate uncertainties in biogenic emission estimates that are sensitive to photolytic flux.

\section{Numerical Solution}

Solution techniques pertain to the numerical methods used to solve the set of coupled differential equations that cannot be solved analytically. Each of these conserves mass approximately (Russell 1997). Solution-related errors tend to resemble artificial dispersion, thereby spreading would-be concentration peaks spatially. Nonetheless, solution techniques are believed to contribute a small amount of error to model predictions relative to errors associated with emissions estimates, representation of meteorology, and values used for boundary conditions (Dabdub and Seinfeld 1995).

\section{Variability}

Variability refers to stochastic atmospheric and anthropogenic processes. It contributes to uncertainties discussed previously, notably those associated with emissions estimates and representations of chemistry and meteorology. Here, its contribution to uncertainty is discussed in two respects: the implications of using means to represent values that vary and the inability to treat inherent variability.

The deterministic treatment of stochastic processes using nominal mean values is a source of uncertainty. For example, real motor vehicle driving activity and associated emissions vary daily, hourly, monthly, etc. Attempts to estimate vehicular emissions 
introduces uncertainty associated with the choice of representation. Although it is desirable to generate separate estimates for weekdays and weekends, knowledge may not be adequate to do so. Furthermore, when mean values are used, simulation of extreme realizations is prohibited (NARSTO 2000b).

Although it may be possible to represent stochastic processes using probabilistic methods, doing so does not eliminate the uncertainty inherent to variability. The estimate of vehicular emissions does not associate weather conditions and driving, so feedback effects are not represented. Extreme events are not represented either, such as changes in emissions from congestion caused by a traffic accident.

With few exceptions (for example, see Vuilleumier et al. 2000), neither the modeling systems nor the air quality planning efforts using modeling tools have incorporated representations of variability. At the modeling system level, emissions and meteorology are not characterized probabilistically. At the planning level, the few simulated ozone episodes may not represent the myriad conditions capable of causing violations of ozone air quality standards. With increases in computing capabilities and expanded observational databases, efforts are now under way to model full ozone seasons (for examples, see Winner and Cass 1999; Winner and Cass 2001), presumably capturing numerous ozone episodes to address concerns about meteorological and emissions variability. It may be necessary to model several ozone seasons to assess fully the range of variability and to evaluate air quality on days when ozone violates the 8-hour concentration threshold in addition to the 1-hour threshold. Doing so, however, may involve trade-offs. Although variability may be better represented, each modeled episode 
may be less accurate, since they will receive less detailed attention and be based on routine rather than intensive observations.

\section{Uncertainties in Use of Modeling Results}

Although not strictly within the scope of this review, a fourth uncertainty relates to the use of PAQSM results. Decision-makers must decide what to do with model output, including weighing it against other information. PAQSM output may be not compatible with the decision-makers' needs. Consequently, there is uncertainty about how PAQSM output will be incorporated into decisions.

Another aspect of uncertainty arising from the use of model outputs is the characterization and incorporation of uncertainty. To date, formal model evaluation efforts have been inadequate (Russell 1997; NARSTO 2000b; NRC 1991; Dennis et al. 1996). Air quality planning oversight agencies provide limited guidance for treating uncertainty. Consequently, there is uncertainty about the nature of PAQSM output uncertainties, as well as how policy-makers manage the limited knowledge they do have about uncertainties.

Finally, the possibility of uncertainties that are not yet known must be acknowledged. Unknown unknowns have frustrated past modeling and planning, and may continue to do so, as exemplified by the continual discovery of new sources of emissions.

\section{F. Experiences With PAQSM Uncertainties}

Experiences with models and their uncertainties indicate the potential benefits of rigorous uncertainty assessment. In one example of the uncertainties associated with model inputs, Hansen (2000) queried experts to estimate the uncertainties of 128 key 
input variables of PAQSM. The experts described 95 percent confidence intervals. The modeling system was used by the Ozone Transport Assessment Group (OTAG) to evaluate emissions control needs to bring the northeastern U.S. into attainment with the ozone NAAQS. The effort was concerned with long-range emissions transport from Midwestern states (OTAG 1997). Table III-A shows findings of Hansen (2000).

\section{Table III-A: Experts’ Estimates of Model Input Uncertainties (Hansen 2000, Table 3-1)}

\begin{tabular}{|c|c|c|c|}
\hline $\begin{array}{l}\text { PAQSM Input } \\
\text { Category }\end{array}$ & Input Variable & $\begin{array}{l}\text { Uncertainty } \\
\text { Range }\end{array}$ & $\begin{array}{c}\text { Standard Deviation } \\
\text { (log-normal distribution } \\
\text { unless noted) }\end{array}$ \\
\hline \multirow{2}{*}{$\begin{array}{l}\text { Initial } \\
\text { Conditions }\end{array}$} & $\mathrm{O}_{3}$ Concentration & Factor of 3 & 0.549 \\
\hline & $\mathrm{NO}_{\mathrm{x}}$ or VOCs Concentration & Factor of 5 & 0.805 \\
\hline \multirow{2}{*}{$\begin{array}{l}\text { Boundary } \\
\text { Conditions }\end{array}$} & $\mathrm{O}_{3}$ Concentration Aloft or at Side & Factor of 1.5 & 0.203 \\
\hline & $\begin{array}{l}\mathrm{NO}_{\mathrm{x}} \text { or VOCs Concentration Aloft } \\
\text { or at Side }\end{array}$ & Factor of 3 & .0549 \\
\hline \multirow[t]{9}{*}{ Meteorology } & Wind Speed & Factor of 1.5 & 0.203 \\
\hline & Wind Direction & +/- 40 degrees & 20.0 (normal) \\
\hline & Air Temperature & +/- 3 Kelvins & 1.5 (normal) \\
\hline & Relative Humidity & 30 percent & 15.0 (normal) \\
\hline & $\begin{array}{l}\text { Daytime Vertical Diffusivity } \\
\text { below } 1000 \text { meters }\end{array}$ & Factor of 1.3 & 0.131 \\
\hline & $\begin{array}{l}\text { Nighttime Vertical Diffusivity at } \\
\text { all heights }\end{array}$ & Factor of 3 & 0.549 \\
\hline & Rainfall Amount & Factor of 2 & 0.347 \\
\hline & Cloud Cover & 30 percent & 0.15 (normal) \\
\hline & Cloud Liquid Water Content & Factor of 2 & 0.347 \\
\hline \multirow[t]{2}{*}{ Emissions } & Major Point Source $\mathrm{NO}_{\mathrm{x}}$ or VOC & Factor of 1.5 & 0.203 \\
\hline & All other emissions estimates ${ }^{58}$ & Factor of 2 & 0.347 \\
\hline Photolysis Rates & Six reactions & Factor of 2 & 0.347 \\
\hline $\begin{array}{l}\text { Carbon Bond IV } \\
\text { Reactions }\end{array}$ & Reactions 1 thru 83 & $\begin{array}{l}\text { Factors ranging } \\
\text { from } 1.17 \text { to } 2.5\end{array}$ & $0.079-0.458$ \\
\hline
\end{tabular}

${ }^{58}$ Includes $\mathrm{NO}_{\mathrm{x}}$ and VOC emissions estimated for biogenic, mobile, and area sources. 
Although indicative of the range of input uncertainties, the study of OTAG modeling is not representative of modeling carried out elsewhere. A more comprehensive evaluation of modeling was carried out as part of NARSTO. Roth et al. (1997) evaluated eleven urban-scale and four regional-scale modeling applications using 20 criteria describing the soundness of the model formulation, representativeness of the modeled episode(s), adequacy of the input database and emissions estimates, and the sufficiency of performance evaluation, peer review and documentation. The results of the review are summarized in Table III-B.

Roth et al. (1997) found that modeling limitations were extensive and ubiquitous. The difference between observed and modeled one-hour ozone concentrations ranged from 20-35 percent. Although the regional studies tended to be more satisfactory than urban-scale studies, most studies were found to have some or major deficiencies and omissions. Furthermore, no study performed an adequate or satisfactory estimate of modeling uncertainties. 
Table III-B: Modeling Studies Grades (Roth et al. 1997)

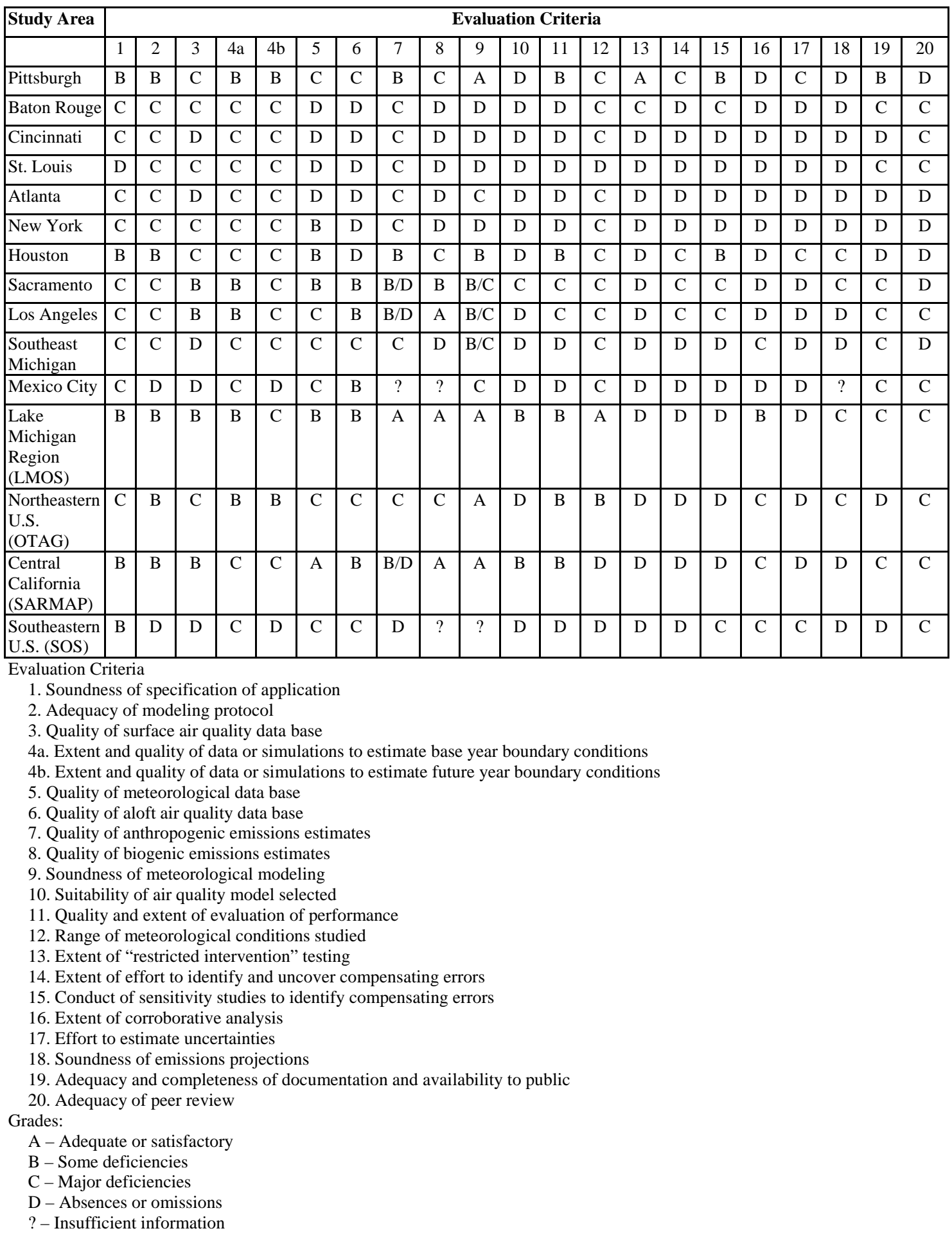


The work by Hanna, Roth, and colleagues indicate a commitment to acknowledge and evaluate uncertainties in PAQSM in the research realm. Although the findings of Roth et al. (1997) indicate less interest in uncertainty for regulatory applications, such efforts certainly benefit from progress made by researchers. In addition to improvements noted above pertaining to meteorological and plume-in-grid modeling, the NARSTO Assessment (2000b) highlights several modeling advancements during the 1990's, including:

- Variable grid-size nesting to permit a range of spatial resolution within the modeling domain.

- Improved treatment of biogenic emissions, including isoprene estimate accuracy and incorporation of associated pollutant species into chemistry representations.

- Progress toward multi-pollutant modeling, notably the development of algorithms to represent aerosol dynamics.

Furthermore, the NARSTO Assessment acknowledges improved approaches for conducting sensitivity analyses and for estimating error distributions of model outputs. These approaches are reviewed below after the suite of methods available to evaluate uncertainties in modeling is introduced.

\section{G. Framework for Uncertainty Analyses}

Having identified uncertainties associated with PAQSM, the methods for evaluating uncertainties are introduced in this section. Sensitivity and uncertainty analyses are defined. Performance evaluation required for models applied in a regulatory context are described and evaluated. The suit of available uncertainty analyses methods is 
introduced. Last, two questions are presented to motivate and focus the synthesis of the disparate information yielded by uncertainty evaluation methods.

\section{Uncertainty Analyses Defined}

Assessing uncertainties in modeling involves two approaches typically referred to as sensitivity and uncertainty analyses. Sensitivity analyses estimate the response of model output to changes in inputs or model formulations. They help modelers assess the relative importance of inputs or formulations, and their uncertainties, on uncertainties in outputs.

Sensitivity studies should be preceded by a determination that the relationships and, therefore, the sensitivities depicted by the union of mathematical formulas and input data provides an adequately reliable analog of the phenomena of interest. Adequacy needs to be defined according to the decision-making context. In this respect, sensitivity analyses are one component of the broader framework defined herein as uncertainty analyses.

When sensitivity studies are used for the latter purpose - to describe the uncertainties in model output due to input uncertainties - it is sensitivity/uncertainty analysis.

Uncertainty analysis is defined by Morgan and Henrion (1990, page 39) as:

The computation of the total uncertainty induced in the output by quantified uncertainty in the inputs and model and the attributes of the relative importance of the input uncertainties in terms of their contributions.

The framework presented below provides the information per the above definitions. It also examines performance through intermediate products (e.g., meteorological fields) and includes using data and models exogenous to the PAQSM. Products of uncertainty evaluation may be qualitative or quantitative, including: 
- Quantify model sensitivities, notably the dependence of outputs on local changes in inputs, formulations, and design features, such as grid resolution

- Provide information to make probabilistic statements about the indications of model output, notably the likelihood that future air quality estimated by the model will be realized

- Increase confidence that the model is sufficiently valid for the decisionmaking need

- Identify and assess the significance of compensating errors

Before introducing the components of a comprehensive model uncertainty analysis, current regulatory requirements for performance evaluation are summarized to make a case for more extensive uncertainty assessment.

\section{Operational Performance Requirements}

State and federal agencies establish formal processes for model validation, verification and application. The EPA promulgated guidelines on which PAQSMs to use and how to apply and evaluate them (EPA 1992a; 1993b, 1996a, 1996b) ${ }^{59}$. In California, CARB provided similar guidance (CARB 1992). EPA and CARB requirements constitute an "operational analysis" that relies on comparison of estimated and observed peak ozone, expressed in terms of bias and error metrics. ${ }^{60}$ Although necessary measures, these metrics are not sufficient indicators of reliable model performance. They do not address the concern that models may appear accurate for the wrong reasons. Errors that offset each other (“compensating errors”) may indicate, incorrectly, adequate model

\footnotetext{
${ }^{59}$ Additional guidance is provided for development and use of input data, notably emissions estimates (for examples, see EPA 1993a; 1997b; 1999c).

${ }^{60}$ Here, error and bias are defined strictly. Error is the mean of the absolute values of the differences between the computed (i.e., model estimated) and observed values; bias is simply the mean of the differences. Minimum performance criteria for PAQSM used in State Implementation Plans are that peak ozone predictions have paired bias and error normalized for peak ozone of less than 15 and 35 percent, respectively, and a bias unpaired in space and time of less than 20 percent (CARB 1992).
} 
performance. The risk of having such errors present is the development and adoption of ineffective or counterproductive emissions control strategies.

Biases or compensating errors are often hard to detect unless they are sought. The NARSTO Assessment (2000b) offers two examples of compensating errors. In Kern County, California, two studies using the same model and input data produced conflicting conclusions about the relative effectiveness of pursuing $\mathrm{NO}_{\mathrm{x}}$ or VOC control strategies, due to different assumptions about VOCs concentrations aloft and meteorological conditions in areas without observations (NARSTO 2000b). In another example, a modeling study in New York state yielded considerable difference in ozone estimates depending on the method used to generate wind fields and mixing heights (Sistla et al. 1996).

Performance criteria measured in terms of error and bias metrics appear to be based largely on experiences with model performance (NARSTO 2000b). More relevant criteria would relate to what is meant to be learned by the modeling effort. For example, the needed accuracy in estimates of observed peak ozone concentrations might be determined by the amount of ozone reduction necessary to meet the standard.

Criteria based on past performance may lead to an approved model incapable of estimating future ozone with adequate reliability. Ozone control plans based on such models may be similarly unreliable. This concern is exacerbated by lower absolute values being modeled as peak observed ozone declines and the new 8-hour ozone standard has a lower absolute concentration threshold. 
Statistical measures tell a partial story, one that is quite helpful when there is a "big problem" associated with performance, but less so (from the standpoint of uncertainty estimation) when there is not. Concerning limitations of operational evaluations to estimate uncertainty, one must consider that:

- Uncertainties associated with both observed and modeled concentrations should be taken into account.

- Some measurements do not compare exactly with the quantity modeled. Per the discussion of incommensurability above, observed and modeled concentrations represent different spatial averaging characteristics. In another example that also indicates uncertainties in measurements, what is often considered observed $\mathrm{NO}_{2}$ will likely contain some peroxyactyl nitrate (PAN) and $\mathrm{HNO}_{3}$ as well.

- The error and bias metrics used to summarize model performance indicate how well the model simulates the observed conditions, but do not account for the possibility that the observed value may not be the true value of interest (e.g., peak ozone observed may not be the peak occurrence).

- Estimates of models may not be "best estimates" and their expected bias should be considered. For example, ozone concentrations observed near roadways should be depressed relative to a model-estimated value due to reaction of ozone with NO. A less biased comparison would be made between observed and modeled "ozone $+\mathrm{NO}_{2}$ ".

An unbiased, integrated estimate of uncertainty must take into account these additional factors. The NARSTO (2000a) and NRC (1991) assessments call for model uncertainty evaluations that extend beyond the criteria currently in force. Other researchers note that the practice of PAQSM performance analysis is wanting (Dennis et al. 1996; Roth et al. 1997; Russell 1997; Roth 1999; Russell and Dennis 2000).

In 1999, the EPA issued draft guidance on how to interpret modeling and other analyses to demonstration attainment of the 8-hour ozone NAAQS (EPA 1999b). It lists 
seven approaches to performance evaluation, listed below, that will address the need for identification of compensating errors and other uncertainty concerns:

1. "Big picture" graphical assessment by comparing

2. “Ozone metrics” statistical comparisons per operational evaluation

3. Compare observed and predicted precursor and species comparisons

4. Compare predicted source attribution factors with estimates obtained using observational models

5. Compare observed and predicted on weekends versus weekdays

6. Compare observed and predicted ratios of precursor species

7. Compare retrospectively model predictions with observed trends

The guidance also addresses concerns that the modeled episode may not be the peak occurrence (using a "relative reduction factor") and recommends modeling more ozone episodes than previous practice. It de-emphasizes modeling, somewhat, by allowing for a "weight of evidence determination" uses that available air quality, meteorological, and emissions data to complement a modeling analysis, and to develop a conceptual description of an attainment problem. ${ }^{61}$ Although the document is not yet finalized (Baldridge 2002), it represents a courageous exploration of model uncertainty, and a detailed discussion of diagnostic analyses.

\section{Framework for Developing Uncertainty Information}

Although CARB and EPA standardized statistics for evaluating the adequacy of PAQSM performance, no comprehensive approach exists for estimating uncertainties in the results or outcomes of modeling. Oftentimes uncertainties simply are not estimated. When they are, a necessarily limited approach is taken, such as the operational analyses

\footnotetext{
${ }^{61}$ The FCAA language establishing the "attainment demonstration" in SIPs (see excerpt in Appendix B discussion of Modeling Requirements for Ozone Attainment Demonstration Plan) requires the use of models or "any other analytical method determined...to be at least as effective," which includes a weight-
} 
described in the previous section. A typical uncertainty assessment will generally address one (or more) aspect(s) of uncertainty, but will not provide a complete or encompassing estimate (see Morgan and Henrion 1990). The modeling community simply does not know how to do this (NARSTO 2000b).

The available approaches for developing uncertainty information may, when combined, provide the information necessary to comprehensively assess modeling uncertainties. Figure III-4 is a schematic diagram that brings together the several limited approaches now in use. (Again, no such comprehensive, though incomplete, methodology has yet been applied in a modeling study.) It shows how they might interact or link. These methods are identified in this section, and explicated in later chapters.

The individual, limited approaches represented in the diagram are the following:

- Operational evaluation involving calculation of standard performance statistics

- Diagnostic analyses conducted typically to identify ways to improve operational performance

- Sensitivity and sensitivity/uncertainty analyses

- Alternative base case (ABC) analysis

- Subjective/judgment methods

- Corroborative methods 
Figure III-4: Air Quality Model Uncertainty: Evaluation and Analyses

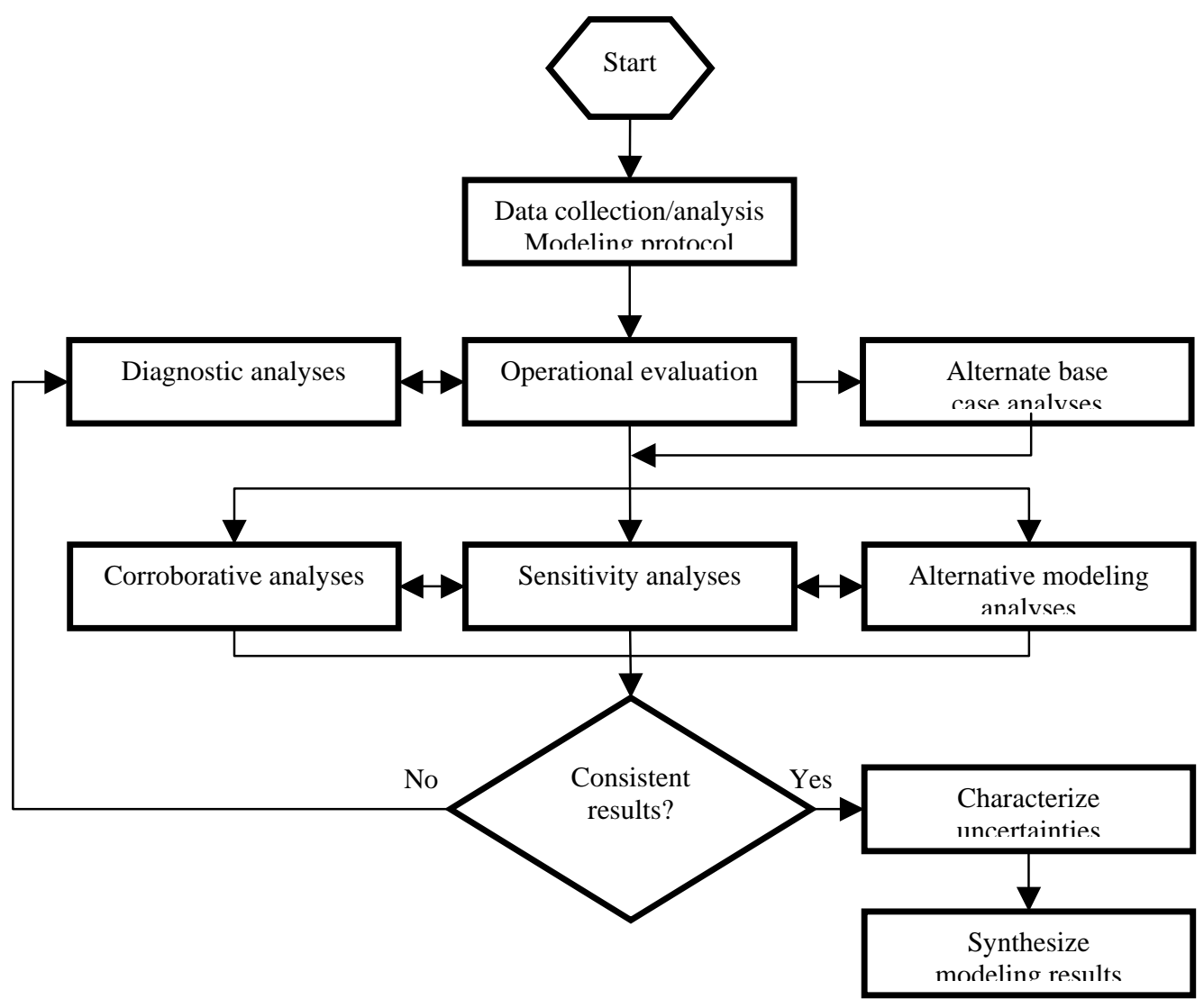

The two dominant approaches - sensitivity and diagnostic analyses - are reviewed at length in the following chapters. Alternative and corroborative modeling and subjective methods are also reviewed. However, given the history of focus on sensitivity and diagnostic assessment, experiences with alternative, corroborative and subjective methods are sparse, so their potential is more speculative.

Assessing and estimating PAQSM uncertainties involves exercising the entire PAQSM or components of it. Modeling results may be corroborated through other means too. Diagnostic assessment focuses on evaluating the performance of individual modules 
of the PAQSM. Corroborative and alternative modeling methods include model-tomodel comparisons, corroboration using different types of models (e.g., EPA’s Mapper) and data analyses, and the solicitation of experts' opinions. Uncertainty analyses also may incorporate alternative scenarios of, for example, emissions estimates simulated.

Generally speaking, once the results of a given approach emerge, it is usually difficult to engage them with other sets of results. Methods of integration and synthesis have not been developed. It may be that they are unlikely to be developed. Synthesis occurs instead through the judgment of the investigator: the investigator pursues subjective integration only when he or she feels that it is necessary and there is no alternative.

Though no synthesis has yet been attempted, nor have all of the components of the framework been used at one time to evaluate the uncertainties of a modeling exercise applied for planning, it is possible to speculate about the content of the uncertainty information to be generated. As these analyses are recommended to aid decision-makers, it is their needs that must define uncertainty analyses products.

The question facing decision-makers developing a plan to meet the federal ozone NAAQS is: How much must current and anticipated future $\mathrm{NO}_{x}$ and VOC emissions be reduced to meet the ozone NAAQS by a specified deadline? When the control decision is based on model results, the question necessitated is: What is the likelihood that ozone NAAQS will be met when the model indicates that planned emissions reductions will yield attainment? The answer to the latter question is a probabilistic statement, and indicates the need to synthesize uncertainty information probabilistically. The most common forms of such information are confidence intervals or expected values with error bars. 


\section{H. Conclusions}

Fine et al. (2003) present nine conclusions about efforts to date to evaluate uncertainties in PAQSMs:

1. Uncertainties pervade the use of models. Consequently, a range of model estimates may be anticipated for a given set of inputs and their associated uncertainties. Thus, estimates of uncertainties should be made and factored into processes involving model-based decision-making associated with air quality issues.

2. Methods available today that are truly useful either partially address the estimation need or focus on a defined, limited part of the problem. Unfortunately, no method is now available for estimating uncertainty in modeling that is comprehensive in scope.

3. A comprehensive method for analyzing uncertainty information would (a) propagate uncertainty from each component of the modeling system through the system into an estimate of uncertainty associated with model output, (b) elucidate bias, and (c) account for variability. The method would also synthesize and integrate results from the various methods employed to estimate uncertainty comprehensively. The products of a comprehensive uncertainty assessment would be distributions or probabilistic statements characterizing the uncertainty of model estimates.

4. Developing a comprehensive approach to uncertainty analysis would be very valuable. Its feasibility should be assessed. Such an approach may not be possible, since nothing in the literature suggests so.

5. In practical applications, visual inspection of plots of concentration versus time for pollutant species of interest provides adequate information to determine if model performance is sufficiently acceptable to merit proceeding with comprehensive uncertainty assessment. In many cases, model performance is wanting. Where performance is unacceptable, major flaws in the model should be corrected prior to obtaining uncertainty information.

6. Sensitivity/uncertainty analysis is by far the most frequently used method for estimating uncertainty. Its focus is on the response of a dependent variables to changes in inputs. Where the response is significant, uncertainty is likely to be important; where the response is small, the converse is expected. Sensitivity analysis does not address bias as an element in uncertainty explicitly. Generally, it is not suited for this use, as the main assumption made is that the model is substantially correct in its representation of reality.

7. As the key element of a comprehensive assessment of uncertainty, the development and application of methods for identifying, estimating, and reducing biases (i.e., mitigating or eliminating flaws in model representations) should be made a priority. This includes determining when bias is present, how to identify 
it, and what to do when it corrupts modeling results. Through assessment of bias, model formulation may be improved to increase the probability that the model is performing acceptably well for the right reasons and that modeled sensitivities are reliable. Examining the issue of potential bias typically requires case-specific procedures.

8. In addition to the evaluation of bias, natural and human-induced variability should receive attention in the comprehensive estimation of uncertainty. Some deterministic modeling formulations may simulate well-characterized stochastic processes using statistical sampling techniques, whereas others, such as those used to derive meteorological inputs, are incapable of simulating stochastic processes. The appropriateness and feasibility of developing stochastic models merits attention because they are a potentially attractive means for incorporating variability.

9. Designing a comprehensive approach to uncertainty assessment that can be implemented and that addresses bias and variability requires a major research effort. To date, no such effort has been formulated, let alone undertaken. Rather, the focus has been on portions of the problem, in the absence of a more encompassing plan that might foster a more integrated research program design.

Building upon the above findings are the following:

- There is a commitment to uncertainty assessment in the research arena, but a lack of commitment to assess it comprehensively in the regulatory arena. Performance requirements are inadequate to identify bias or to generate a complete statement about the uncertainties in model results.

- Uncertainty assessment capabilities are currently insufficient to provide to policy-makers complete, synthesized, useful uncertainty information

- It is feasible to develop available methods further, thereby gaining experience and improving capabilities. Doing so requires a commitment to developing and maintaining an adequate observational database. Currently, commitment is lacking to support such an effort. However, progress is being made. There is anticipated regulatory guidance from EPA on how to model to demonstrate attainment of the 8-hour standard. Initial drafts suggest it will be a promising step forward. Observational databases are being improved by ongoing field studies, such as the Central California Ozone Study and the California Regional Particulate Air Quality Study. This new data will be useful for informing the modeling simulation, as well as for evaluating its performance. 


\section{CENTRAL CALIFORNIA AIR QUALITY}

\section{A. Introduction}

This chapter air quality conditions in Central California, focusing on two air pollutants of primary concern in Central California: tropospheric ozone and particulate matter. Discussion starts with the challenge of interpreting observational data. Air quality conditions and trends are described, as are sources of pollutant emissions and studies intended to improve understanding of air quality in Central California.

\section{B. Conditions}

1. Understanding the Data

Even when focusing on a single air pollutant, there are many ways to describe its occurrence. Tropospheric ozone may be described in terms of:

- Magnitudes of peak concentrations

- Location of peak or high concentrations

- Spatial and temporal extent of peak or high concentrations

- Regularity of peak or high concentrations

- Trends over both space and time

The form of air quality standards determines partly the way ambient conditions are described. The NAAQS for particulate matter 10 microns in diameter or small $\left(\mathrm{PM}_{10}\right)$ includes both annual mean and 24-hour average concentrations. The ozone NAAQS is a one- or eight-hour average concentration that cannot be exceeded more than an average 
of once per year. For more description of the standards, refer to New Standards for Ozone and Particulate Matter in Appendix B.

\section{Ozone Conditions in the SJV and Bay Area}

The many ways air quality can be characterized facilitates the telling of different stories about air quality and associated trends. For example, at a public hearing for their 01 Ozone Plan, the BAAQMD presented a chart titled "Bay Area Ozone Trends" showing data from 1965 to 2000. It showed, quite convincingly, that ozone air quality has improved dramatically due to regulatory efforts. Their graphic is reproduced as Figure IV-1. ${ }^{62}$ In the Fall, 2001, issue of Air Currents, the BAAQMD's newsletter, the tactic was repeated when discussing ozone conditions during the summer of 2001, stating,

In 1999 and 2000, there were three days over the federal 1-hour standard each year. Historically, air quality in the Bay Area has shown steady improvement over the past thirty years. In 1969, the region experienced 65 days over the same federal one-hour ozone standard (BAAQMD 2001b).

Figure IV-1: Days Violating Federal 1-Hour Ozone Standard in the BAAQMD, 1965-2000

\footnotetext{
62 The BAAQMD’s 01 Ozone Plan presented data from only 1990 through 2000 in tabular, not graphical form.
} 


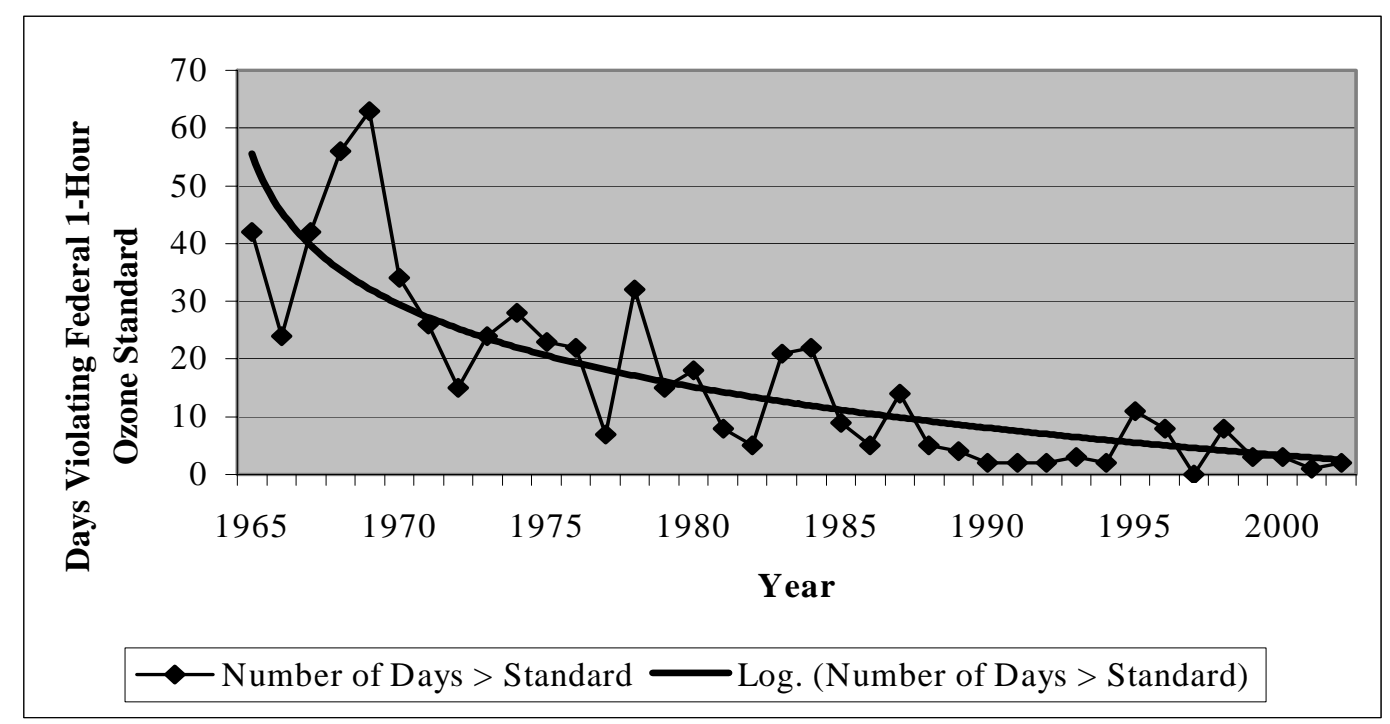

Those in favor of more emissions controls focus on the past decade, as shown in Figure IV-2, which indicates little or no progress during the period. Indeed, the figure showing data back to 1965 suggests the standard is being approached asymptotically. If so, progress may remain difficult to achieve (or detect) for quite some time.

In their 2002 Draft (Draft 02 Ozone Plan) update to the 1994 Ozone Plan, the SJVUAPCD presents ozone trends in terms of the number of days violating the federal 1hour ozone standard from 1980 to 2001. They conclude,

As can be seen, the number of days on which the standard is exceeded is showing a definite downward trend...(SJVUAPCD 2001b, page 1-8). 


\section{Figure IV-2: Days Violating Federal 1-Hour Ozone Standard}

in the BAAQMD, 1990-2002

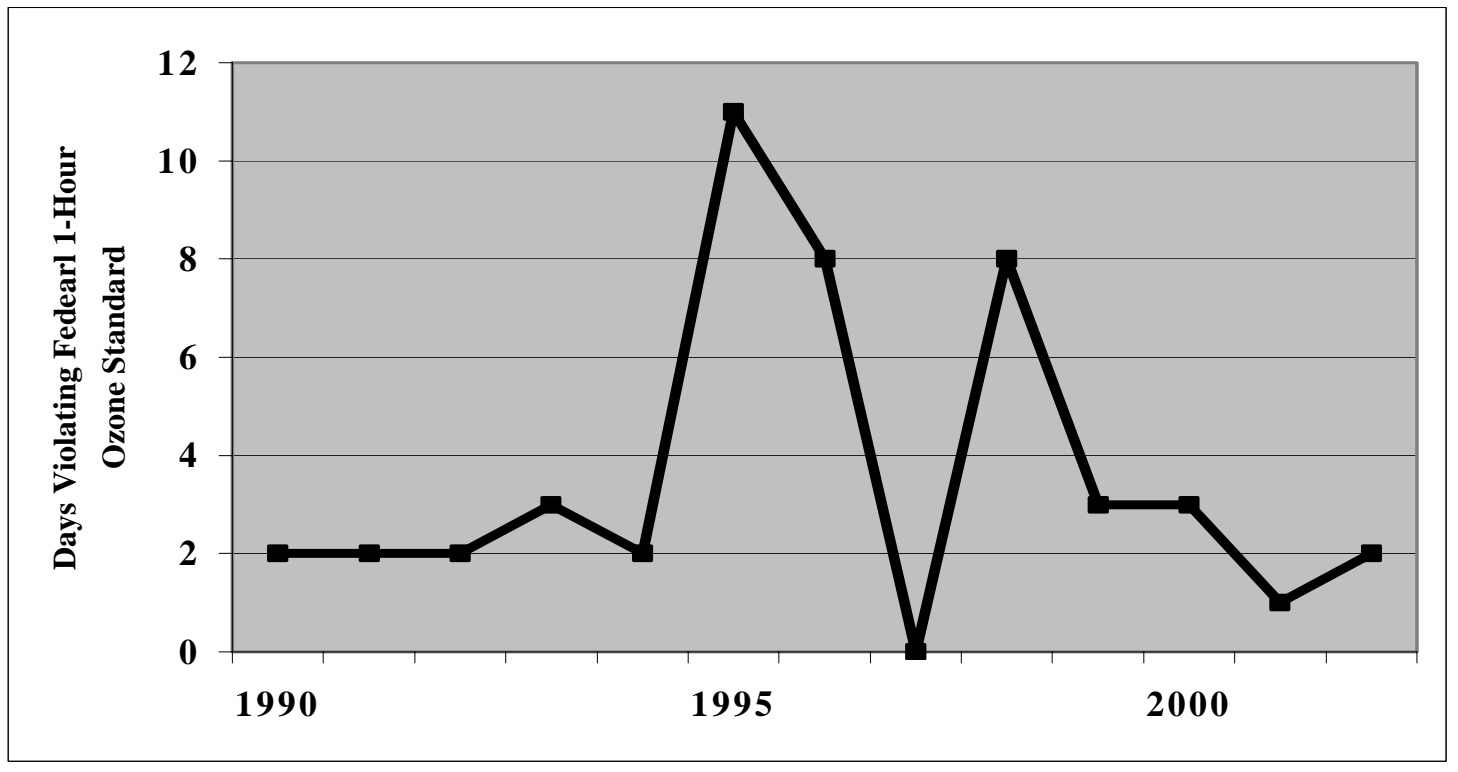

Figures IV-3 and IV-4 show ozone trends in the SJV are similar to those in the Bay Area, except that the situation is far worse. Note the differences in the scales. Looking back to 1980, the SJV appears to be getting cleaner, albeit at a much slower rate than the Bay Area. However, from 1990 to 2000, there is "no significant” downward trend, as indicated in Table IV-B and shown in Figure IV-4.

Conditions in the SJV are worse than in the Bay Area because the State and Federal ozone standards are exceeded on many more days and over a larger area. From 1990 through 2001, the SJV averaged 119 days per year above the state standard, which is nearly one in every three, and 38 days over the less stringent federal standard. Over the same period, the Bay Area exceeded the state and federal standards an average of 19 and 4 days per year, respectively, .

\section{Figure IV-3: Days Violating Federal 1-Hour Ozone Standard} SJVUAPCD, 1980-2002 


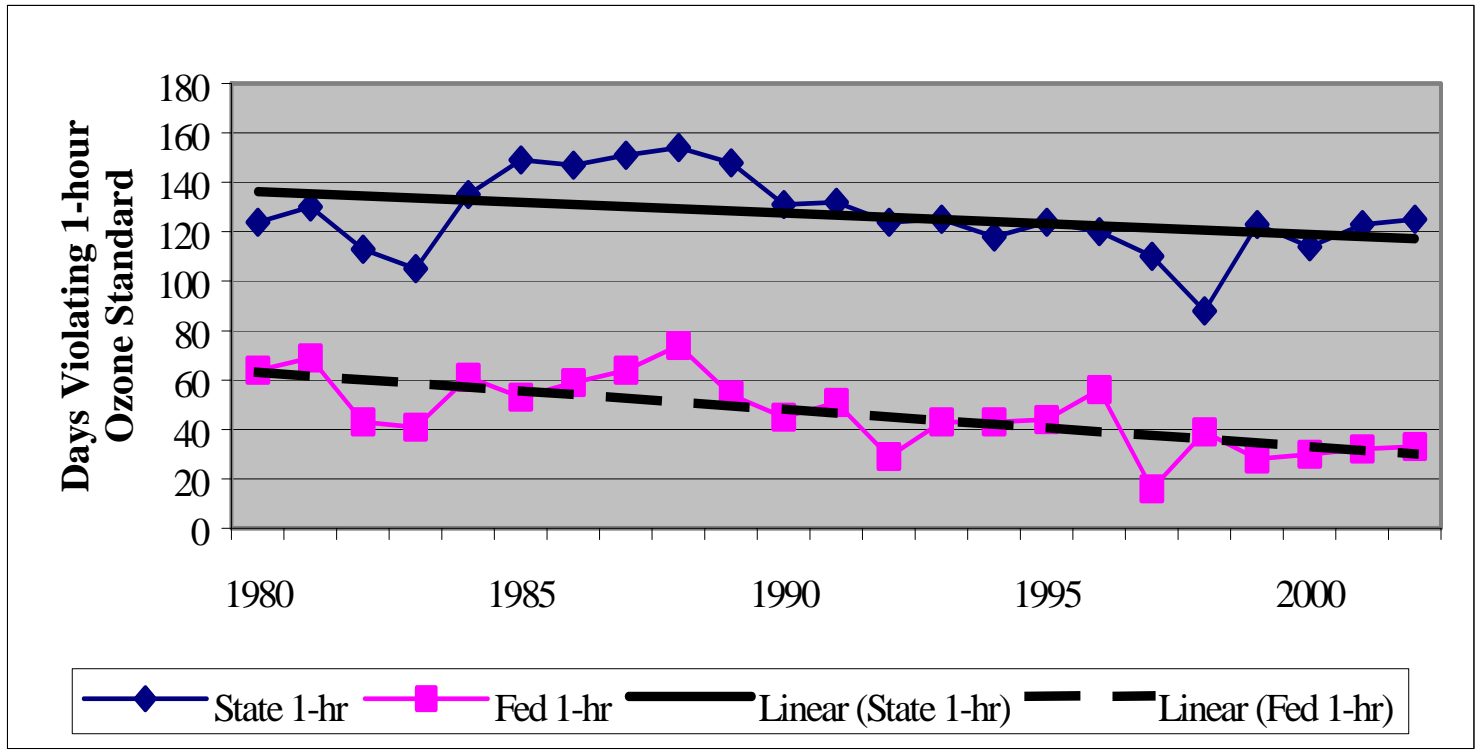




\section{Figure IV-4: Days Violating Federal 1-Hour Ozone Standard SJVUAPCD, 1990-2002}

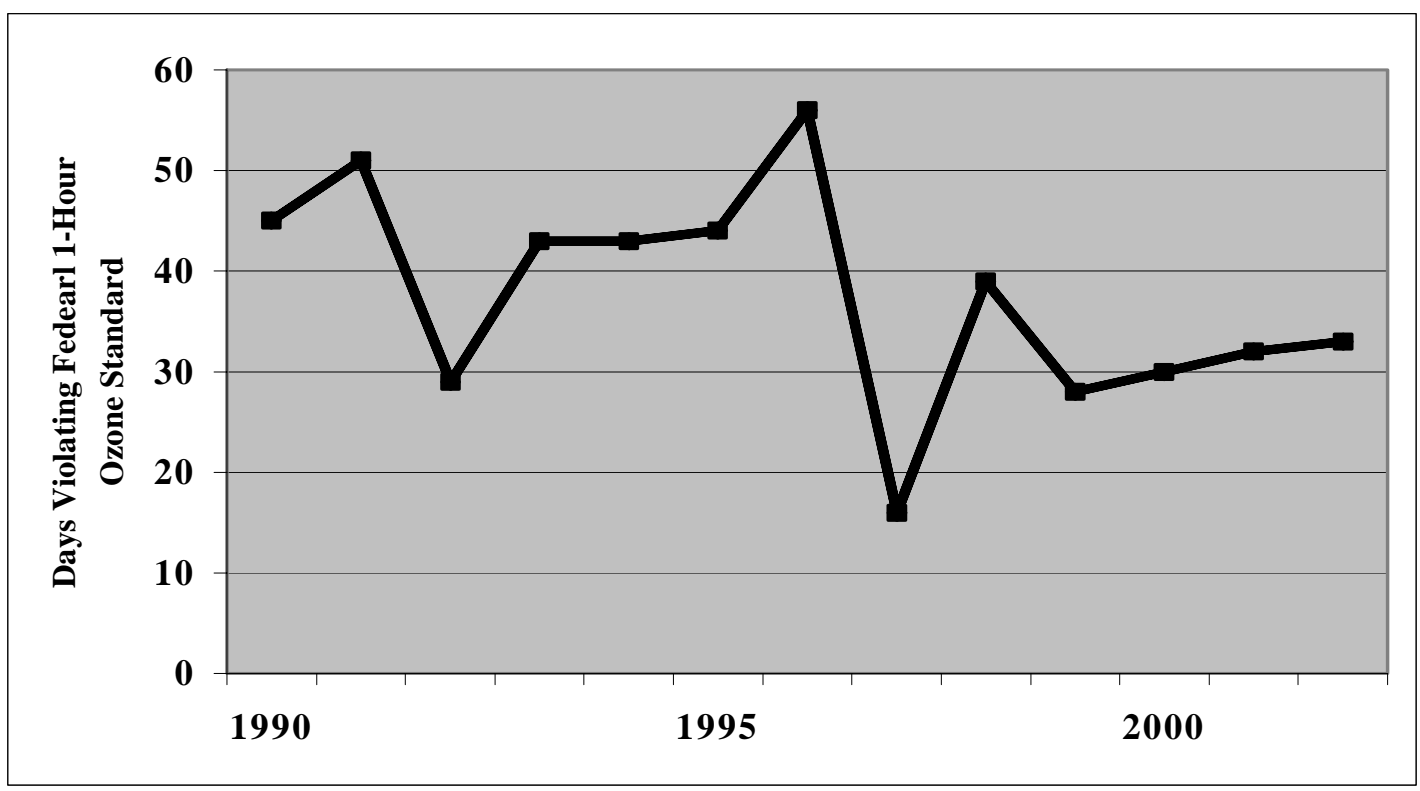

In June 1995, the Bay Area was designated as attained the 1-hour ozone NAAQS

after several clean years (see Figure IV-2). Alas, within one week of that designation, the Bay Area experienced the first of eleven violations, an exceptionally poor ozone air quality season. After some delay, the EPA redesignated the Bay Area as nonattainment for the ozone NAAQS (EPA 1999a).

The EPA offers their assessment of air quality trends in the Bay Area and SJV in its annual National Air Quality and Emissions Trends Report. Tables IV-A and IV-B show peak observations of $\mathrm{O}_{3}$ and $\mathrm{PM}_{10}$ over the three year period, 1997-1999, and EPA's conclusion about trends over the past decade. 
Table IV-A: Central California Counties and Their Air Quality Conditions in 1999 (EPA 2001)

Values in bold exceed the NAAQS.

\begin{tabular}{|c|c|c|c|c|c|}
\hline $\begin{array}{l}\text { Air } \\
\text { District }\end{array}$ & Country & $\begin{array}{c}\mathbf{O}_{3} \\
\text { 1-Hour }\end{array}$ & $\begin{array}{c}\mathrm{O}_{3} \\
\text { 8-Hour }\end{array}$ & $\mathbf{P M}_{10}$ Wtd. AM & $\begin{array}{c}\mathbf{P M}_{10} \\
2^{\text {nd }} \text { Max. }\end{array}$ \\
\hline \multicolumn{6}{|c|}{ BAAQMD } \\
\hline & Alameda & 0.14 & 0.09 & 26 & $\overline{94}$ \\
\hline & Contra Costa & 0.13 & 0.09 & 26 & 89 \\
\hline & Marin & 0.10 & 0.06 & 22 & 66 \\
\hline & Napa & 0.11 & 0.08 & 19 & 54 \\
\hline & San Francisco & 0.07 & 0.05 & 27 & 70 \\
\hline & San Mateo & 0.08 & 0.05 & 27 & 75 \\
\hline & Santa Clara & 0.12 & 0.08 & 29 & 94 \\
\hline & Solano & 0.12 & 0.09 & 20 & 64 \\
\hline & Sonoma & 0.10 & 0.08 & 19 & 65 \\
\hline \multicolumn{6}{|c|}{ SJVUAPCD } \\
\hline & Fresno & 0.15 & 0.11 & 47 & 130 \\
\hline & Kern & 0.14 & 0.11 & 61 & 142 \\
\hline & Kings & 0.13 & 0.10 & 54 & 146 \\
\hline & Madera & 0.10 & 0.09 & ND & ND \\
\hline & Merced & 0.13 & 0.11 & IN & IN \\
\hline & San Joaquin & 0.13 & 0.09 & 37 & 123 \\
\hline & Stanislaus & 0.11 & 0.09 & 43 & 137 \\
\hline & Tulare & 0.13 & 0.11 & 56 & 137 \\
\hline
\end{tabular}

$\mathrm{O}_{3}$ 1-Hour $=$ Highest $2^{\text {nd }}$ Daily Max. 1-Hour Concentration (parts permillion)

$\mathrm{O}_{3}$ 8-Hour $=$ Highest 4th Daily Max. Concentration (parts per million)

$\mathrm{PM}_{10}$ Wtd. $\mathrm{AM}=$ Highest weighted annual mean

$\mathrm{PM}_{10} 2^{\text {nd }}$ Max. $=$ Highest second maximum 24-hour concentrations.

IN = Incomplete data; ND = No data

Values in bold exceed the NAAQS, which are listed in Table V-A. California standards are in

Table V-B. 
Table IV-B: Central California Metropolitan Statistical Areas and Their Air Quality Trends, 1990-1999 (EPA 2001)

\begin{tabular}{|c|c|c|c|c|c|}
\hline $\begin{array}{l}\text { Air } \\
\text { District }\end{array}$ & $\begin{array}{l}\text { Metropolitan } \\
\text { Statistical Area }\end{array}$ & $\begin{array}{l}\text { 1-Hour } \\
\text { Ozone } \\
\text { Trend }\end{array}$ & $\begin{array}{l}\text { 8-Hour } \\
\text { Ozone } \\
\text { Trend }\end{array}$ & $\begin{array}{l}\mathbf{P M}_{10} 9^{\text {th }} \\
\text { Percentile }\end{array}$ & $\begin{array}{c}\text { PM }_{10} \\
\text { Annual } \\
\text { Mean }\end{array}$ \\
\hline \multicolumn{6}{|c|}{ BAAQMD } \\
\hline & Oakland & NS & NS & Down & Down \\
\hline & San Francisco & NS & NS & Down & Dowr \\
\hline & San Jose & NS & NS & Down & Dowr \\
\hline & Santa Rosa & Up & Up & Down & Dowr \\
\hline \multicolumn{6}{|c|}{ SJVUAPCD } \\
\hline & Bakersfield & NS & NS & Down & Dowr \\
\hline & Fresno & NS & NS & NS & Dowr \\
\hline & Salinas & NS & NS & Down & Dowr \\
\hline & \begin{tabular}{|l|} 
Stockton-Lodi \\
\end{tabular} & NS & NS & Down & Dowr \\
\hline
\end{tabular}

1-Hr Ozone = Highest $2^{\text {nd }}$ Daily Max. 1-Hour Concentration (parts per million)

8-Hr Ozone $=$ Highest 4th Daily Max. Concentration (parts per million)

NS = No statistically significant trend; Down = Downward trend; Up $=$ Upward trend

The EPA sees little trend, finding "no significant” trend, downward or up, for any of

the major metropolitan areas in the Bay Area or the Central Valley. The SJVUAPCD has a slightly different interpretation of progress, stating,

[T] here has been no improvement in decreasing annual maximum [for 1hour ozone]. However, there has been considerable improvement in other statistics, particularly in the number of days and the number of hours over the standard. (SJVUAPCD 2001b, page 2-10).

Some staff at the SJVUAPCD contend controls have relocated peak ozone away from population centers. In their 2001 Triennial Progress Report (01 TPR), the SJVUAPCD presents graphical trends for Expected Peak Day Concentration (EPDC) $)^{63}$ at 23 monitoring sites operating continuously since 1986 and concludes,

The EPCDs for 17 of the 23 sites in the SJV [Air Basin] decreased (some by only one part per billion), five out of 23 increased, and one site had data for only 1999 (SJVUAPCD 2001a, page 4).

\footnotetext{
63 The SJVUAPCD choice of metrics to assess air quality is based on guidance from the California Air Resources Board suggesting the use of expected peak day concentration, per-capita annual exposure, and per-unit-area annual exposure (CARB 1993).
} 
Other data presented in the progress report indicate no or negative progress toward cleaner air. Aggregate hours above the state standard, for example, are shown in Figure IV-5. Although presented, these data are not discussed in the report. The Draft 02 Ozone Plan compares two three-year periods, 1988-1990 and 1998-2000. Finding that the earlier year averaged 58 violations of the federal ozone standard per year compared with merely 32 per year in the latter period, the SJVUAPCD concluded, "This is a 45 percent improvement. This represents a significant reduction in population exposure to high levels of ozone” (SJVUAPCD 2001b). Thus, conclusions appear to be based upon what data are considered and how they are analyzed.

Figure IV-5: Hours Exceeding the State 1-Hour Standard in the SJVUAPCD, 1990-1999 (SJVUAPCD 2001a)

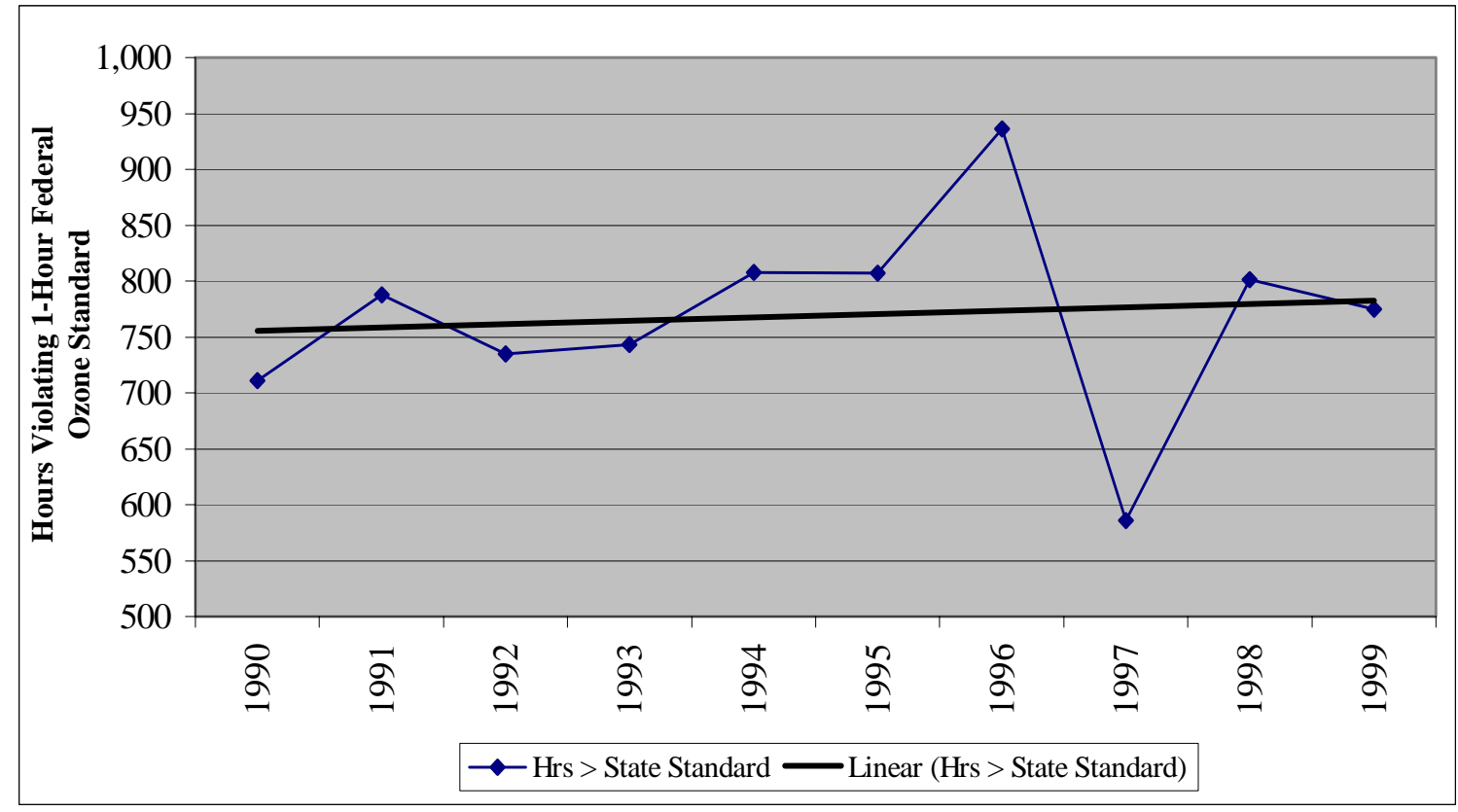

The 2001 TPR also presents trends for population weighted and area weighted exposure, which both show downward trends. The figures include confidence intervals. This is an example of specific mention of uncertainty. The treatment and explanation is 
informal and incomplete, using unfamiliar concepts of “documented progress” and "native variability".

The treatment of uncertainty is incomplete and therefore misleading. By referring to confidence intervals, a level of reliability is suggested. Strictly speaking, other uncertainties not considered would influence the confidence interval. Furthermore, the SJVUAPCD provides sparse description of the methods used to calculate confidence intervals, so it is not possible to know what they are confident about. The SJVUAPCD does, however, direct interested readers to CARB guidance defining "native variability" (see CARB 1993). For one not trained in statistics, or even a well-trained analyst without access to CARB's descriptions, it is not possible to know or to critique the conclusion in the TPR.

Unfortunately, exposure trends are not directly relevant because the current form of the standard focuses on ambient ozone concentration peaks averaged over one hour. Another shortcoming of the per capita exposure calculation is that it actually measures ambient concentrations outside of homes, not individuals’ exposure. Air quality indoors is very different from outdoor air quality and people spend an average of 90 percent of their time indoors (Jenkins et al. 1992), so the indicator is misleading. ${ }^{64}$

The issue of available data is a recurring theme amongst scientists studying air quality. Modelers and planners would like an extensive observational database with which to evaluate air quality conditions and trends, and to input into and evaluate models.

\footnotetext{
${ }^{64}$ Despite its limitations, industrial emissions sources support the use of per capita and per unit-area exposure as air quality indicators (for examples, see Ziman (1993c) and WSPA (1996)). These measures are believed to be more relevant because they are the product of people (or land use), concentration and time. However, little is known about personal exposure. Indoor sources, such as cigarettes and stoves, can
} 
Unfortunately, the time and resources necessary to create such a database are rarely available. If there is one exception, it is in the SJV, where air quality studies, including intensive field studies, have produced a state-of-the-science modeling system and the most robust observational database available. It is still not enough. In 2001, after over 10 years and \$20 million invested in SJV air quality studies, the Technical Advisory Committee (Technical Committee) wrote a memo recommending development and implementation of an aerometric monitoring network to:

- Provide the capability to model any day of the year

- Support data and trends analyses

- Develop an integrated air quality management strategy that addresses precursors to ozone and particulate matter, as well as toxic air pollutants.

The Technical Committee wrote,

While modeling has been the primary tool for development of SIPs, the trend is to view modeling as one of a number of tools to use in design of an effective strategy. Moreover, there is a need to have actual data to see if the strategy developed through modeling is actually leading to improvements in air quality (TAC 2001).

\section{Emissions}

During the application of the SARMAP modeling system in 1994, there were nine documented updates to the 1990 baseline emissions estimate (SJVUAPCD 1994). The estimate included both Bay Area and SJV emissions. As shown in Figure IV-6, the estimate of total reactive organic gas (ROG) emissions declined by approximately 1000 tpd as a result of the updates, whereas the $\mathrm{NO}_{\mathrm{x}}$ estimate changed very little. However, 
source contributions did change with the updates. The SJVUAPCD and CARB were forthcoming about changes and uncertainties during the updates in 1994, including:

- Isoprene emissions were scaled downward by a factor of five

- Corrected errors associated with locating emissions spatially

- Corrected motor vehicle emissions estimate to account for new emissions factors values and driving activity

Agricultural internal combustion engines were not included.

Figure IV-6: Generations of the SARMP 1990 Baseline Emissions Inventory

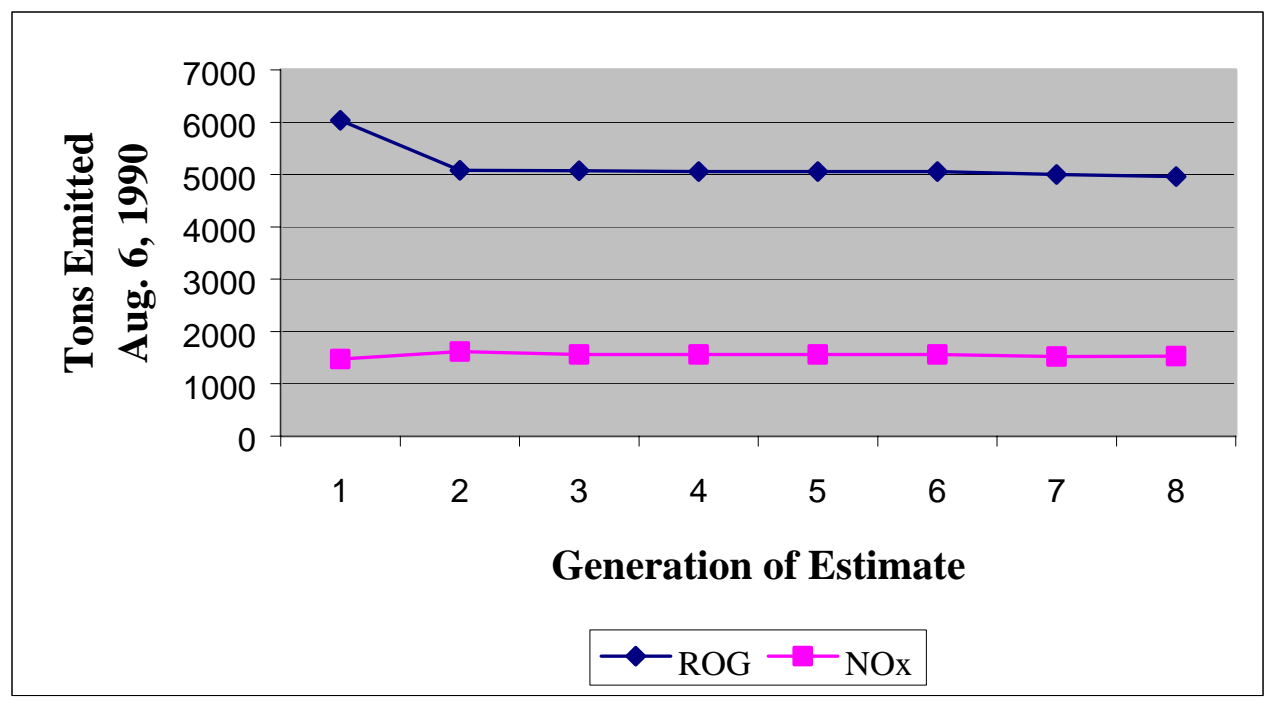

The most recent updates to the emissions estimate are for planning to produce the 2002 amendment to the 94 Ozone Plan. The revised inventory is summarized in Figures IV-7 and IV-8, but it includes only anthropogenic sources.

Large stationary (or “point”) sources are large smoke stacks, such as industrial facilities. Area (or “nonpoint”) sources are numerous small sources (e.g., restaurants, dry cleaners, residential wood stoves) or large surfaces (e.g., agricultural fields, dusty 
roadways). Mobile sources include automobiles, trucks, trains, boats, planes and off-road vehicles, such as construction and farm equipment.

Figure IV-7: 1990 ROG Emissions Inventory for Draft 2002 Ozone Plan (SJVUAPCD 2001b; Merchen 2002)

(Tons Per Day)

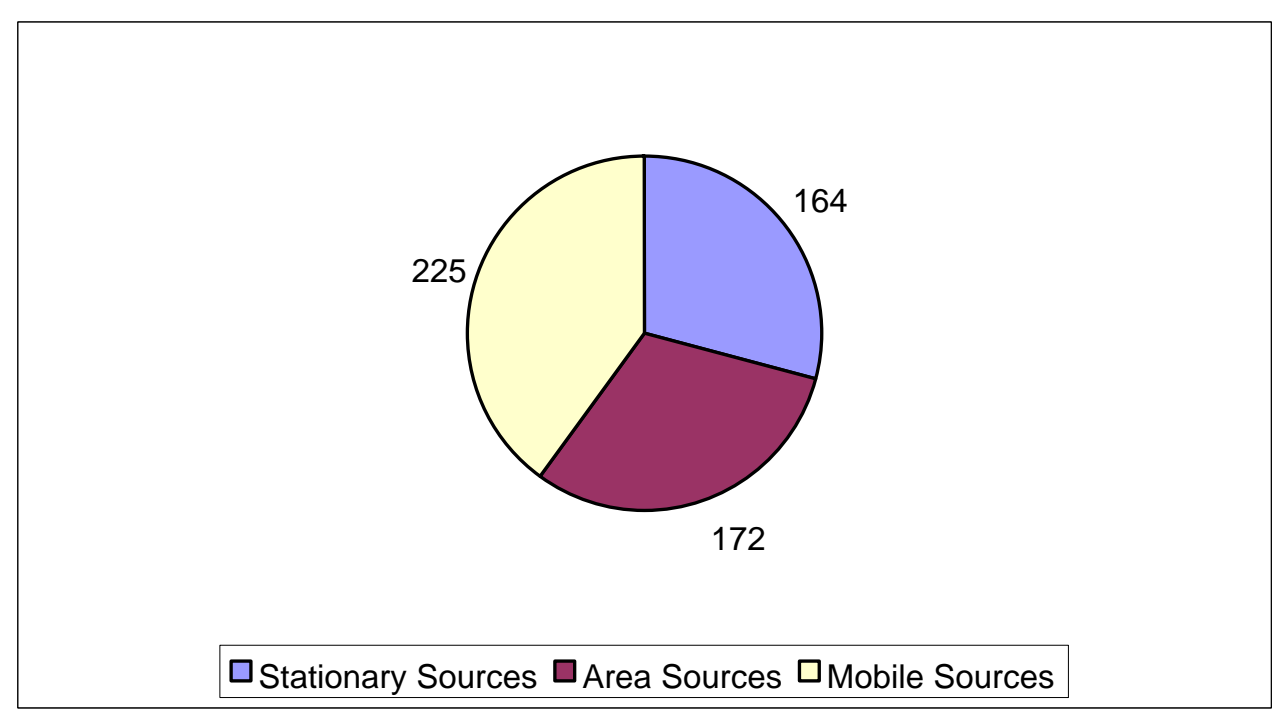

Figure IV-8: $1990 \mathrm{NO}_{\mathrm{x}}$ Emissions Inventory Used for Draft 2002 Ozone Plan (SJVUAPCD 2001b; Merchen 2002)

(Tons Per Day)

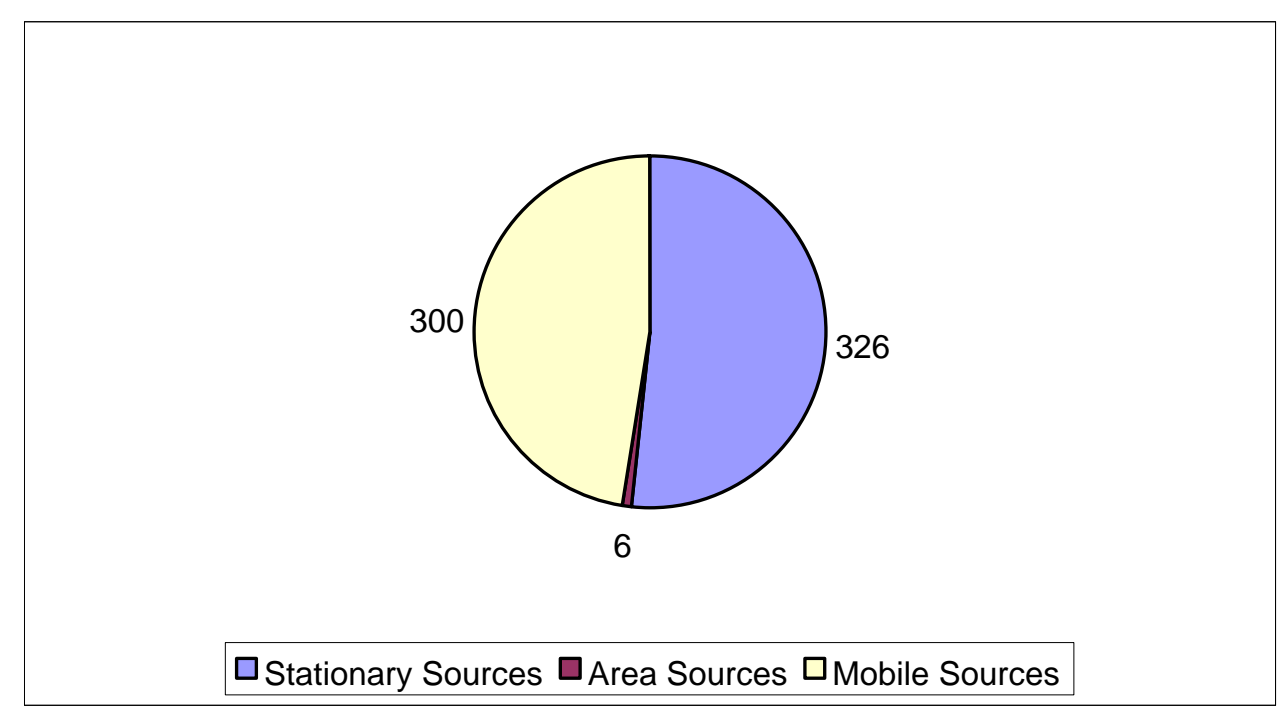


Figures IV-9, IV-10 and IV-11 summarize CARB's preliminary estimates of the changes to the ROG inventory for the SJV area. ${ }^{65}$ Major differences between the 1990 baseline inventory used in 1994 and the current best estimate of 1990 emissions include:

- 34 additional tons per day (tpd) of ROG emissions from stationary sources, mostly oil and gas production.

- 118 additional tpd of ROG emissions from mobile sources, including nearly 90 tpd from on-road vehicles, over 10 tpd from aircraft, and less than five additional tpd from off-road vehicles, farm equipment and fuel storage. The ROG emissions estimate from trains declined by a few tpd. The major difference in the on-road vehicle emissions estimate is due to the use of a new motor vehicle emissions model, EMFAC7.

- 68 additional tpd of ROG from area sources, including 75 tpd from livestock waste, pesticides and fertilizers, offset by a downward adjustment of emissions from architectural coatings and solvents, asphalt paving and roofing, and utility equipment.

In addition, biogenic ROG emissions were approximately halved throughout the SARMAP domain in the most recent update. Isoprene emissions were reduced by a factor of two, terpene emissions by a factor of four, and the other biogenic ROG emissions by a third (CARB 2001a).

\footnotetext{
65 These estimates are preliminary because the CARB and SJVUAPCD have not completed their updates to the inventory. The most significant anticipated changes will be associated with the motor vehicle emissions inventory once a newer version of the motor vehicle emissions model, EMFAC, is exercised. The SJVUAPCD’s draft 2002 amendment to their Ozone Attainment Plan includes a motor vehicle emissions inventory that will be outdated by the time the plan is made final.
} 
Figure IV-9: 1990 Baseline Emissions Estimate for SJV Air Basin: Stationary Source ROG Difference between 1994 and Draft 2002 Ozone Plans

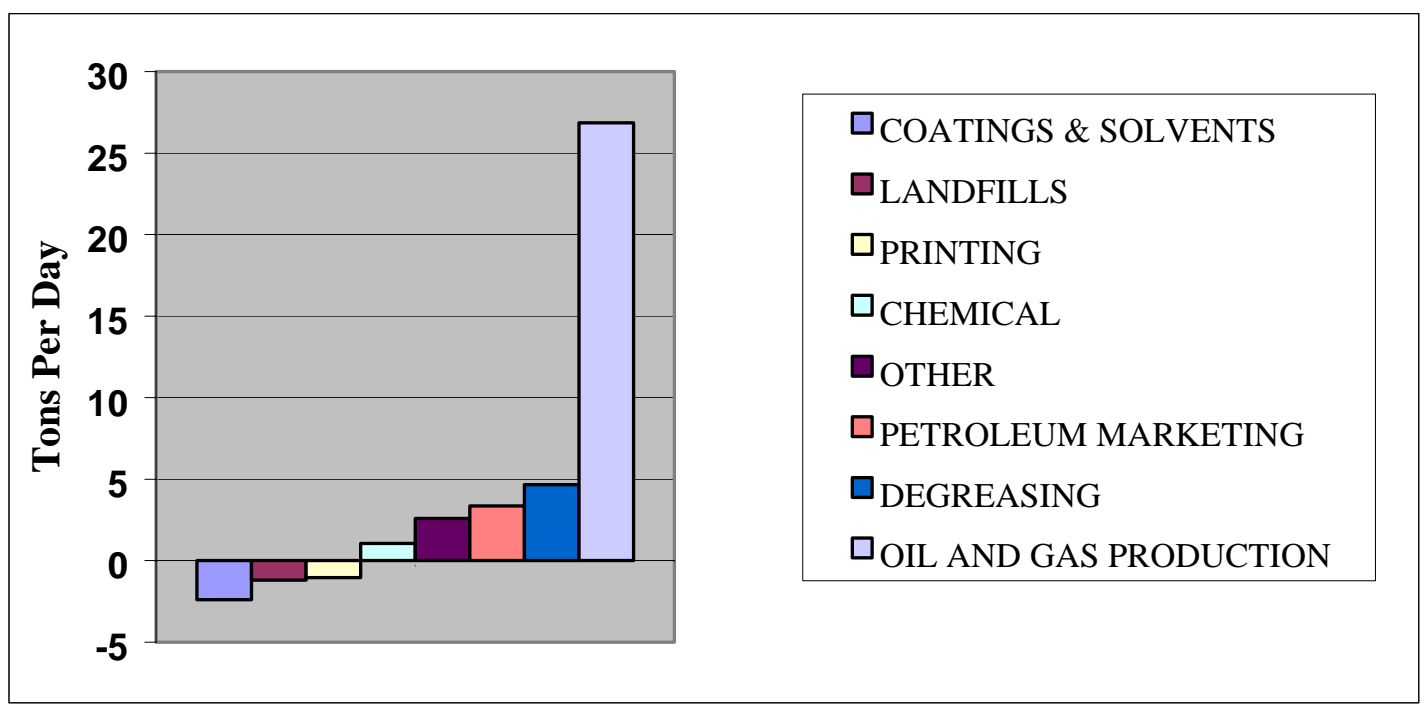

Figure IV-10: 1990 Baseline Emissions Estimate for SJV Air Basin: Mobile Source ROG Difference between 1994 and Draft 2002 Ozone Plans

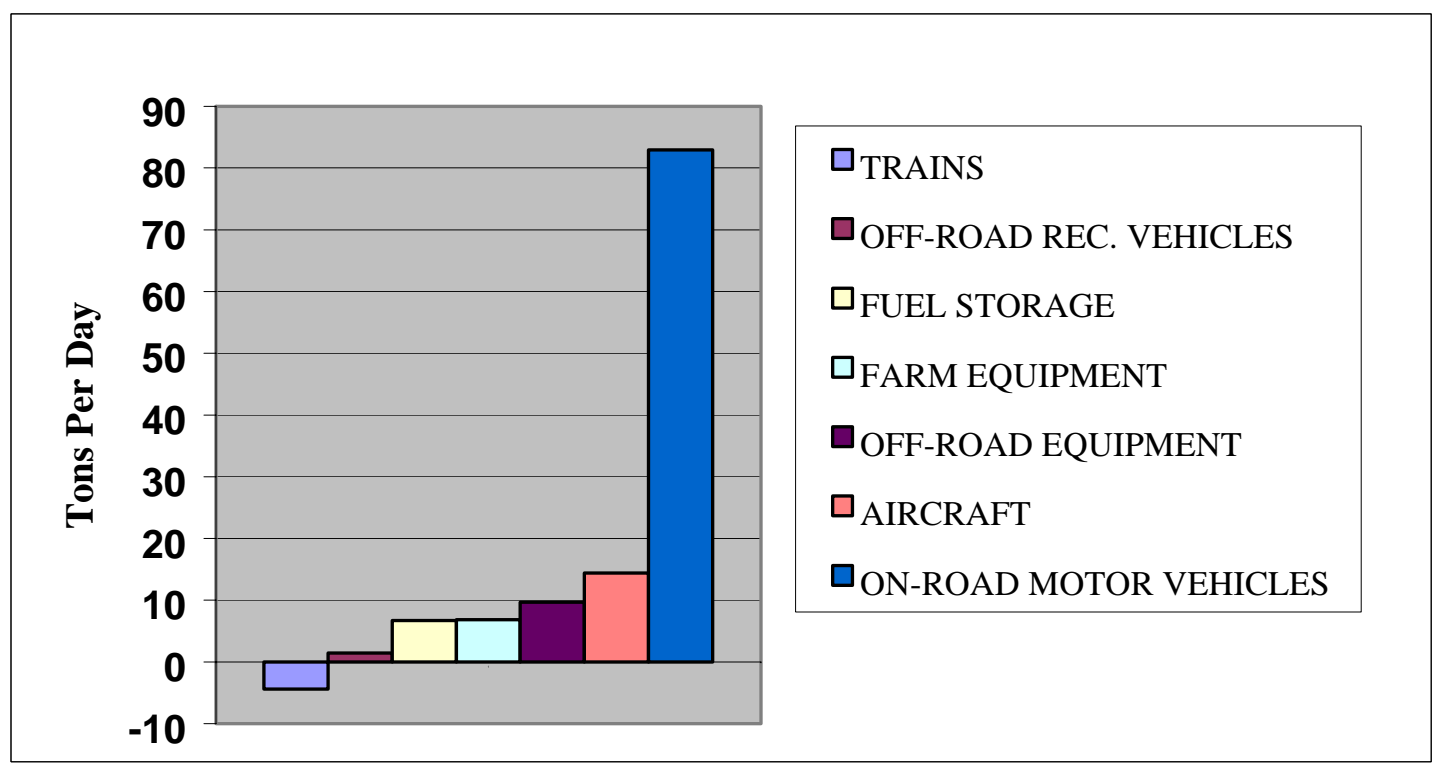


Figure IV-11: 1990 Baseline Emissions Estimate for SJV Air Basin: Area Source ROG Difference between 1994 and Draft 2002 Ozone Plans

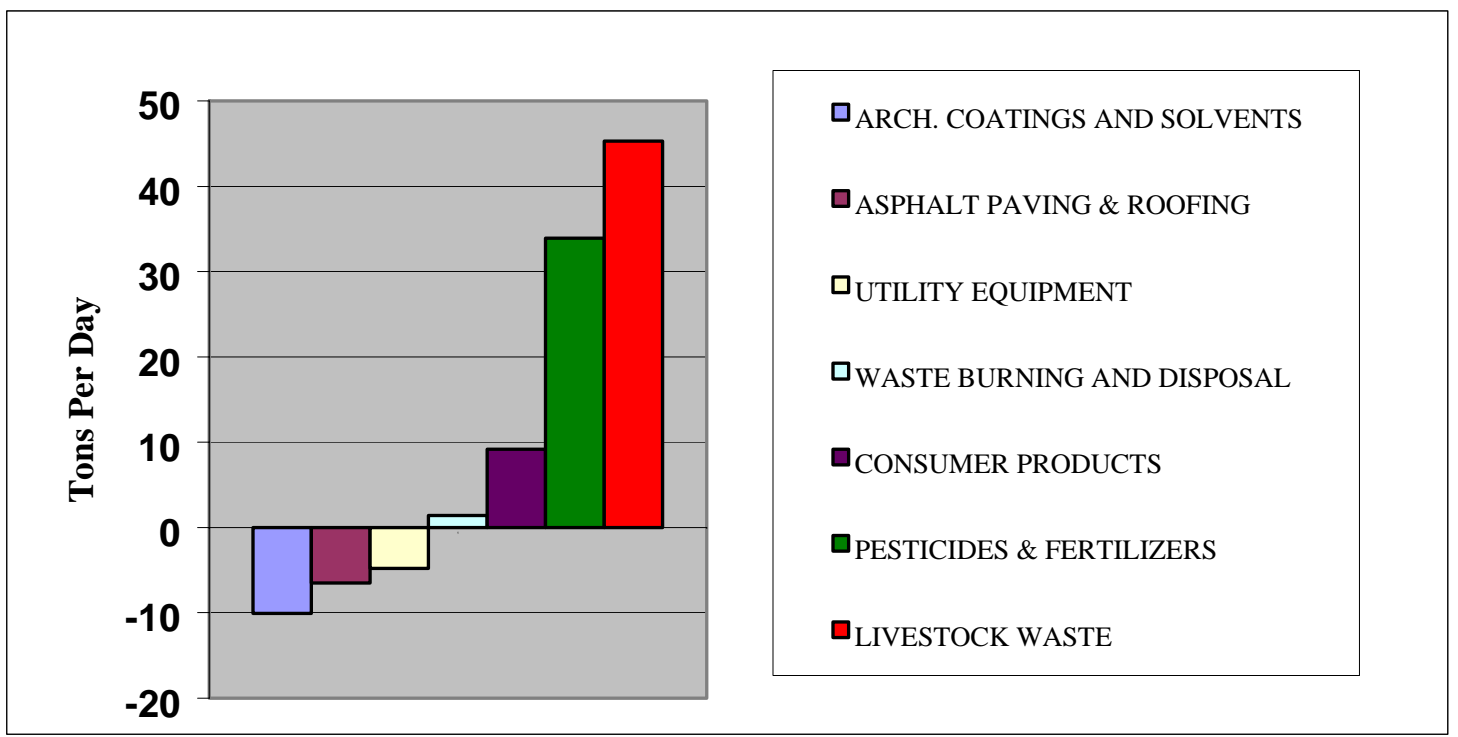

Overall, the new estimate of emissions of both $\mathrm{NO}_{\mathrm{x}}$ and ROG in 1990 are much higher, as shown in Figures IV-12 and IV-13.

Figure IV-12: 1990 and $1999 \mathrm{NO}_{\mathrm{x}}$ Emissions Estimates in SARMAP Domain: Difference between 1994 and Draft 2002 Ozone Plans

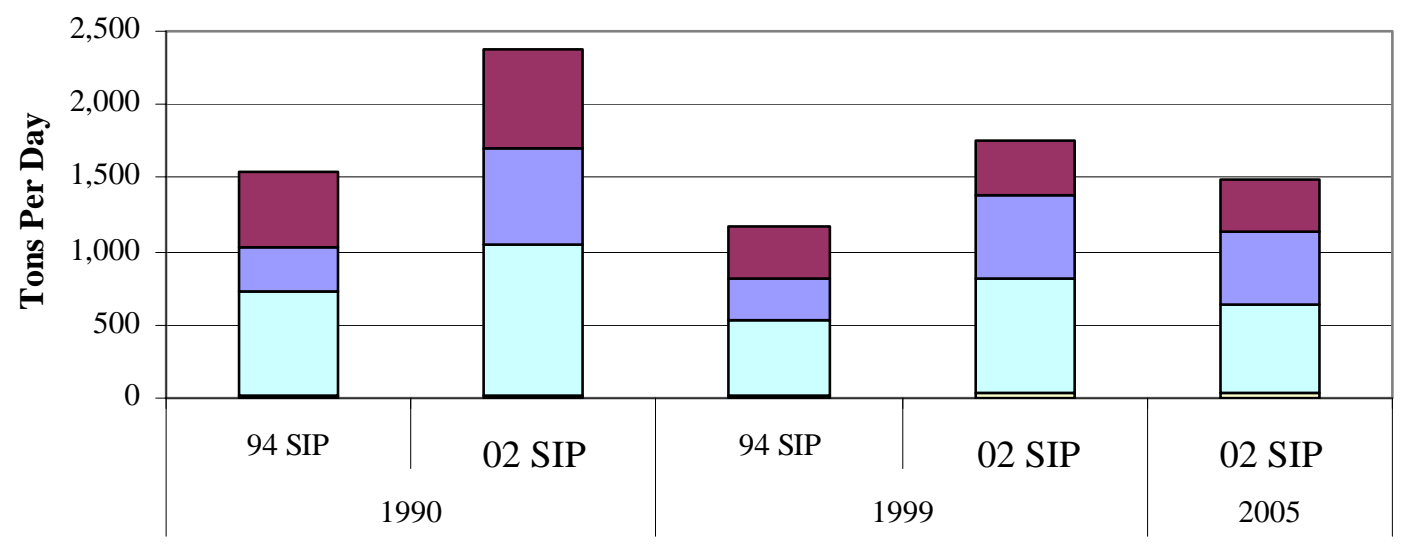




\section{Figure IV-13: 1990 and 1999 ROG Emissions Estimates in SARMAP Domain:}

Difference between 1994 and Draft 2002 Ozone Plans

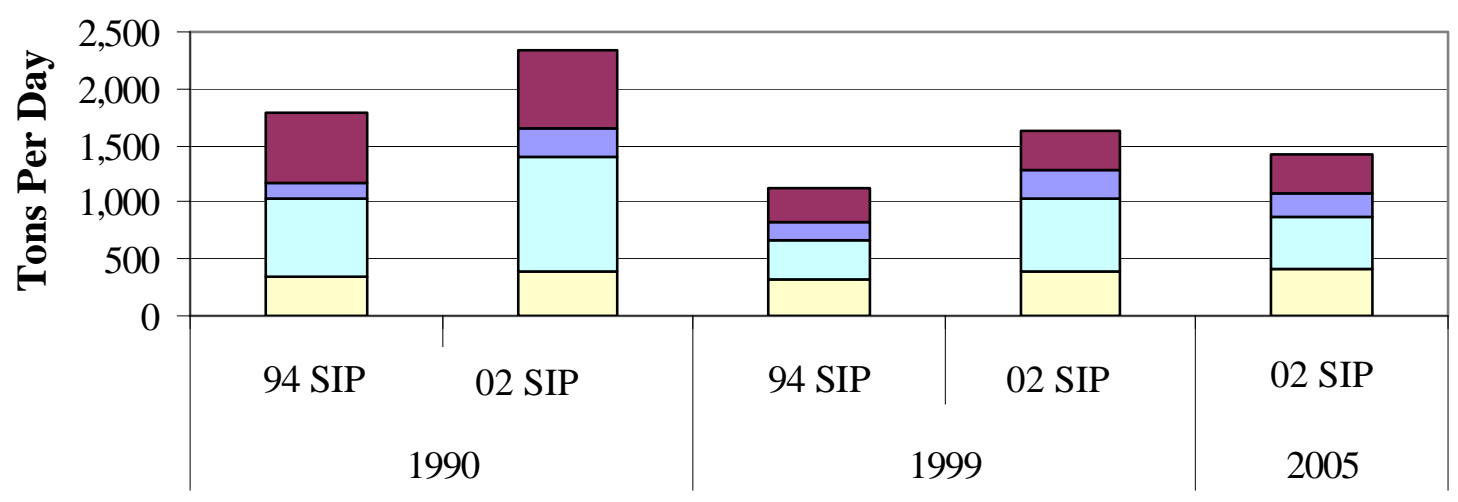

The implications of the updated emissions estimates are a more significant portion of emissions come from mobile and area sources, such as livestock waste and farming equipment. The updates further implicate emissions from agricultural activities including:

- farm equipment accounts for 43 percent (99 tpd) of the additional NOx emissions from mobile sources

- "food and agriculture" emissions total 10 percent (12 tpd) of the additional NOx from stationary sources

- livestock waste accounts for 12 percent of the estimated ROG emissions

The emissions estimate update indicates that the estimates are uncertain. The latest estimate for 1999 emissions is approximately one-third higher than the projections used in the 94 Ozone Plan. The large change suggests that the inventory is now more accurate. However, the experience with adjusting emissions in 1994, see Figure IV-6 and 
subsequent discussion, confirms that "best estimates" may nonetheless be uncertain. This is true for the estimate being used for the 2002 ozone planning cycle, even though they are more inclusive than the 1994 estimates (Marr 2002b).

\section{Emissions Controls}

Although the first round of stationary source controls were set forth in the SJV's 1991 Air Quality Attainment Plan , state-wide motor vehicles emissions standards had long been required by CARB. The 94 Ozone Plan lists control measures from the 1991 plan that would reduce VOC and $\mathrm{NO}_{\mathrm{x}}$ emissions by 75 and 142 tons per day, respectively, by 1999. The most substantive VOCs and $\mathrm{NO}_{\mathrm{x}}$ rules are listed in Table IV-C. The biggest reductions come from stationary industrial sources, notably those of the petroleum industry. However, these are reductions from sources within the SJVUAPCD’s jurisdiction. Emissions from motor vehicles, pesticide use and consumer products are within state or federal jurisdiction, so they are not listed in Table IV-C.

Table IV-C: Control Rules from 1991 AQMP for the SJV (SJVUAPCD 1994)

\begin{tabular}{|l|l|c|c|c|}
\hline Number & Title & $\begin{array}{c}\text { Full } \\
\text { Implementation } \\
\text { Date }\end{array}$ & $\begin{array}{c}\text { VOC } \\
\text { Reductions } \\
\text { (tpd) }\end{array}$ & $\begin{array}{c}\text { NO } \\
\text { Reductions } \\
\text { (tpd) }\end{array}$ \\
\hline 4401 & $\begin{array}{l}\text { Steam-enhanced Crude Oil } \\
\text { Production Well Vents }\end{array}$ & $5 / 1 / 95$ & 19.32 & N/A \\
\hline 4402 & Crude Oil Production Sumps & $5 / 1 / 97$ & 20.33 & N/A \\
\hline 4403 & $\begin{array}{l}\text { Components serving Oil \& Gas } \\
\text { Production Facilities }\end{array}$ & $11 / 1 / 91$ & 4.55 & N/A \\
\hline 4601 & Architectural Coatings & $9 / 1 / 94$ & 3.41 & N/A \\
\hline 4602 & Vehicle Refinishing Operations & $1 / 1 / 95$ & 5.58 & N/A \\
\hline 4623 & Storage of Organic Liquids & $1 / 1 / 96$ & 13.2 & N/A \\
\hline 4701 & $\begin{array}{l}\text { Stationary IC Engines - Central } \\
\text { and Western Kern Counties }\end{array}$ & $12 / 31 / 95$ & N/A & 35.90 \\
\hline 4305 & $\begin{array}{l}\text { Boilers, Process Heaters and } \\
\text { Steam Generators }\end{array}$ & $12 / 31 / 00$ & N/A & . \\
\hline
\end{tabular}


Two factors create increasing impetus to control emissions from area and mobile sources. First, stationary source controls are insufficient to meet air quality goals. A finding of the SJVAQS was that reductions on the order of 50 to 75 percent will necessary to meet the state ozone standard. Second, the 2002 update to the emissions inventory resulted in higher estimates of emissions from mobile and area sources. The higher emissions estimate, combined with decreasing emissions from stationary sources, makes mobile and area sources an increasingly significant portion of total emissions. Yet another reason to extent controls on non-point sources is the need to meet ambient standards for particulate matter. This latter issue is discussed at length in the next section.

One point of contention is whether or not different sectors are being unfairly burdened with emissions controls. A comparison of emissions in 1990 and 2001 does indicate that the largest reductions have been achieved in the petroleum and mobile source sections, as shown in Table IV-D.

The table also shows that emissions due to oil and gas production are now lower than those associated with food and agricultural processing. There is no explicit statement in any of the legislation or guidance stating that control burdens must be shared equitably. Agricultural activities are excused explicitly from air quality controls (EPA 2003). 


\section{Table IV-D: Selected Emissions Reductions from 1990 to 2001 in San Joaquin Valley APCD (CARB 2002)}

\begin{tabular}{|l|c|c|c|c|}
\hline Source Category & $\begin{array}{l}\text { 1990 Emissions } \\
\text { (tons per day) }\end{array}$ & $\begin{array}{l}\text { 2001 Emissions } \\
\text { (tons per day) }\end{array}$ & $\begin{array}{l}\text { Net } \\
\text { Reduction }\end{array}$ & $\begin{array}{l}\% \text { Total } \\
\text { Change }^{66}\end{array}$ \\
\hline Total for SJVUAPCD & 796 & 545 & $251^{67}$ & N/A \\
\hline Oil and Gas Production & 126 & 22 & 251 & $100 \%$ \\
\hline $\begin{array}{l}\text { Manufacturing and } \\
\text { Industrial }\end{array}$ & 39 & 29 & 104 & $41 \%$ \\
\hline $\begin{array}{l}\text { Food and Agricultural } \\
\text { Processing }\end{array}$ & 39 & 35 & 10 & $4 \%$ \\
\hline $\begin{array}{l}\text { Service and } \\
\text { Commercial }\end{array}$ & 39 & 31 & 4 & $2 \%$ \\
\hline $\begin{array}{l}\text { On-Road Motor } \\
\text { Vehicles }\end{array}$ & 323 & 140 & 84 & $33 \%$ \\
\hline $\begin{array}{l}\text { Off-Road Mobile } \\
\text { Sources }\end{array}$ & 184 & & 8 & $3 \%$ \\
\hline
\end{tabular}

Rather than equity, "feasibility” is the primary criteria for determining emissions control burdens. Feasibility is defined in terms of technical and economic viability, but is left to the air districts to determine (Section 41650 (b) of CCAA 2001). Thus, one sector of emissions sources may be required to reduce emissions more than another simply because available controls are deemed feasible.

\section{Particulate Matter Conditions in the SJV and Bay Area}

Particulate matter (PM) is comprised of liquid or solid phase aerosols that can remain suspended in the air for a period of hours to years. It can cause adverse health effects when inhaled, visibility impairment by absorbing and scattering light, and materials and ecological damage through acidic deposition. Trapping of infrared radiation by ambient PM can also influence global and regional climate (Seinfeld and Pandis 1998; Menon, Hansen et al. 2002).

\footnotetext{
${ }^{66}$ Percentages sum to larger than $100 \%$ because total net reductions are less than total reductions because emissions from some categories increase during the period 1990-2001.

${ }^{67}$ Total reductions is less than sum of reductions from individual categories because some categories
} 
As described in the Regulatory Setting chapter, the NAAQS for particulate matter 10 microns in diameter and smaller $\left(\mathrm{PM}_{10}\right)$ was augmented by EPA in 1997 to include a standard for $\mathrm{PM}_{2.5}$ (“fine PM"). The additional standard was EPA's response to epidemiological studies associating health impacts with the smaller PM.

Particulate air quality science is more complex than ozone. Not all PM is alike; it varies by size, shape, chemical composition, deposition velocity, and, presumably, health effects. It may be comprised of many different pollutant species, emitted directly (e.g., formed during combustion) or formed secondarily in the atmosphere. Secondary PM formation results from nucleation and from oxidation chemistry occurring in both gas and liquid phases, and involves ubiquitous gaseous precursors, including VOCs and $\mathrm{NO}_{\mathrm{x}}$ (Seinfeld and Pandis 1998). Ambient concentrations are affected by temperature and humidity, and may exist in liquid or solid form. It may deposit on surfaces and, consequently, may exist in much lower quantities indoors than outside (for example, see Lunden et al. 2003), or may be much higher due to indoor sources, such as heating, smoking or cooking (for example, see CARB 2001b). Furthermore, urban and rural PM can have different composition (Lehrman et al. 1998).

Yet another characteristic of PM in the SJV is that high PM concentrations occur in variety of meteorological conditions, violating standards in the summer, fall and winter. Comparatively, ozone is only a problem on hot, dry days occurring in the summer and early fall. 
Tables IV-A and IV-B show that $\mathrm{PM}_{10}$ standards are violated in both the SJV and the Bay Area, that $\mathrm{PM}_{10}$ concentrations are much higher in the SJV than the Bay Area, and that trends are downward in both air districts.

The $\mathrm{PM}_{10}$ problem may not seem to be as bad as the ozone problem, but this may not be the case for number of reasons. Poor PM air quality occurs throughout the SJV. From 1990 to 1995, approximately 45 percent of the monitors in the SJV exceeded the annual $\mathrm{PM}_{10}$ NAAQS. Over the same period, five percent of monitoring observations exceeded the 24-hour $\mathrm{PM}_{10}$ NAAQS (SJVUAPCD 1997). The new NAAQS for $\mathrm{PM}_{2.5}$ is also violated in the SJV. Both the average annual (chronic) and 24-hour (acute) PM standards are regularly exceeded for both $\mathrm{PM}_{10}$ and $\mathrm{PM}_{2.5}$ at numerous locations throughout the SJV (CARB 1997). These violations suggest that the PM air quality problem is both pervasive and severe.

In addition to complex PM science, PM source fractions vary significantly with the time of year. Geologic dust dominates observed ambient $\mathrm{PM}_{10}$ mass during the summer months. During the wintertime, secondary PM is significant.

\section{Emissions Controls}

Attaining standards may, like ozone, require reducing emissions from non-point sources. Much of the primary PM and secondary PM precursor emissions are generated by dispersed area, mobile and natural sources. As shown in Figure IV-14, area sources comprise an SJV-wide average of approximately 90 percent of $\mathrm{PM}_{10}$ emissions, which are predominately geologic dust. The importance of secondary PM in the SJV provides further impetus to reduce emissions of $\mathrm{NO}_{\mathrm{x}}$, since it leads to the formation of ammonium 
nitrate. Figures IV-8 and IV-15 show that area and mobile sources are also responsible for most emissions of ammonia $\left(\mathrm{NH}_{3}\right)$, and that mobile and stationary sources contribute most $\mathrm{NO}_{\mathrm{x}}$ emissions.

However, the significance of both primary and precursor emissions varies dramatically in space and time. An example of this variation is shown in Figure IV-16, which was generated using Chemical Mass Balance modeling of ambient PM samples collected in Corcoran and Bakersfield, two urban areas in the SJV. Although both urban sites exceed the $\mathrm{PM}_{10}$ NAAQS, the relative significance of sources is location specific. Similarly, the relative significance of emissions sources at a specific site varies at daily and seasonal time scales.

Figure IV-14: Sources of $\mathrm{PM}_{10}$ in the SJV (CRPAQS )

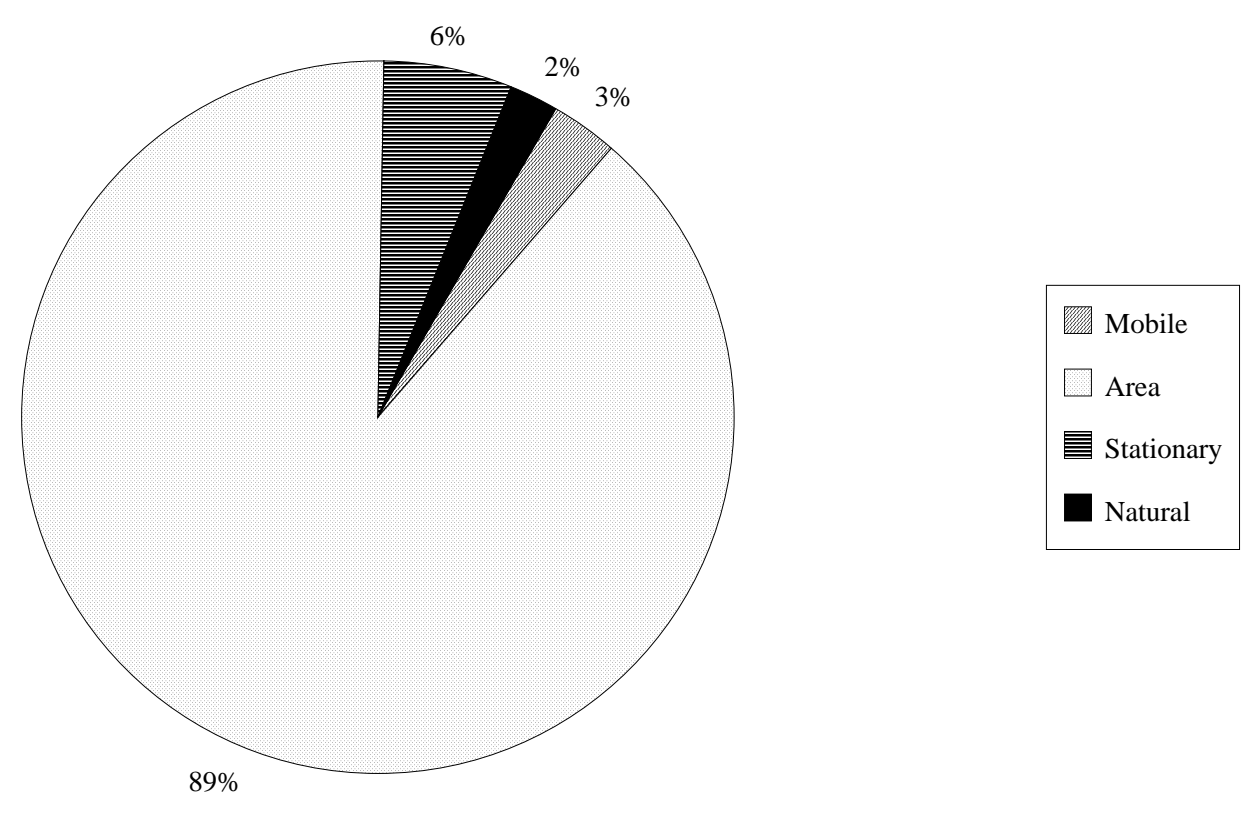


Figure IV-15: Sources of $\mathrm{NH}_{3}$ in the SJV (Coe 1998)
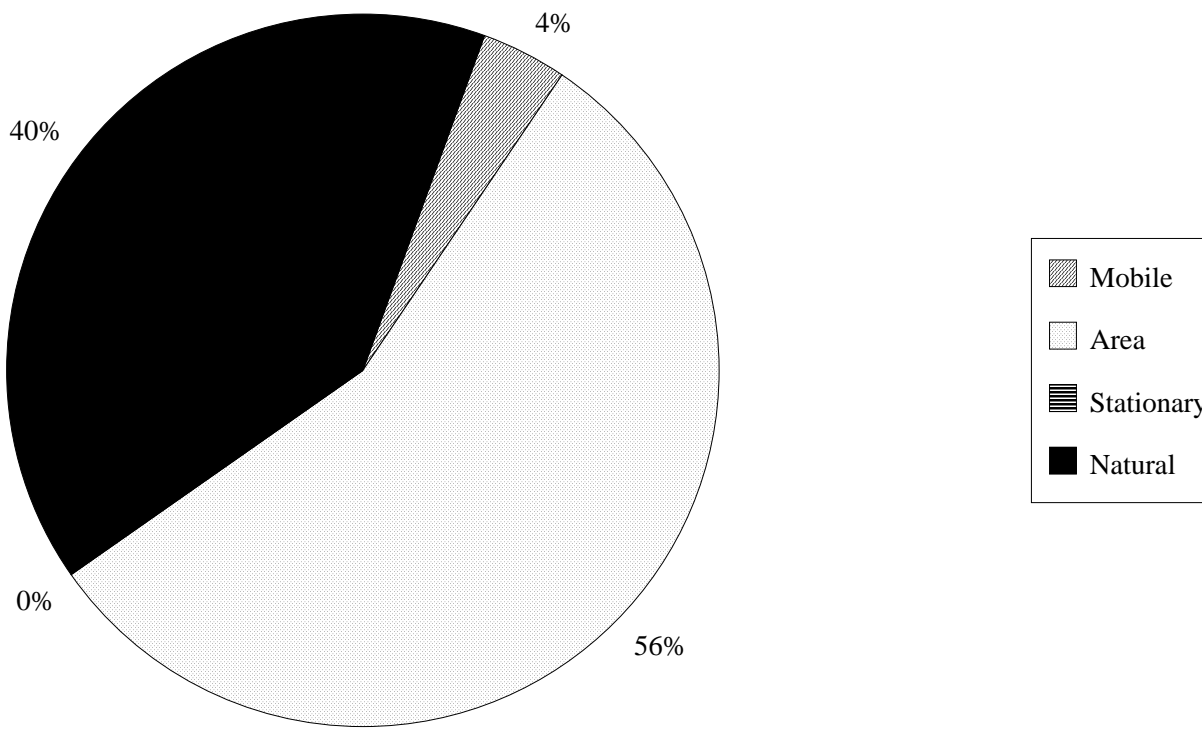

Figure IV-16: Variable Significance of PM Sources in Space and Time (Magliano 1998)

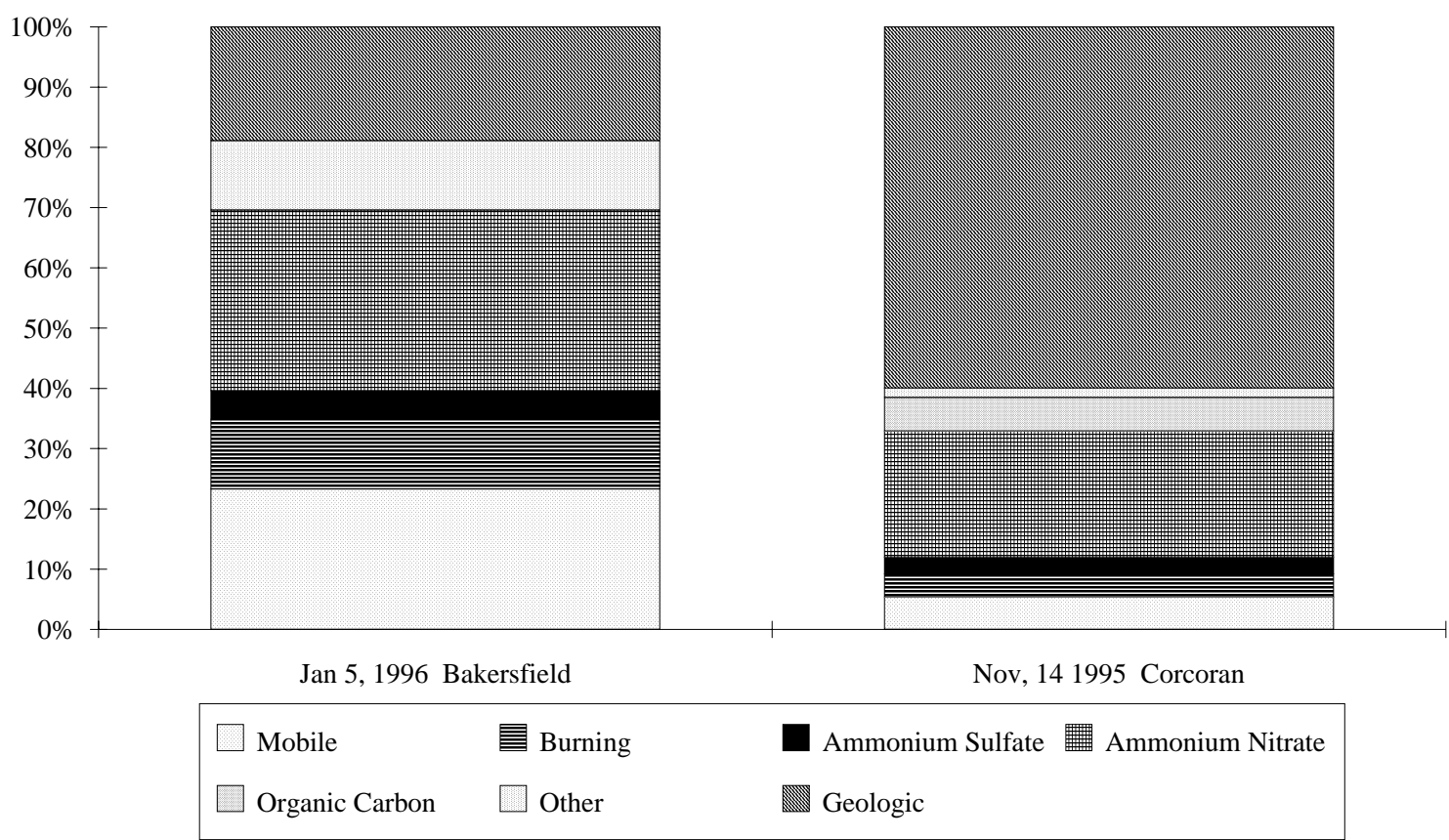


The significance of non-point sources represents a difficult challenge for planners. Point sources have been the traditional focus of control programs implemented by local air districts, whereas CARB has focused on reducing mobile source emissions. The provisions of the Federal Clean Air Act pertaining to particulate matter emissions specifically address point sources. Point sources with the potential to emit greater than 70 tons of $\mathrm{PM}_{10}$ annually are required to implement Reasonable Available Control Methods (RACM). In severe nonattainment regions, such as the SJV, these point sources must install Best Available Control Methods (BACM).

The FCAA also calls for establishment of RACM for "urban fugitive dust, and emissions from residential wood combustion and prescribed silvicultural and agricultural burning" and to "examine other categories of sources contributing to nonattainment of the PM-10 standard to determine whether additional guidance on reasonably available control measures and best available control measures is needed” (CAAA 1990).

BACM are insufficient to achieve attainment of the $\mathrm{PM}_{10}$ NAAQS in the SJV. Most BACM are designed to reduce emissions during high wind events, but the SJV experiences unacceptable ambient PM concentrations during calm days (SJVUAPCD 1997, page 7-1). In addition, control options are constrained by two sets of criteria: (1) "federal implementation criteria” and (2) de minimus, technological feasibility, and implementation cost criteria. For example, although the EPA identified 99 RACM for $\mathrm{PM}_{10}$ in Arizona as part of the $1998 \mathrm{PM}_{10}$ NAAQS attainment plan, only 10 measures met these criteria (Doris and Wicher 1998). 
The historical emphasis on controlling point sources is due to their recognizable contribution to air quality problems as well as the relative ease of implementing and monitoring point source controls. For example, whereas it is technically feasible to install equipment on a few smoke stack, it is more difficult to install controls on many residential wood stoves.

There is also a large contribution of natural and, hence, uncontrollable sources in the SJV. Natural sources are estimated to comprise 40 percent of all ammonia emissions (see Figure IV-15). The SJVUAPCD estimates that biogenic sources, such as vegetation, contribute significant hydrocarbon emissions in the region. Although no estimate of $\mathrm{NO}_{\mathrm{x}}$ emissions from soils is mentioned, they may be significant. Research and modeling indicate $\mathrm{NO}_{\mathrm{x}}$ emission from soils, though uncertain and seasonally-dependent, can comprise 50 percent of total emissions in some counties in the Midwestern U.S. and that agricultural uses of fertilizers are a key cause (Hall et al. 1996).

If the PM standards are going to be met in the SJV, controls of area and mobile sources will be necessary. These controls will impact individuals' activities, such as driving, and economically important industries, such as agriculture and trucking. It remains to be seen if the political will exists within the region to implement controls on the general population and industries with powerful political lobbies. The public's unwillingness to accept further controls on mobile emissions was painfully obvious in 1995 when obstinate drivers staged a rally in Sacramento and stopped traffic on San Francisco’s Bay Bridge to protest implementation of a more stringent vehicle inspection and maintenance program (Pattison 1996). 
Given population growth projections and the relationship between people and nonpoint sources of pollution, PM air quality may worsen if implemented controls are insufficient . Activities such as driving, construction and residential wood burning contribute to PM emissions in the region and will increase with population. Emissions reductions necessary to achieve PM standards are therefore likely to be significant, farreaching and costly.

Furthermore, through $\mathrm{NO}_{\mathrm{x}}$, there is a clear connection between ozone and PM. The other ozone precursor, VOCs, also contributes to atmospheric chemistry that leads to the formation of secondary PM, such as ammonium nitrate. Consequently, both ozone and PM concerns provide the impetus to control these pollutants. However, the relationships are not so simple, nor is the need to exact these controls in the SJV, or the Bay Area, clear. Past and ongoing research is intended to address these and other planning questions.

\section{Central California Air Quality Studies}

\section{SJVAQS and AUSPEX}

Numerous air quality studies were undertaken and are ongoing in Central California. Air quality research in the southern San Joaquin Valley commenced in the early 1980's (Blumenthal et al. 1985). The SARMAP modeling system was produced by SJVAQS and Atmospheric Utility Signatures, Predictions, and Experiments (AUSPEX) studies. A clear and parsimonious description of these studies and their relation to the SARMAP modeling system, later named SAQM, is provided by the SJVUAPCD:

The District's ozone model, which is currently being tested and used for attainment demonstration modeling, was developed in partnership with the 
EPA, state and local government, and industry. Many parties that contributed funds have participated in directing a study of an appropriate ozone event in the development a photochemical grid model appropriate for the San Joaquin Valley. The development of the model was funded by agreement between the San Joaquin Valley Air Quality Study (SJVAQS) and Atmospheric Utility Signatures, Predictions, and Experiments (AUSPEX). This modeling project was named SARMAP (SJVAQS/AUSPEX Regional Modeling Adaptation Project) and the model was subsequently named SAQM (SARMAP Air Quality Model). The model was developed in accordance with the EPA and the CARB modeling guidelines, and with the active participation of both agencies (SJVUAPCD 1994, page 1-6).

The SJVAQS (1996) was an 8-year, \$18 million research effort with the following policy objectives:

- Provide an improved understanding of the causes of high ozone in the Valley.

- Provide decision-makers with effective tools for identifying sound and equitable control strategies to attain ambient air quality ozone standards.

In addition, the study had eleven technical objectives, including to understand:

- what types of weather patterns lead to high ozone in the Valley

- the extent to which pollutants from outside the Valley contribute to high ozone in the Valley

- pollutant movement within the Valley

- if emissions in the Valley contribute to high ozone levels elsewhere

- what sources of emissions lead to ozone formation

- how ozone-causing emissions sources are distributed through the Valley

- how much natural emissions influence ozone formation

- the chemical processes that lead to ozone formation

- what chemical processes limit ozone formation or remove it from the atmosphere 
- if additional reductions of hydrocarbon emissions, $\mathrm{NO}_{\mathrm{x}}$ emissions, or both would be most beneficial for reduction ozone concentrations in the Valley

- how much emissions must be reduced to attain the state and federal ozone standards in the Valley

In addition to the SARMAP modeling system development, the SJVAQS funded technical research and a 1990 field study. The episode observed in early August, 1990, is the primary episode analyzed using modeling for ozone air quality planning in the SJV. It was the episode simulated for the 94 and Draft 02 Ozone Plans.

\section{Central California Ozone Study}

The Central California Ozone Study (CCOS) is the follow-up to the SJVAQS. Three levels of effort were proposed, which would total nearly \$10 million if fully funded. Funding from the CARB was committed to support the minimum level of effort. CCOS involves four efforts: (1) field study conducted in 2000, (2) data analysis, (3) emissions inventory updates, and (4) modeling. The CCOS effort led to the updates of the emissions inventory discussed earlier in this chapter. In addition, CCOS is intended to provide the capability to conduct modeling simulations in support of planning to meet the new federal ozone standard based on an 8-hour averaging time (CCOS 1999).

\section{California Regional Particulate Air Quality Study}

Ongoing and concurrent with the ozone studies is the California Regional Particulate Air Quality Study (CRPAQS). The study objectives are to:

- provide an improved understanding of emissions, $\mathrm{PM}_{10}$ and $\mathrm{PM}_{2.5}$ composition, and dynamic atmospheric processes

- establish strong scientific foundation for informed decision-making 
- develop methods to identify the most efficient and cost-effective emission control strategies to achieve the $\mathrm{PM}_{10}$ and $\mathrm{PM}_{2.5}$ standards in Central

California (CARB 2001d)

The \$27.5 million dollar project included multi-year, intensive field studies in 1995

and from 1999 through 2001, as well as data analysis and modeling to plan for attainment of federal PM standards.

The Central California Air Quality Studies, which include SJVAQS, AUSPEX, CRPAQS and CCOS, are multi-agency, multi-stakeholder efforts with local, state, federal and private sponsors. Sponsors are listed in Appendix IV-A.

\section{Summary and Conclusions}

This chapter surveys air quality conditions in Central California in terms of ozone and particulate matter. The complexity of air quality, as well as the limitations of observations, makes it difficult to evaluate conditions and trends conclusively. Air districts depict progress even though some data for the past decade suggest that ozone and particulate conditions have not improved very much. Estimates of per capita and area-weighted exposure to ozone in the SJV do show declines since 1990. The following are important conclusions from this chapter:

- Ozone and particulate conditions are far worse in the SJV than the Bay Area. The ozone and PM NAAQS are violated quite often in the SJV, and less frequently in the Bay Area. Air quality trends over the past 10 years show little significant decline in the number of violation days; EPA finds no significant trend (EPA 2001). SJVUAPCD and BAAQMD official designations are shown in Table IV-F. 
Table IV-F: BAAQMD and SJVUAPCD Air Quality Status

\begin{tabular}{|c|c|c|}
\hline Pollutant (Standard) & SJVUAPCD & BAAQMD \\
\hline $\begin{array}{l}\text { Federal 1-Hour Ozone } \\
\text { (No more than three days averaged over any three-year } \\
\text { period when peak ozone exceeds } 120 \text { ppb for one hour.) }\end{array}$ & $\begin{array}{c}\text { Severe } \\
\text { Nonattainment }\end{array}$ & $\begin{array}{c}\text { Unclassified } \\
\text { Nonattainment }\end{array}$ \\
\hline $\begin{array}{l}\text { State 1-Hour Ozone } \\
\text { (No more than three days averaged over an three-year } \\
\text { period when peak ozone exceeds exceeds } 90 \mathrm{ppb} \text { for one } \\
\text { hour.) }\end{array}$ & $\begin{array}{c}\text { Severe } \\
\text { Nonattainment }\end{array}$ & $\begin{array}{c}\text { Severe } \\
\text { Nonattainment }\end{array}$ \\
\hline $\begin{array}{l}\text { Federal } \mathrm{PM}_{10} \\
\text { (Annual arithmetic mean less than } 50 \mu \mathrm{g} / \mathrm{m}^{3} \text { and 24-hour } \\
\text { average less than } 150 \mu \mathrm{g} / \mathrm{m}^{3} \text { ) }\end{array}$ & $\begin{array}{c}\text { Serious } \\
\text { Nonattainment }\end{array}$ & Compliant \\
\hline
\end{tabular}

- The science of the ozone and particulate problems is quite complex and interrelated. Emissions sources of PM vary with season and the meteorology. Although highly variable, poor air quality can occur throughout the year.

- Estimates of emissions are ever improving but still quite uncertain.

- Ozone and particulate air pollution are related to each other. So too are criteria pollutants, visibility and exposure to toxic air pollutants. Hydrocarbon and nitrogen oxides emissions are precursors to ozone formation. Nitrogen oxides can also form fine particulate matter as ammonium nitrate. Particulate concentrations impact visibility directly.

- New standards promulgated by the U.S. EPA for peak ozone averaged over 8hours and particulate matter smaller than 2.5 microns in diameter will represent new planning challenges, and will increase the impetus to control non-point emissions sources. Mobile and area sources are likely to be implicated increasingly for poor air quality in the SJV. 


\section{E. Appendix IV-A: Central California Air Quality Study Sponsors}

\begin{tabular}{|c|c|c|}
\hline Sponsor & SJVAQS & CRPAQS \\
\hline \multicolumn{3}{|l|}{ Federal Agencies } \\
\hline U.S. EPA & $\sqrt{ }$ & $\sqrt{ }$ \\
\hline U.S. Dept of Transportation & & $\sqrt{ }$ \\
\hline U.S. Dept of Agriculture & & $\sqrt{ }$ \\
\hline U.S. Dept of Navy & & $\sqrt{ }$ \\
\hline U.S. Dept of Defense & $\sqrt{ }$ & $\sqrt{ }$ \\
\hline U.S. Dept of Interior, Forest Service & $\sqrt{ }$ & $\sqrt{ }$ \\
\hline \multicolumn{3}{|l|}{ Local Government Agencies } \\
\hline California Air Resources Board & $\sqrt{ }$ & $\sqrt{ }$ \\
\hline California Energy Comm. & $\sqrt{ }$ & \\
\hline SJVUAPCD & $\sqrt{ }$ & $\sqrt{ }$ \\
\hline BAAQMD & $\sqrt{ }$ & $\sqrt{ }$ \\
\hline Sacramento County APCD & & $\sqrt{ }$ \\
\hline Kern County APCD & $\sqrt{ }$ & \\
\hline Stanislaus Area Assoc of Governments & $\sqrt{ }$ & \\
\hline Bakersfield High School & & $\sqrt{ }$ \\
\hline \multicolumn{3}{|l|}{ Cities } \\
\hline Bakersfield & $\sqrt{ }$ & \\
\hline Ceres & $\sqrt{ }$ & \\
\hline Clovis & $\sqrt{ }$ & \\
\hline Corcoran & $\sqrt{ }$ & \\
\hline Fresno & $\sqrt{ }$ & \\
\hline Hanford & $\sqrt{ }$ & \\
\hline Hughson & $\sqrt{ }$ & \\
\hline Huron & & $\sqrt{ }$ \\
\hline Kettleman City & $\sqrt{ }$ & \\
\hline Kingsburg & & $\sqrt{ }$ \\
\hline Lemoore & $\sqrt{ }$ & \\
\hline Manteca & $\sqrt{ }$ & \\
\hline Modesto & $\sqrt{ }$ & \\
\hline Newman & $\sqrt{ }$ & \\
\hline Oakdale & $\sqrt{ }$ & \\
\hline Patterson & $\sqrt{ }$ & \\
\hline Riverbank & $\sqrt{ }$ & \\
\hline Sanger & $\sqrt{ }$ & \\
\hline Taft & & $\sqrt{ }$ \\
\hline Turlock & $\sqrt{ }$ & \\
\hline Visalia & $\sqrt{ }$ & \\
\hline Wasco & & $\sqrt{ }$ \\
\hline Waterford & $\sqrt{ }$ & \\
\hline \multicolumn{3}{|l|}{ Counties } \\
\hline Fresno & $\sqrt{ }$ & \\
\hline \multicolumn{3}{|l|}{ Kings } \\
\hline \multicolumn{3}{|l|}{ Madera } \\
\hline \multicolumn{3}{|l|}{ San Joaquin } \\
\hline Stanislaus & & \\
\hline
\end{tabular}




\begin{tabular}{|c|c|c|}
\hline Sponsor & SJVAQS & CRPAQS \\
\hline \multicolumn{3}{|l|}{ Tulare } \\
\hline \multicolumn{3}{|l|}{ Industrial Associations } \\
\hline Atlantic Richfield Co. & $\sqrt{ }$ & \\
\hline Basic Amer. Foods & $\sqrt{ }$ & \\
\hline Beard Land Improvement Co. & $\sqrt{ }$ & \\
\hline \multicolumn{3}{|l|}{ Building Assoc. of CA } \\
\hline CA Cattleman’s Association & & $\sqrt{ }$ \\
\hline CA Cotton Ginner/Grower Assoc. & & $\sqrt{ }$ \\
\hline CA Farm Bureau Federation & $\sqrt{ }$ & $\sqrt{ }$ \\
\hline Chemical Waste Mgmt. & $\sqrt{ }$ & \\
\hline Chevron USA & $\sqrt{ }$ & \\
\hline Del Monte Corp. & $\sqrt{ }$ & \\
\hline E\&J Gallo Winery & $\sqrt{ }$ & \\
\hline EPRI & $\sqrt{ }$ & \\
\hline Foster Poultry Farms & $\sqrt{ }$ & \\
\hline Fresno County Farm Bureau & $\sqrt{ }$ & \\
\hline GWF Power Systems & $\sqrt{ }$ & \\
\hline Independent Oil Producers Agency & $\sqrt{ }$ & $\sqrt{ }$ \\
\hline Kern County Farm Bureau & $\sqrt{ }$ & \\
\hline Kings County Farm Bureau & $\sqrt{ }$ & \\
\hline Merced County Farm Bureau & & $\sqrt{ }$ \\
\hline Morning Star Packing Co. & $\sqrt{ }$ & \\
\hline Nisei Farmers League & & $\sqrt{ }$ \\
\hline Occidental International & $\sqrt{ }$ & \\
\hline Ogden Martin Systems & $\sqrt{ }$ & \\
\hline Oxford Energy Inc. & $\sqrt{ }$ & \\
\hline PG\&E & $\sqrt{ }$ & \\
\hline PSE & $\sqrt{ }$ & \\
\hline San Joaquin Farm Bureau & $\sqrt{ }$ & \\
\hline Shell Western E\&P & $\sqrt{ }$ & \\
\hline Southern CA Gas Company & $\sqrt{ }$ & $\sqrt{ }$ \\
\hline Stanislaus County Farm Bureau & $\sqrt{ }$ & \\
\hline Stanislaus Farm Supply Company & $\sqrt{ }$ & \\
\hline Texaco Inc. & $\sqrt{ }$ & \\
\hline Fri Valley Growers & $\sqrt{ }$ & \\
\hline Ultrapower Malaga & $\sqrt{ }$ & \\
\hline Western States Petroleum Assoc. & $\sqrt{ }$ & $\sqrt{ }$ \\
\hline Western United Dairymen & & $\sqrt{ }$ \\
\hline World Wide Sires Inc. & $\sqrt{ }$ & \\
\hline
\end{tabular}




\section{CENTRAL CALIFORNIA AIR QUALITY MODELING}

\section{A. Introduction}

This chapter describes modeling conducted to study air quality in Central California. Developed in the early 1990's as part of the SJVAQS, the SARMAP modeling system was applied to support ozone planning by the SJVUAPCD and to assess the transport of emissions from the Bay Area to the SJV. Discussed are details of SARMAP and its uncertainties when applied to the Aug. 2-6, 1990 ozone episode. The response to uncertainties by modelers and decision-makers is then critiqued to highlight how uncertainties are treated.

\section{B. SARMAP Modeling System}

Funding for SARMAP development was provided by SVJAQS and AUSPEX, which are described in the previous chapter. The model building effort was the SJVAQS/AUSPEX Regional Modeling Adaptation Project (SARMAP); the resultant model is SAQM (SARMAP Air Quality Model). Model simulations were supported by intensive field studies that produced one of the most extensive observational databases available at the time (CARB 1996b).

Two documents are the focus of what is presented in this chapter:

- The Ozone Attainment Plan finalized by the San Joaquin Valley Unified Air Pollution Control District in November, 1994 (referred to as 94 Ozone Plan)

- Performance Evaluation of SAQM in Central California and Attainment Demonstration for the August 3-6, 1990 Ozone Episode published by DeMassa et al. in CARB's Planning and Technical Support Division in February, 1996 (referred to as SARMAP Performance Evaluation) 


\section{SARMAP System Components}

SAQM is a three-dimensional model that simulates transport, dry deposition and chemical transformation (CARB 1996b). It is an updated version of Regional Acid Deposition Model (RADM), which was developed to study acid precipitation as part of the National Acid Precipitation Program (Cowling 1992) ${ }^{68}$. The SAQM system is shown in Figure V-1.

Figure V-1: SARMAP Modeling System

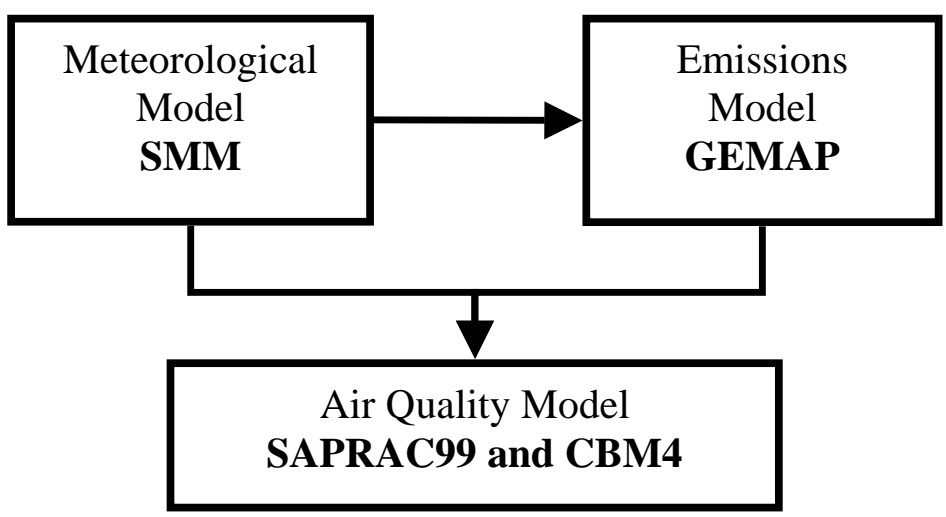

The meteorological model is SMM (SARMAP Meteorological Model), a version of the Mesoscale Meteorological v5 (MM5). MM5 is the latest version of a model developed, improved and widely applied over 20 years through a cooperative effort by Penn State University and the National Center for Atmospheric Research. It uses primitive conservation equations to predict: (1) wind speed and direction in three dimensions, (2) temperature, (3) mixing depth (i.e., heights of the inversion layer), (4)

\footnotetext{
${ }^{68}$ Improvements to SAQM include:

- $\quad$ a new mass conservation module to be compatible with inputs from the meteorological model

- $\quad$ an updated advection scheme to reduce artificial numerical dispersion

- $\quad$ a dry deposition module based on findings of the 1991 San Joaquin Valley Deposition Study

- $\quad$ the use of two chemical mechanisms, Carbon Bond Mechanism IV(CBM4) and Statewide Air Pollution Research Center (SAPRAC99), to replace the RADM2 mechanism.
} 
cloud cover, (5) precipitation and (6) the vertical diffusivity of heat and momentum. It also references observations iteratively to assure that predictions do not deviate too much from what was really observed. ${ }^{69}$

The Emissions Modeling System is an enhancement of Geocoded Emissions Modeling and Projection System (GEMAP). EMS applied for SARMAP is referred to as SEIM (SARMAP Emissions Inventory Model). Using ARC/INFO geographic information systems software, it accepts inputs resolved in time and the three dimensions of space produced by the meteorological model and source-specific emissions models. It uses the meteorological information to generate estimates of emissions from sources like motor vehicles and vegetation. SEIM also processes emissions estimates for point and area sources.

\section{Domain and Structure}

The SARMAP modeling domain includes the region described in Appendix $C$ Central CA Socioeconomic Setting, and shown in Figure APPC-2. It includes the San Francisco Bay Area, Sacramento metropolitan area, and the San Joaquin Valley. The domain is divided vertically by 30 layers that rise to $14 \mathrm{~km}$ above the surface. Horizontally, there are three nested grids of increasing resolution: 36 x $36 \mathrm{~km}^{2}, 12$ x 12 $\mathrm{km}^{2}$ and $4 \times 4 \mathrm{~km}^{2}$. The $12 \times 12 \mathrm{~km}^{2}$ resolution is the modeling domain shown in Figure APPB-2 of Appendix B. The $4 \times 4 \mathrm{~km}^{2}$ grids cover three areas that encompass Sacramento and San Francisco, Fresno, and Bakersfield, respectively. One modeling challenge is the alignment of mapping and gridding systems used by the air quality, meteorological and various emissions models. CARB does an admirable job of tracking

\footnotetext{
${ }^{69}$ The method is called four dimensional data assimilation (4DDA).
} 
it all, as detailed in (CARB 1996b), but disparities and errors still occur and contribute to uncertainties in modeling.

The ozone episode modeled was a five day period, August $2-6,1990$, when warm, stagnant conditions allowed pollutant concentrations to build until peak ozone concentrations of nearly $160 \mathrm{ppb}$ were observed. Domain-wide, ozone peaks were observed in and near Fresno and Bakersfield on the last two days of the event.

\section{SARMAP Uncertainties}

The SARMAP model has uncertainties associated with model formulation, inputs, and stochasticity. ${ }^{70}$ To illustrate these uncertainties, the SARMAP application simulated the Aug. 2-6, 1990 ozone episode observed in Central California is considered because it was used for (1) planning ozone NAAQS attainment in the SJV, (2) assessing pollutant transport from the Bay Area to the SJV, and (3) estimating the effects of Bay Area refinery $\mathrm{NO}_{\mathrm{x}}$ controls on local and downwind air quality. It has also been used extensively for research, but emphasis here is on its regulatory use.

\section{1994 Ozone Plan}

The SJVUAPCD and CARB describe several uncertainties directly and clearly in the 94 Ozone Plan and SARMAP Performance Evaluation. Several technical issues regarding SARMAP are discussed in Chapter 5 of the 94 Ozone Plan:

- The model is an approximation that cannot contain "all factors which appear in the real world...predictions made are only as precise as current science, technology, and information allow.” (Page 5-7)

\footnotetext{
${ }^{70}$ The fourth type of uncertainty - using modeling results in social decision-making processes - is the topic of the Chapters VI-IX.
} 
- Only one meteorological event is represented by the model application, but the weather is believed to be typical. However, atypical were the large number of ongoing forest fires. Analysis by CARB concluded that forest fires did not have a significant influence on ozone.

- Emissions from motor vehicles are "known to be underestimated" (page 5-8). Emissions are concentrated, incorrectly, along highway corridors, but that may have offset underestimates of vehicle emissions factors.

CARB and the SJVUAPCD listed some modeling uncertainties separately in the 94

Ozone Plan. CARB’s “caveats” were:

- There is "some concern over the spatial apportionment of motor vehicle emissions" (page 5-8) because it was based on a coarse, statewide model.

- The wind field around Fresno in the afternoon of August 6 is "known to be incorrect” (page 5-8).

- Estimates of winds and mixing heights are undergoing improvement.

- The 12 x $12 \mathrm{~km}^{2}$ wind field is based on sampling every third point of the $4 \mathrm{x}$ $4 \mathrm{~km}^{2}$ wind field with unclear consequences on model predictions.

- The 1999 emissions estimate was based on scaling from the 1990 baseline estimate, and did not include emissions reductions anticipated from the enhanced vehicle inspection and maintenance program.

The SJVUAPCD’s concerns were myriad. At issue was the temporal disconnect between model testing and the need to proceed with planning. Uncertainties not yet evaluated at the time of the 94 Ozone Plan production created a two-part concern. First, outstanding questions about the model were yet unexamined. Second, planning was forced to proceed with a model application that had not been tested to the satisfaction of the modelers. Other issues noted by the SJVUAPCD in the 94 Ozone Plan were: 


\section{Boundary Conditions}

The choice of pollutant concentrations blowing into the modeling domain from the west involved considerable deliberation. It was not defensible to use EPA's default values for a “clean air” boundary (e.g., VOCs = $18.8 \mathrm{ppb}$ at the surface) because the domain’s western boundary was influenced by offshore breezes containing inland emissions. Therefore aircraft were used to collect data from the area, which resulted in values used that were three times higher than EPA default values (e.g., VOCs $=51.5 \mathrm{ppb}$ at the surface). However, those concentrations were believed to be too high to represent diurnal fluctuations because they were collected in the morning when the mixing height was low. Furthermore, model formulation did not allow for diurnal or horizontal variation along the boundary. When modeled ozone concentrations showed "resistance to emissions changes", the SJVUAPCD expressed concern it was due to "excessive boundary condition loading” (SJVUAPCD 1994).

\section{Prediction Accuracy}

Although overestimated relative to observed levels, ozone concentrations predicted by SARMAP met the established performance criteria. The overestimates occurred despite emissions estimates that were known to be low. This suggested the presence of compensating error in the model, since too few emissions should cause the model to underestimate ozone formation. The boundary conditions discussed above were the probable culprit (SJVUAPCD 1994). 


\section{Model Sensitivity}

The simulations produced "small or no change in ozone predictions in response to changes in the emissions” (SJVUAPCD 1994). This apparent lack of sensitivity may be due to erroneous boundary conditions, as just discussed.

\section{Additional Model Uncertainties}

Noting "uncertainties in the model and the limited spatial accuracy of point and mobile sources", the SJVUAPCD concluded that "the reductions indicated as necessary to reach attainment may be significantly overestimated" and that "attainment could be reached by balanced domain wide $\mathrm{VOC}$ and $\mathrm{NO}_{\mathrm{x}}$ reductions of 30 percent, or $\mathrm{NO}_{\mathrm{x}}$ reductions alone of 36 percent” (SJVUAPCD 1994).

\section{Quality Assurance}

The process of assuring the quality of data gathered during the SJVAQS field program was ongoing at the time of the 94 Ozone Plan production.

\section{SARMAP Performance Evaluation}

Several issues not noted in the 94 Ozone Plan are addressed by CARB in its SARMAP Performance Evaluation (CARB 1996b). Indeed, CARB's technicians were tenacious in their examination of SARMAP and their pursuit of uncertainties. Much of their analyses took place after the 1994 planning cycle. The performance evaluation was guided in part by suggestions from the Technical Committee (Roth et al. 1998). Detailed in the SARMAP Performance Evaluation are:

- Methods used to map and track input data in the modeling domain.

- Locations of aircraft and surface monitoring, and data gathered. 
- Conceptual descriptions of the episode as it evolved from Aug. 3 through Aug. 6, including climatology, meteorology and air quality.

- The evolution of and rational for successive generations of initial conditions, boundary conditions, and emissions estimates. Presumably, each new generation was an improvement over the prior estimate.

- Standard performance statistics summarizing differences between observed and predicted peak ozone

- Performance statistics for ozone precursors, indicator and long-lived pollutants, including as $\mathrm{NO}, \mathrm{NO}_{2}, \mathrm{CO}$, Non-methane Hydrocarbons (NMHC), and peroxyactyl nitrate (PAN).

- Investigative simulations, which were actually sensitivity studies that estimated the change in peak modeled ozone due to changes in inputs, such as domain-wide emissions, boundary and initial conditions, biogenic emissions, and deposition.

The SARMAP Performance Evaluation report documents model development, methods, and assumptions, and model performance, and presents simulations used to test the sensitivity of the model to changes in inputs. It provides insight into how modelers grapple with alternative input values and assumptions, and the implications of modeling decisions on model results and sensitivities. Investigative simulations reported in the SARMAP Performance Evaluation are summarized in Table V-A. The simulations that predicted peak ozone was sensitive to emissions estimates, boundary conditions and the treatment of deposition. 
Table V-A: CARB's Investigative Simulations with SARMAP ${ }^{71}$ (CARB 1996b)

\begin{tabular}{|l|c|c|c|}
\hline Simulation & Domain Peak Ozone & $\begin{array}{l}\text { Difference from Base } \\
\text { Case (ppb) }\end{array}$ & $\begin{array}{l}\text { Difference from Base } \\
\text { Case (\%) }\end{array}$ \\
\hline Base Case & $14.2 \mathrm{ppb}$ & $\mathrm{N} / \mathrm{A}$ & N/A \\
\hline Zero Emissions & 8.9 & -5.3 & $-37 \%$ \\
\hline $\begin{array}{l}\text { Zero Boundary and } \\
\text { Initial Conditions }\end{array}$ & 9.4 & -4.8 & $-4 \%$ \\
\hline $\begin{array}{l}\text { “Clean Air” Boundary } \\
\text { and Initial Conditions }\end{array}$ & 13.7 & -0.5 & $6 \%$ \\
\hline $\begin{array}{l}\text { Increased Boundary and } \\
\text { Initial Conditions }\end{array}$ & 15.0 & 0.8 & $-15 \%$ \\
\hline $\begin{array}{l}\text { Zero Biogenic } \\
\text { Emissions }\end{array}$ & 12.1 & -2.1 & $23 \%$ \\
\hline Zero Deposition & 17.5 & 3.3 & \\
\hline
\end{tabular}

\section{Additional Uncertainties}

In terms of model inputs, boundary values and some emissions estimates are

discussed in both the 94 Ozone Plan and the SARMAP Performance Evaluation. Several uncertainty issues, however, were not addressed in either document, including:

\section{Reality Context}

There are differences between observed peak, modeled peak, and design values for ozone. Peak ozone observed during the Aug. 2-6 episode was $160 \mathrm{ppb}$, which is $10 \mathrm{ppb}$ lower than the design value of $170 \mathrm{ppb}^{72}$ Peak ozone predicted by SARMAP was 152 ppb on Aug. 5 (near Fresno) and 158 ppb on Aug. 6 (near Bakersfield). Thus, peak ozone concentrations modeled were $12 \mathrm{ppb}$ below the design value. The peak ozone concentration predicted in the base case simulations by CARB in their SARMAP Performance Evaluation was 142 ppb, which was 16\% below the design value and 11\%

\footnotetext{
${ }^{71}$ The SARMAP Performance Evaluation reports results of investigative simulations for August 5 and 6, and for the southern, central and northern areas of the domain. Reported here are the domain-wide peaks for August 6.

${ }^{72}$ The design value is the fourth highest 1-hour ozone concentration measured in any three-year period. The form of the ozone NAAQS allows for an average of one annual violation of the concentration
} 
below the peak observed concentration on Aug. 6, 1990. Planning for a $40 \mathrm{ppb}$ decrease (160 ppb down to $120 \mathrm{ppb}$ ) in peak ozone does not assure enough change to meet the standard, since ozone on the design value day exceeded the standard by $50 \mathrm{ppb}$.

\section{Representativeness}

Although CARB notes that the modeled episode was "typical" except for forest fires, it was not actually representative of the myriad conditions contributing to high ozone concentrations. The SJVUAPCD acknowledged this concern:

Since weather conditions vary, it is desirable to analyze more than one weather pattern which lead to a high ozone level. A possibility exists that the effect of controls may vary under different weather conditions and the best control plans would be successful under any weather conditions which could cause high ozone levels" (94 Ozone Plan, page 3-4).

Though the quote expresses optimism, there is also a possibility that different weather conditions will not be sufficiently responsive to control plans.

\section{Ongoing Performance Evaluation}

Although the 94 Ozone Plan notes that model development and testing is ongoing, it says "model improvements may affect the intensity of response to an emissions change but are not likely to alter conclusions to a significant degree” (page 2-11). Furthermore, the plan indicates that uncertainties may mean that "reductions indicated as necessary to reach attainment may be significantly overestimated” (page 6-3). In hindsight, we know that the opposite proved true. 


\section{Emissions Estimates (Revisited)}

The emissions estimate was a source of controversy, as it was evolving up to the application of SARMAP for planning purposes. Known emissions sources, notably nonroad vehicles, livestock waste and agricultural equipment, were not quantified in the emissions inventory because estimation methods were not yet approved. CARB and the SJVUAPCD were forthright in their treatment of these concerns, but ultimately had to use the "best available" estimate. Nonetheless, there was information available about the extent of uncertainty associated with quantified biogenic and motor vehicle emissions that was not taken into account in planning.

\section{Motor Vehicle Emissions}

The motor vehicle emissions estimate was noted to be wrong in 94 Ozone Plan (SJVUAPCD 1994, page 5-8). It was well documented to be underestimated by approximately a factor of two. Independent research using fuel-based and remote sensing approaches did not corroborate the standard method used to generate the estimate used for modeling (Singer and Harley 1996). That method relies on an estimate of motor vehicles, their use rates, and emissions rates per vehicle. Regulatory requirements prohibited revising the motor vehicle emissions estimate based on results of other methods. Time for plan submission was running short, so updates were administratively prohibited. For example, the public must be given an opportunity to comment on changes to the estimate of emissions. Furthermore, other planning documents, such as the Rate of Progress reports, which was included as Chapter 8 of the 94 Ozone Plan, and transportation planning necessitated the use of a consistent motor vehicle emissions estimate. 
To evaluate the implications of motor vehicle emissions estimate uncertainty, CARB executed SARMAP with motor vehicle emissions doubled. They concluded "ozone will be over-predicted with a motor vehicle increase with the existing boundary conditions" (CARB 1996b). This is no surprise since the model was predicting ozone close to observed level with the original, lower estimate of motor vehicle emissions. This finding, however, is not very informative because the sensitivity simulation skips important steps. Examining the sensitivity of peak ozone to a doubling of motor vehicle emissions is a large, not "local" change in pollutant load and therefore requires the following steps:

1. Double the motor vehicle emissions inventory

2. Adjust the boundary values or other sources of pollutant inputs downward to assure that the net loading of pollutants in the domain remains approximately similar to the original, base case simulation

3. Evaluate model performance with this new set of inputs

4. Conduct sensitivity studies on the motor vehicle emissions estimate using small (i.e., local) changes in the estimate

Simply adding a large increment of emissions to a simulation, while skipping steps 2 and 3, led CARB to a potentially spurious conclusion that the model would have predicted too much ozone with a more accurate estimate of vehicular emissions.

Similarly, as discussed later, future case scenarios incorporate an assumption that ozone boundary conditions would be lower due to emissions controls implemented upwind in the Bay Area and Sacramento air districts (CARB 1996b). The steps for validating the model with a new motor vehicle emissions estimate need also be taken for the boundary conditions assumed in the future case.

\section{Biogenic Emissions}


The estimate of VOCs emissions from vegetation is not discussed in the 94 Ozone Plan, except to note that biogenic emissions were included in the inventory. Biogenic emissions received limited treatment in the SARMAP Performance Evaluation. Nonetheless, they were a source of uncertainty.

Indicative of the uncertainty in 1994, the biogenic emissions estimate was halved in the updated subsequent estimate used for the Draft 02 Ozone Plan (SJVUAPCD 2001b). As shown in Table V-B, CARB conducted an experimental simulation that set biogenic emissions to zero and found peak modeled ozone declined from $14.2 \mathrm{ppb}$ to $12.1 \mathrm{ppb}$, a reduction of 15 percent. However, as discussed in the next section, the results must be questioned because the large modeled emissions change may have pushed the model beyond its range of validity.

The sources of uncertainty in biogenic emissions estimates are myriad. A regionalscale estimate will incorporate limited understanding of feedback processes (e.g., natural sinks for VOCs), plant physiological processes controlling VOC emissions, and reactions to environmental factors that may be unique to plant species or individual plants. Many biological and environmental factors may influence the rate of VOC emissions from an individual plant specimen. When generating an estimate of emissions for a modeling domain, the following components of the estimate are uncertain (Fine and Roth 1996):

- area coverage of vegetation community

- plant species composition of vegetation community (e.g., blue oak woodland)

- biomass estimate of plant species comprising vegetation community

- VOC emissions factors for plant species 
- emissions factors adjustments to account for environmental conditions (e.g., temperature, insolation)

- natural variability in each of the above factors

Uncertainties associated with the biogenic emissions estimated for SARMAP are provided by Tanner (1992), as shown in Table V-B. In reviewing model-based biogenic emissions estimates for California, Winer (1996) concluded that the uncertainty for each component (e.g., VLC, leaf biomass, species specific emissions factors, and environmental adjustment algorithms) of available models (e.g., BEIS, BEIS2, VEGIES, and GEMAP) was a factor of two.

Table V-B: Uncertainty in SARMAP Biogenic Emissions Estimates (Fine and Roth 1996 based on Tanner 1992)

\begin{tabular}{|c|c|c|c|}
\hline Variable & Uncertainty & Condition & Basis \\
\hline $\begin{array}{l}\text { Plant Community } \\
\text { Classification }\end{array}$ & $\pm 15 \%$ & $\begin{array}{l}\text { Where remote observations (e.g., satellite } \\
\text { images) are used for classification }\end{array}$ & $\begin{array}{l}\text { Remote sensing } \\
\text { experts }\end{array}$ \\
\hline \multirow[t]{3}{*}{$\begin{array}{l}\text { Biomass Factor } \\
\text { for Each Plant } \\
\text { Species }\end{array}$} & $\pm 20 \%$ & $\begin{array}{l}\text { Where plant species distribution was available } \\
\text { or total community biomass was surveyed }\end{array}$ & \multirow[t]{3}{*}{$\begin{array}{l}\text { Variability of } \\
\text { species measured } \\
\text { in region }\end{array}$} \\
\hline & $\pm 50 \%$ & $\begin{array}{l}\text { Where species distribution was available but } \\
\text { total biomass estimate was based on scaling to } \\
\text { another plant community }\end{array}$ & \\
\hline & $\pm 90 \%$ & $\begin{array}{l}\text { Where species distribution was not known and } \\
\text { total biomass estimate was based on scaling to } \\
\text { another community }\end{array}$ & \\
\hline \multirow[t]{4}{*}{ Emissions Factor } & $\pm 20 \%$ & $\begin{array}{l}\text { Where species emissions factor had been } \\
\text { measured }\end{array}$ & \multirow[t]{4}{*}{$\begin{array}{l}\text { Variability of } \\
\text { laboratory } \\
\text { measurements, } \\
\text { taxonomic } \\
\text { associations have } \\
\text { "tenuously } \\
\text { documented" } \\
\text { uncertainty }\end{array}$} \\
\hline & $\pm 40 \%$ & $\begin{array}{l}\text { Where an emissions was based on measurement } \\
\text { of emissions from a species in the same genus }\end{array}$ & \\
\hline & $\pm 60 \%$ & $\begin{array}{l}\text { Where an emissions was based on measurement } \\
\text { of emissions from a species in the same family }\end{array}$ & \\
\hline & $\pm 90 \%$ & $\begin{array}{l}\text { Emissions factor was based on an educated } \\
\text { guess }\end{array}$ & \\
\hline
\end{tabular}




\section{Model Sensitivity (Revisited)}

The investigative studies presented by CARB in the SARMAP Performance Evaluation (and summarized above in Table V-A) raises some methodological concerns. Standard performance statistics indicate how well the model simulates observed ozone for a nominal set of values. A "local" sensitivity test changes those values slightly to measure resultant changes in model outputs.

The sensitivities reported in the SARMAP Performance Evaluation were not estimates of local sensitivities, because the changes studied were quite significant (e.g., reducing emissions to zero rather than by ten percent). The approach used pushes the model beyond its limits of validity. The SARMAP Performance Evaluation noted that some of these assumptions might "adversely affect the chemistry” (page 103).

As reported, it appears that the investigative studies used major perturbations in the model. To do so in a manner that produces reliable results, the model must first be shown to be valid in the new region of operation. Effects on chemistry are a concern. However, no method is described in the documentation to indicate that these steps were taken.

Furthermore, another possible step, apparently not taken, may increase confidence in results. The signs and magnitudes of modeled responses to small changes in the original nominal values might be compared with sensitivities calculated with the new set (i.e., doubled motor vehicle emissions and adjusted boundary conditions) sensitivities. Where values remain consistent, it suggests the sensitivities might be observed through a range of input values. 
Another concern is that these studies were conducted even though large error and bias were calculated for many pollutant species. Standard performance statistics indicated that error and bias associated with ozone predictions were in the range of 20 and 10 percent, respectively. Error and bias for predicted $\mathrm{NO}, \mathrm{NO}_{2}, \mathrm{CO}$, NMHC and PAN were much higher. These statistics indicated the presence of compensating errors - that the model was generating accurate results for ozone, but doing so for the wrong reasons. How could it be that model simulated ozone concentrations were very close to observed values, but other modeled values that determine ozone concentration were quite different? This question was never satisfactorily addressed in either the 94 Ozone Plan or the SARMAP Performance Evaluation. Furthermore, CARB proceeded with investigative simulations before diagnosing and correcting the cause(s) of compensating error(s).

\section{1994 Ozone Plan Simulations}

The 94 Ozone Plan discusses a series of SARMAP simulations, as summarized in

Table V-C..$^{73}$

\footnotetext{
${ }^{73}$ Various combinations of VOCs and $\mathrm{NO}_{\mathrm{x}}$ reductions in addition to those listed in the table were simulated to create an SJV Domain Control Isopleth, which is reproduced in Chapters X and XI as the SJV Composite Isopleth. ${ }^{73}$ The isopleth indicated that attainment was possible with either (a) $47 \% \mathrm{NO}_{\mathrm{x}}$ reduction domain-wide without VOCs reductions beyond those already planned, or (b) 38 and $40 \% \mathrm{NO}_{\mathrm{x}}$ and VOCs reduction, respectively, domain-wide.
} 
Table V-C: SARMAP Simulations in the 94 Ozone Plan

\begin{tabular}{|c|c|c|c|}
\hline $\begin{array}{l}\text { Simulation } \\
\text { Scenario }\end{array}$ & What is tested & Purpose & Result \\
\hline 1990 Base Case & None & $\begin{array}{l}\text { Is the observed event (Aug. } \\
\text { 2-3) being simulated within } \\
\text { performance requirements } \\
\text { set by CARB and EPA. }\end{array}$ & $\begin{array}{l}\text { Simulation meets } \\
\text { performance criteria. }\end{array}$ \\
\hline 1999 Base Case & $\begin{array}{l}\text { Include effects of } \\
\text { controls adopted before } \\
1994 \text { and projected } \\
\text { growth }\end{array}$ & $\begin{array}{l}\text { Determine if controls } \\
\text { already planned by the } \\
\text { SJVUAPCD, CARB and } \\
\text { upwind AQMDs would be } \\
\text { sufficient to achieve the } \mathrm{O}_{3} \\
\text { NAAQS by } 1999 \text { in the } \mathrm{SJV} \\
\text { in light of anticipated } 33 \% \\
\text { growth in population. }\end{array}$ & $\begin{array}{l}\text { Additional controls are } \\
\text { needed beyond those } \\
\text { already planned to meet } \\
\mathrm{O}_{3} \text { NAAQS by } 1999 \text {. }\end{array}$ \\
\hline Interbasin Transport & $\begin{array}{l}\text { Reduce all man-made } \\
\text { emissions in the San } \\
\text { Francisco Bay Area and } \\
\text { Sacramento to zero. }\end{array}$ & $\begin{array}{l}\text { Compared with } 1990 \text { Base } \\
\text { Case, are SJV emissions } \\
\text { alone sufficient to cause } \mathrm{O}_{3} \\
\text { NAAQS violations? } \\
\text { Determine the contribution } \\
\text { of upwind emissions to SJV } \\
\text { ozone concentrations. }\end{array}$ & $\begin{array}{l}\text { Emissions in the SJV } \\
\text { are enough to cause } \mathrm{O}_{3} \\
\text { NAAQS violations. } \\
\text { Peak ozone in the } \\
\text { northern, central and } \\
\text { southern portions of the } \\
\text { SJV decline by 27, } 10 \\
\text { and } 7 \text { percent, } \\
\text { respectively. }\end{array}$ \\
\hline $\begin{array}{l}\text { 100\% VOCs } \\
\text { Reduction }\end{array}$ & $\begin{array}{l}\text { Reduce man-made } \\
\text { VOC emissions } \\
\text { domain-wide to zero }\end{array}$ & $\begin{array}{l}\text { Will total VOC reductions } \\
\text { be sufficient to meet } \mathrm{O}_{3} \\
\text { NAAQS? }\end{array}$ & $\begin{array}{l}\text { Ozone NAAQS cannot } \\
\text { be achieved by } \\
\text { eliminating all VOC } \\
\text { emissions. }\end{array}$ \\
\hline $\begin{array}{l}50 \% \text { VOCs and } \mathrm{NO}_{\mathrm{x}} \\
\text { reduction }\end{array}$ & $\begin{array}{l}\text { Reduce man-made } \\
\text { VOC and } \mathrm{NO}_{\mathrm{x}} \\
\text { emissions domain-wide } \\
\text { by } 50 \% .\end{array}$ & $\begin{array}{l}\text { Will VOC and } \mathrm{NO}_{\mathrm{x}} \\
\text { reductions be sufficient to } \\
\text { meet } \mathrm{O}_{3} \text { NAAQS? }\end{array}$ & $\begin{array}{l}\text { Ozone NAAQS cannot } \\
\text { be achieved by } \\
\text { reducing } 50 \% \text { each of } \\
\text { VOC and } \mathrm{NO}_{\mathrm{x}} \\
\text { emissions. }\end{array}$ \\
\hline $\begin{array}{l}75 \% \text { VOCs and } 50 \% \\
\mathrm{NO}_{x} \text { reduction }\end{array}$ & $\begin{array}{l}\text { Reduce man-made } \\
\text { VOC and } \mathrm{NO}_{\mathrm{x}} \\
\text { emissions domain-wide } \\
\text { by } 75 \text { and } 50 \% \text {, } \\
\text { respectively. }\end{array}$ & $\begin{array}{l}\text { Will extensive VOC } \\
\text { reductions, plus moderate } \\
\mathrm{NO}_{x} \text { reductions, be } \\
\text { sufficient to meet } \mathrm{O}_{3} \\
\text { NAAQS? }\end{array}$ & $\begin{array}{l}\text { Ozone NAAQS cannot } \\
\text { be achieved by } \\
\text { reducing } 75 \% \text { of VOC } \\
\text { and } 50 \% \text { of } \mathrm{NO}_{\mathrm{x}} \\
\text { emissions. }\end{array}$ \\
\hline $\begin{array}{l}50 \% \text { VOCs and } 65 \% \\
\mathrm{NO}_{\mathrm{x}} \text { reduction }\end{array}$ & $\begin{array}{l}\text { Reduce man-made } \\
\text { VOC and } \mathrm{NO}_{\mathrm{x}} \\
\text { emissions domain-wide } \\
\text { by } 50 \text { and } 65 \% \text {, } \\
\text { respectively. }\end{array}$ & $\begin{array}{l}\text { Will extensive } \mathrm{NO}_{\mathrm{x}} \\
\text { reductions, plus moderate } \\
\text { VOC reductions, be } \\
\text { sufficient to meet } \mathrm{O}_{3} \\
\text { NAAQS? }\end{array}$ & $\begin{array}{l}\text { Attainment is possible } \\
\text { by reducing } 50 \% \text { of } \\
\text { VOC and } 65 \% \text { of } \mathrm{NO}_{x} \\
\text { emissions. }\end{array}$ \\
\hline $\begin{array}{l}1999 \text { Proposed } \\
\text { Actions Attainment } \\
\text { Demonstration (with } \\
\text { new emissions } \\
\text { reductions from } \\
1994 \text { plan) }\end{array}$ & $\begin{array}{l}\text { Reduce man-made } \\
\text { VOC and } \mathrm{NO}_{\mathrm{x}} \\
\text { emissions in the SJV by } \\
32 \text { and } 37 \text { tons, } \\
\text { respectively, beyond } \\
\text { reductions from already } \\
\text { adopted rules. }\end{array}$ & $\begin{array}{l}\text { Are reductions beyond those } \\
\text { already planned sufficient to } \\
\text { meet } \mathrm{O}_{3} \text { NAAQS? }\end{array}$ & $\begin{array}{l}\text { Attainment is possible } \\
\text { with additional } \\
\text { reductions. }\end{array}$ \\
\hline
\end{tabular}




\section{Refinery $\mathrm{NO}_{\mathrm{x}}$ Controls in Western Kern County}

Two additional simulations deserves mention: (1) evaluation of the need for refinery $\mathrm{NO}_{\mathrm{x}}$ emissions reductions in western Kern County, and (2) a study of the sensitivity of ozone predictions to errors in the motor vehicle emissions estimate, as discussed above.

After finding available planned controls to be sufficient, at least one other scenario was executed. In a final simulation, the anticipated $\mathrm{NO}_{\mathrm{x}}$ reductions from oilfields in the areas west of Interstate 5 in Kern, Kings and Fresno Counties were not included. After the simulation showed that the ozone NAAQS would still be met, the SJVUAPCD concluded that the oilfield emissions reductions were "not needed to reach the federal ozone standard” (page 4-34). Consequently, the final plan does not call for implementation of rules 4305 and 4702 at west side oilfields.

\section{Motor Vehicle Emissions Estimate Uncertainty}

The 94 Ozone Plan acknowledged that MV emissions were known to be uncertain. Sensitivity studies were conducted to determine if ozone concentrations were sensitive to an underestimate of MV emissions. CARB executed SARMAP with a hypothetical doubling of MV emissions and found that ozone unresponsive.

However, as discussed above, the analysis skipped the important steps of (a) adjusting other pollutant input sources downward as necessary to assure total pollutant loading made physical sense, and (b) performing an set of evaluations to assure model validity. Having skipped these steps, the conclusion is not reliable. First the boundary conditions or another emissions source had to be adjusted downward and the model shown to perform adequately with the new inventory. Only then might calculated sensitivities ozone response to changes in the MV estimate - be trusted. 


\section{E. Management of Uncertainties}

Last, and of particular relevance to this research, is how the uncertainties associated with technical information were managed by the SJVUAPCD in their emissions control strategy and attainment demonstration, and how CARB dealt with them in their transport assessment and mitigation. This discussion identifies a disconnect between uncertainties and planning decisions. In particular, the attainment demonstration portion of the 94 Ozone Plan accounted for uncertainties incompletely. Similarly, the SARMAP Performance Evaluation did not go far enough, even though it went beyond regulatory requirements and was more rigorous than other assessments of PAQSM performance. In the transport assessment, CARB dealt with uncertainties by offering corroborative evidence, even though that evidence was less reliable than modeling.

\section{1994 Ozone Plan}

There was a lack of translation of the technical uncertainties to the decision context in the 94 Ozone Plan. Planning decisions did not deal with SARMAP uncertainties explicitly. Although much of the 94 Ozone Plan describes why the control strategy is uncertain (e.g., evolving nature of SARMAP and emissions estimates), the control strategy itself does little to address uncertainty. Nor does the modeled attainment demonstration.

The control strategy set forth provides for just enough emissions reductions to meet the ozone NAAQS, and not a ton more. The approach concurs with the SJVUAPCD's responsibility to identify needed, but not excessive, controls, since too much reduction would constitute an unnecessary burden on the regulated community. Jim Sweet, SJVUAPCD, said, 
We want the model to tell us what the heck we're supposed to do. To accomplish our mission, which is to clean up the air without doing more than we need to do, without doing anything that is inappropriate.

The general theme of the first part of the 94 Ozone Plan is that there is considerable uncertainty. The document opens with a Letter of Intent Concerning Dynamic and Evolving Content of the San Joaquin Valley Air Quality Attainment Plan (page vii). Signed by David Howekamp, Director of EPA Region IX Air and Toxics Division, James Boyd, Executive Officer of CARB, and David Crow, Executive Director of the SJVUAPCD, this letter makes several points pertaining to uncertainty:

- The 94 Ozone Plan "is a dynamic and flexible plan...based upon the best science and information available at the time..., and is subject to change as better information is developed.”

- The key tool used in developing the plan is SARMAP. Past modeling capabilities were inadequate to "answer complex questions which underlie the needed comparison of different control strategy options...”.

- Partners of the SJVAQS "banded together to develop a sophisticated modeling tool" that "has achieved the federal model performance standards required for use in developing” the 94 Ozone Plan.

- Model refinements and evaluation will continue with unknown impacts on planning issues.

- New information may require updates to the emissions inventory, the SAQM model, or "modification, elimination, additions, or substitutions of control measures based upon new information or data."

- Therefore the 94 Ozone Plan may be modified "to provide further progress toward and timely attainment of the ozone standard."

The letter is further evidence of the decision-makers' understanding of the significance of uncertainty, and a desire to explain the implications for the current planning effort. Disclaimers notwithstanding, however, the document does not actually plan for the uncertainty. 
Discussions of the uncertainties create the impression that, if anything, the control strategy goes too far. In hindsight, of course, just the opposite has proven to be true. Back in 1994, however, there was enough evidence to acknowledge the possibility that uncertainties create an overly optimistic picture of future air quality. ${ }^{74}$

In discussing concerns that SARMAP overestimated ozone, the SJVUAPCD wrote:

The model replicated the observed levels of ozone using an emissions inventory that is known to have under estimations and omissions. This means that the model produced too much ozone either from the emissions inventory or from other supporting inputs such as the boundary conditions....If the problem is not related to boundary conditions, but is related to the emissions inventory, then the model is overestimating the amount of ozone formed per ton of precursors produced. This would constitute a more severe problem challenging the validity of the basic model formulation. It would also have the effect of making the model predict future year ozone concentrations higher than it should (page 5-11).

These assertions are misleading. The first assertion is correct, since the model was indeed overestimating ozone formed per ton of precursor emitted. Unacknowledged was the fact that the error aids the planning task. Recall that the objective is to show that planned controls will cause needed decreases in peak ozone. If ozone production is overly sensitive to precursor emissions, it will also appear, incorrectly, to be overly responsive to emissions reductions. Therefore, modeled emissions reductions seem more effective than should be expected in reality due to known errors in the SARMAP application.

This possibility of overly optimistic model responses is neither mentioned nor addressed in the 94 Ozone Plan. It is also contrary to the statement at the end of the

\footnotetext{
${ }^{74}$ As evidence from interviews presented in subsequent chapters reveals, few individuals involved in the process felt, back in 1994, that the 94 Ozone Plan was likely to succeed in meeting ozone NAAQS by 1999. However, the document itself did not depict the doubts of those who produced it.
} 
above paragraph. Future year ozone concentrations may appear lower than they should be, again helping with the attainment demonstration, because emissions were estimated to decrease in the future thanks to already planned reductions. Inclusion of additional emissions reductions will make ozone concentrations appear erroneously low in the attainment demonstration.

A third way the statement is misleading is that the high boundary values facilitated the attainment demonstration. Commenting on the attainment demonstration simulation for the 94 Ozone Plan, CARB wrote in the SARMAP Performance Evaluation,

For this simulation the base year ozone boundary conditions were reduced to $40 \mathrm{ppb}$ at the surface and aloft to reflect lower ozone as a result of emission reductions in the state (page 106).

Boundary conditions were assumed to decline between 1990 and 1999. Given the apparently erroneous sensitivity of ozone to boundary conditions, the attainment demonstration was facilitated by the decision to assume future year boundary conditions were lower than the base case year. ${ }^{75}$

Both CARB and independent researchers examined the importance of boundary condition assumptions in SARMAP simulations. CARB's effort is detailed in their SARMAP Performance Evaluation, as discussed previously and summarized in Table VB. In a subsequent analysis, Dabdub et al. (1999) executed a slightly modified version of SARMAP with three boundary condition scenarios:

1. All boundary concentrations set to zero

\footnotetext{
${ }^{75}$ An excerpt from the long quote in Chapter IX: Cent CA AQ Planning Prt I, Mr. David Jones, SJVUAPCD, said, Initially, the modeling didn't show attainment. When we were contemplating what else we could do, the decision was made to change some of the boundary conditions and some of
} 


\section{Ozone boundary concentration set to zero}

3. VOCs and $\mathrm{NO}_{\mathrm{x}}$ boundary concentrations set to zero

Through the first simulation, they found a "significant effect exerted by the species boundary conditions on predicted ozone in the San Joaquin Valley” (Dabdub et al. 1999, page 2509). They also noted that using a $\mathrm{NO}_{\mathrm{x}}$ boundary value of $3 \mathrm{ppb}$ meant that the total mass of $\mathrm{NO}_{\mathrm{x}}$ flowing into the region is four times estimated $\mathrm{NO}_{\mathrm{x}}$ emissions in the modeling domain. Indeed, boundary conditions mattered. Although the SJVUAPCD noted the potential significant prediction errors due to erroneous boundary conditions and emissions inventory sensitivities, no contingencies were put in place to deal with it.

The 94 Ozone Plan contained a discussion of the hypothetical implications of overprediction by the model of $0.5 \mathrm{ppb}$ ozone. It concluded that, whereas attainment was predicted to be reachable with a 47 percent reduction of $\mathrm{NO}_{\mathrm{x}}$ emissions domain-wide, or 40 and 38 percent reductions of VOCs and $\mathrm{NO}_{\mathrm{x}}$ emissions, respectively, a $0.5 \mathrm{ppb}$ overestimate would reduce reductions needs to 36 percent of $\mathrm{NO}_{\mathrm{x}}$ emissions, or 30 percent each of $\mathrm{NO}_{\mathrm{x}}$ and VOCs (see Table 5-2, page 5-12 of the 94 Ozone Plan).

Whereas the examination of a hypothetical over prediction of ozone by the model was examined, no similar analysis was conducted to explore the implications of under prediction. Had the SJVUAPCD done so, they may have not felt compelled, despite the language of section 182(f) of the CAAA, to excuse west side oilfields from $\mathrm{NO}_{\mathrm{x}}$ controls. 


\section{SARMAP Performance Evaluation}

Although the ozone episode simulation by SARMAP met performance requirements, CARB modelers continued testing the system. The effort is notable and praiseworthy, as it showed a tireless pursuit of understanding. Suggestions for testing had been submitted by the SJVAQS Technical Committee (Roth et al. 1998). Eventually, many of those tests were conducted. When the 94 Ozone Plan was being written, testing was incomplete (94 Ozone Plan; page 5-14). In fact, the SARMAP Performance Evaluation was not published until February 1996, thirteen months after the 94 Ozone Plan was finalized. Nonetheless, CARB's continued effort demonstrates a commitment to uncertainty identification, diagnosis and correction.

The SARMAP Performance Evaluation presents several significant findings:

- SARMAP predicts observed ozone very well, with error and bias in the range of 10 and 5 percent, respectively.

- SARMAP simulates complex physical and chemical processes that where observed during the episode, including overnight storage of nitrogen species and ozone in aloft layers, the morning growth in pollutant concentrations, inversion layer formation, and wind patterns near complex terrain.

- SARMAP does a poor job of predicting observed concentrations of ozone precursors (i.e., $\mathrm{NO}, \mathrm{NO}_{2}, \mathrm{NMHC}$ ), carbon monoxide or peroxyacetyl nitrate (PAN), with errors and biases generally between 50 and 100 percent.

- SARMAP cannot simulate the eddy wind current observed in Fresno.

SARMAP did an admirable job of simulating some very complex processes, yet it did not excel in the more stressful assessments. Notably, estimated concentrations of precursors and long lived species, such as PAN, were significantly different from those observed, and the eddy current observed in Fresno never appeared in the simulation. CARB never discusses the implications of the missing eddy current or the large observed 
biases and errors. Failure to respond to these concerns may have been understandable given limited time, budgets, or other constraints, but proceeding with the investigative simulations without understanding the sources of compensating errors was not very informative.

In addition to correcting compensating errors before proceeding with sensitivity analyses, the modelers needed to translate their findings. They have the greatest understanding of the model and modeling uncertainties, so it is their responsibility to translate their findings into uncertainty information applicable to the decision context. In this case, that translation meant explaining how the phantom eddy current and the precursor inaccuracies may have undermined the reliability of the SJVUAPCD's attainment demonstration. No attempt is made by CARB to discuss this possibility in the SARMAP Performance Evaluation.

\section{Transport Assessment}

CARB used the same August, 1990, SARMAP application to evaluate the influence that emissions in the Bay Area and Sacramento have on air quality in the SJV. This is the Interbasin Transport scenario listed above in Table V-B. Primary uncertainties attending that simulation included:

- representativeness of the modeled episode

- effects on chemistry from setting emissions to zero

- the need to acknowledge differences between an aged (i.e., transported) air mass, and fresh (i.e., local) emissions

CARB dealt with these uncertainties in two ways in finding the Bay Area to be an “overwhelming” contributor to ozone concentration in the SJV (CARB 2001c). First, the 
official language of the finding was that the contribution occurred only at certain times, but not necessarily every time the SJV experienced high ozone.

The second approach was to corroborate the modeling results with methods used previously to evaluate transport relationships. Two methods employed were examination of wind patterns data and a comparison of the ratio of upwind emissions to downwind emissions. Unfortunately, the corroborating methods themselves are quite uncertain and limited in their applicability. Wind patterns may not be representative. Comparisons of emissions estimates do not take into account chemistry or deposition that become relevant over transported distances and time. Ironically, it was the limits of these less sophisticated methods that led the regulated community to urge CARB to use SAQM for evaluating transport (for examples, see Ziman 1993a; Ziman 1993b; Ziman 1996c; Wang 1993).

Just as no translation of SARMAP Performance Evaluation results was provided for policy-makers, the limitations of the SARMAP simulation evaluating transport have been lost over time. The modeled ozone changes when upwind emissions were set to zero 27, 10 and 7 percent reductions in the north, central and south of the SJV region - have become the standard numbers cited, often without the uncertainty disclaimers. For example, see the quote from the SJVUAPCD's website in Appendix B - Regulatory Setting. 


\section{F. Summary and Conclusions}

Notable findings from this chapter are:

- The 94 Ozone Plan demonstrates that modelers and planners formulating it were well aware of the limitations of the SARMAP application. However, their treatment of the uncertainties was incomplete and misleading. Known uncertainties are characterized, incorrectly, as creating the possibility that planned controls will be excessive. Important uncertainties, such as the biogenic emissions estimate and the representativeness of the modeled episode, receive limited attention. Furthermore, the attainment demonstration component of the plan - the part that shows planned emissions reductions will result in attainment of the ozone NAAQS by 1999 - neither accounts for nor attempts to manage uncertainties.

- The SARMAP Performance Evaluation produced by CARB provides an extensive assessment of uncertainties attending the SARMAP application used in support of the 94 Ozone Plan. In presenting analyses, CARB demonstrates a commitment to understanding the model and its uncertainties. Unfortunately, like the 94 Ozone Plan, the effort does not go far enough. It does not translate known uncertainties to the decision context. Although there are no regulatory requirements to do so, modelers have the best understanding of modeling uncertainties and, as such, are the only ones in position to bridge the gap between the technical presentation of performance evaluation results and the needs of policy-makers.

- Bridging the gap will not be easy. As discussed in Chapter II - PAQSM and Their Uncertainties, no method is available for synthesizing the results of disparate uncertainty assessments. Even if they wanted to do so, CARB modelers reached to edges of capability that has not yet provided a way to build the bridge called for in this chapter. 


\section{CENTRAL CALIFORNIA PLANNING PART I - DESCRIPTION}

\section{A. Introduction}

To understand how plans to meet ozone air quality standards are produced in California, it is not possible to simply go to a library or search websites at EPA and CARB. There is a lot of information is available in the public domain about the impetus for planning and the agencies involved, as well as the routine monitoring and intensive field studies to measure air quality, the computer models used to simulate it, and how such research provides scientific bases for plans. What one cannot read about, however, is how the agencies interact and exchange information, or how the public and regulated industries participate. Nor can one determine what goes into the models or how the models are actually used.

This chapter and the next two describe the process (Chapter IX) as it took place in the Bay Area and the SJV in the 1990's, explain the decision possibilities, debates, and actual decisions (Chapter X), and; describe how models and their associated uncertainties are used (Chapter XI). Attention is focused on the players, their routes of interaction, information exchanged, and the dominant views about air quality problems and appropriate responses.

The description provides the basis for exploring how technical information, notably the results of modeling, is used in the decision-making. By extension, this work examines the potential utility of additional information, notably the rigorous assessment of uncertainties associated with modeling. Presented at increasingly fine levels of detail, each component of description concludes with a discussion of the use of models and uncertainty. 
A discussion of the type of uncertainty information to be produced, as well as the feasibility of producing it, is presented in previous chapters. Those chapters also categorize and document uncertainties. Uncertainty has many sources, so information describing it may take many forms. One ideal form of the information would enable decision-makers to assess the probability of meeting ozone air quality goals when modeling simulations indicate planned controls will do so. Planning thus far has not taken this step for making decisions in the face of uncertainty. These chapters explore why not by pursuing the following research questions:

- How does the ozone air quality planning process work in practice?

- How are models actually used for ozone planning?

- How is uncertainty managed in planning? What uncertainty information is sought? Who seeks it? Who resists it? Why?

- What constrains air quality planning decisions? Are the limits of scientific understanding one of the constraints? If so, might information about uncertainties in modeling lessen this constraint?

- What additional model uncertainty information might be useful?

In addition to the research questions, the description and critique of an air quality planning process facilitates the understanding and participation, if so desired, of interested citizens. Moreover, once the process is understood, modified or alternative processes may be envisioned to help the process achieve its mandate for air quality while maintaining the ideals of participatory democracy.

\section{B. The Process}

In their study of fiscal impact modeling, Dutton and Kraemer (1985) examine the political perspectives about model-based decision-making. They aim to understand if 
model-based planning could be "objective, credible, and accurate.” They also explore if new modeling technology only "automates the partisan biases of politicians.” Two dimensions of this question - control of the modeling and interests served by the modeling - are summarized by Dutton and Kraemer in a matrix reproduced here as Figure VI-1. The matrix maps four perspectives - technocratic, rational, partisan and consensual - that describe modeling studies in terms of the degree of autonomy exerted by technical experts and the extent to which pluralistic views are incorporated.

When distinguishing these perspectives, Dutton and Kraemer define a modeling study as a three-stage process: introduction, adaptation, and incorporation. With the rational perspective, the incorporation of results into decisions is the most important stage of a modeling study because models provide "best estimates" and inform choice. Those critiquing model-based air quality planning (for examples, see Demerjian et al. 1995; NARSTO 2000a) offer prescriptions that treat the process as rational because they assume implicitly that better technical information, such as information about the uncertainties in modeling results, will lead to more successful air quality planning. According to Dutton and Kraemer (1985), the rational perspective has two components: (1) technical experts control the modeling, and; (2) pluralistic interests are served. 
Figure VI-1: Political Perspectives on Models

\begin{tabular}{|l|l|l|}
\hline $\begin{array}{l}\text { Dominant Locus } \\
\text { of Control }\end{array}$ & \multicolumn{1}{|c|}{ Dominant Interest Served } \\
& \multicolumn{1}{|c|}{ Partisan/Self-interest $\begin{array}{c}\text { Non-Partisan/ } \\
\text { Pluralistic Interests }\end{array}$} \\
\hline Technical Experts & Technocratic Perspective & Rational Perspective \\
\hline Political Elites & Partisan Perspective & Consensual Perspective \\
\hline
\end{tabular}

The technocratic perspective believes the purpose of modeling is to transfer the ideas of technical experts and that models reflect a "truth” associated with expert knowledge. It is articulated by Fisher (1990, page 17) when he defines technocracy as, “a system of governance in which technically trained experts rule by virtue of their specialized knowledge and position in dominant political and economic institutions”. This perspective assumes that policy choices will derive from experts' ideas, since the policies will be imbedded in the logic of the model. The difference between the technocratic and rational perspectives is the extent to which partisan or pluralistic interests are served. A technocratic modeling process will incorporate primarily the values and interests of technicians in charge, whereas a rational process still relies on technicians but nonpartisan values and interests are considered.

The partisan perspective believes "models are tools of propaganda and persuasion of special interests in the policy process” (Dutton and Kraemer 1985, page 7). In this view, models are used to legitimate and defend predetermined decisions, where decisionmaking follows a “decision-propaganda-conformity” path, instead of the rationalist's path 
of “problem-information-decision”. If air quality planning is a partisan process, then modeling will serve the interests of the dominant political coalitions.

Finally, the consensual perspective is that models are primarily tools for negotiation, bargaining and interactive decision-making among conflicting interests and opinions. Dutton and Kraemer (1985) say this perspective emerged from their research. They note that consensus is not necessarily the objective of the process. Rather, it is the defining and conducting of the modeling study that is most important; model results are secondary considerations.

Which of these perspectives best describes modeling for air quality planning? The SARMAP modeling was the product of the San Joaquin Valley Air Quality Study, a “cooperative” study conducted by government officials and industry representatives (SJVAQS 1996). The SJVAQS used the management structure shown below in Figure VI-2 (SJVAQS 1996). The Study Agency is a legal entity established to manage funds for the SJVAQS and decision-making was described as (SJVAQS 1996),

The study's participants generally reached agreement by consensus. When an issue needed to be resolved by voting, each of the four interest blocks the federal government, State of California, local governments, and private sector - was given one vote. 
Figure VI-2: SJVAQS Management Structure

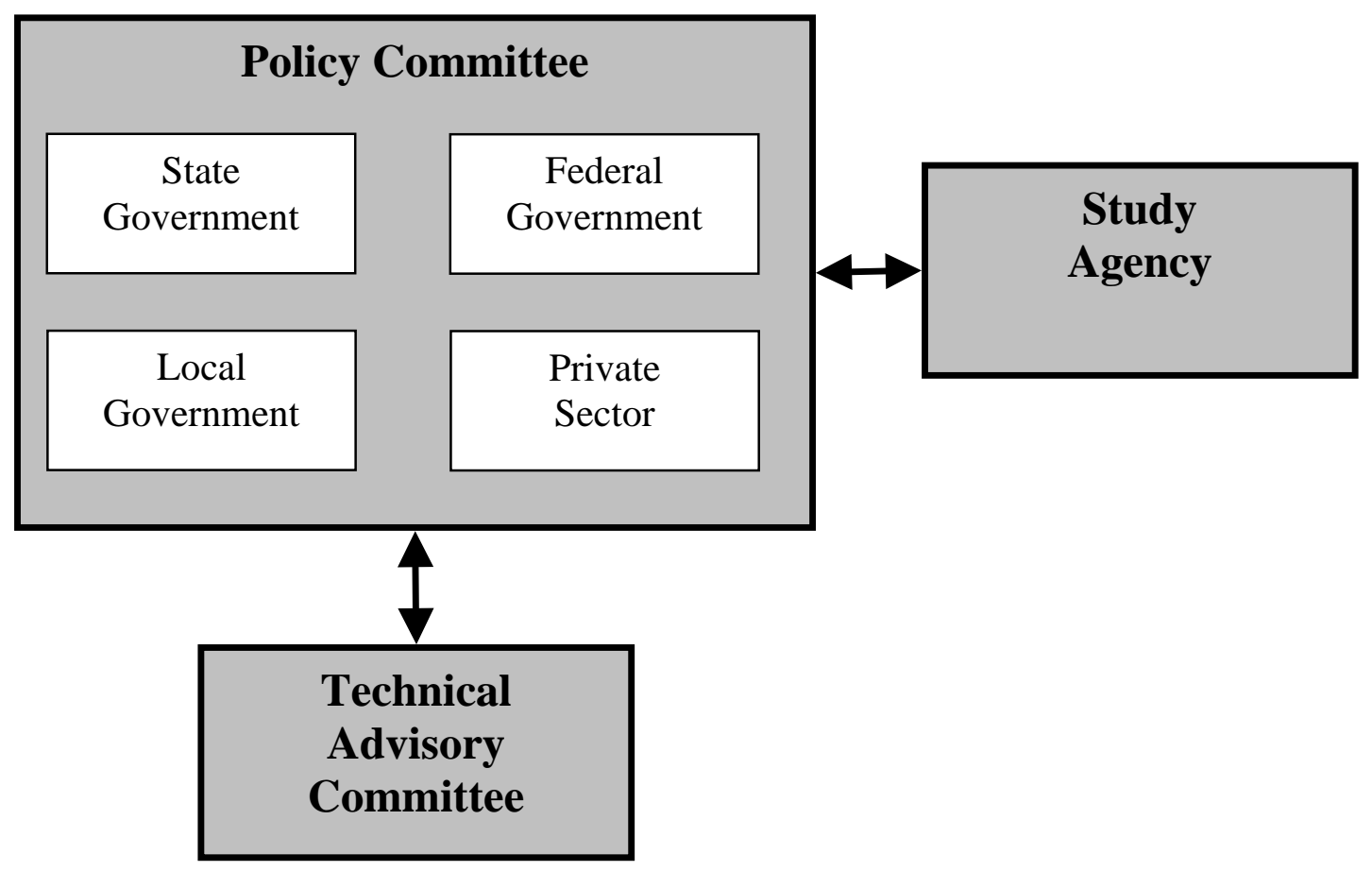

Three caveats suggest the SJVAQS and resulting SARMAP modeling were not purely consensus-based. First, not all stakeholders were involved in SJVAQS. Environmental coalitions were not represented (see Tables APPA-C and APPA-D). By comparison, the regulated industry helped fund the SJVAQS and had representatives on the Policy and Technical Committees.

Furthermore, as provided for in section 182(f) of the FCAA (1990), major point sources may request model simulations to determine if specific emissions controls are necessary to meet standards. In the SJV, the petroleum industry requested a simulation to determine if the SJVUAPCD's ozone plan would still meet the ozone standard if $\mathrm{NO}_{\mathrm{x}}$ controls on steam generators in the west side of Kern County were not implemented. The 
results of that simulation led to the removal of those controls from the plan. In this regard, SARMAP modeling had aspects of partisan and rational perspectives. It was partisan because not all emissions sources faced with possible control measures had an opportunity to question their necessity using simulation. It was rational, however, because modeling results were given authority as “truth” and provided justification for foregoing controls.

The second caveat is that there is a difference between the use of SARMAP to generate findings for the SJVAQS and to produce the 94 Ozone Plan. Although many of the SJVAQS committee members were involved in the execution of SARMAP for the 94 Ozone Plan, as well as commenting on the plan itself, there was an attempt to disconnect the two efforts. As the SJVAQS report stated,

Participants also agreed not to defer air quality planning and control activities until the completion of the study, thereby averting questions about the motivations of local and industrial groups participating in the study. (SJVAQS 1996)

The above quote speaks to the concern about conflicting interests, since some of the study’s funders might bear the costs of emissions controls. It also indicates that the planning and research efforts were disconnected, even if the impetus for SJVAQS was federal requirements to attain the ozone NAAQS by 1999. Regulatory deadlines, as well as a moral imperative to protect public health, necessitated a disconnect between research timelines and plan production.

The third caveat is that although SARMAP was the first major modeling effort for the entire SJV, its followed more than two decades of planning efforts in Central California. In the early 1980’s, the Western Oil and Gas Association (WOGA) sponsored a study of 
ozone forming processes in the Southern SJV (Blumenthal et al. 1985). The effort involved monitoring and application of the Urban Airshed Model (UAM), an early generation photochemical air quality model (Whitten et al. 1985). The results of that work informed plans for the SJVAQS in 1986. Also, considerable efforts had already been focused on Los Angeles and the Bay Area, involving many of the SJVAQS participants since the 1970's. Therefore, to understand SARMAP, the Policy and Technical Committees overseeing it, and planning efforts that led to the 94 Ozone Plan, it is necessary to explore the region's history of air quality modeling and planning.

\section{Getting into the Game}

In the late 1970’s, the Association of Bay Area Governments (ABAG) directed regional planning and, in turn, led efforts to develop the Bay Area’s Environmental Management Plan, which included a chapter demonstrating attainment of the ozone standard $^{76}$ (ABAG 1978). The director of that effort was Dr. Eugene Leong, who had several years' experience conducting technical studies for air quality planning in the South Coast Air Basin on behalf of EPA. He is now the Executive Director of ABAG.

Concurrently, a group at Lawrence Livermore National Laboratory (LLNL) was being sponsored by the National Science Foundation to develop the first regional-scale photochemical air quality simulation model, called LIRAQ (Livermore Regional Air Quality model). ABAG needed a system to associate emissions with air quality, as Leong said in 2002,

In 1972, when I was writing the first technical support documents for EPA, I was also moonlighting for SAI, Systems Applications Inc. as an analytical

\footnotetext{
${ }^{76}$ The original NAAQS for ozone was termed photochemical oxidant. The focus on ozone was made clear in the 1977 amendments to the FCAA.
} 
chemist. Phil Roth was developing the photochemical model there...I met Phil then, and was intrigued with his work...In my report,...I said these are the tools that should be used in the future for these plans.

LLNL needed an application for their new model. Their mutual needs and proximity made for a perfect match between the ABAG planning effort and the LLNL modeling.

Both ABAG and LLNL were relatively new to air quality planning; the incumbent was CARB. After observing EPA's bumpy entree into Los Angeles air quality politics earlier in the decade, CARB did not rush to join Leong's effort. EPA's grant empowered ABAG to form an interagency joint technical staff. CARB did not provide any technical assistance, but did send planners to participate along with representatives from BAAQMD, MTC, and Caltrans. In the words of Leong , ....in the early days of EPA and ARB interaction, ARB did not have much respect for EPA. It was from the standpoint that [EPA] are the new kids, they're environmental zealots, they don't know anything about reality. But, of course, EPA had the force of federal law.

EPA said, “Look, we don't have any choice.” ARB was saying, "We've been in this air quality business a lot longer than you. If you think that you can prepare a plan that meets those standards by those deadlines, go ahead and do it."

So, in the early days, ARB was not very helpful because they thought that the law was totally undoable. They thought that their own credibility would be lost by playing the game and saying we could meet these particular standards.

ABAG produced its oxidant attainment plan in 1978 as the product of the first application of an urban airshed model, LIRAQ. As Leong put it,

ARB realized that even they didn't have the capability to critique or judge our analysis. We would say, "if you don't like it, you do a better analysis." They were absolutely kept in bounds.

Following that whole episode in the late 1970's, ARB recognized that they better get into this game. If they don't get into the game, they're going to have consultants and local agencies basically bowl them over with analyses. 
That was the beginning of ARB saying, "We've got to develop this kind of in house capability.”

Modeling became the focus of planning, and one route to assert influence over emissions control decisions. Leong and others interviewed confirmed that public stakeholders were not involved in these early stages; state and local agencies, notably ABAG, controlled the modeling. This is not to say that public stakeholders were not at all involved in the process. They were, but their role was to submit written and oral comments on the drafted plan. ${ }^{77}$

Given that all interested parties, such as the regulated industry or environmental advocates, did not participate in the modeling that underwrote decisions, the process in the 1970's was more partisan and technocratic than pluralistic and consensual. Technicians had control of the evidence - LIRAQ modeling results - that was central to defending planning decisions. Challenges were rendered impotent without "better analysis. ${ }^{, 78}$ Furthermore, even though the technical evidence was highly uncertain, it was defensible. Of the plan, Leong said,

[W]e felt fairly confident that, within the bounds of certainty, this was a credible plan and it had a chance. Of course, none of us would have bet much money that it would, but we felt it was plausible. We used that word a lot. That this is a "plausible, technically defensible analysis."

The LIRAQ modeling that supported the Bay Area's 1978 Environmental Management Plan itself was understood by only a handful of engineers working at the cutting edge of new technology - computer simulation - on a relatively new planning problem - air quality management. With few, mostly technical personnel involved in the

\footnotetext{
${ }^{77}$ See Chapter II for a discussion of this decide-announce-defend approach to planning and the limits it places on meaningful public participation.

${ }^{78}$ See Leong quote above.
} 
modeling component of the planning effort, it was a technocratic process, whereby technocrats provided answers treated as the "truth” (Dutton and Kraemer 1985; Fischer 1990). However, the modelers believed only that the model-based plan was "plausible."

\section{Winning the Game}

Two decades later, by the early 1990’s, CARB had developed in-house modeling capability. They had a staff capable of evaluating others' modeling and conducting their own. For example, CARB did the modeling used to develop the 1994 ozone attainment plan in for the SJV. CARB was busy producing what Leong called "better analysis". Not only had CARB gotten into the game, they were winning it. Leong said CARB had technical capability for the "wrong reasons” so,

[ARB can] say, "We're going to do the modeling here at the state." But the modeling never sees the light of day because they don't like the answers.

There are, of course, many good reasons CARB might want to control modeling. As the state agency with responsibility to oversee air quality management, it is reasonable to expect CARB to want state-of-the-science tools. From a purely scientific perspective,

models are useful for studying complex atmospheric systems. Models codify and clarify the current state of knowledge, as well as lacking understanding, and can process huge quantities of data. Also, the courts expect agencies to make decision based on the best available scientific evidence, which is what models are presumed to produce. Such science is to provide the basis for decisions, otherwise decisions may be challenged as arbitrary and capricious. These reasons were justification enough for CARB to develop modeling capability. 
However, others familiar with the onset of modeling at CARB echo Leong's concern about the political motivations associated with modeling. Mr. Robin DeMandel, who has 30 years of experience conducting and overseeing modeling at the Bay Area Air Quality Management District, described CARB's use of modeling expertise,

From the first single layer model, the LIRAQ modeling in the 70's, all the way up to the present, the modeling systems have suggested that a VOCs strategy is what we need in the Bay Area.

ARB traditionally has been uncomfortable with that and has suggested that our modeling systems are not reliable enough. At the same time, they have suggested that the modeling systems used in other areas at other times have been. Sometimes it has been the modeling they've done, or the modeling done by others.

I'm suggesting there's a political bias here.

That "political bias" is toward controlling both $\mathrm{VOC}$ and $\mathrm{NO}_{\mathrm{x}}$ emissions, regardless of what the modeling indicates. Leong puts it,

[ARB] saw their job as reducing emissions, and reducing emissions blindly. Whether it is hydrocarbons, $\mathrm{NO}_{\mathrm{x}}$, whatever, we're just going to go after emissions. And they said there is too much uncertainty in the modeling, it takes too much work, so on and so forth.

Indeed, Dr. Alan Lloyd, CARB Chairman, went even further when he identified zero-emissions as a goal when he said,

And we have the chance to get closer and closer to zero emissions in every area (SBCAPCD 1999, page 3).

Also, CARB’s proposed 2002 Clean Air Plan says,

Finally, the Plan is intended to promote the cleaner, zero or near-zero emissions technology of the future for all sources, continuing the Board's international leadership role (CARB/CalEPA 2002, page ES-5).

A more measured position was indicated by Mr. James Sweet, SJVUAPCD, who said, 
Basically, we look at our standpoint from both responsibility perspectives. We're wearing the responsibility for the public, the responsibility hat for the government agencies that we belong to that we not do something that's burdensome and interfering beyond reason with the socioeconomic status of our counties. So that means you do as much as you need to do and no more. You keep that proper balance.

We want the model to tell us what the heck we're supposed to do. To accomplish our mission, which is to clean up the air without doing more than we need to do, without doing anything that is inappropriate.

Indeed, CARB has many rational reasons to uphold a policy of controlling both ozone

precursors, $\mathrm{NO}_{\mathrm{x}}$ and VOC (Landy et al. 1990; Bryner 1993):

- Burden of Proof - The current regulatory and legal structure favors preexisting activities. Laws and regulations were written long after the start of operations by major sources of emissions, such as oil refineries in the Bay Area. Chevron was already operating in Richmond at the turn of the century; the first major clean air legislation was adopted in 1970. CARB and air districts must justify emissions reductions strategies. ${ }^{79}$ Given the limits of our current knowledge and tools, such as models, generating conclusive evidence is often difficult (Jasanoff 1990; Jasanoff 1996a).

- Precaution - One approach to a precautionary principle is to suggest that emissions should be reduced unless they can be shown to be safe. (Of course, precaution can be used to create just the opposite argument: that costly controls should be avoided unless they can be shown to be beneficial and necessary.) The environmentalist precautionary position conflicts with the burden of proof challenge facing regulators. The FCAA supports agencies' approach to precaution (as opposed to regulated community's definition). For example, the law requires that health based standards for ozone, the ozone NAAQS, deviate conservatively from the threshold to allow "for an adequate margin of safety." (1970, Section 109(b)(1)) This margin, however, cannot be defined using scientific information; the EPA has relied instead on value judgments (Landy et al. 1990).

- Negotiation - Litigation is common; compromise is anticipated. It is therefore logical to take what may be viewed as an extreme position for the purposes of negotiation.

- So Far To Go For Ozone - In some places, such as the San Joaquin Valley, the state and federal ozone standards are violated so frequently, and estimated emissions are so high, that regulators may believe that there is very little

\footnotetext{
${ }^{79}$ The modeled "attainment demonstration" is one way to provide this justification.
} 
possibility of over controlling emissions. ${ }^{80}$ As discussed in later chapters, however, controlling emissions of both ozone precursors may exacerbate problems.

- Problem Definition: Ozone precursor emissions are known to contribute to other air quality problems, such as fine particulate matter formation, visibility, exposure to toxic organic compounds and aerosols, and environmental injustices. Studies currently underway in the SJV, as discussed in Chapter V: Central CA Air Quality, are intended to improve understanding of the role of emissions and appropriate responses to ozone, as well as other air quality concerns. Until those studies provide more definitive information, an interim strategy, given myriad air quality concerns and "so far to go" to meet ozone goals, a strategy of controlling both emissions may be deemed justifiable.

Given these myriad reasons, it is understandable that Ms. Lynn Terry, of the CARB

Executive Office, confirmed Leong’s claim, stating,

It is not a matter of using models to decide which is the most efficient control strategy. That has been the mindset for many of the industry supporters for SARMAP. I'm not sure that paradigm is going to ever be realized. It is not going to be a matter of choosing one strategy over another. It is a matter of whether or not it is doable.

From a certain standpoint we'd be better off never doing modeling again and just continuing to push the envelope on every strategy. Putting our money into new technology development and moving towards zeroemission technology for everyone.

But it is never that simple. Recognizing that we've had a process that, on the whole, has worked well from a public health standpoint, we've had huge reductions in air pollution in the State. It is fundamentally because we do the control strategies...The technical science behind that gives the typical policy-maker a little comfort so we're supportive of it.

...in a state where we have so far to go to meet health-based standards, there is no danger of too many reductions in the foreseeable future.

Another Executive Officer at CARB, Mr. Michael Scheible, put it this way,

Our agency's mission is to clean up the air. We've pretty much decided that reducing emissions is the way to do that.

Commenting on the use of SARMAP for the 94 Ozone Plan, Scheible said,

\footnotetext{
${ }^{80}$ See quotes by Terry and Scheible presented later in this chapter.
} 
Issues with the models that were telling us the role of $\mathrm{NO}_{\mathrm{x}}$ versus the role of hydrocarbon controls were more difficult to deal with. If [the model] does not have the science right, you could make some large mistakes. If the science is over- or under-estimating the emissions reductions by 10 percent, that's not terrible. By one calculation, it is a lot of money you do or don't spend. But in reality, I think, as we lower emissions we'll see air quality improve or not improve and we'll see that we're getting close. We can make adjustments later on, if need be.

The opinions of Leong and DeMandel suggest that CARB pursued a policy of “reducing emissions, and reducing emissions blindly." Statements by senior decisionmakers at CARB support the conclusion that CARB's default policy is to reduce emissions of both $\mathrm{NO}_{\mathrm{x}}$ and VOC emissions to the greatest extent possible. ${ }^{81}$

The statements do not, however, prove that modeling was used by CARB for partisan or technocratic purposes. The actual use of models remains hypothetical; evidence offered is anecdotal. To examine this hypothesis rigorously and systematically, two null hypotheses are:

- Null hypothesis \#1: CARB does not use models for partisan or technocratic purposes.

- Null hypothesis \#2: CARB uses models for consensual or rational purposes. Evaluative criteria are drawn from Dutton and Kraemer's (1985) definitions of technocratic, partisan, rational, and consensual processes to evaluate these hypotheses. To conclude that CARB does not use models for partisan purposes, decisions must be based upon modeling results. Models must derive the decision, rather than justify decisions founded upon other considerations, such as preconceived policy positions, political or economic concerns.

\footnotetext{
${ }^{81}$ See quotes of Terry and Scheible in the Narratives discussion later in this chapter.
} 
Whereas a partisan process uses models to support predetermined decisions, a technocratic one derives decisions based on the views of technical experts, as translated through the modeling. Modeling results are used in a technocratic process, but the results cannot be predetermined. For both partisan and technocratic processes, however, models provide the basis for emissions control decisions, and the modeling results are the product of objective, not subjective, analyses.

Respondents claim that decision-makers at CARB highlight modeling results selectively to support a predetermined policy of controlling emissions of both precursors. ${ }^{82}$ Or, that model results are continually revisited, using new metrics as necessary, to provide justification for policy positions. According to those who were part of the process, characteristics of the partisan and technocratic perspectives were present and significant. Modeling was a game that some partisans were not able to play, and modeling was essential for developing "defensible” plans. CARB did not rely on only the models to determine policy positions. Rather, the agency used models to support a predetermined policy of, in the words of Leong, "reducing emissions, and reducing emissions blindly.” Thus, CARB uses modeling for partisan purposes.

The veracity of this conclusion is undermined by the observation that, although no environmental coalitions were engaged in the SJVAQS, important elements of the consensual perspective were evident in the production of the 94 Ozone Plan. Decisionmaking for the SJVAQS was “cooperative”. The SARMAP modeling system was a key object of negotiation. Similarly, the development of modeling expertise and capability at CARB is indicative of a trend toward rational decision-making. 
The trouble here is a matter of degree. Models may serve many purposes simultaneously, and the uses may or may not be deliberate. As this case unfolds in more detail, it will become clear that other factors, notably time constraints and political will, overwhelm the modeling. This supports the finding that modeling is used to support decisions, rather than to derive them. Thus, it is possible to conclude that CARB's use of modeling is partisan. However, the conclusion is incomplete, since modeling was used in other ways too.

\section{Role of Modeling and Uncertainty - Consensus-based Planning}

This history of the development of modeling expertise at public agencies, such as ABAG and CARB, indicates the importance of modeling. Returning to the matrix offered by Dutton and Kraemer (1985), the evolution of modeling for air quality planning is mapped in Figure VI-3.

In the 1970's, modeling was a barrier to entry into the debate; technicians were in charge. To "get into the game" one had to be able to conduct or, at a minimum, critique modeling. CARB developed this capability in the 1980’s. By 1994, CARB had moved into a leadership position. It chaired both the Policy and Technical Committees and was executing SARMAP to support development of the San Joaquin Valley's 1994 Ozone Attainment Demonstration Plan.

${ }^{82}$ See quotes of Leong, Ziman, Wang, Sheible and Terry in this chapter. 
Figure VI-3: Transitions in the Political Perspectives on Models in Central California

\begin{tabular}{|l|l|l|}
\hline $\begin{array}{l}\text { Dominant Locus } \\
\text { of Control }\end{array}$ & \multicolumn{2}{|c|}{ Dominant Interest Served } \\
& Partisan/Self-interest & $\begin{array}{l}\text { Non-Partisan/ } \\
\text { Pluralistic Interests }\end{array}$ \\
\hline Technical Experts & $\begin{array}{c}\text { Technocratic Perspective } \\
\text { 1970's }\end{array}$ & $\begin{array}{l}\text { Rational Perspective } \\
\text { Model Uncertainty Info. }\end{array}$ \\
\hline Political Elites & $\begin{array}{c}\text { Partisan Perspective } \\
\text { Political Use of Models }\end{array}$ & Consensual Perspective \\
& & SJVAQS
\end{tabular}

What does this tell us about the role of modeling in Central California planning? Modeling expertise is a valuable capability that is like an ante to be paid to gain access to an important aspect of planning. It is a route for participating. Lacking expertise and constrained by the decide-announce-defend process, the lay public does not participate meaningfully. Yet, the regulated community remains in the game. It helped to fund the SJVAQS and had technical and policy experts serving on the Policy and Technical Committees. The process exhibits partisan, consensual and technocratic characteristics.

How would uncertainty information help or hinder the use of models by CARB? The answer is not obvious. The fundamental task of the rational approach - to consider uncertainty - is counter to the prevailing process. What potential is there for change? A shift towards a more rational decision-making process may threaten partisans. Partisans who exercise some dominance over the current process will resist a move towards the rational process unless it similarly suits their needs. Furthermore, the opinions of those with decision-making or policy-setting authority may change as personal viewpoints evolve through the acquisition of knowledge and expertise. Or, new people may ascend 
to positions of authority. These nuances will unfold as the description of the process deepens through the exploration of networks, narratives and decision-making constraints.

The observation that the regulators, not the regulated community, are the partisans controlling and benefiting from the modeling is surprising. As the narratives described later in this chapter demonstrate, however, CARB and air districts want to reduce emissions to pursue a host of air quality goals, not just the federal ozone standard. On the other hand, industry has good reason to pursue rational decision-making. It recognizes that uncertainty is costly and will gladly invest time and money to understand outcome probabilities. They want to make the best-educated decision possible and prefer to delay emissions control costs in hopes of better information. They face direct cost risks and would like to reduce risks using scientific research.

As this case study unfolds, divergent opinions and values are examined through the stories told by those interviewed. Scientific evidence and preferred policy responses provide two dimensions with which to distinguish these stories, referred to as policy narratives, and help us to understand how the models and management of uncertainties are incorporated into these narratives.

Prior to discussing the narratives, the networks involved in the production of the 94 Ozone Plan are presented and evaluated. Considering the political perspectives about the use of models per the theoretical construct of Dutton and Kraemer lacks resolution beyond the level of the organization. The next discussion delves into the interactions of the organizations and then goes two levels deeper; first, to the department or division level within key organizations, and, second, to the level of key individuals. This level of 
analysis indicates the nuances with which the different interest groups seek scientific information and, in turn, may use modeling and uncertainty opportunistically.

\section{The Actors}

\section{Networks}

Having examined the SJVAQS and production of the 94 Ozone Plan with respect to modeling control, it is informative to examine more explicitly the networks associated with the planning process. Networks describe the interactions between and within organizations. They are useful for tracking the flow of information and key intersection points. When considered in light of jurisdictions and authorities, important nodes are highlighted, either for the information they provide or the influence they exert.

In this case, the nodes of particular interest are CARB's Executive Office and Planning and Technical Support Division, the Planning Division at the SJVUAPCD, and stakeholders from the petroleum and agricultural industries. A network diagram for 94 Ozone Plan production is shown in Figure VI-4. It shows the many organizations involved and how they associated through a web of interaction. The thickness of the arrows indicates the degree of interaction, whereas the weights of the ovals around the SJVUAPCD and CARB indicate that these two organizations were integrally involved in plan production. The thick arrow connecting these two agencies indicates their extensive collaboration. In fact, the SJVUAPCD wrote and published the plan, per their statutory mandate, whereas CARB did the SARMAP modeling and assisted the air district with control rule development. ${ }^{83}$ The diagram shows the many routes of influence expressed

\footnotetext{
83 Some control rules did not originate at the SJVUAPCD because local districts have limited authority to exact emissions controls. CARB promulgates control rules for motor vehicles and interstate trucks; EPA
} 
by the regulated industries, most notably petroleum and agricultural companies

represented by industry associations.

The diagram indicates the lack of input that environmentalist stakeholders had in plan production. As in Tables APPA-D and APPA-E of Appendix A - Methods, none of the participants in the SJVAQS represented environmental organizations. Whereas environmental advocacy groups were active in the Bay Area, no such groups, nor recognizable individuals, were notable in the SJV during the 1990's. ${ }^{8485}$ Environmental organizations of interest to this research are what Yearly (1996) describes as those groups who "give voice to the idea that scientific evidence is manipulated by official agencies, and that government-commissioned scientists have asked the wrong sorts of questions" (Yearley 1996, page 183). Other types of environmental groups, what Yearly calls conservation organizations, such as the Nature Conservancy, were active in the SJV during the 1990’s pursing a land conservation agenda, but were not engaged in air quality planning directly.

Consulting organizations are not shown in the plan production networks, but they were present. For example, Alpine Geophysics mapped emissions estimates aggregated at the county level to the grid system of the SARMAP domain. Other consultants were involved too with plan development and the SJVAQS. Appendix E of the SJVAQS

has domain over motor vehicles in other states, as well as responsibility for cargo ships, airplanes, and trains.

${ }^{84}$ Recently, however, a environmental justice advocacy group was organized. The Central California Environmental Justice Network was formed to serve as a coalition to work on and get educated about issues of air, water and pesticide pollution (Anderson 2001).

${ }^{85}$ No claim is made here that environmental groups were deliberately excluded, simply that they were not involved possibly because they were not organized. In fact, environmental advocates interviewed for this research could not explain why environmental organizations were relatively silent regarding air quality planning in the SJV during the 1990's. 
summary of findings lists fifty-five contractors (SJVAQS 1996), categorized according to planning, field measurements, data analysis, emissions and modeling.

In addition to governmental organizations and consultants, some other groups deserve special mention. The Western States Petroleum Association (WSPA) helped to fund the SJVAQS and had active representatives on both the Policy and Technical Committees. WSPA represents a constituency of petroleum interests. Agricultural interests were present too. For example, the Nisei Farmers League was represented on the Policy Committee by its President, Manuel Cunha.

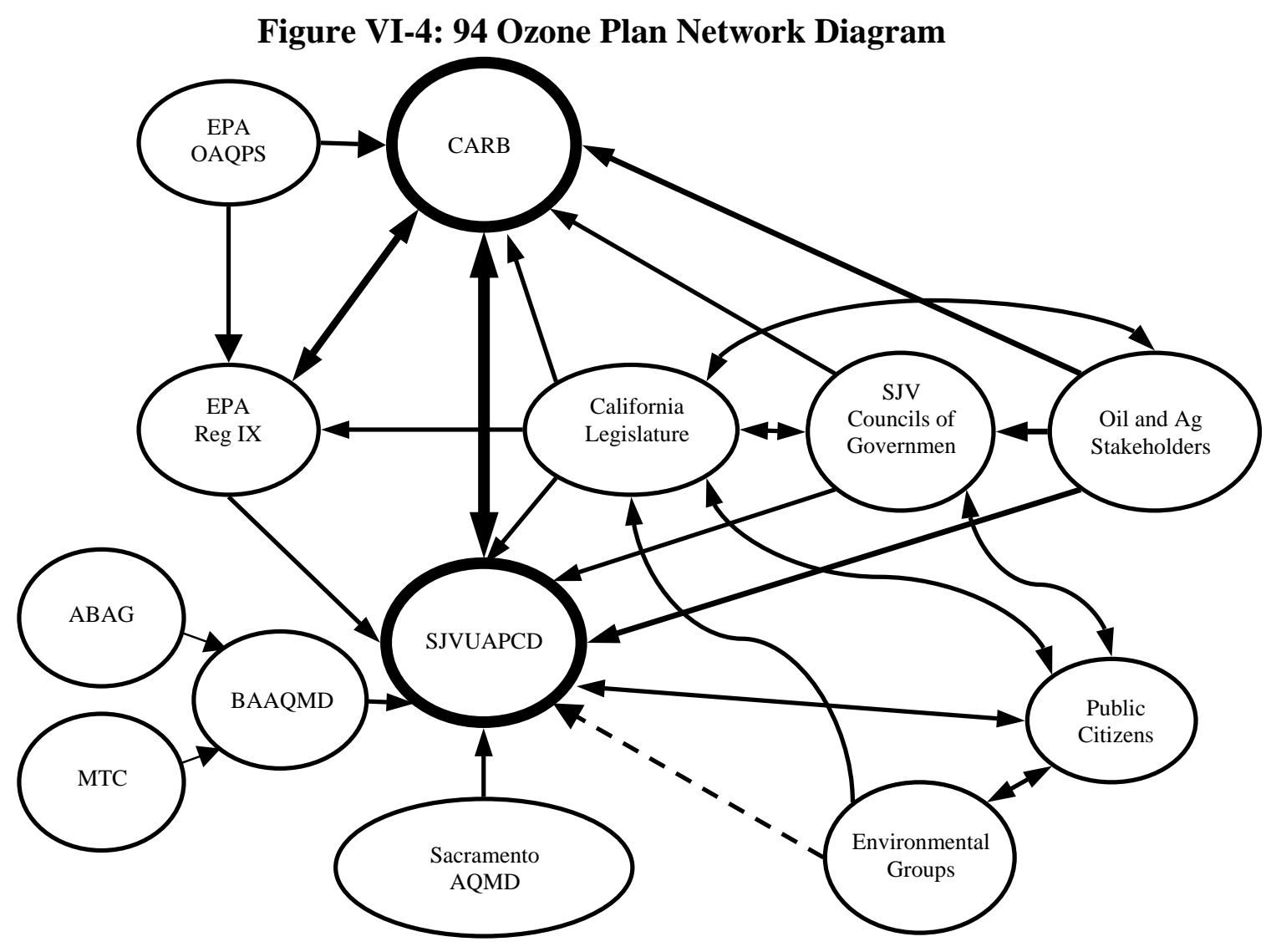


The diagram in Figure VI-4 does not show the difference between formal and informal interactions. Nor does it show what information is exchanged, authorities, or jurisdictions. Also relevant are the divisions within key organizations. There are divisions between the modelers and those who produce the emissions inventory at air districts and CARB. Emissions estimation and model execution require distinctly different activities. The emissions inventory plays three roles defined statutorily in air quality planning (FCAA 1990):

- Attribute control rules to source categories and individual “major” sources

- Provide data for Rate of Progress reporting, which is required by the federal CAA to show that air districts are reducing emissions at required rates

- Serve as inputs for modeling studies

Another division of note exists at CARB, where the executive policy-makers are in the Executive Office and the technical experts reside in the Planning and Technical Support Division. These divisions are shown in Figures VI-5 and VI-6.

Oftentimes, members of local government officials serve on the boards of planning agencies. The SJVUAPCD diagram reveals a link between county supervisors, city council members and agency board members, as demarcated with a thick gray arrow in Figure VI-6. This is an obvious and direct route of influence to which outsiders are not privy. For the purposes of this study, however, it is sufficient to acknowledge this linkage and its importance with respect to the production and use of modeling. As discussed in the next section, Information Exchange, modeling information is truncated and simplified long before it reaches the members of the board. 
Figure VI-5: Network of CARB Divisions for 94 Ozone Plan

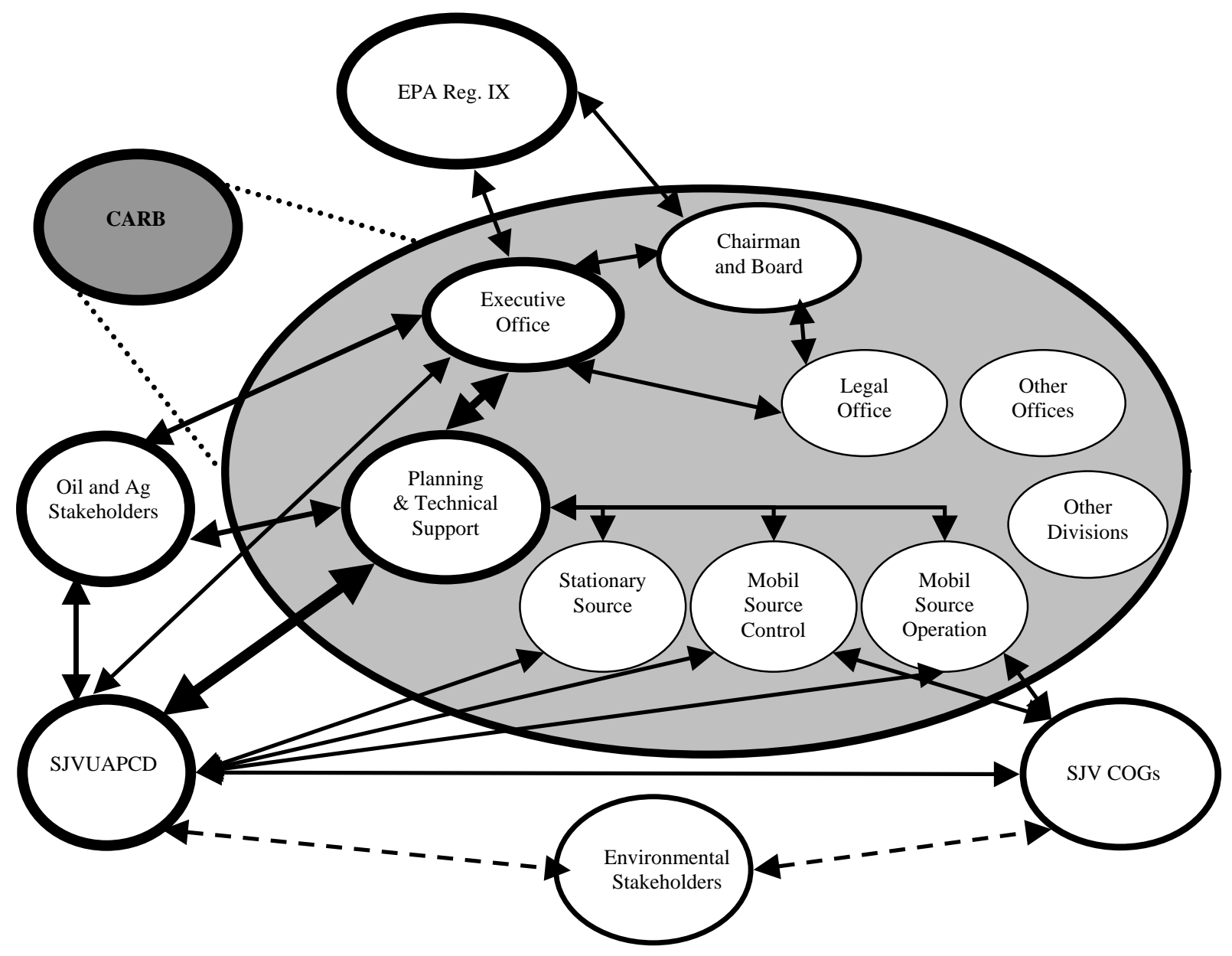


Figure VI-6: Network of SJVUAPCD for 94 Ozone Plan

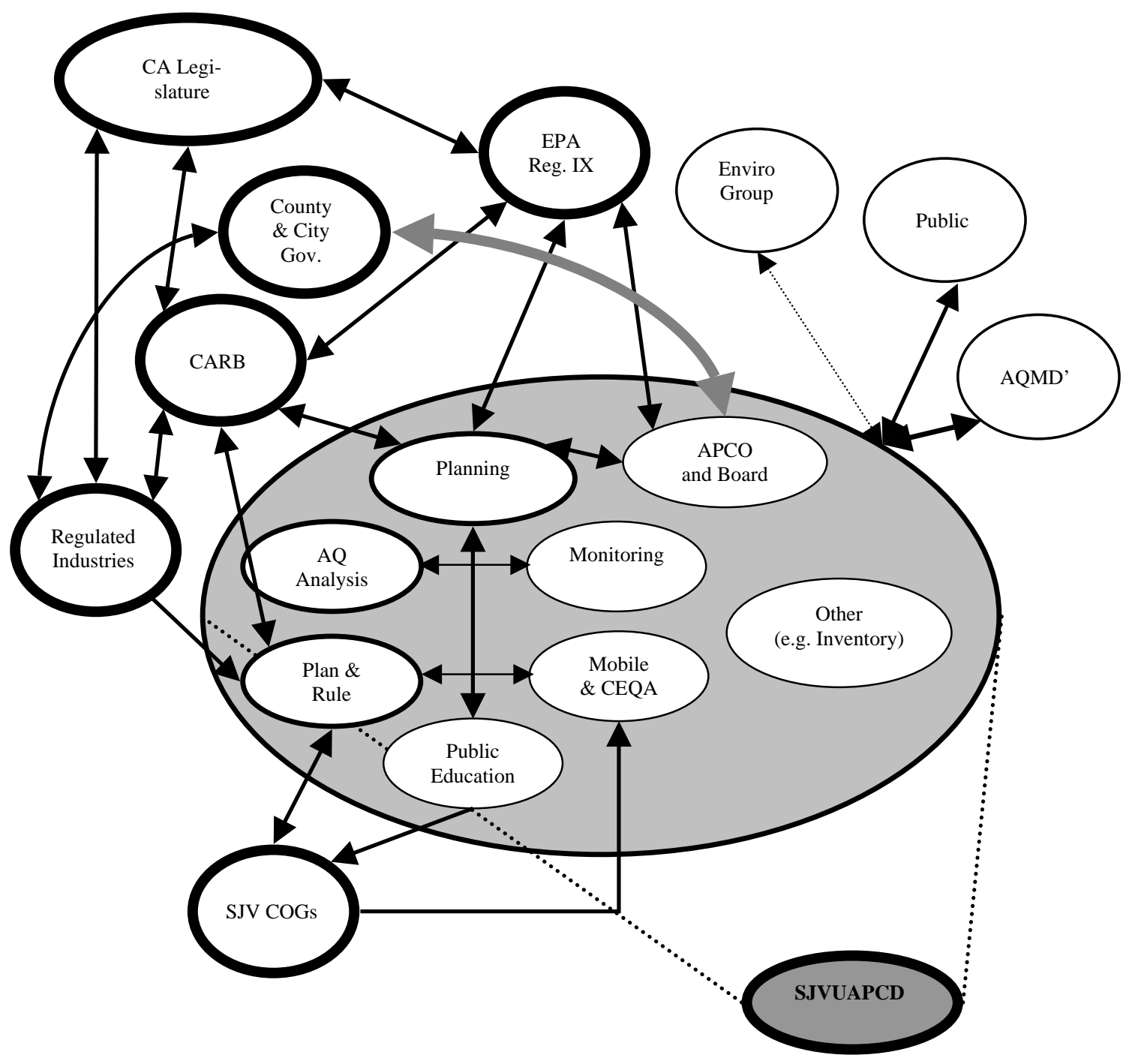


The SJVAQS Policy and Technical Committee memberships do not map directly to the networks already shown. Committee membership is listed in tabular format in the Methods chapter. Dr. Steven Ziman, Senior Staff Scientist at Chevron-Texaco Energy Research and Technology Company, described his role and the Technical Committee's charge,

The Technical Committee, for which I am one of the industry representatives, has been responsible for management of the design, development, and implementation of the studies. Field programs, data analysis, and so forth. All of these things which then become the basis for carrying out SIP development.

Our role ends nominally at the point at which decisions are made as to how a control strategy will be adopted for the SIP. At that point, my role changes to that of representing the industry in the formal and informal proceedings that lead to final adoption. We will comment on the strategies, the individual measures within the strategies, etc.

DaMassa described the difference between the two committees as,

[T]he Technical Committee was involved with review and decisions regarding all of the technical aspects of the study. The Policy Committee ....reviewed expenditures, progress, any decisions that needed to be made, the schedule, all of the policy level details. They controlled the budget.

Now that the organizations and divisions within organizations are mapped, a few disclaimers are in order. Neither the organizations nor their subdivisions are monoliths. There are differences of opinion within these organizations. Furthermore, individuals may differ considerably in the degree and veracity with which they espouse "official" policy or speak as freethinking individuals. For these reasons, interviews were conducted with more than one individual at key organizations (e.g., CARB, SJVUAPCD, BAAQMD). These individuals and their interviews are described in detail in the Methods chapter. 
The last disclaimer about the networks is that many of the key individuals have historical relationships that extend far beyond the 94 Ozone Plan network shown in Figure VI-4. Examples of these old relationships are provided earlier, such as Leong's work with Dr. Philip Roth at SAI in the 1970's, and are discussed more in Appendix A Methods.

Whereas the examples just provided highlight friendships, some interactions have been acrimonious and have led to longstanding disputes. Unquestionably, these formal and informal networks influence policy positions and opinions articulated regarding the SJVAQS and planning efforts in the SJV.

\section{Information Exchange}

The ability to define or "frame" the problem is an important source of influence. Whereas prevailing law, such as the Clean Air Act, defines the ozone planning challenge rather coarsely, those who, for example, select emissions controls or define hypothetical future scenarios for simulation are making important planning decisions. For example, one of the final scenarios simulated for the 94 Ozone Plan determined if refinery $\mathrm{NO}_{\mathrm{x}}$ controls on the Westside of the SJV were necessary to reach the ozone NAAQS. The choice of simulation reveals the degree of influence that project sponsors had in the planning process. ${ }^{86}$ Ziman said,

[SJVAQS participants] agreed that all sponsors of the study had the right to ask for modeling runs to be made to address certain issues. And we took that up in 1994 when the San Joaquin Valley had to decide whether or not to

\footnotetext{
${ }^{86}$ This is not to say that the simulation was unjustified given the magnitude of control costs, indications from prior research (see Blumenthal et al. 1985), and, in the long run, the models' indication that the controls were not necessary. Furthermore, the language of section 182(f) and EPA's interpretation of that language (see EPA 1992) required that major stationary sources use modeling to support any claims that $\mathrm{NO}_{\mathrm{x}}$ controls would be counterproductive or ineffective.
} 
implement $\mathrm{NO}_{\mathrm{x}}$ controls on sources on the Westside of the [San Joaquin] Valley.

Another way to exert control is through the sharing, or not, of vital information. For example, more than one respondent lamented lack of full disclosure about technical details, such as modeling assumptions. In fact, it is no longer possible to reproduce, exactly, the modeling conducted by CARB in support of the 94 Ozone Plan. Inventory files are long since lost, so too were two boxes of documents that were accidentally recycled from the CARB library. Lost or incompletely disclosed information is not necessarily the result of a conscious effort. As Sweet, an atmospheric modeler at the SJVUAPCD who served on both the Policy and Technical Committees, said,

It was a very open process. We did a lot of written memos that were not very formal in nature, but that was to try and help our documentation process. We have gone back to those to investigate certain questions since then. We found that despite the effort to document, there is never enough detail to really describe all of the aspects of the situation as to why you made the change in numbers. It is very difficult to do with the documentation.

Ziman, who has observed and participated in air quality modeling studies in California starting with the LIRAQ effort, notes that CARB did not provide documentation of their research in the 1970's and 1980's. Lack of disclosure made it impossible to know what went into the modeling studies. This is an example of how modeling may have obfuscated implicit value decisions embedded in agency studies.

Estimates of current and future emissions are a key input into simulation modeling. They also tend to be the greatest source of uncertainty in modeling results (Russell 1997; NARSTO 2000b). Because they are so uncertain, emissions inventories are undergoing constant update. Inability to track changes made to the inventory is one of the primary 
reasons it is not possible to reproduce past modeling efforts. Inventory files are simply lost or changed in undocumented ways.

The production of emissions inventories involves another network that, although contained within the network shown in Figure VI-4, is largely distinct from the modeling and the plan production networks. The organizations contributing information for emissions inventory development in the early 1990's were the SJVUAPCD, CARB, CalTrans, BAAQMD, ABAG, MTC, and Councils of Governments (COGs) in the SJV. Within these organizations, however, there are different subdivisions responsible for emissions inventory development.

The BAAQMD and SJVUAPCD produced emissions estimates for their areas of jurisdiction, in part using information provided by other agencies. ABAG, MTC and their counterparts in the SJV, COGs, provided baseline and projected estimates of vehicle miles traveled, demographics, and land-use projections. In so doing, they provided an essential, uncertain, and, for projections, impossible to verify modeling input. Often the projections are derived from other modeling efforts, such as vehicle emissions models. Government associations produced their information in the early 1990's with little oversight. As Terry described it,

I have concerns about their [COGs'] models being black boxes. Whereas for air quality models we have plenty of people who know the ins and outs and can run them independently, we just haven't had the resources to look at what they're doing and what they're giving us. Over time I'd like to do more of that and I've pushed on them to be more public in their process.

Air quality agencies' lack oversight of COGs. Emissions projections are developed by COGs by considering anticipated population and economic growth, and land use changes. Their lack of transparent methods for developing projections concerns others, 
too. Although respondents said the modeling does not incorporate political bias overtly, several noted that they were not including input data in their statement. Some claimed that county-level land use and VMTs projections were an opportunity for COGs to "game" the modeling. To be sure, lower projections of future emissions, or emissions producing activities, such as population or VMTs growth, make it easier to use modeling to show that standards will be met in the future. The extent to which there was an effort, successful or not, to use that opportunity is not easy to discover, though underestimation of VMTs is common. ${ }^{87}$

A separate process was undertaken to develop an "approved” inventory. Requiring approval from both CARB and EPA, as well as routes for public review and comment, the process becomes a constraint to updates, even if portions of the inventory are known to be missing or wrong. Sweet described the inventory development process as follows:

[R]emember that this was the first time we were gridding the emissions inventory. When you grid and start totaling it back up, we didn't have the UNIX capability to look at it graphically. So we had to get them [CARB] to make summary reports for us. Then we'd take a look at the categories and say, "Did they total back up to what was supposed to be put in?"

Sometimes they didn't. So we had to do a QA [Quality Assurance] effort on that to ask, "How come this category shows this much emissions when it should be some other number?"

[T] he way the emissions inventory worked at that time, all we could do is send up submittals to ARB. ARB put it into their system. They then had to send that information off to Alpine Geophysics to get the gridding done. They sent that back to ARB. We got ARB staff in the modeling section to do the summary reports for us so we could do QA. We then had to contact the emissions sections and Alpine to figure out why the numbers weren't what we expected, produce a recommendation for change, send it back to ARB. That was our process.

There's another area for error in what we were doing because we were short for time. We were doing, in single batch changes, corrections and day-

\footnotetext{
${ }^{87}$ Refer to Emissions discussion in Chapter IV.
} 
specific adjustments in the same iteration of file change. If you look back and see these emissions were changed in this amount. Well, why?

The process was inflexible to "last minute" changes. Discussing known uncertainties in the motor vehicle emissions inventory, Sweet commented,

At that point, we were using the best available information on emissions from motor vehicles. ARB was already well down the road for developing the running losses and evaporative losses that weren't in that model. So we knew that there was this huge chunk of emissions out there that wasn't going to be represented. But there was no way to deal with that.

There was discussion of how to deal with that during the modeling approach. I objected to some of the ways that people were planning to look at this.

One of the techniques that was used, they were looking at early morning emissions, trimming that as fresh emissions, comparing that to what was put in the model and say, "OK, here you have an underestimation."

And they were talking about possibly doing a construct where you would take that ratio and multiply your emissions to account for that error. I had significant problems with that approach. It completely dismisses the very concept of transport. It dismisses the carry over from previous day emissions. And it dismisses all other unquantified sources.

Furthermore, any change to the inventory, last minute or not, requires due process to allow for public review and comment. In 1994, changing the emissions estimate might have delayed modeling and, in turn, planning. Thus, it might have meant a tradeoff between improving the bases for decisions and submitting a plan within deadlines because the review and approval processes takes time.

In summary, control over the production and distribution of information is a means by which influence is exerted on the process. CARB and the SJVUAPCD are the primary producers and distributors of modeling information. However, these two agencies are dependent on others, notably COGs and the BAAQMD. Information production and review can be a constraint on the process too, as it must be approved by the public and oversight agencies. 


\section{Model Performance Evaluation}

"This stack, right here, is the most thorough model performance evaluation that I have ever seen,” said Mr. Don McNerny. He had reason to be proud. It was his staff that produced Performance Evaluation of SAQM in Central California and Attainment Demonstration for the August 3-6, 1990 Ozone Episode (CARB 1996b). Evaluation of the SARMAP modeling system was indeed the most extensive thus far for any such model applied in a regulatory context. McNerny said,

I'm pretty big on model performance evaluation. Every model should be challenged. In fact, I would like it if, when you pop up a model result, decision-makers would ask, "Why should I believe this modeling?"

When asked if decision-makers posed that question for the 94 Ozone Plan, he replied, No. They didn't. Hardly anyone asked that question. Most people don't want to know. They just want the result.

For those producing the plan, acceptable model performance is mostly a hurdle. The modeling must meet the requirements for performance set forth by CARB and EPA. Once it does so, it may be used for planning. ${ }^{88}$

After some initial performance evaluation, deadlines necessitated that the 94 Ozone Plan be produced prior to completion of more thorough testing of the SARMAP system. Eventually, however, CARB modelers conducted extensive tests based in part on suggestions made by the SJVAQS Technical Committee. Results from these latter tests were the basis for McNerny's report, but by then the SJV plan was already approved.

As McNerny continued,

My concern would be pretty much no one above my level would look at model performance. How is it doing? So I like to be able to sign off on it.

\footnotetext{
${ }^{88}$ For a description of CARB and EPA performance criteria, refer to discuss of Performance Evaluation and Validation in Chapter II.
} 
Others concur with McNerny. Terry, who is "above” McNerny in the CARB

Executive Office, explained how information is transmitted to CARB's Board,

As ARB staff, we tend to be pragmatic; to say, "Our basic framework is use the best science you have and meet the deadline." Those are the two fundamental goals. So when you come to the Board with a SIP, the Board is assured that, with these drop-dead dates we have to do the SIP, we've used the best information we have.

DeMandel concurred with Terry when he said,

As you go up the ladder to the Executive and Board level, you're more and more affected by and aware of the practical considerations, the legal considerations, the time constraints. Some of the political realities have to do with how the public is going to react to what we're putting out and how other agencies are going to react.

Down at my level, we're almost entirely focused on technical issues. We the technical people may say we don't have confidence in this analysis. The management may say, "Well, give us the best analysis that you have because we have to meet this requirement. We have to submit our plan on time.”

The results of performance evaluations are the most obvious indicators of uncertainties in modeling. Yet the information does not get communicated in any detail to planners and policy-makers. ${ }^{89}$

Instead of carefully weighing the results of model performance evaluation and then converting them into a conservative or alternative emissions reductions target, decisionmakers "internalize" the uncertainty associated with the modeling. Scheible, another in the CARB Executive Office, described how he deals with model uncertainty,

I think you mostly internalize it because you're not allowed by the process to manage it. The kind of questions I've found most interesting were: are we pretty sure if we do this amount of effort, it is not going to be severely

\footnotetext{
${ }^{89}$ Performance evaluation results are considered carefully by modelerst as they make improvements to the modeling system and proceed with the next round of modeling. The point here is that those who use modeling in the planning context are not so interested in how model performance translates to implications for control decisions.
} 
under control or severely over control? That this is going to put us in the right ballpark and this is going to get us close.

Similarly, Mr. Michael Wang, WSPA, relies on his experts to deal with the

uncertainty, but also trusts his intuition, as indicated by his statement,

I rely on [experts] to tell me whether or not they believe it. But I rely on a gut feel that asks if its seems intuitively correct. Air quality, in some respects, you can intuit whether or not directionally we're right, or it makes sense. Now there are a lot of counterintuitive aspects of air quality modeling, which is why sometimes we have difficulty making decisions that we ought to make.

But I think we are smart enough now to know where those counterintuitive situations may crop up. It's like playing golf, if you try and swing easier, generally it goes further. Well, it's counterintuitive, but we know that that's kind of the best way for most people to have a good swing. That's how I feel about air quality.

We know intuitively kind of the right way to do it. The counter intuitive examples are well enough known so that we can be aware of it when the time comes.

Terry displayed her view of the uncertainty, as well as the ongoing need for

identifying feasible emissions controls, when she said,

It always takes more to get there than we thought with the modeling in California. We have always been in a position of having to do every conceivable measure we can think of. We have never said, "We're not going to do that measure because we don't need it."

Mr. David Howekamp, Director of the Air Management Division at EPA Region IX from 1982 to 2000, said,

We knew that the public health standard violations were so bad in California that we would always err on the side of more emissions controls.

The effort committed to model performance analysis serves two purposes: one regulatory, the other research-oriented. Modelers do indeed learn from model performance evaluations, and they use that knowledge to improve performance. The regulatory use of performance evaluation is merely to assure oversight agencies, CARB 
and EPA, that the model meets performance criteria. Of the SARMAP application, Howekamp said,

We talked a lot about modeling performance. Targets were set early on for the modeling. We agreed on those targets. I think we put it in writing. In the case of the Valley that was how uncertainty was dealt with. It was in terms of performance targets.

The adequacy of these criteria for decision-making is discussed in the Chapter XIII SARMAP Modeling chapter. In short, modeling experts agree that these criteria are inadequate to assure reliable model performance. Here, it reveals that uncertainty information is truncated when transmitted to decision-makers. Rather than seeking detailed uncertainty information, decision-makers trust their technical staff to say "whether or not they believe it" and then they account for uncertainties in an informal, heuristic manner. In terms of regulatory compliance, performance targets simply need be met.

The influence associated with information production or dissemination can be further understood when juxtaposed with jurisdictional and decision-making authority. This task is taken up in the next section.

\section{Jurisdictions and Decision-making Authority}

Authority to control pollutant emissions, as well as to make plans, is split amongst federal, state and local agencies. No one agency has adequate authority and capability to withstand and supersede local planning politics, regulatory deadlines, and penalties for failing to meet them. The SJVUAPCD does not have dominion over all of the major emissions sources in its region, as shown in Figures VI-7 and VI-8, which are based on the inventories used in the 94 Ozone Plan. The SJVUAPCD must rely on EPA and 
CARB, and, to the extent that the Bay Area airshed is an "overwhelming" upwind source, the BAAQMD. The SJVUAPCD wrote that its "jurisdiction of control is generally limited to stationary sources while state and federally controlled mobile sources represent approximately 60 percent of the District's emission inventory” (SJVUAPCD 2001b). However, the jurisdictional boundaries are not always sharp. For example, air districts can enact transportation control measures to reduce VMTs and, in turn, mobile source emissions.

Figure VI-7: Emissions Jurisdiction of Control for 1990 VOC Emissions (Tons/Day) (SJVUAPCD 1994)

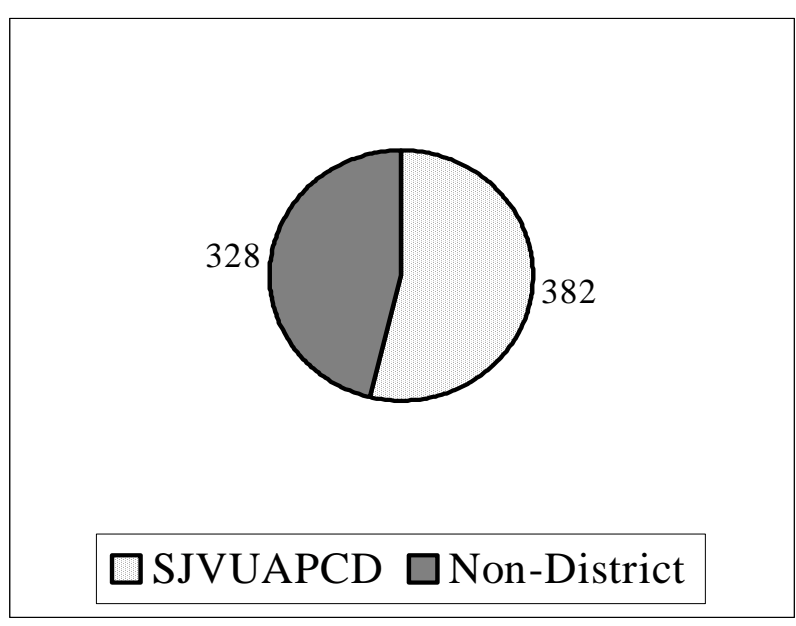


Figure VI-8: Emissions Jurisdiction of Control for 1990 NO $_{x}$ Emissions (Tons/Day) (SJVUAPCD 1994)

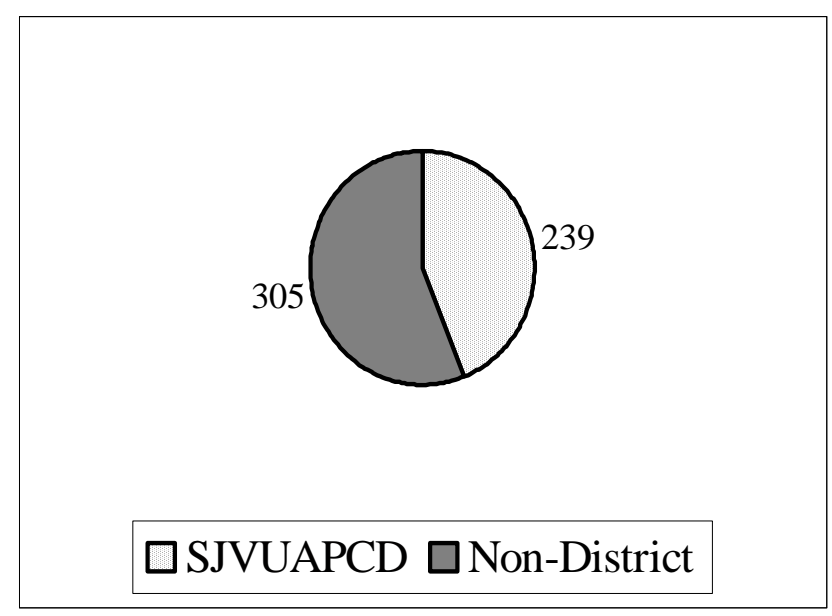

Another concern related to authority is the disjointed nature of air quality planning. The FCAA addresses pollutants individually. Mr. Victor Weisser, President of the California Council for Environmental and Economic Balance, spoke about not putting controls intended to reduce PM in an ozone plan, and also noted that some say the Bay Area plan should be for the Bay Area, not for the SJV too. Concerns about visibility, exposure to toxic air pollutants, and environmental justice provide impetus for emissions controls in the eyes of the public, but they are not necessarily easily incorporated into air quality plans focused on criteria air pollutants, such as ozone and PM.

As the federal agency charged with approving ozone plans, the EPA is the final arbiter of what constitutes an acceptable plan. EPA has disincentives to reject plans. Should EPA find a plan to be inadequate, it may approve only a portion of it, such as the planned controls, and then call for CARB and the air district to revisit the plan. If the plan's inadequacies are not addressed to EPA's satisfaction, EPA must produce a better plan called a Federal Implementation Plan (FIP) (FCAA 1990, Section 110). Where EPA deems the technical basis of a plan inadequate, such as modeling that does not 
"demonstrate" attainment, it would have to produce a better modeling study for its FIP. However, EPA recognizes that it may not be able to do any better with the modeling, especially where the inadequacy is due to a deficient observational data base.

Furthermore, the implementation of emissions controls based on an inadequate plan is seen as preferable to delaying control implementation in hopes of a better plan. For these reasons, one EPA official said EPA's decision-making authority is akin to the agency saying, "Do what we say or we'll shoot ourselves." Although EPA has the authority, it is loath to use it to reject a plan.

It is generally in the best interest of all parties to develop plans that can be and are approved. To facilitate approval, agencies interact throughout the planning process. By the time the plan is actually submitted to CARB and then EPA, it contains few surprises. Compromises have been reached, and EPA hopefully has little rationale to reject a plan. This leads to the conclusion that EPA has little decision-making opportunity in the ozone attainment planning process, instead choosing to exercise its authority informally and early in the process.

With respect to the production of the 94 Ozone Plan, the SJVUAPCD and CARB had the most decision-making power. Before being submitted to EPA, CARB must approve the ozone plans produced by air districts. In addition to leading the air quality studies in the SJV (i.e., SJVAQS, CRPAQS), interviews revealed that CARB had multiple roles developing the 1994 SJV ozone plan, including:

- providing key emissions estimates (e.g., motor vehicle emissions)

- managing the gridding of county-level emissions estimates 
- running the SARMAP modeling system

- identifying control rules that were "feasible" and "best available"

- setting statewide emissions standards for sources within their jurisdiction, most notably motor vehicles

- approving air districts' Ozone Attainment Demonstration Plans and compiling those plans into a SIP for submittal to EPA

The first three tasks related to emissions estimation and model execution fall within CARB's Planning and Technical Support Division. The Planning and Technical Support Division at CARB was engaged in the day-to-day planning efforts, working closely with the SJVUAPCD to complete the modeling. Identification of appropriate control measures and motor vehicle emissions standards is an iterative task that involves technical personnel, as well as executive-level input.

The role of the CARB Executive Office was to lead the SJVAQS Policy Committee, to oversee the work of the Planning and Technical Support Division, and to make recommendations to the CARB Board regarding the SJVUAPCD's ozone plan (i.e., to approve or not). In this respect, the Executive Officers were the executive policy-makers, with considerable authority to approve plans and to oversee information production. The Executive Office may have had a conflict of interest. One the one hand, it was responsible for assuring that technical evidence, such as modeling study results, was the "best available" and that decisions considered this information. On the other hand, the same office was responsible for overseeing the production of ozone attainment plans that they could endorse. This second responsibility may create a disincentive to bring into question the technical basis of the plan. Furthermore, it provides an opportunity to 
highlight modeling results selectively, or to interpret uncertain results to support predetermined decisions.

Role of Models and Uncertainty - Networks

What do these networks say about the uses of modeling in planning? Networks reveal the organizations and subdivisions that were important nodes in the network. Information producing, exchanging and approving responsibilities indicate influence when considered in relation to jurisdictions and authorities. Jurisdictions and authorities structure the interactions - who does what and why. Modeling was part key part of the process of information exchange and production. The costs of accepting responsibility for the production of model inputs and model execution were incurred for the benefit of exerting some control over modeling results.

In producing information, such as emissions inventories and modeling, and decisionmaking, such as approving plans, CARB had the greatest influence on the Central California air quality planning process. CARB's influence was located in its Executive Office, primarily, and its Planning and Technical Support Division, secondarily.

Others were influential too, notably the SJVUAPCD and COGs. The SJVUAPCD worked closely with CARB in producing emissions inventories and modeling scenarios. With help from CARB, the SJVUAPD also led efforts to disseminate information to the public. However, none of the groups were omnipotent. For example, neither CARB nor the SJVUAPCD had dominion over all of the important sources of emissions.

As the group doing the modeling, CARB's Planning and Technical Support Division might be said to control the production and dissemination of uncertainty information. 
Alternatively, the CARB Executive Office controls production of uncertainty information because it oversees the technical division and requests information from it. This argument has a snowball problem, though, since the Governor or the Legislature oversee CARB, though not on a day-to-day basis. Clearly, the most direct route of control rests with the modelers who are in the Technical and Support Division.

Responsibility for estimating emissions is given to the agency with dominion over the emissions source. The SJVUAPCD provides emissions estimates for major point sources, which is the same group included in their permitting program. The COGs oversee local land use conversion and public works. They provide projections of population changes and future land uses, including road construction and activity levels. There are, however, some disconnects. Although the COGs provide land use data, both CalTrans and DMV provide automobile data. Each of these bits of data contributes increments of uncertainty.

Although uncertainty information might be provided along with the input data, it is not. It would cost scarce resources to provide extra information with the input data. Notwithstanding the broader uses of uncertainty in planning ${ }^{90}$, such effort may be deemed unjustified if the recipient of that information has no plans to use it. In this respect, responsibility for uncertainty information resides with those doing the modeling. In this case, that responsibility rests with CARB, either the modelers or the policymakers. Consequently, uncertainty information is incomplete and it does not get presented in any useful manner to decision-makers. At best, uncertainty information is truncated when communicated, rarely is it interpreted except to say the model meets 
performance criteria. For example, the 94 Ozone Plan said of the SARMAP modeling application:

The model performance tests established by EPA determine if a model is able to successfully simulate an ozone episode that was observed. Since the model in its current form passes these tests, model improvements may effect the intensity of response to an emissions change but are not likely to alter conclusions to a significant degree (pages 2-10 to 2-11).

In this section, networks associated with the production of the 94 Ozone Plan are described and analyzed. Logical progression would now lead us to the level of the individual. In the next section, statements by individuals are considered in aggregate. The intent is not to understand individuals' objectives or motivations. Instead, the data are analyzed and then reconstituted and summarized as narratives.

\section{Narratives}

Stakeholder groups that agree share a common "narrative”. The narratives translate to recommended policy responses, so they are labeled accordingly. The policy outcomes pertain to the degree to which emissions of $\mathrm{NO}_{\mathrm{x}}$, VOC, or both are to be reduced in pursuit of air quality goals. Three narratives are evident in Central California air quality planning:

1. Control All Emissions: control all emissions of both $\mathrm{NO}_{\mathrm{x}}$ and VOC

2. Feasibility: control all emissions of both $\mathrm{NO}_{\mathrm{x}}$ and VOCs where it is economically and technically feasible to do so, unless there is certainty that the dual control strategy is wrong

3. Science/efficiency: use modeling and other scientific evidence to determine the most efficient control strategy, await the results of research before exacting costly emissions controls with uncertain benefits

The narratives are distinguishable by several topics:

\footnotetext{
${ }^{90}$ See Chapter III for discussion of the potential planning uses for uncertainty information.
} 
- Problem Definition - issues identified as central to the air quality problem, impetus for reducing emissions, veracity of evidence implicating the Bay Area as an important source of pollutant transport to the SJV, importance of $\mathrm{PM}$, visibility, toxics and environmental justice in addition to ozone

- Control Decision - justification for reducing emissions of both or one precursor; capability of models to justify control strategies that focus on $\mathrm{NO}_{\mathrm{x}}$ or VOCs reduction preferentially; relevance of debate about control strategy choice

- Modeling - current uses; potential utility; degree of precision and accuracy, capability to inform control strategy debate

- Performance Evaluation - purpose; opportunity to generate information about uncertainties in modeling; need to communicate performance results and to consider results in decisions

- Uncertainties - magnitudes; types; significance; uses of model performance evaluation results

The difference between the first and second narratives is the concept of feasibility. Whereas the first narrative does not accept prevailing definitions of feasibility, the second allows for economic or technical considerations when developing emissions control plans. Both the control all and feasibility narratives (referred to here as the two "control all” narratives) pursue a policy of reducing $\mathrm{NO}_{\mathrm{x}}$ and VOC emissions simultaneously to address a host of air quality and social goals, including unhealthy levels of ozone.

The science/efficiency narrative places emphasis on control plans that await conclusive evidence before implementing emissions controls. This narrative is concerned with economic efficiency and speaks of the social cost of emission controls. Comparatively, the control all narrative is focused on the social costs associated with incompletely known health impacts of emissions. 
Like the science/efficiency narrative, the two control all narratives discuss the science. All of the narratives forward arguments using scientific evidence. Yet, none of the narratives forward a purely scientific perspective because they operate in a dialogue that is at the interface of science and politics. What distinguishes the narratives is how values and politics overlay onto available scientific evidence and use uncertainties to construct their positions.

The control all narrative without feasibility constraints is a zero-emissions value position. The narrative does not trust regulators to define feasibility because it assumes industrial and economic interests exert influence to effectively bias the decisions emanating from the agencies. This view concurs with Yearly's characterization of environmental advocacy groups' "distrust of parts of the scientific establishment and of the authorities' use of science” because they "are dependent on scientific knowledge produced by other persons or agencies, knowledge which is suited to the objectives and agenda of those other groups” (Yearley 1996, page 183). At the same time, however, these organizations do believe that honest science will reveal fundamental truths about the dangers and damages of polluting activities.

Environmental interests did not present themselves explicitly in this case, so the control all narrative was more like a compass point. It established a position against which the other narratives located. Ironically, the science/efficiency narrative views the regulators as providing proxy for environmental interests. Indeed, the control all narrative provides the backbone of the feasibility narrative. It also contributed to the political mood at the time in the form of lawsuits, both threatened and filed, and the resultant need to assure that control decisions were defensible in court. In short, the 
feasibility narrative is the control all narrative tempered by pragmatic and political realities, including a willingness to accept agencies' definitions of feasible.

\section{Problem Definition}

The feasibility and science/efficiency narratives diverge at the outset with the definition of the problem. Whereas the former is concerned with a host of air quality goals, including ozone, particulate matter, visibility, and toxics exposure, the latter focuses on efficient strategies to pursue standards. Ziman expressed these divergent views when he said,

I think that's a philosophy that's always been at ARB: We need both hydrocarbon and $\mathrm{NO}_{\mathrm{x}}$ reductions, and not only are they going to be good here but they're going to be good for PM.

Eventually that's true. It may be the worst of all worlds. You're basically forcing reductions on both sides that are much more significant than trying to find the best pathway.

From a position in the CARB Executive Office, Terry affirmed Ziman’s observation and demonstrated a broader problem definition than ozone and PM concerns. In so doing, she also retorted the science/efficiency narrative, when she said,

When you design your strategy to meet the ozone standard, you have to take into account your other public health needs. So you say, "I'm not going to attain ozone and make it harder to achieve particulate matter standards. Or forego toxics benefits, particularly from a localized standpoint. Community health and environmental justice are the other overlay to this debate. Putting the blinders on and saying we're going to focus all of our effort on this one public health issue just doesn't fly anymore.

Scheible, Terry's colleague in CARB's Executive Office, added a similar comment,

Well, you have an ozone model. But you also have PM concerns. You have a lot of other air quality concerns you're dealing with. Those get weighed against a model and a Federal requirement that, for this spot, you attain the ozone standard by this date. A scenario like that would be one of many of us saying, "Well, the model may show a disbenefit from $\mathrm{NO}_{\mathrm{x}}$ controls in a certain area, and those $\mathrm{NO}_{\mathrm{x}}$ controls in the model may make it harder to get 
to that point for ozone, but we have other compelling reasons why we need $\mathrm{NO}_{\mathrm{x}}$ reductions. They aren't as precisely quantified.”

Scheible added,

Basically, I think the industry view was, because I think they thought it would come out to the answer they wanted, and maybe they believed the science, that the model would say there's a different way to do this than requiring Bay Area $\mathrm{NO}_{\mathrm{x}}$ refinery controls.

We said that's one element of it, but we have to look overall at the role of what $\mathrm{NO}_{\mathrm{x}}$ does and whether or not from a total pollution standpoint, whether or not cost-effective $\mathrm{NO}_{\mathrm{x}}$ requirements make a lot of sense. We viewed that they did.

Howekamp indicated that EPA shared this view when he said,

I didn’t buy the technical evidence from the Bay Area locals [that $\mathrm{NO}_{\mathrm{x}}$ controls were counterproductive]. There were downwind, long-range impacts, plus $\mathrm{PM}_{10}$ and $\mathrm{PM}_{2.5}$.

In later chapters, the scientific justification for implementing $\mathrm{NO}_{\mathrm{x}}$ controls in response to "other compelling reasons" that are not "as precisely quantified” is discussed at length. Here the important point is that some believe other concerns justify a control all strategy, even if the science had not provided definitive proof of the relationships.

\section{Control Decision}

Later in the interview, Terry described how both the business and public health communities are not “comfortable” with a policy of controlling all emissions feasible, and how they tend to align with the science/efficiency narrative,

To be honest, the business community is never going to be comfortable just saying, "Let's do everything possible to reduce air pollution as quickly as we can." Nor is the public health community going to be satisfied with that. Everybody wants to feel like we have a defined target and that it is fundamentally a scientific endeavor to understand air pollution and to reduce it.

The science/efficiency narrative views a dual-precursor control strategy (i.e., control all) as ignoring available scientific evidence suggesting it is the least efficient option. It 
associates the feasibility narrative with regulating agencies (i.e., air districts, CARB) and laments that the regulators fail to abide their fiduciary responsibility to meet air quality goals while minimizing social cost. Understandably, the regulated community espouses the science/efficiency narrative. Many technical specialists similarly further this view.

The California Council for Economic and Environmental Balance (CEEB) represents the science/efficiency narrative with its air quality program mission statement,

To advocate policies that provide for necessary improvement in air quality and at the same time support California's business climate. To ensure that air quality requirements not only improve air quality, but are also cost effective, equitable and workable from an operations standpoint. (CEEB Website: http://www.cceeb.org/documents/aq_foldover.html)

This quote belies a focus on cost-effectiveness decision criteria while considering both economic viability and environmental quality. Both of these themes are key features of the science/efficiency narrative.

Respondents residing in the Planning and Technical Support Division at CARB demonstrated a split between narratives. On the one hand, they have a predisposition to rely on the results of the scientific evidence that they help to produce. On the other hand, it is unreasonable to expect any significant deviation from executive level policy - the feasibility narrative.

\section{Models and Uncertainty}

A third dimension by which narratives are distinguished pertains to the uses and usefulness of models and the uncertainty information models may be used to produce. The science/efficiency narrative relies on available modeling studies, as well as other research, to define an asymmetrical shape of the ozone production possibility curve. 
Given the asymmetry, this narrative would like to find the most efficient (i.e., shortest) distance to the ozone standard.

The feasibility narrative considers the production possibility curve to be unknown for different regions of Central California and time periods of an ozone season other than the one or few simulated ozone events. Consequently, the narrative conservatively assumes the curves to be symmetrically convex. Preconceived notions obviate modeling and other technical evidence. A simple mental map wins out over complex and uncertain modeling results.

The science/efficiency narrative is summed up by Wang, WSPA,

If one believes that the ROG/ $\mathrm{NO}_{\mathrm{x}}$ ratio, and the EKMA diagrams...if we believe what that says, there is a relationship that we should pay attention to. By not paying attention to it and by doing everything we can, we may be ignoring some very fundamental truths.

Ziman clarified Wang's concern when he said,

We started off in the late 1980's doing VOC [controls] only. Then we started doing $\mathrm{NO}_{\mathrm{x}}$ [controls]. Now we do $\mathrm{NO}_{\mathrm{x}}$ and VOC...If you look at the mobile source, that is a $\mathrm{NO}_{\mathrm{x}}$ and VOCs strategy. You start looking at that for the Bay Area and we're going to run right down the isopleth.

In referring to the isopleth, Ziman is considering modeling that defines the ozone production possibility curve in the form of an ozone isopleth, as well as other corroborating evidence, such as ambient measurements. As explained in the next chapter, the isopleth is akin to a production possibility function. For ozone, it is understood to be nonlinear. However, the narratives disagree about how well we can trust the models to define the shape of the curve. 
Those who hold the feasibility narrative viewpoint deem isopleths unreliable. Instead the models are useful for only general indication of direction and to provide justification for moving forward with emissions controls.

The science/efficiency narrative acknowledges the limits of the isopleth construct, but refers to it, along with other corroborative research, as the best available evidence. The narrative accepts that there is such a place as a ridgeline where ozone production is most efficient, and that a control strategy of reducing both precursors will reduce ozone concentrations less efficiently than a single-precursor strategy. Instead, argues the science/efficiency narrative, regulators should call for controls that move the regime toward a region of least efficient ozone production, a point off of the ridgeline. Furthermore, failure to do so necessitates additional controls to meet ozone standards. This last point is central. It derives from the nonlinear relationship between precursor emissions and ozone concentrations. This issue is discussed with examples in the next chapter.

\section{Model Performance Evaluation}

Model performance evaluation is another attribute of the process that distinguishes the narratives. The evaluation offers the chance to characterize how well the model is simulating phenomena of interest. According to the science/efficiency narrative, it is often a missed opportunity. Furthermore, the timing of the evaluation, responses by the planners and technicians to results, and eventually, the communication of results, provide further clues about the use of the modeling in plan development. 
When planners understand that the modeling is uncertain, but know their plan calls for "every conceivable measure we can think of","1 , they conclude that more model uncertainty information has limited utility. If the constraint is available controls, in the eyes of executive decision-makers, it also renders useless the debate about controlling $\mathrm{NO}_{\mathrm{x}}$ or VOC emissions preferentially. The science/efficiency narrative, however, says this perspective is too simple. Given the nonlinear relationships, the dual control strategy might make necessary more controls of both precursors, thereby exacerbating the problem of finding “feasible” controls.

In the preceding discussion, key arguments associated with the three narratives attending Central California air quality planning are presented. The quotes presented are a very small percentage of the data, both written and oral, considered in developing these narratives. In Table VI-A, a more extensive comparison of the two dominant narratives feasibility and science/efficiency - is presented to extend and summarize their description.

${ }^{91}$ See quote of Terry in Narratives discussion earlier in chapter. 


\section{Table VI-A: Comparison of Feasibility and Science/Efficiency Narratives}

\begin{tabular}{|c|c|c|}
\hline Attribute & Feasibility Narrative & Science/efficiency Narrative \\
\hline $\begin{array}{l}\text { Problem } \\
\text { Definition }\end{array}$ & $\begin{array}{l}\text { - Ozone, PM, fine PM, visibility, } \\
\text { toxic VOCs } \\
\text { - Interdependence of pollutant } \\
\text { problems } \\
\text { - Environmental justice } \\
\text { - Growth and vehicle miles traveled } \\
\text { - Short planning deadlines } \\
\text { - Prescriptive laws and regulations } \\
\text { - Inadequate public support for } \\
\text { controls }\end{array}$ & $\begin{array}{l}\text { - Ozone } \\
\text { - Inadequate science or understanding } \\
\text { - Politically-driven decision-makers at } \\
\text { agencies } \\
\text { - Inadequate resources for research or } \\
\text { control implementation } \\
\text { - Trade off between clean air and } \\
\text { economic vitality }\end{array}$ \\
\hline $\begin{array}{l}\text { Planning } \\
\text { Constraints }\end{array}$ & $\begin{array}{l}\text { - Available "feasible" controls } \\
\text { - Regulatory deadlines } \\
\text { - Requirements for open planning } \\
\text { processes and public participation } \\
\text { - Public understanding } \\
\text { - Public willingness to reduce } \\
\text { emissions }\end{array}$ & $\begin{array}{l}\text { - Scientific understanding } \\
\text { - Uncertainty assessment and } \\
\text { management } \\
\text { - Open-minded policy-makers } \\
\text { - Regulatory deadlines } \\
\text { - Dual control strategy may have made } \\
\text { the situation worse }\end{array}$ \\
\hline $\begin{array}{l}\text { Efficacy of a dual } \\
\text { control strategy }\end{array}$ & $\begin{array}{l}\text { - Ozone, PM, visibility, toxics } \\
\text { exposure reduction are all reasons to } \\
\text { control all emissions feasible } \\
\text { - With "so far to go" both precursors } \\
\text { must be controlled to extent feasible }\end{array}$ & $\begin{array}{l}\text { - Unknown benefits in the SJV } \\
\text { - Worst option in the Bay Area }\end{array}$ \\
\hline $\begin{array}{l}\text { Utility of } \\
\text { NOx/VOCs } \\
\text { debate }\end{array}$ & - Not a productive debate & $\begin{array}{l}\text { Fundamental to agencies' fiduciary } \\
\text { responsibility }\end{array}$ \\
\hline $\begin{array}{l}\text { Role of Models in } \\
\text { Plans }\end{array}$ & $\begin{array}{l}\text { - Provide directional indication and } \\
\text { identify reductions targets } \\
\text { - Satisfy regulatory requirement for } \\
\text { an attainment demonstration } \\
\text { - Enables planners to say they used } \\
\text { the "best tool" } \\
\text { - Improve understanding }\end{array}$ & $\begin{array}{l}\text { - Used to support a priori policy } \\
\text { positions } \\
\text { - Should be used for uncertainty } \\
\text { information and to prescribe the most } \\
\text { efficient control strategy } \\
\text { - Improve understanding }\end{array}$ \\
\hline $\begin{array}{l}\text { Model accuracy/ } \\
\text { precision }\end{array}$ & $\begin{array}{l}\text { - Too uncertain to identify a VOCs or } \\
\mathrm{NO}_{\mathrm{x}} \text { strategy, but can use model to } \\
\text { understand problem for directional } \\
\text { guidance }\end{array}$ & $\begin{array}{l}\text { - Uncertain, but methods exist to } \\
\text { evaluate control strategies }\end{array}$ \\
\hline $\begin{array}{l}\text { Model } \\
\text { Uncertainty }\end{array}$ & $\begin{array}{l}\text { - Uncertainty is not used now in plans } \\
\text { - Uncertainties are internalized by } \\
\text { decision-makers, and model } \\
\text { uncertainty is just one part of it }\end{array}$ & $\begin{array}{l}\text { Essential to develop plans that } \\
\text { consider uncertainty, but any } \\
\text { contingency controls must be based on } \\
\text { the science }\end{array}$ \\
\hline $\begin{array}{l}\text { Utility of } \\
\text { Uncertainty } \\
\text { Information }\end{array}$ & $\begin{array}{l}\text { - Not used explicitly now, so there is } \\
\text { no place for it }\end{array}$ & $\begin{array}{l}\text { - Only way to develop efficient and fair } \\
\text { plans }\end{array}$ \\
\hline $\begin{array}{l}\text { Method to } \\
\text { Manage } \\
\text { Uncertainties }\end{array}$ & $\begin{array}{l}\text { - Internalize it in decisions } \\
\text { - Continue to invest in the science to } \\
\text { reduce important sources of } \\
\text { uncertainty }\end{array}$ & $\begin{array}{l}\text { Thorough assessment of model } \\
\text { uncertainties that goes beyond } \\
\text { regulatory requirements for } \\
\text { performance evaluation } \\
\text { - Decision-making that considers } \\
\text { uncertainty explicitly and rigorously }\end{array}$ \\
\hline
\end{tabular}


The translation of these narratives to the decision space is shown in Figure VI-9. The science/efficiency narrative wants to use available evidence to determine the most efficient control strategy, thereby implying a $\mathrm{NO}_{\mathrm{x}}$ or VOC-focused strategy. The feasibility narrative defines needed controls as some function of political will, and then translates it to the decision space. It is thus operating along a different dimension, political will, that is eventually mapped to the constrained decision space with dimensions of $\mathrm{NO}_{\mathrm{x}}$ and VOC controls. The control all narrative, strictly defined, does not allow for feasibility criteria.

Figure VI-9: Narratives Mapped to Control Decision Space

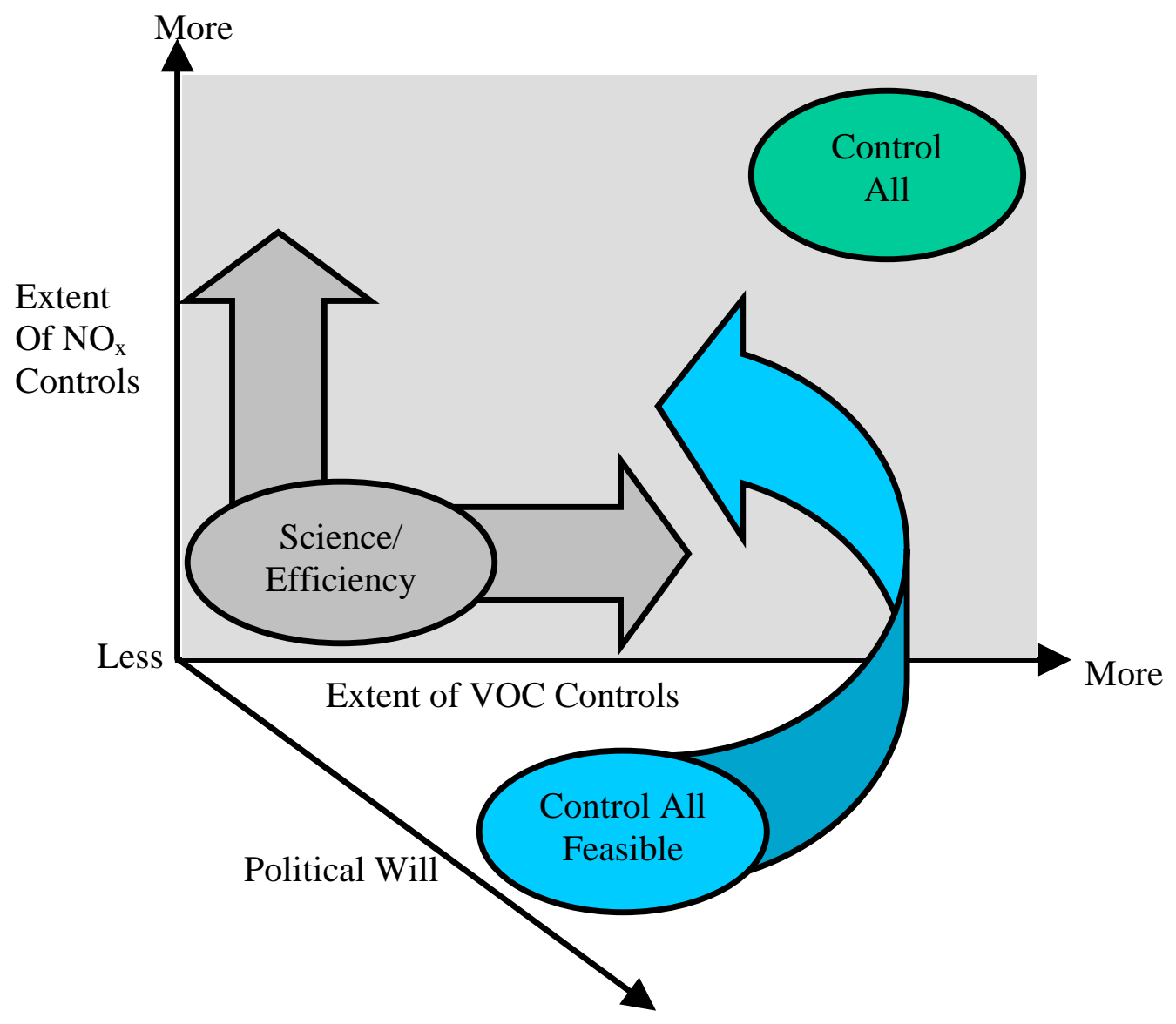


The importance of any one narrative depends on the influence of those who espouse them. Understanding the narratives, and when one dominates, is essential for understanding the role of models and the potential for additional modeling information, such as uncertainty, to be incorporated into decision-making. In this case, the feasibility narrative dominates because it is located in the Executive Office at CARB, air districts, and at EPA Region IX. Whereas some respondents claim that CARB policy coincides with the control all narrative, and there is some evidence of that narrative emanating from CARB (for examples, see CARB/CalEPA 2002, page ES-5; SBCAPCD 1999, page 3), the agency is legally required to evaluate the technical and economic feasibility, as well as cost effectiveness, of controls before requiring they be implemented.

Whereas Figure VI-9 shows the narratives mapped to the decision space, Figure VI10 presents a matrix that distinguishes the narratives according to the degree of focus on science or politics.

\section{Figure VI-10: Matrix of Narratives}

\begin{tabular}{|l|l|c|c|}
\cline { 3 - 4 } \multicolumn{2}{c|}{} & \multicolumn{2}{c|}{ Emphasis on Science } \\
\cline { 3 - 4 } $\begin{array}{l}\text { Degree of } \\
\text { Politics }\end{array}$ & Less & Feasibility & More \\
\cline { 2 - 4 } & More & Control All & Science \\
\hline
\end{tabular}

The above matrix warrants several points of clarification. First, it indicates a science narrative that is not identified in the case. A scientific narrative might be best described 
as objective and disinterested. It provides backbone to both the feasibility and science/efficiency narratives.

A second point about the matrix is that it is too simple. More dimensions are necessary to isolate the narratives, such as stories about problem definition, interpretations of the regulatory structure, and preferred responses to uncertainty, are essential for delineating the narratives. These additional data make it possible to distinguish beyond the control all and science narratives.

The third point of clarification is the extremes of the axes in the matrix. Less science is a long distance from no science. All of the narratives refer to and rely upon the science. Similarly, no narrative is devoid of politics.

\section{Shared Opinions}

The narratives highlight and organize the areas of disagreement. Of course, there are many shared opinions and observations about air quality planning in Central California. Whereas narratives diverge with the definition of the problem, agreements pertain to the constraints on the planning process, the characterization of uncertainties in planning documents, and the actual uses of the modeling.

Respondents tend to agree that the air quality planning process faces several constraints. Although none of the respondents provided a succinct, organized summary of these constraints, their statements generally fall into four categories: (1) mandated planning tasks, (2) timelines and deadlines, (3) political will, and (4) requirements to based decisions on the best available science. Although these terms might be associated 
with constraints on any planning process, their manifestations in this context warrant explanation.

\section{Mandated Planning Tasks}

Legal and regulatory requirements establish the impetus for planning (e.g., air quality standards), planning tasks, and deadlines for meeting standards and submitting plans. Oftentimes, the deadlines set in the law are long passed, so judicial rulings establish the new deadlines. For example, the deadline for submitting an approved 1994 Ozone Attainment Demonstration Plan for the SJV was the result of a court settlement (CARB 1994, page I-10). Planning agencies would like more time to conduct the plan development process, including the search for emissions reduction options.

As second aspect of the law constraining planning is the need to assure that decisions are neither arbitrary nor capricious in the view of the court. Respondents spoke often of the litigious nature of the planning process. Actions and decisions typically anticipate subsequent lawsuits. Therefore, the other constraints - time, political will, and science and legal requirements are interdependent.

\section{Timelines and Deadlines}

Timelines for planning never match research schedules. At some point available technical information, notably the modeling system and emissions inventory, must be taken as "best available" for use in the plan. Ziman described how the process of getting comfortable with the SARMAP modeling system was truncated by planning deadlines,

We had [SARMAP] developed for [the SJVAQS] and we were comfortable to the extent that we could be. But, we were also uncomfortable because in the midst of trying to do a reasonable performance evaluation we were forced by the agency to drop everything and just do the SIP because of the 
time frame. And, so we never finished the evaluation to the degree it should have been done.

As Nester said,

[W]e're under a sanctioned timeline to produce a plan that includes modeling. In essence, we don't have the luxury of time, we don't have unlimited resources. We're going to go with what we've got when it comes down to it.

Time represents a different kind of constraint for the environmental and regulated communities, as both want more time to critique and negotiate plans through written and oral comments. Furthermore, industry would rather planning deadlines slow down to allow the science to inform decisions. They do not want to implement costly controls until the science has confirmed that the controls will yield desired results. There is also a financial incentive to delay incurring emissions control costs. That incentive is even greater when the effectiveness of controls is uncertain.

\section{Political Will}

A less obvious constraint is political will, but it is omnipresent in the minds of decision-makers. As DeMandel described it,

I think that people down here in the trenches feel that we're doing everything we can. We also have to meet our legal requirements, State and Federal. We have to turn in plans on time. They have to follow whatever the law says. But as a practical matter, I think they feel, "What more can we do?”

If we could think of more control measures that are practical, that you could actually do, then we would propose them. By that I mean you have to consider whether something costs so much that it wouldn't fly. Or that it produces such a great social upheaval that it wouldn't fly. We could have odd-even driving and probably reduce emissions a lot. But it's not going to fly. You try this, and they'll criticize your agency because you're a pest!

So you have to think, "Well, what's practical? What could we actually do that would work and that would be politically acceptable?" 
More than one person interviewed for this research suggested that political will may exist to further ratchet downward allowable emissions from smokestack industries, but not to exact controls on activities directly influencing the general public. Ziman described how political will translates into control decisions and obviates modeling results,

Even if you were to look at it and say, “The modeling says I'm going to get very, very little air quality improvement by putting additional controls on this industry. But I may actually get something by doing no-drive days. God forbid. Or I might get something by controls that will hit the consumer directly.”

He will see the pass through very directly. Otherwise it's a hidden cost. There's no politician in his right mind who is going to pass that.

We'll [petroleum or power industries] get hit and those others won't. There is not a politician, in my way of thinking, who could withstand the pressure of essentially making a decision that is not as much political as technical.

It is not legally defensible to omit an emissions control option because it is deemed politically unacceptable. Instead, the concept manifests formally via the definition of what is feasible. Assessment of the SJV indicates that difficult socioeconomic problems (e.g., high unemployment, low incomes) may undermine efforts to implement costly controls in pursuit of improved air quality. Air quality may, quite simply, be a luxury that the SJV cannot afford. Petroleum and agricultural employment may conflict, at least rhetorically, with the need to reduce pollutant emissions from these sectors. These concerns relate directly to air quality planners' lament that sufficient “feasible” emissions are not available. The BAAQMD defines feasible as:

Feasible control measures are those measures which are: (1) reasonable and necessary for the San Francisco Bay Area; (2) capable of being implemented in a successful manner within a reasonable period of time, taking into account economic, environmental, legal, social, and technological factors; 
and (3) approved or approvable by the California Air Resources Board, based upon State law and ARB policies (BAAQMD 1997).

This definition is too flexible to obviously reject or accept any proposed control measure, since words like “reasonable” accommodate anything deemed politically acceptable. In practice, other factors, such as politics and the timelines necessary for rule-making, influence the selection of control measures. The BAAQMD definition of feasible also defers, albeit slightly, to CARB.

Air quality controls have focused historically on large smokestacks and automobiles. Industrial operators feel like scapegoats. Indeed, as discussed in ChapterIV: Central CA Air Quality the petroleum industry was responsible for over 40 percent of the emissions reductions achieved in the SJVUAPCD between 1990 and 2001, with controls on mobile sources comprising most of the rest of the reductions (CARB 2002). In some regions of the SJV, such as Kern County, emissions associated with the petroleum industry are still significant (see discussion of emissions in the Central California Air Quality chapter.) However, meeting the ozone and particulate matter standards in the SJV will require controls on non-point sources, such as agricultural operations, trucks and passenger autos. The latest emissions inventories show a greater contribution from non-point sources associated with mobile and agriculture sources.

As noted in the previous section, concern about political will distinguished the two control all narratives. Political will concerns lead to a form of incrementalism that does not attempt to push control plans much beyond the status quo (Greenberger et al. 1976). Regulators do not want control plans to be perceived as Draconian. ${ }^{92}$ The regulated

\footnotetext{
${ }^{92}$ Draconian is a term referring to an unreasonably harsh system of punishment implemented by an Athenian leader, Draco, in the $7^{\text {th }}$ Century.
} 
community uses the term to good effect. For example, in a letter to Sam Armentrout, Mayor of the City of Madera and Chairman of the SJVUAPCD Board, an industry coalition noted that failure to attain the ozone NAAQS "will result in draconian penalties for all Valley residents and businesses” (Reheis-Boyd 2002).

Shipp used the term in a similar way when he said,

In the modeling portion of it, again the politics fall in the uncertainty. For instance, we've been at this process for a long time. I have some experience outside of the [San Joaquin] Valley. I have an idea of the way the SIP planning goes, because I've been through three or four SIPs. We didn't make attainment in any one of those SIPs. So my feeling is to go conservative.

Others' feeling is that you can get into the Draconian measure if you have to go conservative. If the [reduction goal] is 50 percent, the types of things you'd have to do wouldn't be palatable. Let's say no [agricultural] burning in the Valley. Let's say no drive days. All of those things don't go over so well with the public.

Greenberger et al. (1976, page 39) note that incremental decision-making "limits the burdens that are imposed on [the decision maker's] knowledge and comprehension and minimizes the uncertainties that he must reckon with”. Consequently, the need for policy analysis (e.g., modeling studies) is reduced when its primary purpose is to reduce uncertainties. Policy-making through incrementalism "serves the purpose of policy research” (Greenberger et al., 1976, page 40). Incrementalism is substituted for and used to hedge against uncertain science. Mahlman (1998, page 104) similarly characterized the process leading to the Kyoto Protocol, an international agreement to reduce climate warming emissions, as "quietly and wisely" providing "some point that allows incremental action” in the face of uncertain models. 
In this case, incrementalism is also a safe policy approach that obviates modeling. It relates to political will through the definition of feasible. The definition of feasibility constrains the set of available emissions controls so that additional controls called for in successive rounds of planning are "incremental” rather than being perceived as Draconian.

\section{Decisions Based on the Best Available Science}

Planners cannot, at least officially, cast aside models. The FCAA requires they be used unless another suitable approach is identified. The fourth constraint acknowledged generally by respondents is indeed the science. Most generally, lack of scientific certainty constrains decisions. Such certainty is neither forthcoming nor anticipated, so it does not really influence day-to-day planning decisions. More relevant in this case, science is a constraint due to its special status in the regulatory and judicial realms.

Regulatory guidelines define the tools to be used, such as modeling systems, and insist that science considered in decision-making be the "best available". Similarly, the courts trust when regulators claim their decisions are based on the best available science that the decisions are neither arbitrary nor capricious.

The models need to confirm that planned controls will bring the district into attainment. Before being used for this “attainment demonstration”, they must meet lax standards for performance set forth by CARB and EPA. These same two agencies review and approve plans, and they must determine the plan itself to be "plausible." Here, though, plausible is not defined explicitly, like the another pivotal word, feasibility. 
Instead, plausibility is some function of technical defensibility and the judgment of personnel at EPA and CARB.

Given myriad constraints, respondents agreed that modeling results do not drive planning decisions. Other constraints are more important. The planning challenge is to conduct a modeling demonstration that, with "best available" information and within timelines, supports emissions controls plans constrained by political will. Per federal law, modeling is used to show that plans will yield air quality goals. Mr. David Jones, Planning Manager at the SJVUAPCD, recalled the modeling attainment demonstration as follows,

Since we already had come up with a list of measures, and we were basically trying to do the biggest ones, we threw them all into the modeling. Initially, the modeling didn't show attainment. When we were contemplating what else we could do, the decision was made to change some of the boundary conditions and some of the wind patterns. When they did that, all of the sudden we were down below $120 \mathrm{ppb}$ in Bakersfield and $123 \mathrm{ppb}$ in Fresno, or something like that.

The above statement reveals that available politically-tolerable controls is the constraint, and that modeling is conducted until the set of controls yields a simulation “demonstrating” attainment. The rhetoric associated with the modeling, however, indicates that control plans build from the modeling, when just the opposite is true. Respondents acknowledge this fallacy. Respondents similarly agree that the 94 Ozone Plan tells two stories about the science and uncertainty. The plan's Preamble says,

The Attainment Demonstration Plan uses a computer model to simulate the future air quality in the Valley while reflecting the effects of measures proposed to curb pollution. The model is complex, new, state of the art, and undergoing continuous refinement. Nevertheless, it is expected that the model will serve as the preeminent tool for local, state, and federal agencies, the public, and industry, to evaluating current and proposed air quality 
planning efforts. The model has predicted a District-wide attainment of the federal ozone standard by 1999.

The District is certain that over the life of this Plan that new information may be forthcoming which could impact and require modification of the Plan. Such modifications to the Plan could include: (1) updates and corrections to the District's emission inventory, (2) updates and corrections to the Ozone Model, and/or (3) elimination, additions, or substitutions of control measures based upon new technical or economic data. (SJVUAPCD 1994, page ii).

Despite these disclaimers, the plan proceeds with the attainment demonstration using an emissions inventory suggesting a level of precision and accuracy that was not justified given the known, and acknowledged, limits of available information. Little mention of modeling or emissions inventory accuracy is made, except that the motor vehicle emissions inventory is “known to be underestimated” (SJVUAPCD 1994; page 5-8). Emissions are reported to the nearest ton (per day) although the estimate may be only accurate to the nearest 100 tons. In acknowledgement of the nonlinear chemistry, the plan concludes that it needs either a 47 percent reduction in domain-wide $\mathrm{NO}_{\mathrm{x}}$ emissions or combined 40 percent VOCs and 38 percent $\mathrm{NO}_{\mathrm{x}}$ reductions to meet the federal ozone standard. This portion of the plan did not account for the uncertainties, such as the representativeness of the modeled episode or known biases in the model.

Respondents concur that the plan is written in this way to meet the requirements of the law. McNerny said,

Yeah, I think that is the process. It's move forward to meet this exact target. And there is a lot of uncertainty in the target and control measures and the prediction of what the emissions will be ten years out that hasn't been considered.

Terry agreed, but added that she was not so concerned about the model's uncertainty, 
We have a legal requirement to pick a number. We pick the best number we have with the information we have. But we also know that the fundamental value of the tools is directional.

I know that there is a discomfort level with saying, "We acknowledge that the model is only directional but we use it to establish a specific target."

But that's the law and it has been an effective driver for new clean air strategies.

As these statements indicate, even though uncertainty was known to be a concern, the legal construct did not allow for any more than its rhetorical acknowledgement. The requirement to "pick a number" does not allow for any uncertainty. The legal context is black and white. The model either demonstrates attainment or it does not. There is no gray area.

\section{Summary of Chapter IX: Description}

This chapter describes the air quality planning as it occurred in Central California during the 1990’s. It starts with a broad, theoretically-based assessment of the process by examining who controls the modeling and whether partisan or pluralistic perspectives are represented. A second level of analysis is network diagrams. The final level delineates narratives based upon statements of individuals familiar with the process. Narratives summarize and organize the different stories attending Central California air quality planning. Having defined opinions in juxtaposition, through narrative analysis, the chapter closes with identification of shared opinions about what constrains air quality planning decisions. 


\section{CENTRAL CALIFORNIA PLANNING PART II - DEBATES AND DECISIONS}

\section{A. Introduction}

This chapter summarizes the impetus for air quality planning, and describes the options facing decision-makers by presenting a decision heuristic: an ozone isopleth. The isopleth is introduced in economic terms as an ozone production possibility curve.

Debates about the air quality problem are surveyed, and then actual decision outcomes are noted and discussed.

\section{B. Three Key Planning Requirements}

\section{Ozone Attainment Demonstration Plans}

Under the FCAA, local air districts must produce Ozone Attainment Demonstration Plans (Ozone Plans) when they violate National Ambient Air Quality Standards for any one of the six air pollutants. Only ozone and particulate matter concentrations violate the NAAQS in the SJV or the Bay Area (see Fed. Reg. 1993, 1999a, 2001).

The plans of particular interest in this case pertain to ozone. Ozone precursors $\left(\mathrm{NO}_{\mathrm{x}}\right.$ and VOCs) also contribute other air pollution concerns, notably particulate matter (PM) . This research focuses on the 1994 San Joaquin Valley Ozone Attainment Demonstration Plan (94 Ozone Plan), but also considers ozone plan produced by the Bay Area in 1999 and 2001, the 1997 SJV PM $_{10}$ Attainment Plan, and interim progress reports.

\section{Clean Air Plans}

The California Clean Air Act of 1988 established state air quality goals for ambient concentrations of the criteria pollutants. Clean Air Plans (CAPs) are required every three years when observations reveal violations. Models need not be used to support these 
plans, nor are there planning or attainment deadlines established in the CCAA. Instead, “reasonably practicable” progress must be made toward meeting the state standard. Precursor emissions must be reduced by five percent annually, or, if five percent reduction is deemed infeasible, then "all feasible measures" are to be implemented in an “expeditious” manner (CCAA, Section 40914 (b)(2)).

\section{Transport Assessment and Mitigation}

In addition to CAPs, the CCAA requires CARB to evaluate the transport of pollutants between air districts. When transport relationships are found, emissions controls are required to that are "commensurate with the level of contribution” (CCAA, 2001).

\section{Decision Spaces}

The question facing planners developing a plan to meet the federal ozone NAAQS is: How much must current $\mathrm{NO}_{x}$ and VOC emissions be reduced to meet the ozone NAAQS (by a specified deadline)? The decision space is shown later in Figure VII-1. Borrowing a concept from economics, the figure shows a production isoquant, which is defined as all the possible combinations of two inputs that are physically capable of producing a given rate of output (Miller, 1982). ${ }^{93}$ A production possibility curve depicts the maximum amount of output to be produced from two inputs. In this case, the output is ozone, and the inputs are ozone precursors, $\mathrm{NO}_{\mathrm{x}}$ and VOCs.

Although it includes the relevant decision space, Figure VII-1 does not frame the problem as viewed by planners. Economists are concerned with producing more product per unit of input, whereas air quality planners want less product from less inputs. That is,

${ }^{93}$ Although the definition is concerned with a constant rate of production, ozone production rates need only 
they want to produce ozone in the least efficient manner possible. They need to choose how many tons of emissions to reduce, if any. A more useful depiction of the ozone production possibility curve is Figure VII-2, which essentially flips Figure VII-1, by changing the axes from precursor emissions to emissions reductions.

With the control decision properly framed in Figure VII-2, it is possible to get to the heart of the problem. What matters is the shape of the isoquant. It is not likely to be the smooth curve shown in the figures due to the complex, nonlinear chemistry involved. For the production of a simple product - one that relates linearly to inputs - it is intuitively and theoretically consistent to consider the curve convex, especially approaching the axes.

The law of diminishing marginal returns indicates that, holding all other variables constant, as equal increments of an input are added, beyond a certain point, the resulting rate of increase in product will decrease (Miller, 1982). The law applies to ozone formation too. As more VOCs are emitted into the atmosphere, at some point the availability of $\mathrm{NO}_{\mathrm{x}}$ will limit ozone formation. At and beyond this point, the addition of VOCs will not increase ozone unless more $\mathrm{NO}_{\mathrm{x}}$ is added too.

Although the general shape of the curve can be intuited at the extreme values of emissions inputs, it is not possible to predict the shape elsewhere in the decision space. Such intuition is confounded by the nonlinear relationship between the precursor pollutants and their product, ozone, as well as significant determinants of ozone not depicted in the diagram. Factors influencing ozone but not represented include local

be sustained for 1-hour or 8-hours, which are the averaging time for ambient ozone concentration standards. 
geography and meteorology, other pollutants found in a polluted atmosphere, and spatial and temporal variability. Usually, the isopleth axes include only anthropogenic emissions, even though biogenic emissions may be significant contributors to ozone formation, because emissions decisions pertain to anthropogenic, not biogenic, sources.

As drawn, the isoquants imply continuity. Control decisions, however, involve incremental reductions. For example, installation of selective catalytic $\mathrm{NO}_{\mathrm{x}}$ reduction on major sources will reduce emissions by a large increment.

Without any understanding of the shape of the ozone production possibility curve, it is reasonable to endeavor to reduce emissions of both ozone precursors to assure progress toward the ozone goal. It is the job of the simulation model to define the shape of the ozone production possibility curves at varying levels of emissions.

Isoquants are not new to ozone planning. One of the first generations of photochemical air quality simulation models capable of depicting non-linear chemistry, the Empirical Kinetic Modeling Approach, produced an ozone isopleth. The isopleth is the visual depiction of modeling results, including the sensitivity of peak ozone to changes in precursor emissions. It typically presents the relationship as an ozone production isoquant (i.e., Figure VII-1), rather than an ozone reduction isoquant (i.e., Figure VII-2).

The creation of isopleths is a key output of the modeling. Without models, it would not be possible to describe the shape of the ozone production possibility curve. The surface in the isopleth is generated using a modeling simulation that has been able to reproduce an observed ozone episode with an acceptable level of accuracy. The model is 
exercised many times, each with a different assumption about the amount of precursor emissions. Each modeling run provides a point; many points eventually create a surface depicting the sensitivity of peak ozone to changes in precursor emissions in one meteorological situation. Using one ozone episode, and associated meteorology and emissions estimates, it is then possible to map ozone sensitivity to changes in emissions within areas of interest, including urban centers, locations of peak ozone, and locations downwind from major sources of emissions.

Throughout this analysis, a fundamental question is the role that models play in the planning process. One way to address the question is to identify where information produced by the models, such as isopleths, is incorporated into decisions, if at all. Furthermore, the characterization and communication of the isopleth's uncertainties is an indication of the characterization and consideration of model uncertainty in the process.

Oftentimes, decision outcomes relate to the promulgation of rules aimed at specific sources of emissions, such as petroleum operations. In this chapter, two such decisions are considered to provide examples of the influences of modeling.

- Regulation IX, Rule 10: Nitrogen Oxides and Carbon Monoxide Emissions from Boilers, Steam Generators and Processes Heaters in Petroleum Refining ${ }^{94}$ implemented by the BAAQMD as mitigation for transport of emissions from the Bay Area to the Sacramento and San Joaquin Valley air basins.

- Rules 4305 (boilers, steam generators, and process heaters - Phase 2) and 4351 (boilers, steam generators, and process heaters - Phase 1) omitted by SJVUAPCD from the 94 Ozone Plan because the resultant emissions reductions were deemed unnecessary to meet the ozone NAAQS by 1999 in the SJV.

\footnotetext{
${ }^{94}$ Refer to www.baaqmd.gov/regs/rg0910.pdf.
} 
Figure VII-1: Ozone Production Isoquant

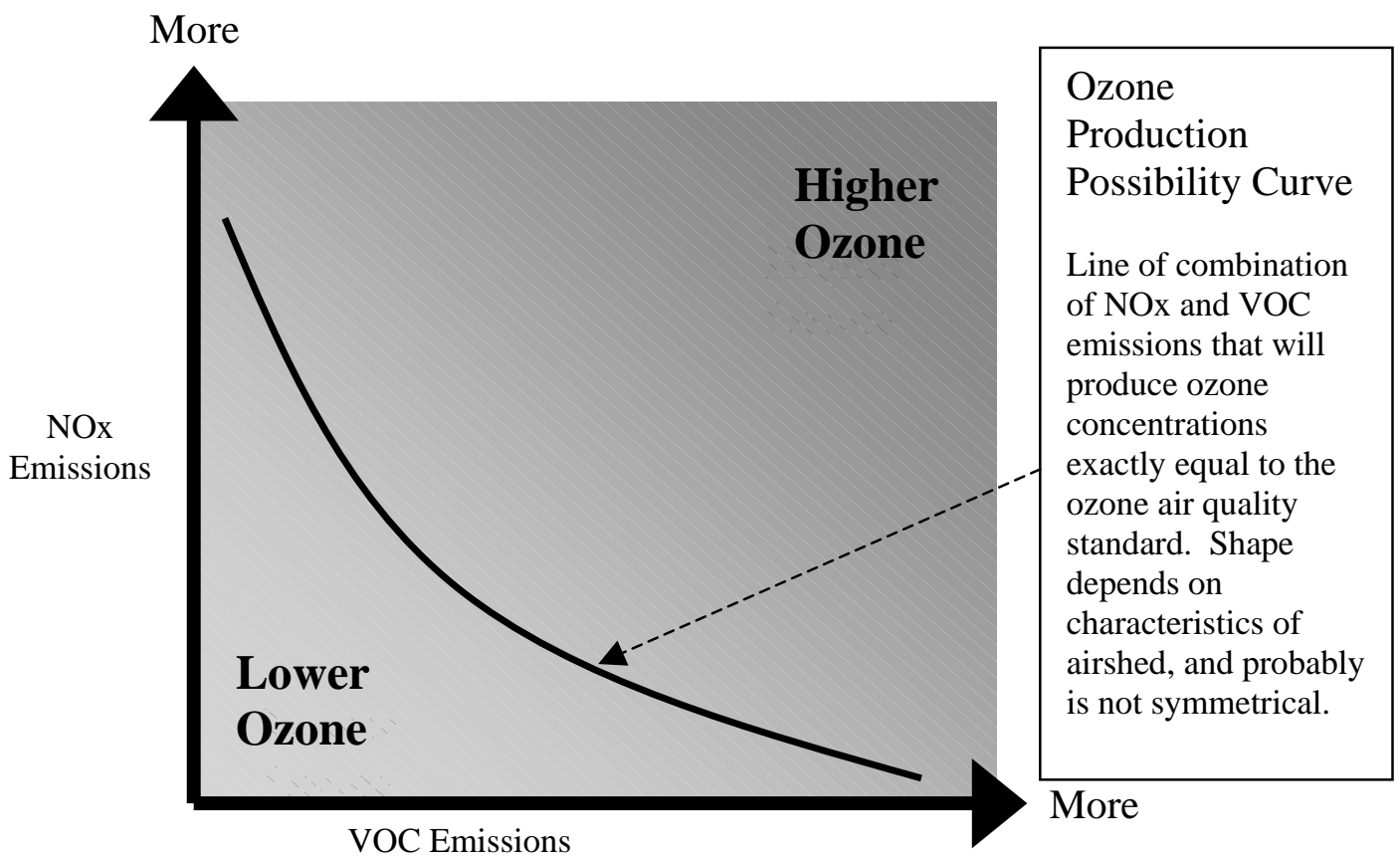

Figure VII-2: Ozone Reduction Isoquant

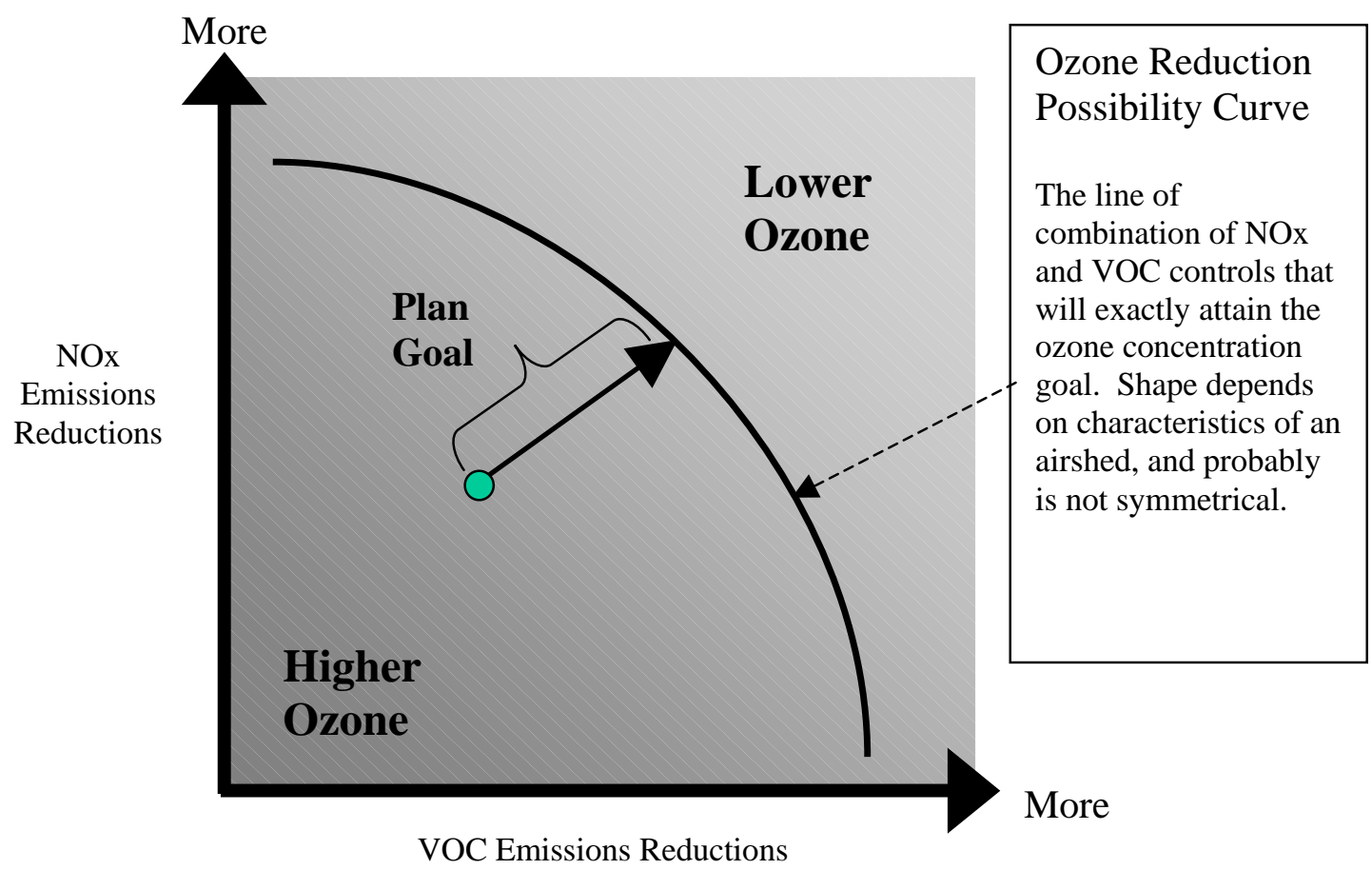

= current air quality conditions 


\section{Debates}

\section{Introduction}

The air quality problem in Central California can be described in many ways. It is the confluence of stories about population and economic growth, incomplete and insufficient scientific understanding, political will, industrial activity, jobs, people versus the environment, and environmental justice. This section presents four technical debates about air quality planning in Central California:

- Nonlinearity of ozone formation

- Choice of emissions control strategy

- Transport of air pollution from one district to another

- Representativeness of modeled ozone episodes

In the previous chapter, viewpoints about these issues are grouped into narratives. Here the arguments are laid out without focusing on the narratives so as to not confuse the matter. Clearly, though, the narratives are present in these arguments, as these and other stories were used to develop them. These issues indicate the different rationale for making emissions control decisions, and some of the associated uncertainties.

\section{Nonlinearity}

The chemical and physical processes involving ozone precursors, $\mathrm{NO}_{\mathrm{x}}$ and VOCs, that lead to high ozone concentrations are complex and often called "nonlinear". 95 Ozone formation involves counterintuitive relationships. Depending on atmospheric conditions,

\footnotetext{
${ }^{95} \mathrm{NO}_{\mathrm{x}}$ is shorthand for nitric oxide (NO) and nitrogen dioxide $\left(\mathrm{NO}_{2}\right)$. Combustion processes, such as fossil
} fuel burning, produce both VOCs and $\mathrm{NO}_{\mathrm{x}}$ emissions. Most nitrogen is emitted in the form of NO, but very 
as well as the temporal and spatial scales of analysis, reducing precursor pollutant emissions may decrease, increase or not affect ozone.

The primary photolytic cycle (PPC) is one way to understand the $\mathrm{NO}_{\mathrm{x}}$ and $\mathrm{O}_{3}$ relationship:

$$
\begin{aligned}
& \text { Reaction 1: } \mathrm{NO}_{2}+\mathrm{h} v \rightarrow \mathrm{NO}+\mathrm{O} \\
& \text { Reaction 2: } \mathrm{O}_{2}+\mathrm{O}+\mathrm{M} \rightarrow \mathrm{O}_{3}+\mathrm{M} \\
& \text { Reaction 3: } \mathrm{O}_{3}+\mathrm{NO} \rightarrow \mathrm{NO}_{2}+\mathrm{O}_{2}
\end{aligned}
$$

In words,

Reaction 1: Initiating step when the sun's energy (hv) causes a nitrogen dioxide $\left(\mathrm{NO}_{2}\right)$ molecule to disassociate into a fee oxygen atom $(\mathrm{O})$ and nitric oxide (NO). The free oxygen is called an oxygen radical because it is energized and highly reactive.

Reaction 2: Ozone-forming step when the oxygen radical combines with molecular oxygen $\left(\mathrm{O}_{2}\right)$. The $\mathrm{M}$ is a third molecule that must be present to facilitate ozone formation.

Reaction 3: Ozone-destroying step when nitric oxide reacts with ozone.

The PPC involves just three reactions amongst thousands involving hundreds of pollutants present in the atmosphere. Nonlinearity is due to reaction rates that depend quadratically on the concentrations of reacting pollutants. 
A good example of nonlinear ozone chemistry is, under some conditions, that reducing $\mathrm{NO}_{\mathrm{x}}$ emissions may increase ozone. As explained graphically below and shown in reaction 3 of the PPC, NO reacts with $\mathrm{O}_{3}$ by abstracting an oxygen atom. The products of this $\mathrm{O}_{3}$ "scavenging” reaction are $\mathrm{NO}_{2}$ and $\mathrm{O}_{2}$. $\mathrm{NO}$ converts $\mathrm{O}_{3}$ from its dangerous and oxidative form to inert molecular $\mathrm{O}_{2}$. Thus, the presence of NO may reduce the $\mathrm{O}_{3}$ concentration.

What is the role of VOCs? When VOCs react, their reaction products promote conversion of $\mathrm{NO}$ into $\mathrm{NO}_{2}$ without converting $\mathrm{O}_{3}$ to $\mathrm{O}_{2}$ via the PPC. With enough VOCs, the $\mathrm{O}_{3}$ concentration begins to increase. Otherwise, the three reactions of the PPC remain in balance and high ozone concentrations do not occur.

Another way to understand nonlinear ozone production is via the pseudo-steady state approximation (PSSA) (Seinfeld and Pandis 1998). The PSSA assumes the ozone concentration is in "steady state" which means the rate of change of $\mathrm{O}_{3}$ is zero. Mathematically, this is a derivative:

$$
\mathrm{d}\left[\mathrm{O}_{3}\right] / \mathrm{dt}=\mathrm{k}_{2}[\mathrm{O}]\left[\mathrm{O}_{2}\right][\mathrm{M}]-\mathrm{k}_{3}[\mathrm{NO}]\left[\mathrm{O}_{3}\right]=0
$$

Equation 4 says,

The change in ozone over a small period of time is equal to zero. ${ }^{96}$

Rearranging equation 4 yields,

$$
\left[\mathrm{O}_{3}\right]=\mathrm{k}_{2}[\mathrm{O}]\left[\mathrm{O}_{2}\right][\mathrm{M}] / \mathrm{k}_{3}[\mathrm{NO}]
$$

\footnotetext{
96 The brackets, [ ], are simply reminders that the chemical formulas indicate concentrations. A lowercase $\mathrm{k}_{\mathrm{n}}$ indicates the reaction rate; where the $\mathrm{n}$ indicates the reaction. The rates of reactions 1,2 and 3 in the primary photolytic cycle are represented by $\mathrm{k}_{1}, \mathrm{k}_{2}$ and $\mathrm{k}_{3}$, respectively. The units of $\mathrm{k}$ depend on the number of different molecules reacting. The values of $k$ depend on sunlight and temperature, but can be
} 
Assuming that reactions 1, 2 and 3 happen at roughly the same rate, reactions 1 and 2 can be rewritten as,

$$
[\mathrm{O}]=\mathrm{k}_{1}\left[\mathrm{NO}_{2}\right] / \mathrm{k}_{2}\left[\mathrm{O}_{2}\right][\mathrm{M}]
$$

Substituting equation 6 into equation 5 yields the photostationary state relation (PSSR):

$$
\left[\mathrm{O}_{3}\right]=\mathrm{k}_{1}\left[\mathrm{NO}_{2}\right] / \mathrm{k}_{3}[\mathrm{NO}]
$$

The PSSR shows how ozone depends on whether $\mathrm{NO}_{\mathrm{x}}$ emissions remain as NO or convert to $\mathrm{NO}_{2}$. Reducing $\mathrm{NO}$ will increase ozone. However, $\mathrm{NO}_{\mathrm{x}}$ is typically emitted as $\mathrm{NO}$, but converts quickly to $\mathrm{NO}_{2}$. Even if $\mathrm{NO}$ scavenges $\mathrm{O}_{3}$ near where it is emitted, it may lead to higher ozone concentrations downwind as atmospheric conditions change and the balance of pollutants shifts from more $\mathrm{NO}$ to more $\mathrm{NO}_{2}$. The PSSR simplification, however, can be dec

The isopleth concept introduced in the previous section is a tool used commonly for studying how the peak ozone concentration depends on precursor emissions. It shows the relationship between initial precursor emissions (or initial concentrations of precursors) and peak ozone.

Figure VII-3 is an isopleth from the Bay Area’s most recent ozone attainment demonstration plan (01 Ozone Plan). ${ }^{97}$ It indicates the amount of $\mathrm{NO}_{\mathrm{x}}$ and VOCs controls necessary to meet the ozone standard. The BAAQMD used it to argue that the

assumed to be constant for the time periods considered in the discussion of PSSA because temperature and sunlight do not change dramatically over a period of minutes.

${ }^{97}$ The isopleth diagram is an exact replica of the one shown in the Bay Area's 1999 ozone attainment plan. Thus, it shows the full range of emissions from zero to 752 and 681 tons per day of $\mathrm{NO}_{\mathrm{x}}$ and $\mathrm{VOC}$ emissions, respectively. But no simulations were done for reductions greater than 80 percent of either 
region is currently at point $\mathrm{A}$ and needs to get to the red point to exactly meet the ozone NAAQS standard. As emissions decline, ozone is presumed to decline from point A toward the origin. The red point is on the $124 \mathrm{ppb}$ ozone contour, which equals $120 \mathrm{ppb}$ (i.e., the ozone NAAQS) when rounded down.

The uncertainties associated with this isopleth are myriad and significant. For now, it is sufficient to note that Figure VII-3 is a very uncertain picture of ozone sensitivity in the Bay Area. Despite the uncertainties, the BAAQMD's isopleth corroborates other research indicating that $\mathrm{NO}_{\mathrm{x}}$ controls are not only ineffective, but might actually worsen air quality locally and make it tougher to meet the standard. Note the shape of the contours. If $\mathrm{NO}_{\mathrm{x}}$ emissions remain at 752 tons per day, VOC emissions need only be reduced to approximately 570 tons per day (tpd) to reach the contour line representing 124 ppb ozone. Reductions of $\mathrm{NO}_{\mathrm{x}}$ are planned, however, so VOCs must be reduced all the way down to 524 tpd.

pollutant, so the lower corners should not be depicted. 
Figure VII-3: BAAQMD Ozone Isopleth for Livermore (BAAQMD 2001a)

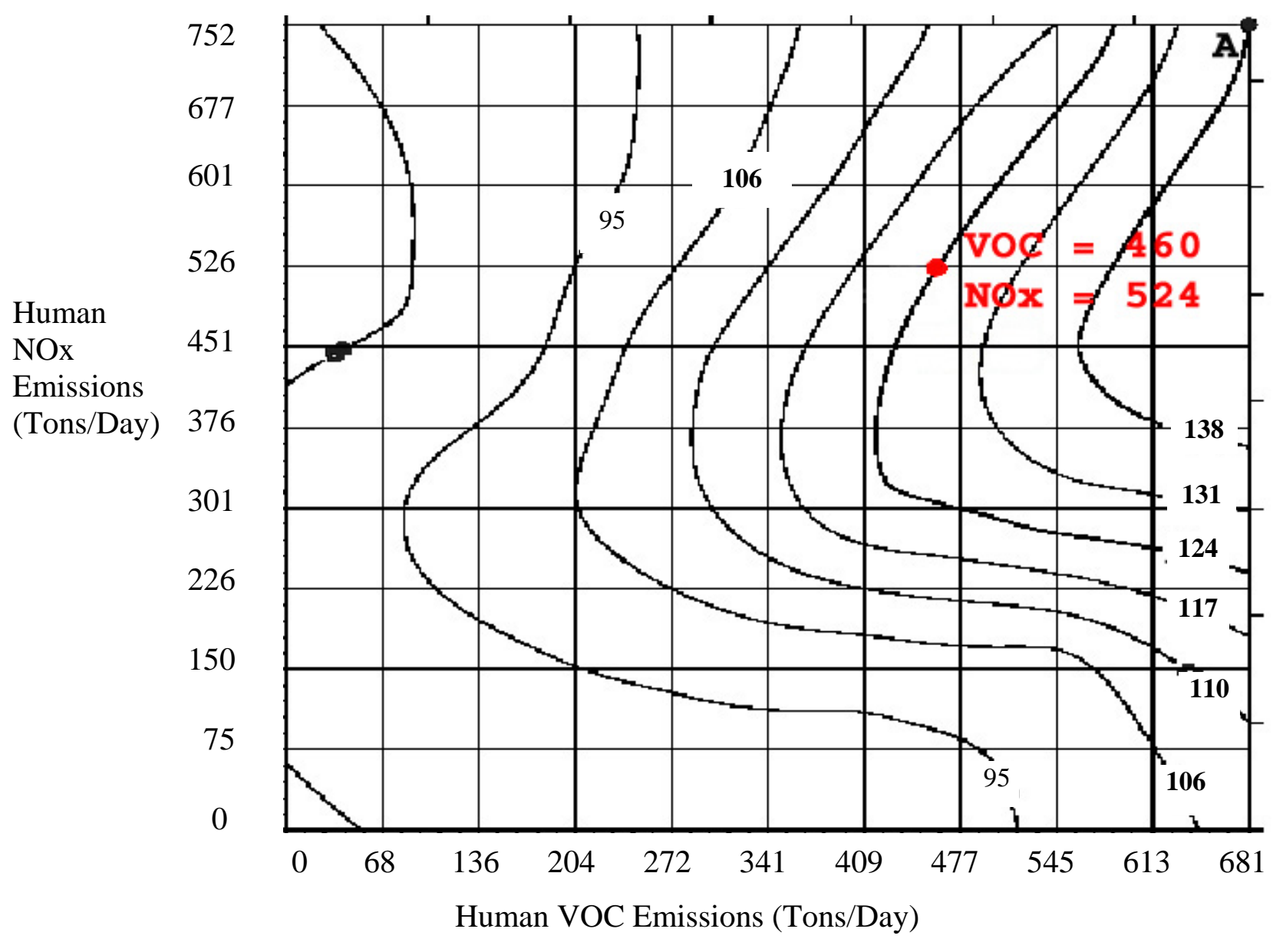


Planners and scientists alike recognize that ozone response to emissions changes may be counterintuitive. The BAAQMD's isopleth was presented in their most recent ozone attainment plan along with other evidence that their plan will succeed (BAAQMD 2001a). Nonetheless, it appears to be difficult to move away from the intuition associating emission reductions with linear declines in ozone pollution. For example, air quality attainment plans continue to use the "rollback" method as partial evidence that emissions controls will yield acceptably clean air. In discussing the rollback approach, the BAAQMD (2001a) wrote,

This is normally applied to non-reactive pollutants and is not considered a reliable quantitative analysis tool for ozone. Nonetheless, it is presented here to provide context and perspective....The linear rollback procedure is not the preferred method of analyzing pollutants formed (like ozone) through complex atmospheric chemistry. But it does, in this case, provide a perspective on the degree of nonattainment to be addressed in the Plan.

If the linear rollback perspective is not reliable, then why does the BAAQMD use it? Policy makers defend the linear perspective as a coarse policy response to a host of air quality concerns in the face of uncertainty. ${ }^{98}$ With the approval of CARB and EPA, the BAAQMD presented arguments based on linearity even though it acknowledges that the approach is not the "preferred method." In this case, the rollback approach was used even though it was known by technical experts to be inaccurate. For the lay public reading the BAAQMD's plan, however, the above disclaimer provides is all they have to understand the complexity of the science. This is an example of the incomplete treatment of the uncertainties.

\footnotetext{
${ }^{98}$ See quotes presented in Narratives discussion in Chapter IX.
} 
Experts seeking more precise air pollution control strategies to address ozone, such as scientists representing industrial sources, note the need to consider nonlinearity. ${ }^{99}$ The difference between a simple, linear view of ozone air quality and the more complex representation of nonlinearity is an indicator of the degree of understanding of the ozone problem and an important difference between air quality experts and laypersons.

The nonlinearity of ozone is also a crux point by which the two dominant narratives differentiate. In cases where a dual-precursor emissions control strategy is deemed a priori to be the policy option of choice, the simple linear relationship provides an adequate construct for associating controls with air quality improvements. This policy position is synonymous with the feasibility narrative.

Those seeking a more precise control path, using the science/efficiency narrative, may argue for a control strategy focused on a single pollutant, and may do so using the nonlinear relationship. In this case, the regulated industry forwards this argument. Public agents who support a dual control strategy do not benefit from explicit description of the nonlinear relationships.

Nonlinear atmospheric chemistry is an aspect of the planning problem that facilitates the opportunistic use of uncertainty. Depiction of a simple linear relationship between precursor emissions and either ozone or PM concentrations supports a policy of reducing all emissions, rather than the limiting reagent. Use of the "rollback" method in Ozone Attainment Demonstration Plans is one such example.

\footnotetext{
${ }^{99}$ Depicting nonlinear ozone formation using an isopleth also involves uncertainties. The ration of $\mathrm{NO}_{\mathrm{x}}$ to VOCs varies spatially and temporally. If emissions are underestimated, it introduces bias into the simulated effects of emissions controls. The limitations of isopleths are discussed in Chapter XII - Uses of Models and Uncertainty.
} 


\section{3. $\mathrm{NO}_{\mathrm{x}}$ versus VOCs Limitation}

In the presence of adequate sun and heat, either precursor pollutant, $\mathrm{NO}_{\mathrm{x}}$ or VOC, may be the limiting reagent for ozone formation. An emissions control "strategy" refers to the choice to control both or one precursor preferentially. A complete strategy will also include where, when, and, of course, how much to control.

The regulated community and scientists at the BAAQMD view the available evidence as indicating the need for a control strategy in the Bay Area that focuses on reducing VOC emissions. The isopleth in Figure VII-3 supports the conclusion that Bay Area ozone formation is VOC-limited. In general, areas below the ridgeline of the ozone contours are $\mathrm{NO}_{\mathrm{x}}$-limited. Areas above the ridgeline indicate a higher ratio of $\mathrm{NO}_{\mathrm{x}}$ to VOCs and, as such, VOC-limited regimes. However, to conclude based on only the isopleth would require accepting it as representative of all places and conditions in the Bay Area where high ozone occurs. It would also require adequate confidence that the estimate of emissions is sufficiently accurate so that the sensitivities shown are observed in the real atmosphere. The implications of these assumptions are discussed in detail in the next chapter.

Other evidence supports the finding that the Bay Area is VOC-limited. For example, anthropogenic emissions of $\mathrm{NO}_{\mathrm{x}}$ and VOCs estimated for the Bay Area in 2000 were 554 and 658 tons per day, respectively. In addition, an estimated 300 tons per day of VOC emissions are estimated from biogenic sources. This suggests a ratio of 1 ton $\mathrm{NO}_{\mathrm{x}}$ for each 1.3 tons of VOC emissions. This ratio is indicative of a VOC-limited regime (Seinfeld and Pandis 1998). 
The preferred control strategy for the SJV is less obvious. It is not clear if ozone formation in the region is limited by available $\mathrm{NO}_{\mathrm{x}}$ or VOC. It might be both, depending on the time and location. Rural areas tend to be $\mathrm{NO}_{\mathrm{x}}$-limited (Seinfeld and Pandis 1998). During the development of the 94 Ozone Plan, only field observations and the SARMAP modeling results were available to inform the control strategy choice. These modeling results, and the SJVUAPCD response to them, are discussed further in the next chapter. Today the issue remains uncertain. Observation-based modeling using data collected during the CCOS study identified both $\mathrm{NO}_{\mathrm{x}}$ and VOC-limited episodes in the SJV (Blanchard 2001). Additional research suggests that VOC reductions will have only modest impacts on 8-hour ozone extensive $\mathrm{NO}_{\mathrm{x}}$ reductions, and that $\mathrm{NO}_{\mathrm{x}}$ reductions on the order of 70-90 percent of emissions levels in 1999 may be necessary to meet the 8hour ozone standard in Central California (Reynolds et al. 2002). Thus, the current thinking is that the rural areas will require $\mathrm{NO}_{\mathrm{x}}$ controls, whereas urban areas need to continue to reduce VOC emissions. These research results are not definitive; uncertainties remain about, for examples, the estimate of biogenic emissions and the treatment of the differential ozone forming potential of VOC species.

The control strategy in the SJV is confounded by concerns about particulate matter. Studies using data collected as part of CRPAQS indicate that ammonium nitrate formation is limited by the availability of nitric acid $\left(\mathrm{HNO}_{3}\right)$ because ammonia $\left(\mathrm{NH}_{3}\right)$ is plentiful (Pun and Seigneur 1998). There is controversy about whether a $\mathrm{NO}_{\mathrm{x}}$ or VOCs control strategy is thus indicated. Although this would indicate the need for a $\mathrm{NO}_{\mathrm{x}}$ control strategy to reduce $\mathrm{PM}_{2.5}$ mass concentrations, evidence suggests that nitric acid formation is actually limited by VOC. However, significant gaps in current knowledge 
remain (Pun and Seigneur 1998). The NARSTO PM Assessment summarized the situation in the SJV as,

Strategies for reducing ozone during the summary may focus on $\mathrm{NO}_{\mathrm{x}}$ emission reductions since ozone formation within the SJV is then $\mathrm{NO}_{\mathrm{x}}$ limited. Reduction of $\mathrm{NO}_{\mathrm{x}}$ may not be the best course of action for reducing particulate nitrate in the possibly VOC-sensitive wintertime condition. Box model simulations indicate that $\mathrm{NO}_{\mathrm{x}}$ reductions may have the counterintuitive effect of increasing particulate nitrate formation during winter. Therefore, coordinated efforts will be required to formulate control strategies beneficial to both ozone and PM air quality (NARSTO 2003, page 10-13).

Inertia and divided authority may hinder attempts at a coordinated pollutant control strategy. For example, motor vehicle emissions standards are set at the state and federal levels and they call for declining emissions of both precursors. It may also be hard to specify the location of reductions. Non-point, area emissions sources will need to be abated to meet standards in the SJV. Both area and mobile emissions are spread throughout the region. Although vehicular emissions can be reduced, it is not possible to pick the location of the reductions, except that they will be concentrated on roadways.

Narratives discussed previously are demarcated, in part, by opinions about the necessity for and capability to define a $\mathrm{NO}_{\mathrm{x}}$ or VOCs control strategy. One view is that an important use of models is to prescribe a specific control strategy. Others consider models incapable of such precision. The capability and desirability of using modeling for this purpose is one crux point for studying uses of science to inform planning.

\section{Inter-district Transport}

As summarized in the previous section, the California Clean Air Act requires CARB to assess the impact of transported emissions on areas with poor air quality. Findings were initially published in a 1991 CARB report (CARB, 1990). The Western States 
Petroleum Association responded by contracting with Systems Applications, Inc. to critique and question the scientific basis of the findings relating Bay Area emissions to SJV air quality (Kessler 1990). In addition, technical staff at Chevron, working with WSPA, objected to CARB findings and called for reliance on modeling to assess transport (for examples, see Ziman 1993a; Ziman 1993b).

The transport assessment has been updated every three years. In 1994, SARMAP modeling was used as the basis for CARB's finding the Bay Area to be at times an overwhelming or significant contributor of upwind emissions to downwind violations of ozone standards in the SJV. Based on the modeling, CARB concluded that peak ozone in the north, central and south SJV would decline by 27, 10 and 7 percent, respectively, if Bay Area and Sacramento produced no emissions (Boyd 1994). The findings were published in SJV newspapers (Mearer 1994) and a SJVUAPCD press release, thereby imbedding the numbers into public thinking. The petroleum industry in the Bay Area again objected to the scientific bases of the findings, arguing that the episode modeled may not be representative of typical transport conditions and that the modeling method, which relied on setting emissions from the Bay Area and Sacramento regions to zero, may perturb the model beyond what is justified by model formulation (Ziman 2003). Nonetheless, CARB's finding was reaffirmed in the most recent transport assessment (CARB 2001c).

Uncertainties notwithstanding, the modeling provided hard numbers to decisionmakers, politicians and the public. The petroleum industry accepted that idea of transport, but was uncomfortable with CARB's quantification - 7, 10 and 27 percent given the uncertainties associated with the modeling study. In addition to modeling, 
CARB compared the magnitude of emissions in the Bay Area with those in the SJV, as well as prevailing wind patterns.

The transport assessment led the BAAQMD, under the direction of CARB, to promulgate Regulation IX, Rule 10: Nitrogen Oxides and Carbon Monoxide Emissions from Boilers, Steam Generators and Processes Heaters in Petroleum Refining. ${ }^{100}$ The petroleum industry objected to this rule on the grounds that reducing $\mathrm{NO}_{\mathrm{x}}$ emissions in the Bay Area would be very costly, possibly degrade ozone air quality locally (see Nonlinearity discussion above) and would yield little benefit downwind (for example, see WSPA 1996). The industry offered a counter-proposal to control $\mathrm{NO}_{\mathrm{x}}$ emissions directly in the SJV. Interactions between stakeholder, and eventual legal actions, are the focus of detailed analysis later in this chapter. It is an example where modeling evidence was the center of a dispute about what emissions to control and where to control them.

The story of interbasin transport and Bay Area $\mathrm{NO}_{\mathrm{x}}$ controls does not end with the refineries. Most recently, the SJVUAPCD filed a lawsuit alleging that EPA has failed to protect air quality in the SJV because it is not requiring the Bay Area to implement an enhanced vehicle emissions inspection program (API 2002). One justification for the lawsuit is the CARB transport assessment.

Assessments of transport are constrained by available measurement data, notably the paucity of actual days observed. Soon, however, capability may exist to model an entire year. Although computing power is currently adequate, there are insufficient observational data to support such modeling. As currently designed, routine monitoring

${ }^{100}$ Refer to www.baaqmd.gov/regs/rg0910.pdf. 
networks in the Bay Area and SJV are too sparse to provide needed input data. With adequate databases and subsequent modeling, more definitive statements will be possible about the degree and regularity of Bay Area emissions’ influence on the SJV.

\section{Representativeness of Modeled Episodes}

Modeling studies are typically based on one or a few ozone events (i.e., “episodes”) (Roth et al. 1997). Modeling in support of air quality planning for Central California, using SARMAP, simulated one ozone episode occurring August 3 to August 6 in 1990. Modeling by the BAAQMD to support local ozone planning, and to produce the isopleth shown in Figure VII-3, is based on one 1989 episode. These episodes do not represent the myriad conditions that might lead to high ozone or PM.

Meteorological conditions in the Bay Area and the SJV are quite variable. So too, many argue, should be control strategies. The different precursor regimes causing high ozone in the SJV indicate spatially and temporally specific control needs. The SJVAQS identified at least four meteorological regimes in SJV (Lehrman et al. 1998). Although high PM can occur in the SJV during both the fall and winter, they have very different sources. Fall PM is largely due to windblown dust, whereas wintertime PM has a larger fraction of secondary PM, notably ammonium nitrate. Consequently, $\mathrm{NO}_{\mathrm{x}}$ and VOCs control needs will vary temporally as well as spatially.

The science/efficiency narrative says modeling can and should be used to examine a representative sample of air quality conditions that lead to unacceptable ozone concentrations. Modeling every day of an ozone season, or multiple years of ozone seasons, for recently observed episodes, will address directly concerns about the 
representativeness of episodes studied thus far. The feasibility narrative does not object to this idea, but questions its utility if all feasible controls are currently in place or called for in existing plans.

\section{E. Decision Outcomes}

In the first section of this chapter, the regulatory impetus for air quality control decision-making was summarized. The second section introduced the decision space using a decision heuristic, the ozone isopleth. Then some of the debates associated with control decisions were summarized and discussed, while drawing upon the feasibility and science/efficiency narratives. In this final section, actual control decisions are noted, with special emphasis on decisions about requiring $\mathrm{NO}_{\mathrm{x}}$ controls on major refineries and steam generators used for petroleum production.

\section{1994 San Joaquin Valley Ozone Attainment Plan}

The 94 Ozone Plan set as targets for 1999 the reduction of 40 percent of 710 tons per day of $\mathrm{NO}_{\mathrm{x}}$ emissions and 38 percent of 505 tons per day of $\mathrm{VOC}$ emissions, as inventoried for 1990. Modeling indicated that this level of reductions was necessary to assure compliance with the ozone NAAQS by 1999. Most of the reductions were already included in the District’s 1991 Air Quality Attainment Plan. ${ }^{101}$ Additional reductions of 37 tons per day of both $\mathrm{NO}_{\mathrm{x}}$ and VOCs were called for in the 1994 plan.

The modeling demonstration showing attainment of the ozone NAAQS by 1999 depended on many key assumptions, notably the current and projected emissions

\footnotetext{
${ }^{101}$ The 1991 plan was a Clean Air Plan pursuant to the requirements of the California Clean Air Act. That plan contained "all feasible control measures" and was approved by CARB. It was produced before the unification of the SJV air districts into the SJVUAPCD, the initiation of the SJVAQS, and was not reliant on modeling.
} 
inventory, boundary conditions, and the representativeness of meteorological conditions simulated. The anthropogenic VOC emissions inventory was known to be underestimated; notably missing evaporative emissions from motor vehicles, additional oil and gas production sources, and off-road farm equipment emissions (e.g., diesel engines used to pump water). Refer to Chapter IV for a detailed discussion of emissions sources in the SJV. Only one ozone episode was simulated: an event that occurred in early August of 1990. Furthermore, upwind boundary conditions were anticipated to improve dramatically by 1999 thanks to emissions controls implemented in the Bay Area. Per Jones' comment presented in the previous chapter, modeling did not show attainment until assumed future boundary conditions were lowered, presumably to reflect the anticipated benefits of upwind emissions controls in the Bay Area.

Despite the known, but not quantified, uncertainties associated with the modeling system, as well as performance evaluation that was incomplete and ongoing, the modeling was used to justify the omission of some available emissions controls. The plan did not call for implementation of all feasible measures. Specifically, it exempted steam generators in western Kern County from Rules 4306 and 4702. Relying on EPA’s definition of Reasonable Available Retrofit Control Technology (RACT), the rules involved the same control technology as called for in the BAAQMD’s Regulation IX, Rule 10. Had they been implemented district-wide, 7.6 and 12.4 tons per day, respectively, would have been achieved from rules 4306 and 4702. No information is provided to indicate what portion of those reductions would have been attributed to westside sources. Nonetheless, the combined 20 tons per day is over half of the $\mathrm{NO}_{\mathrm{x}}$ 
emissions reductions from new controls called for in the 94 Ozone Plan. The explanation

provided in the plan for the omissions is,

A model run showing attainment in 1999 has been prepared without taking credit for reductions from westside oilfield $\mathrm{NO}_{\mathrm{x}}$ emissions in the area west of Interstate 5 in Kern, Kings, and Fresno Counties, (the westside). The District is not taking credit for reductions in westside oilfield $\mathrm{NO}_{\mathrm{x}}$ emissions for control measures identified in the Attainment Demonstration Plan. Proposed $\mathrm{NO}_{\mathrm{x}}$ Rules 4306 and 4702, when submitted for inclusion in the District's SIP, will not propose applicability for westside oilfield $\mathrm{NO}_{\mathrm{x}}$ sources. (SJVUAPCD 1994, page 4-2).

Ziman of Chevron said of this decision,

We made these runs even though the San Joaquin Valley Air District was adamantly opposed to it. The long and short of the runs were that there was no significant impact of west side $\mathrm{NO}_{\mathrm{x}}$ controls on ozone concentration in the east side.

We had an idea of somewhat why. When we had looked at tracer studies in 1985, we didn't see flow from the west side getting to the east side. The Valley seems to bifurcate in some respects....

So we applied for 182(f) [exemption from $\mathrm{NO}_{\mathrm{x}}$ controls]. And CARB agreed with it, which was very interesting.

Ziman is referring to section 182(f) of the FCAA, which allows major stationary sources to opt out of $\mathrm{NO}_{\mathrm{x}}$ controls if it can show, using models, that "net air quality benefits are greater in the absence of reductions of oxides of nitrogen (EPA 1992b). Though CARB and the SJVUAPCD did allow for the 182(f) application, they really had no choice due to the language of the law. Scheible , CARB Deputy Executive Officer, said,

I was against the exclusion of the controls because I thought they were necessary for broader air quality reasons.

Through their sponsorship of the SJVAQS, the regulated community had earned the right to define some modeling scenarios. Further impetus was provided by section 182(f) of FCAA. Consequently, the SJVUAPCD and CARB lacked control over some of the 
decision-making. By defining the problem through model scenario design, petroleum interests were able to persuade the SJVUAPCD to omit control requirements from the 94 Ozone Plan. This is an example of using the model to support a desired control decision outcome. Before the modeling, the regulated community's logic was that west side $\mathrm{NO}_{\mathrm{x}}$ controls do not matter for ozone in SJV's east side. This hypothesis was based on research done previously in the SJV (Blumenthal et al. 1985). The SARMAP modeling corroborated earlier research, even though it was quite uncertain. Was this episode representative of all of the meteorological conditions that led to high ozone in the SJV? The answer to this question was uncertain in the 1990's, and remains so today.

\section{CAPs}

Ultimately, controls were implemented to reduce $\mathrm{NO}_{\mathrm{x}}$ emissions from oilfields in the westside of the SJV. They were part of the strategy to pursue "all feasible measures" as required by the CCAA (as well as $\mathrm{PM}_{10}$ NAAQS planning requirements) However, from industry's perspective, this result was better than including the $\mathrm{NO}_{\mathrm{x}}$ controls in the 94 Ozone Plan for two reasons. First, control implementation was delayed. Second, the controls were not "federalized".

Controls are expensive. Any delay in control implementation means delaying of control costs. Delays are quite valuable, since time is money and the costs are on the order of hundreds of millions of dollars. Financial concerns are exacerbated when required emissions controls have uncertain, and potentially negative, impacts on air quality. 
The bureaucratic hassle and threat of federal court involvement is sufficient impetus for the regulated community to try to avoid the "federalization" of emissions controls. The status of controls as federalized, or not, has bearing on the penalties associated with failure to implement required controls, and the legal process necessary to undo or change the rules. Essentially, it is the regulated community's best interest to avoid the need for EPA or court approvals for rule changes wherever possible.

An outcome of the CAP effort is that the state standard is never met. In areas, such as the Bay Area and the SJV where a 120 ppb ozone threshold has thus far been an ambitious goal, achieving the state standard of $90 \mathrm{ppb}$ is deemed impossible. Nonetheless, the CAPs provide the impetus to implement “all feasible” controls not otherwise justified through attainment demonstration modeling conducted for an ozone plan.

\section{Transport Assessment and Mitigation}

Initially, CARB concluded that the Bay Area was, at times, a significant contributor to state ozone standard violations in the SJV (CARB 1990). In the subsequent assessments of 1993 and 1996, however, CARB found the Bay Area to be, at times, an “overwhelming”102 emissions source for SJV ozone standard violations (CARB 1993).

Figure VII-4 shows arrows that depict results of the California Air Resources Board's assessments of transport. CARB finds that BAAQMD, SMAQMD and SJVUAPCD are transport partners, with emissions in the BAAQMD carried on winds to both the

\footnotetext{
102 The term "overwhelming” is specifically required by the language of the CCAA. See discussion of Clean Air Laws in Appendix B.
} 
SMAQMD and SJVUAPCD, and the SJVUAPCD and SMAQMD transporting pollutants to each other.

Figure VII-4: Inter-district Transport

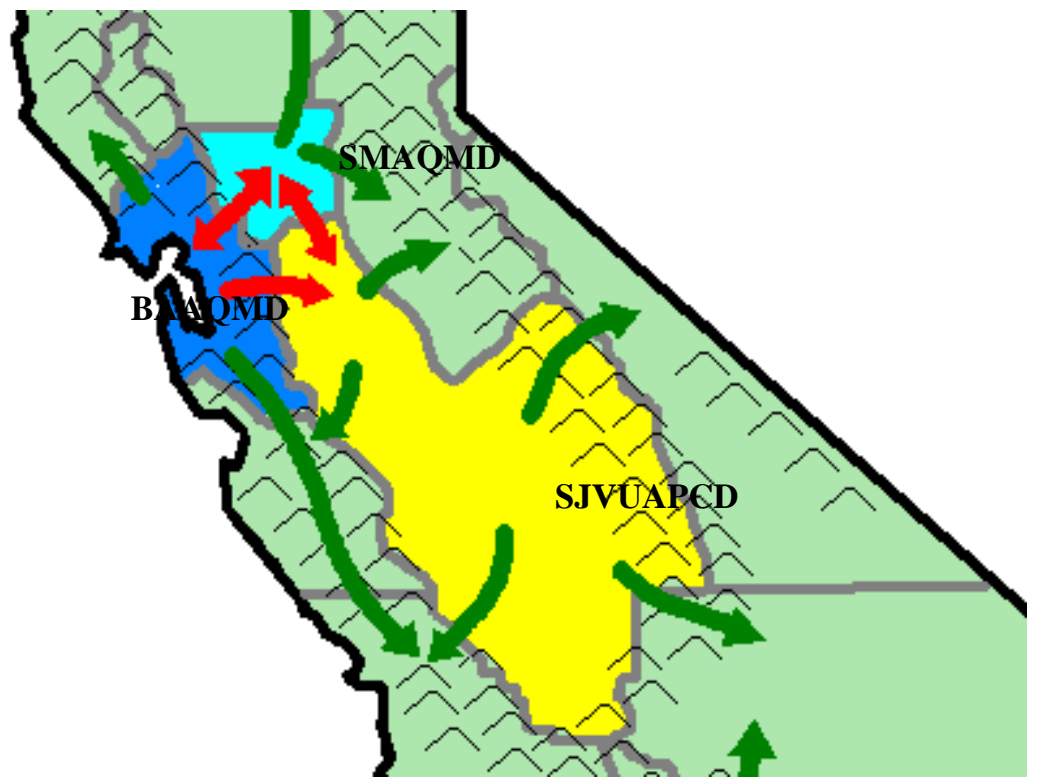

CARB based their findings on SARMAP modeling, simulating the same August 3-6, 1990, ozone episode used for the 94 Ozone Plan. The simulation involved zeroing out emissions from the SJV to determine if emissions from the Bay Area and Sacramento were sufficient to produce ozone concentrations above the $\mathrm{O}_{3}$ NAAQS. In addition, trajectory analyses and comparisons of the magnitude of emissions in the two air districts were used to support conclusions. Of course, all three of these methods - modeling, trajectories, and emissions comparisons, have significant limitations and uncertainties. Yet, they were the best available information. In defending their finding, CARB wrote,

Air quality modeling for that day with all anthropogenic emissions removed from the San Joaquin Valley clearly demonstrates overwhelming transport into a large portion of the northern Valley. (CARB 1996a) 
The report noted that CARB staff found two ozone exceedences at Crow's Landing and Tracy on August 1 and August 9, 1995, "that were due to overwhelming transport.” The BAAQMD commented,

We believe that some of the transport-assessment approaches used by the CARB fail to take into consideration some important characteristics of ozone episodes and thereby can lead to invalid conclusions. Because the atmospheric dynamics associated with the ozone episodes are complex and incompletely known, we need an underlying conceptual model that helps guide our thinking...(CARB 1996a)

CARB responded,

The CARB believes the SARMAP is far beyond a conceptual model and is in fact the most sophisticated meteorological and air quality model available. The results from the SARMAP analyses provide the most thorough scientific transport assessment possible for the study of this transport couple...(CARB 1996a)

When the BAAQMD commented that they did no think that the analyses to date have demonstrated instances of overwhelming impacts, CARB stated,

Even with the use of the best models there are some uncertainties in any analyses. The knowledge and skills are improving all the time. The uncertainties, however, should not be used to invalidate or void the findings presented in the report. (CARB 1996a)

This interchange reveals how modeling is used to justify decisions and how the model uncertainties are both the bane and bounty of decision-makers and stakeholders. It demonstrates the complexity of the decision-making setting and associated debates. The BAAQMD expressed concerns about the uncertainties associated with CARB's analyses, notably the representatives of the episode studied by CARB. In retort, CARB said that they were using the best available tools to study the problem.

The uncertainties associated with the modeling, and the political use of those uncertainties, is exemplified by the BAAQMD’s promulgation of rules requiring 
selective catalytic $\mathrm{NO}_{\mathrm{x}}$ reduction on refineries in the Bay Area. Whereas modeling in support of the 94 Ozone Plan provided the justification for forgoing $\mathrm{NO}_{\mathrm{x}}$ controls on steam generators in the west side of the SJV, it provided CARB the ammunition to require implementation of the same control technology in the Bay Area. Specifically, through Regulation IX, Rule 10, five petroleum refineries would be required to install selective catalytic $\mathrm{NO}_{\mathrm{x}}$ controls on their boiler smokestacks. To the refiners, the rule meant spending an estimated \$360 million to install SCR to eliminate approximately 24 tons per day of $\mathrm{NO}_{\mathrm{x}}$ emissions.

The refiners facing these costs were keenly interested in the limits of the evidence offered by CARB. Most of that evidence took the form of modeling. WSPA and Chevron, with cooperation from the BAAQMD, attempted to use modeling to show that $\mathrm{NO}_{\mathrm{x}}$ controls would not only yield little downwind benefits in the SJV, but would worsen ozone in the Bay Area.

The BAAQMD had done its own modeling using a different modeling system than SARMAP to conclude that $\mathrm{NO}_{\mathrm{x}}$ controls would not be beneficial locally (Martien et al. 1992; Martien and Umeda 1993). Executing the Urban Airshed Model, the BAAQMD simulated an ozone event observed in September, 1989 to estimate population exposure to concentrations of ozone greater than $95.5 \mathrm{ppb}$ both with and without the reductions anticipated from refinery $\mathrm{NO}_{\mathrm{x}}$ controls. In an internal memo, Dr. Dave Fairley, a statistician at the BAAQMD, wrote that ozone exposure was thirteen percent higher with $\mathrm{NO}_{\mathrm{x}}$ controls. This finding supported other indications that $\mathrm{NO}_{\mathrm{x}}$ controls would cause ozone to increase locally. Fairley (1996) was straightforward about the study and its associated uncertainties when he wrote, 
It should be kept in mind that this analysis is based on one model run for one day. The model predicts state excesses for only a tiny fraction of the grid-square-hours, and most of these lie in lightly populated areas. It would be desirable to obtain modeling results for the other days with high ozone and varying meteorology to provide reliable estimates of the overall effects of the $\mathrm{NO}_{\mathrm{x}}$ rule.

In addition, WSPA contracted with Alpine Geophysics to calculate the same population exposure statistics used by Fairley, only this time using SARMAP and the August 3-6, 1990 ozone episode. The findings concurred with the BAAQMD study that there were no net benefits from $\mathrm{NO}_{\mathrm{x}}$ controls in terms of reducing population exposure (Ziman 1996b). Yet, Ziman expressed concern that CARB would be unwilling to accept other's modeling results, when he wrote,

There is a major question about the credibility of the work. If we did it without consulting with either the CARB or the BAAQMD about the scenarios, and how they were done, there is little reason to believe that the not-invented-here syndrome will not kick in, regardless of the quality of the work. We need to have them involved as we do the work, so that our emissions reductions are not questioned (Ziman 1996a).

Ultimately, WSPA convinced CARB technical staff to simulate the effects of Bay Area $\mathrm{NO}_{\mathrm{x}}$ controls using SARMAP. In a memo from Dr. Adjit Kaduwela (1996), a modeler at CARB, the results were summarized as :

- Richmond monitoring station is VOC-limited, and there will be a disbenefit from $\mathrm{NO}_{\mathrm{x}}$ controls.

- Livermore, San Jose and Pittsburgh isopleths show disbenefits to $\mathrm{NO}_{\mathrm{x}}$ controls, and benefits from VOC controls. These sites are downwind of the Bay Area's $\mathrm{NO}_{\mathrm{x}}$ emissions. Pittsburgh also has high $\mathrm{NO}_{\mathrm{x}}$ emissions from utility stacks nearby.

- Stockton, Fresno and Edison sites have closer to "traditional" isopleths, so their isopleths favor $\mathrm{NO}_{\mathrm{x}}$ reduction. 
Like Fairley and other modeling technicians, Kaduwela noted concerns about the uncertainties associated with the isopleths. Kaduwela's supervisor, Mr. Andrew Ranzieri, summarized the results and examined the differences between the BAAQMD and CARB modeling runs,

We believe that both simulations show a common trend: $\mathrm{The}_{\mathrm{NO}}$ control in a $\mathrm{NO}_{\mathrm{x}}$ rich area increases $\mathrm{O}_{3}$ concentration in the immediate downwind of that area and decreases the $\mathrm{O}_{3}$ concentration further down wind where RHC concentration is high. The disbenefit in BAAQMD's simulation extends up to the eastern edge of the Bay-Area air basin. However, CARB simulations show a disbenefit area extending into the Valley. (Ranzieri 1996).

Ranzieri attributes the slight differences in the results to assumptions about wind speed. Nonetheless, these admittedly uncertain modeling results were all pointing to the same conclusions that $\mathrm{NO}_{\mathrm{x}}$ controls in the Bay Area would not necessarily address transport concerns, and could make conditions worse locally. Reporting on the CARB's modeling results, Ziman wrote,

ARB Technical Staff and their management are becoming more comfortable with the results. We have yet to see how senior CARB management will respond...ARB simulations have not been released, and may not be depending on decisions by CARB management. All information is verbal (Ziman 1996a).

Ziman's comment speaks to concern about the control of information, as well as the divide between modelers and policy-makers at CARB. If decision-makers at CARB desire Bay Area $\mathrm{NO}_{\mathrm{x}}$ controls, they may be unwilling to release technical results that do not support the policy. Furthermore, Ziman notes that the "management" at CARB, namely the Executive Officers, control the dissemination of official modeling results. His concern was that modeling results would, in the words of Leong, "never see the light of day" because they did not support the feasibility policy. 
Making little progress with modeling, WSPA offered CARB a trade. In exchange for delaying implementation of Bay Area refinery $\mathrm{NO}_{\mathrm{x}}$ controls, WSPA would pay for alternative controls in the SJV . The hope was that the extra time would allow the science to further inform the Bay Area $\mathrm{NO}_{\mathrm{x}}$ control debate. Presumably, WSPA and Chevron believed that additional research would support their argument against $\mathrm{NO}_{\mathrm{x}}$ reductions in the Bay Area. This is an example of the science/efficiency narrative with all of its hopes.

Petroleum interests were also seeking legal remedies. WSPA's proposed trade was made after they filed a lawsuit against the BAAQMD. The lawsuit claimed that the proposed $\mathrm{NO}_{\mathrm{x}}$ controls rules would have significant adverse air quality impacts and, therefore, necessitated review under the California Environmental Quality Act. WSPA was willing to give up the lawsuit, however, if the BAAQMD agreed to the terms of a proposed trade. In exchange for delaying $\mathrm{NO}_{\mathrm{x}}$ control rules implementation for three years until 2005 "pending a scientific review of upwind and downwind impacts" (Reheis 1998), WSPA proposed to:

- Reduce $\mathrm{NO}_{\mathrm{x}}$ and VOC emissions by 6.1 tpd and 1.3 tpd, respectively, at a cost of $\$ 11$ million, by replacing old diesel agricultural water pump engines in Kern and Fresno Counties in the SJV.

- Enhance the SMAQMD’s SEED program by \$3 million to achieve up to 1.3 and 0.28 tpd reductions of $\mathrm{NO}_{\mathrm{x}}$ and $\mathrm{VOC}$, respectively, via the replacement of old diesel water pump engines.

- Provide low emission vans for the BART Links vanpool program, at a cost of $\$ 3$ million.

- Augment the Altamont Commuter Express (ACE) program with a \$2 million grant. 
- Fund a $\mathrm{NO}_{\mathrm{x}}$ air quality study to (1) determine effects of Bay Area $\mathrm{NO}_{\mathrm{x}}$ reductions on Bay Area and downwind standards attainment, (2) identify any necessary alternative emission controls; and, (3) conduct additional research and model development for other downwind Districts (e.g., SMAQMD) (1998).

At first brush, the proposal seemed to be a win-win scenario for all; WSPA called it a “win-win, no lose, risk free solution” (Reheis 1998). The SJV would get much needed emissions reductions locally, presumably to more directly improve its air quality. The Bay Area would not experience the anticipated disbenefits from local $\mathrm{NO}_{\mathrm{x}}$ controls. CARB would get ozone reduction in the SJV at the expense of Bay Area polluters. Finally, Bay Area refiners would spend far less money (\$22 million compared with \$360 million, see (Unknown 1998)) on controls that would be less detrimental to air quality in the Bay Area, while improving conditions in the SJV.

Others, however, objected to the proposal, so political pressure mounted in opposition to the trade. The SJV, Northern Sierra and Sacramento air quality management districts had significant concerns (for examples, see Crow 1997; Covell 1998; VanZam 1998; WSPA 1998). Senator Byron Sher (1998), who helped author the CCAA, wrote to urge the BAAQMD Board to reject the proposal, stating,

There is no technical flaw in the rule. It satisfies cost-effectiveness criteria. The control technology it represents - selective catalytic reduction - is required for all other major combustion sources in the state and has been successfully implemented at numerous sites. There is nothing unique about Bay Area refineries that compel a different compliance approach .

In his letter, Sher did not acknowledge the scientific evidence describing the complexity of transported pollutants, ozone formation, or the possibility of a $\mathrm{NO}_{\mathrm{x}}$ disbenefit. He noted cost-effectiveness and equity criteria, such as the fact that others 
have implemented the controls so Bay Area refiners should do so too. This type of thinking superceded or, at least, overshadowed the technical evidence.

Furthermore, decision-makers at the BAAQMD knew that the deal had negative public relations currency. In the eyes of a public not familiar with nonlinear atmospheric chemistry, the BAAQMD may have been perceived as caving in to industry, allowing polluters to forego local emissions controls. That is, without a deeper understanding of the science, the public may not accept that $\mathrm{NO}_{\mathrm{x}}$ emissions reductions actually make ozone more severe locally.

The SMAQMD, as another downwind receptor of Bay Area emissions, also objected to the WSPA proposal. In his letter to the SMAQMD, Mr. Norm Covell, the air district's Air Pollution Control Officer, concluded,

Given the magnitude of the emissions reductions from these refineries, uncertainties regarding the equivalent air quality benefits from the proposal and all the other reasons described above, this proposal is not considered to be in the best interest of the Sacramento Country residents (Covell 1998).

The SJVUAPCD faced very different concerns about WSPA's proposal that centered on the uncertainties. Mr. David Crow, Air Pollution Control Officer for the SJVUAPCD, sent a letter to CARB with a long list of questions. Many of the questions expressed concern that CARB may be making decisions "without sufficient scientific understanding", while others noted that the SJVUAPCD Board must be presented with solid answers, not just a theory and unanswered questions. Indicative of these concerns are questions, such as:

Can CARB establish for our Board that the small emissions changes being investigated are really reliable, considering the general uncertainties of the model, the large missing quantities of emissions that are now known to exist 
in the northern SJV and considering its own guidance on the level of emissions change that should be used to have confidence in a model's predictions? (Crow 1997)

Ultimately, CARB rejected WSPA's proposal, using uncertainty as the primary

justification. In a letter from Mr. Mike Kenny, Executive Officer, CARB backed away

from modeling results produced by its staff, saying,

WSPA believes the local $\mathrm{NO}_{\mathrm{x}}$ reductions they've proposed are equivalent to upwind refinery controls. WSPA has indicated that they used modeling work done by CARB to reach this conclusion. However, none of the model runs conducted by the CARB are adequate to estimate even the approximate affects of the WSPA alternative reductions on peak ozone in the urban areas of Fresno or Kern...

Just as Ziman had feared, CARB decision-makers disregarded the results of their own technical staff. They were using modeling uncertainties to do so. Kenny then expressed broader uncertainties not necessarily attributable to the modeling as rationale for not being able to support the proposal,

There is insufficient technical information to determine the feasibility of the WSPA proposal...In the near term, the WSPA alternative appears less certain than the refinery rules.... there are uncertainties over the number of engines that would need to be converted, the amount and location of air quality benefits, and whether there would be sufficient number of engines or participants. Finally, enforceability of the alternative approach is unknown. Thus, it is impossible to conclude that the proposal results in equivalent ozone benefits (Kenny 1998).

It appears that CARB discounts its own research, and rejects the WSPA proposal. There are myriad political and legal concerns might be cited for this. Ultimately, the trade would have conflicted with CARB's decision to require $\mathrm{NO}_{\mathrm{x}}$ controls on Bay Area refineries. Thus, uncertainty was at the heart of the debate, but its role and use derived from other political concerns. 


\section{F. Summary of Chapter X: Debates and Decisions}

This chapter lays out the decision requirements, decision spaces, and debates attending air quality control in Central California during the 1990’s. Three planning requirements provide the foci:

1. Ozone Attainment Demonstration Plans for ozone and PM NAAQS per the FCAA

2. Clean Air Plans for ozone per the CCAA

3. Transport assessment and mitigation per the CCAA

Four different aspects of the debates attending emissions control decisions are described: nonlinearity, control strategy choice, inter-district transport, and the representativeness of studied ozone episodes. Narratives defined in the previous chapter are used to add flesh to the debates, and to associate opinions with stakeholder groups.

The chapter ends with a discussion of the decisions actually made, and how models were used in the decision-making process.

Several findings are noteworthy:

- A primary constraint on decision making identified in the previous chapter was the availability of feasible control measures. The 94 Ozone Plan, however, did not call for implementation of all feasible measures. It exempted steam generators in western Kern County from $\mathrm{NO}_{\mathrm{x}}$ controls. Justification for this omission was provided by a SARMAP simulation.

- In preparing the 94 Ozone Plan, the decision-making authority of CARB and the SJVUAPCD was undermined when the regulated community - petroleum producers - exercised their right, based on Section 182(f) of the FCAA, to request specific model simulations. The simulation results undermined justification for requiring $\mathrm{NO}_{\mathrm{x}}$ controls on steam generators in western Kern County, which was counter to a policy of controlling all feasible emissions.

- If controls are seen as imminent, but are not supported by technical analyses, the regulated community may seek intermediate strategies to delay control 
costs and to avoid their federalization. In pursuit of these objectives, modeling was use opportunistically per the previous finding.

- Delayed implementation of controls serves another important objective for the regulated community, as articulated through the science/efficiency narrative. It gives the science time to inform decisions further. This was the expectation regarding refinery $\mathrm{NO}_{\mathrm{x}}$ controls in the Bay Area. Through first a lawsuit and then a proposed trade, WSPA and Chevron hoped the science would eventually make the case against the need for $\mathrm{NO}_{\mathrm{x}}$ controls. The stakes in the Bay Area were greater than in the SJV, since $\mathrm{NO}_{\mathrm{x}}$ controls were believed by some, including technical staff at the air districts, to make ozone worse locally, not better.

- Pertaining to $\mathrm{NO}_{\mathrm{x}}$ controls in the Bay Area, the science was overwhelmed by political concerns. CARB saw refinery $\mathrm{NO}_{\mathrm{x}}$ controls as part of their policy of controlling all emissions feasible. CARB used transport concerns, rather than local ozone air quality, as the impetus for controls. Thus, the SJVUAPCD came to have an interest in Bay Area $\mathrm{NO}_{\mathrm{x}}$ controls. The SJVUAPCD rallied political support to pressure CARB to require the controls. After first producing modeling results that did not support Bay Area $\mathrm{NO}_{\mathrm{x}}$ controls, the BAAQMD eventually chose not to risk being seen in the eyes of the public as willingly letting local refiners avoid controls. The BAAQMD, SJVUAPCD and CARB all had political reasons to want $\mathrm{NO}_{\mathrm{x}}$ controls. After first being the focus of debate, modeling evidence was obfuscated by invoking uncertainty concerns because it did not support a decision to control.

- In two instances, information control proved pivotal in the use of modeling results for decision-making. In the SJV, control over modeling scenario definition granted by the language of the FCAA gave the regulated industry the upper hand. For Bay Area $\mathrm{NO}_{\mathrm{x}}$ controls, CARB Executive Officers deemphasized modeling results produced by their technical staff. CARB questioned the reliability of the modeling and highlighted other uncertainties associated with WSPA's proposal to implement emissions controls on agricultural diesel engines in the SJV rather than $\mathrm{NO}_{\mathrm{x}}$ controls on Bay Area refineries. 


\section{CENTRAL CALIFORNIA PLANNING PART III - USES OF MODELS AND UNCERTAINTY}

\section{A. Introduction}

This chapter summarizes and extends conclusions of the previous chapters about Central California air quality planning during the 1990’s to make nine assertions about the roles of models and uncertainty.

The use of models is examined in terms of regulatory requirements and constraints on the processes identified by interview respondents. Findings pertaining to the ways models affect the planning process are illustrated by refinery $\mathrm{NO}_{\mathrm{x}}$ control decisions. The primary finding about models is that they do not delineate decisions, but do affect the process.

The role of uncertainty is summarized by examining discourses of uncertainty in the two dominant policy narratives - feasibility and science/efficiency. The narratives are critiqued to identify rhetorical uses of uncertainty, notably via the isopleth heuristic. ${ }^{103}$ Stakeholders are found to use uncertainty opportunistically to support their policy narratives.

Broader generalizations about modeling uncertainty are also presented. The assumption made by stakeholders about the implications of explicating uncertainties that it will mean the need for more controls - is identified and refuted. The communication and management of uncertainties throughout the decision-making

\footnotetext{
103 Although this research focuses on the use of modeling, it is important to note that narratives rely on more than the models to form opinions. The science/efficiency narrative uses observational data analyses and corroborative methods, such as sensitivity analyses, to develop opinions reinforced by modeling results. Comparatively, the feasibility narrative relies on a broad problem definition and heuristic approaches to manage uncertainty while de-emphasizes modeling results.
} 
hierarchy is also critiqued. Finally, the anticipated benefits of more rigorous and thorough model performance evaluation are discussed in light of findings.

The findings of this chapter indicate that there are formidable disincentives to generating, communicating or managing explicitly uncertainty information.

\section{B. Role of Models}

This section answers the question: How are models used in air quality planning?

\section{Decision-Making with Modeling}

Evidence presented in previous chapters, notably respondent statements, revealed that other constraints determined decisions, including time, legal requirements, and political will. Consequently, models were used to justify the a priori decisions of those in control of the modeling.

Speaking about his modeling work at CARB, Dr. Saffet Tanrikulu summed up the role of models in decision-making,

We're basically providing a piece of the puzzle to management. Management takes that information, and management has some other information. The policy is usually not made based upon the modeling information.

Whereas regulations, such as the FCAA, create requirements for using models, their actual use is not as might have been envisioned by those regulations.

\section{The Attainment Demonstration Myth}

The FCAA specifically requires modeling be used to show that planned controls will result in the attainment of NAAQS by a specified date. Strictly speaking, this “attainment demonstration” does not require that models be used deterministically to delineate controls. Rather, they need only show that planned controls will be sufficient 
by simulating the effects of their implementation. However, when there are not enough controls deemed feasible, the modeling demonstration is not possible. ${ }^{104}$ In this way, assurances to be provided by the modeling may be obviated by the lack of suitable controls.

The attainment demonstration modeling requirement and the feasibility constraint creates a coordination challenge for planners. Agency personnel somehow translate the will of the people - their tolerance for pesky rules and costly emissions controls through a filter of available technology to define what constitutes a feasible control measure. Models are then used to show that feasible controls are adequate to clean up the air, even though controls are chosen based on political will. In the next section, this coordination is found to reduce interest in model uncertainty too.

\section{All Feasible Measures}

The policy of implementing "all feasible measures" similarly obviates modeling for decision-making. Originating in the language of the CCAA, the state ozone standard must be achieved at a rate that is "reasonably practicable", which is defined as either reducing both $\mathrm{NO}_{\mathrm{x}}$ and VOCs by five percent annually, or implementing "all feasible measures” (CCAA 2001). DeMandel, BAAQMD, described how the state law translated into planning practice that obviated the modeling,

In the past, starting in the 90's, as an agency, the District made a decision that because of the laws that we're working under, the models really, at least for the State process, are not useful. The District decided that, in the 91 CAP, to get into an involved disagreement with ARB about what the model suggests was counter productive.

\footnotetext{
${ }^{104}$ This is what appears to be happening in the SJV for its 02 Ozone Plan (SJVUAPCD 2001).
} 
Some people strongly objected to that decision. So it was a controversial decision, at least among the people who had a technical orientation and felt that you should try to develop your plans based on the best technical information.

Under the State plan, we didn't have to. Initially, we did use the model. The model suggested that we couldn't find a path to attainment in any reasonable time frame. So the California Clean Air Act required us to implement "all feasible measures”. [T]hen you don't have much incentive to expend resources modeling.

\section{Effects of Models on the Planning Process}

Any single modeling study may have only marginal impact on the long history of a policy problem or a specific decision, but modeling as an on-going feature impacts the policy process. It influences the "the framework of the policy debate, the criteria for choice, the extent to which model results will be binding on the participants, and the limits of negotiation and compromise over policy outcomes” (Dutton, 1985, page 20).

In this study, models affected the process in three ways: (1) modeling was a central focus of the process itself, (2) consequently, one route of meaningful participation was via interfacing with the modeling, and (3) those who controlled the modeling had considerable influence on decision-making.

Starting with the LIRAQ model used by ABAG in the late 1970's, modeling grew to become a central feature of the planning process. By the 1990's, development of the SARMAP modeling system, and an observational database to support its application, was a key objective of San Joaquin Valley Air Quality Study.

SARMAP modeling results framed the conclusions used to support the 1994 SJV Ozone Attainment Demonstration Plan. Isopleths were prominent parts of Bay Area and SJV ozone plans. The SARMAP modeling system was also used to inform decisions to 
require selective catalytic $\mathrm{NO}_{\mathrm{x}}$ reduction technologies on Bay Area refinery and SJV petroleum production operations, and to assess pollution transport from the Bay Area to the SJV.

Given the focus on modeling, critique of planning required its engagement. Starting with LIRAQ, modeling capability or expertise became important for participating in air quality planning. CARB was forced to "get into the game."

Modeling results are the standard of scientific evidence and the currency of conversation. ${ }^{105}$ Arguments pertaining to control decisions are presented in terms of modeling output, often depicted in an isopleth. Both the BAAQMD and SJV ozone plans present isopleths to show how planned emissions reductions will yield air quality goals. Therefore, questioning modeling was a route to criticize plans.

Debate about SF refinery $\mathrm{NO}_{\mathrm{x}}$ controls centered on results of SARMAP and other modeling studies. Modeling was a prominent feature of dialogue between stakeholders. One way to be heard was to speak about the modeling.

Controlling the modeling meant considerable influence on the process because models provide the ammunition to defend decisions. Those in control of the modeling gained the upper hand in decision-making. Two specific decisions illustrate the roles of models and support this finding:

- requirements to implement $\mathrm{NO}_{\mathrm{x}}$ emissions reduction technology (selective catalytic reduction - SCR) on Bay Area refineries

- omission of the $\mathrm{NO}_{\mathrm{x}}$ SCR on steam generators in western Kern County.

\footnotetext{
105 Other evidence, such as observational data analysis, is an important part of decision-making deliberations too, but modeling is one of the main foci..
} 
In both cases, the SARMAP modeling system was used and discussed extensively during the debate preceding decisions. Modeling was the focus of debate. Those attempting to influence decisions did so by talking about modeling results.

In the case of the Bay Area refinery $\mathrm{NO}_{\mathrm{x}} \mathrm{SCR}$, industry, BAAQMD and CARB each used modeling to evaluate the efficacy of the controls. Modeling results consistently failed to provide incontrovertible support for $\mathrm{NO}_{\mathrm{x}}$ controls. Results indicated that ozone concentrations might increase in the Bay Area, while providing very limited air quality improvements downwind in the SJV. Furthermore, modeling indicated that more total controls of VOC emissions reductions might be necessary if the refinery $\mathrm{NO}_{\mathrm{x}}$ controls were installed. Nonetheless, CARB pursued a policy of implementing all feasible measures that included Bay Area refinery $\mathrm{NO}_{\mathrm{x}}$ controls. In so doing, CARB questioned the reliability of modeling results and cited broader policy concerns, such as high particulate matter concentrations, cost and technical feasibility.

By questioning selectively modeling results, CARB was able to pursue a predetermined policy. CARB de-emphasized the work of its own technical staff when their conclusions did not support refinery $\mathrm{NO}_{\mathrm{x}}$ emissions reductions in the Bay Area. CARB also questioned others' modeling selectively. Control of modeling, through either its production or endorsement, empowered CARB to highlight the results' uncertainties and to base decisions on other, more political considerations.

In the SJV, public agencies lacked some control over decisions because the regulated community had the right to define modeling scenarios. Sponsorship of the SJVAQS and Section 182(f) of the FCAA gave major point sources the right to request specific 
modeling simulations. By simulating the effects of $\mathrm{NO}_{\mathrm{x}}$ controls in western Kern County, the justification for controls was successfully questioned. The SJVUAPCD had little choice but to omit the controls from the 94 Ozone Plan. ${ }^{106}$ In this way respect, the regulated community exerted influence, via the SARMAP model, over the objections of the rightful decision-makers, the SJVUAPCD.

The example of omitting refinery $\mathrm{NO}_{\mathrm{x}}$ controls from the 94 Ozone Plan might lead one to conclude that the modeling was pivotal, not marginal. Further analysis reveals that omission from the 94 Ozone Plan did not prohibit controls. Controls were eventually required as part of “all feasible measures” needed to meet the state ozone standard, and to pursue the $\mathrm{PM}_{10}$ NAAQS (for example, see Jordan 1998). In the end, all that the regulated community gained was a delay, as the controls were eventually federalized in the $\mathrm{PM}_{10}$ Attainment Plan required per the FCAA (SJVUAPCD 1997, pages 4-7 and 715).

\section{Role of Uncertainty}

Now that the role of models is described, this section answers the questions: How is uncertainty used in air quality planning? Who uses or does not use it? Why or why not?

\section{Discourses of Uncertainty}

This section summarizes the narratives constructed using interview and archival data that were delineated, in part, according to discussion of uncertainties. It is evident that stakeholders use uncertainty opportunistically to support their policy narratives.

\footnotetext{
106 This is also an example of how meaningful public participation depended on more than just an ability to engage the modeling. It required being part of the research effort preceding planning decisions.
} 
Although this assertion is made later, it is a part of using uncertainty to fortify policy narratives.

\section{Feasibility and Control All Narratives}

All narratives allow for and give great consideration to uncertainty. Both the control all and feasibility narratives' recommended policy positions dependent on large uncertainty that cannot be reduced within regulatory timelines or, even, during the tenure of any one decision-maker. The feasibility narrative treats modeling as too uncertain to define reliably the shape of the ozone production possibility curve. Without reliable understanding of the curve, it is not possible to determine if $\mathrm{NO}_{\mathrm{x}}$ or VOCs should be controlled preferentially in an attempt to pursue the most efficient path toward ozone standards.

One respondent who chose not be cited directly referred to this approach as "micromanaging the path to attainment". In the statement, the respondent acknowledged those who seek a single precursor control strategy, but says the uncertainty is too significant to "micromanage" air quality. The feasibility narrative uses precautionary rationale to conclude that $\mathrm{NO}_{\mathrm{x}}$ emissions should be reduced along with VOC emissions reductions. The narrative justifies this position based on an inability to use modeling to implicate a single precursor and notes a broad set of air quality concerns that include particulate matter, visibility and environmental justice.

\section{Science/Efficiency Narrative}

The science/efficiency narrative acknowledges the significance of uncertainty and its irreducibility, especially within regulatory timelines. All of the narratives speak similar truths about these issues. 
The science/efficiency narratives’ time horizon, however, extends beyond election cycles or regulatory deadlines. It seeks decisions based on cost minimizing and equity criteria that are informed by available scientific understanding. It hopes to use models and other scientific evidence to define a control strategy that is the most cost-efficient path to attaining air quality goals.

In keeping with the science/efficiency narrative, the regulated communities called for more research, including modeling, to inform both the SJV and Bay Area $\mathrm{NO}_{\mathrm{x}}$ control debates. The agricultural and petroleum industries sponsored and helped to guide the SJVAQS. The petroleum industry used modeling by consultants, BAAQMD and CARB to examine the effects of Bay Area refinery and SJV steam generator $\mathrm{NO}_{\mathrm{x}}$ reductions. In the case of the Bay Area, WSPA offered to sponsor emissions reduction in the SJV in exchange for delayed implementation of Bay Area refinery $\mathrm{NO}_{\mathrm{x}}$ controls. The hope was that the delay would give the science time to convince agency decision-makers that Bay Area $\mathrm{NO}_{\mathrm{x}}$ controls were not needed.

When the petroleum industry forwards the science/efficiency narrative, there is emphasis on sharing the burden of controls amongst all sources of emissions. Industrial respondents lament ${ }^{107}$ that political will exists only to ratchet down emissions from point sources but not to exact stricter controls on other sources. The refining industry's willingness to fund agricultural water pump replacements in the SJV helped shift attention from petroleum to agricultural emissions sources. Indeed, agriculture is under increasing pressure to reduce its associated emissions (SJVUAPCD 2003).

\footnotetext{
${ }^{107}$ See quote of Ziman in Political Will discussion of Chapter IX.
} 
The regulated community highlights that lack of convincing scientific evidence that reducing $\mathrm{NO}_{\mathrm{x}}$ emissions in the Bay Area will lower ozone and particulate concentrations, or will improve visibility, either in the Bay Area or downwind in the SJV. This is a high standard given limited understanding of particulate formation processes, and the limited representativeness of the few ozone episodes modeled. Even with data analysis (Fairley 1996; Blanchard et al. 1999; Blanchard et al. 2000) and observation-based modeling (Blanchard 2000; Blanchard and Fairley 2001) to corroborate modeling, no uncontested and certain conclusions about these relationships are evident. There nonetheless should be some accountability, argues those forwarding the science/efficiency narrative. Furthermore, they say, when an emissions control strategy yields little benefit, as might be the case with Bay Area $\mathrm{NO}_{\mathrm{x}}$ controls, the agencies must admit the mistake (Ziman 2000).

Before spending money on $\mathrm{NO}_{\mathrm{x}}$ controls to reduce $\mathrm{PM}$ concentrations, the regulated community wants to know that the controls will be effective. This still unresolved question is a research objective of the CRPAQS. The NARSTO PM review summarized the state of knowledge for PM formation in the SJV, finding that VOCs reaction pathways may affect formation of nitric acid and, in turn, ammonium nitrate (NARSTO 2003). This suggests that VOC controls would be more effective at reducing particulate in the SJV. Like the ozone debate, there are calls for a focused reduction strategy based on understanding of the science. As CRPAQS is ongoing, the science/efficiency narrative suggests awaiting CRPAQS conclusions before implementing controls.

Without adequate scientific understanding, uncertainty remains significant. However, $\mathrm{NO}_{\mathrm{x}}$ is known to be a precursor to ammonium nitrate (Seinfeld and Pandis 1998). 
Observations collected as part of the CRPAQS indicate that nitrate can comprise a significant fraction of atmospheric PM concentrations in the SJV (Magliano 1998). Regional-scale visibility degradation is due to high concentrations of particulate matter. Understanding the relationship between $\mathrm{NO}_{\mathrm{x}}$ emissions, $\mathrm{PM}$ formation, and visibility is an additional layer of complexity layered atop burgeoning aerosol science. Given the uncertainty, CARB wants $\mathrm{NO}_{\mathrm{x}}$ reductions (i.e., feasibility narrative), whereas industry wants scientific justification first, per the science/efficiency narrative.

Regarding ozone, the science/efficiency narrative notes that a dual control strategy may place further demands on the search for feasible emissions. Under the right circumstances, which some believe exist in the Bay Area, a single-pollutant control strategy may necessitate fewer reductions of each pollutant than a dual control strategy. The isopleth used in the Bay Area's 01 Ozone Plan is shown again in Figure VIII-1. This time, the dual control strategy and the VOC-only strategy are shown. Indeed, the VOConly strategy provides a much shorter route to the $124 \mathrm{ppb}$ contour, thereby indicating fewer VOC controls would be needed to meet the standard if there were no $\mathrm{NO}_{\mathrm{x}}$ controls implemented simultaneously. The controls called for in the 01 Ozone Plan, and previous plans, indicate movement shown by the arrow labeled $\Delta$ SIP. Components of $\Delta$ SIP are $\Delta \mathrm{VOC}_{\text {dual }}$ and $\Delta \mathrm{NO}_{\text {xdual }}$. Pursuing a VOC-only strategy would require VOC controls equal to $\Delta \mathrm{VOC}_{\mathrm{VoC}-\text { only. }}$ Clearly, when the shape of the isopleth is as shown in Figure VIII- $1, \Delta \mathrm{VOC}_{\text {dual }}$ is greater than $\Delta \mathrm{VOC}_{\mathrm{VOC}-\text { only. }}$ Furthermore, because no $\mathrm{NO}_{\mathrm{x}}$ controls will be necessary for a VOC-focused strategy, it is appropriate to compare $\Delta \mathrm{VOC}_{\mathrm{VOC}-\text {-only }}$ with the sum of $\Delta \mathrm{VOC}_{\text {dual }}$ plus $\Delta \mathrm{NO}_{\mathrm{x} \text { dual. }}$. According to the science/efficiency narrative, 
then, by failing to implement a VOC-only strategy, society pays for unnecessary controls equal to the shaded area with dimensions $\Delta \mathrm{NO}_{\mathrm{x} \text { dual }} *\left(\Delta \mathrm{VOC}_{\text {dual }}-\Delta \mathrm{VOC}_{\mathrm{VOC}-\text { only }}\right)$.

The opportunistic use of uncertainties involves both highlighting and ignoring uncertainty. The argument forwarded by the science/efficiency narrative, as translated in Figure VIII-1, hinges on two vital assumptions that are not typically noted when discussing isopleths. ${ }^{108}$

The first assumption is that the isopleth is correct in its representation of ozone concentrations (i.e., ozone contours) as a function of emissions of $\mathrm{NO}_{\mathrm{x}}$ and VOC. As only one episode was modeled with SARMAP, the single episode is treated implicitly as representing myriad locations and meteorological conditions. ${ }^{109}$ The second key assumption is that the point of current condition is correctly located on the diagram, an assumption that hinges on the estimate of emissions.

\footnotetext{
${ }^{108}$ This is not to say that those using isopleths to illustrate their arguments are not aware of or honest about the uncertainties. They are aware of them, but still interpret some isopleths as indicating a single precursor strategy. This is the case for the Bay Area, but not for the SJV, where available isopleths (and corroborative) studies have not indicated clearly the need for a single-precursor control strategy (for examples, see Reynolds et al. 2002; Blanchard 2001).

${ }^{109}$ As noted above, however, the science/efficiency narrative uses data analysis and other corroborative studies to support arguments depicted using an ozone isopleth.
} 
Figure VIII-1: Bay Area Ozone Isopleth for Livermore

(Modified from BAAQMD 2001a)

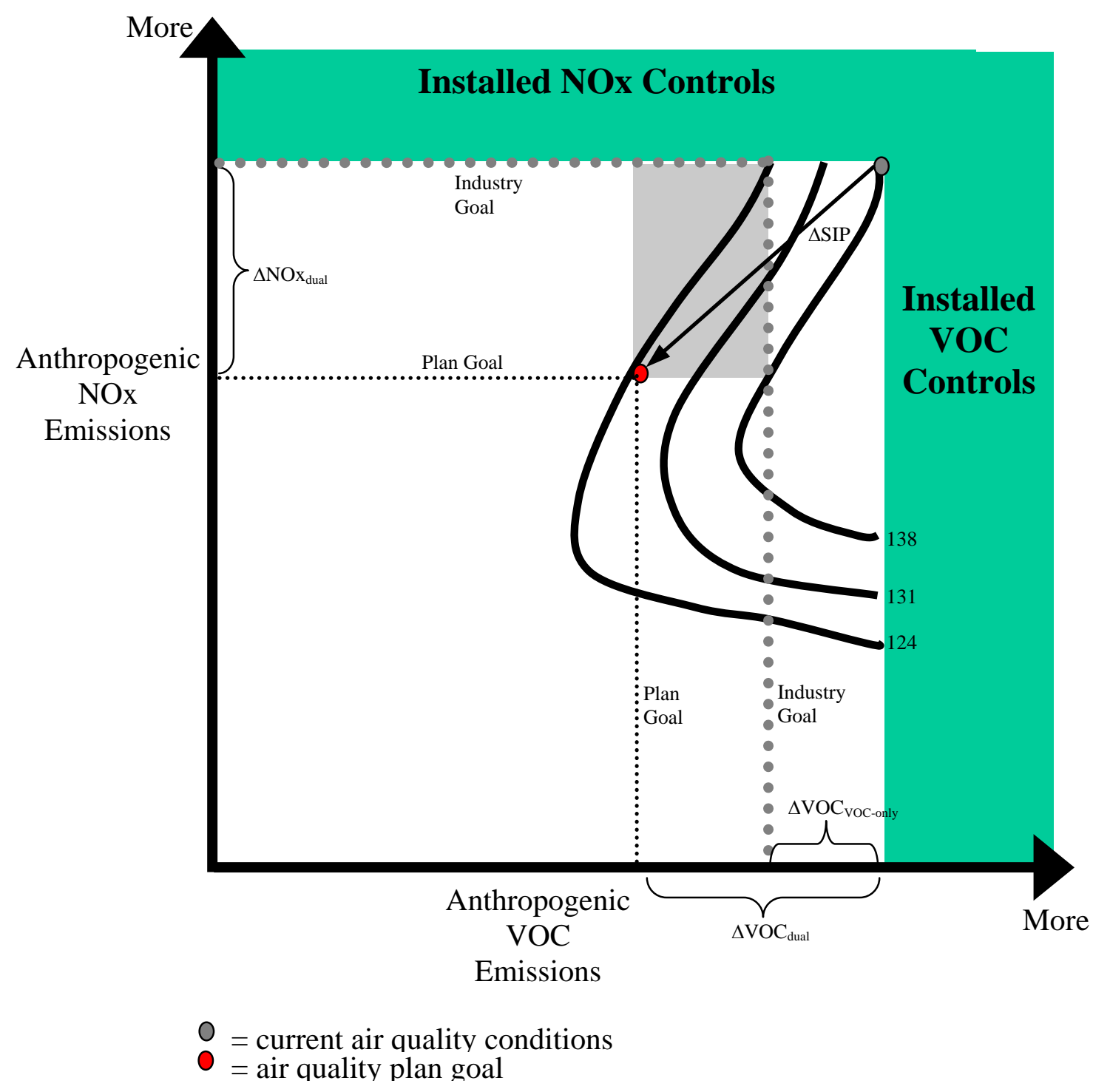


The importance of the first assumption about the accurate depiction of the shape of ozone contours is evident when examining a composite isopleth presented in the 94 Ozone Plan, shown here as Figure VIII-2. It shows that the isopleth implied neither a $\mathrm{NO}_{\mathrm{x}}$ nor VOCfocused strategy. There is no obvious ridgeline, nor obvious regions of $\mathrm{NO}_{\mathrm{x}}$ or $\mathrm{VOCs}$ limitation. The feasibility narrative uses the "flat" isopleth to argue for dual control, whereas the science/efficiency narrative seeks an emphasis on research to further resolve the isopleth and its uncertainties. Ideally, argues the science/efficiency narrative, modeling and other evidence will reveal a shorter path toward the $124 \mathrm{ppb}$ contour. The feasibility narrative believes any attempt to identify the shortest path to $124 \mathrm{ppb}$ is obscured by uncertainty and obviated by the need to get to the $90 \mathrm{ppb}$ contour, per the state standard, as well as other air quality and social justice concerns.

The second assumption about isopleths upon which narratives rely - that we actually know where we are in terms of emissions of $\mathrm{NO}_{\mathrm{x}}$ and VOC - is certainly incorrect. A brief exploration of the uncertainties attending emissions estimates reveals the tenuous ground upon which isopleth-based arguments rely. The isopleth used for the 94 Ozone Plan is reproduced as Figure VIII-3. This time, however, the whole emissions picture, so far as it is understood, is shown. Biogenic VOC emissions are included with anthropogenic emissions.

It is generally agreed that biogenic emissions are a major source of uncertainty associated with emissions estimates (Russell 1997; NARSTO 2000b). Using a survey of air quality experts, Hansen (2000) estimated the biogenic, mobile and other anthropogenic area source emissions for northeastern U.S. were uncertain by a factor of two. Ignoring that all VOC emissions are not the same, an estimate of biogenic 
emissions, as well as an error correction, is added to the isopleth in Figure VIII-3. The correction depicts the halving of the estimate of biogenic emissions in the 2002 update to the 94 Ozone Plan. ${ }^{110}$ That is, the current estimate of biogenic VOC emissions for the SARMAP modeling domain is half of the estimate used for modeling in $1994 .{ }^{111}$

Not depicted in Figure VIII-3 is the uncertainty associated with the anthropogenic $\mathrm{NO}_{\mathrm{x}}$ and VOC emissions estimates though they are known to be uncertain. Emissions estimates, and their uncertainties, are discussed at length in the Central California Air Quality chapter. Indicative of the uncertainty, the year 1999 emissions estimates of both $\mathrm{NO}_{\mathrm{x}}$ and VOCs increased by approximately one-third from the 94 Ozone Plan to Draft 02 Ozone Plan (SJVUAPCD 1994; SJVUAPCD 2001b). Independent research confirmed that the 1990 and 1999 emissions estimates used for the SARMAP modeling were quite uncertain (Marr 2002b).

The uncertainty due only to the biogenic VOC emissions estimate is sufficient to illustrate the potential to be in an entirely different regime regarding ozone production and precursor ratios. Although sensitivity to small changes in emissions might be accurately depicted in the isopleth, even with uncertain estimates of emissions, the large emissions changes being modeled (often 30 percent or more) pushed the model into ever more uncertain terrain. Accounting for these uncertainties undermines the science/efficiency narrative when it seeks a single precursor strategy based on a fairly

\footnotetext{
${ }^{110}$ Figure III-3 shows a correction related to biogenic VOC emissions. It says nothing of the impact that this correction will have on the shape of the isopleth, nor the impact that the correction has on predicted peak ozone and its sensitivity to changes in emissions. To explore these consequences, it is necessary to execute the model with a new emissions inventory.

${ }^{111}$ The estimate of biogenic VOC emissions is not reported in the 94 Ozone Plan. Nor is it published in the Draft 02 Ozone Plan, however, it does state that biogenic VOC accounted for as much as 80 percent of VOC emissions in the SARMAP modeling domain.
} 
precise location on the isopleth, even if the arguments are supported with other evidence. Although nobody has yet suggested a single precursor strategy be pursued in the SJV, this is the argument forwarded by the regulated community in the Bay Area. ${ }^{112}$

Figure VIII-2: SJV 1994 Composition Isopleth

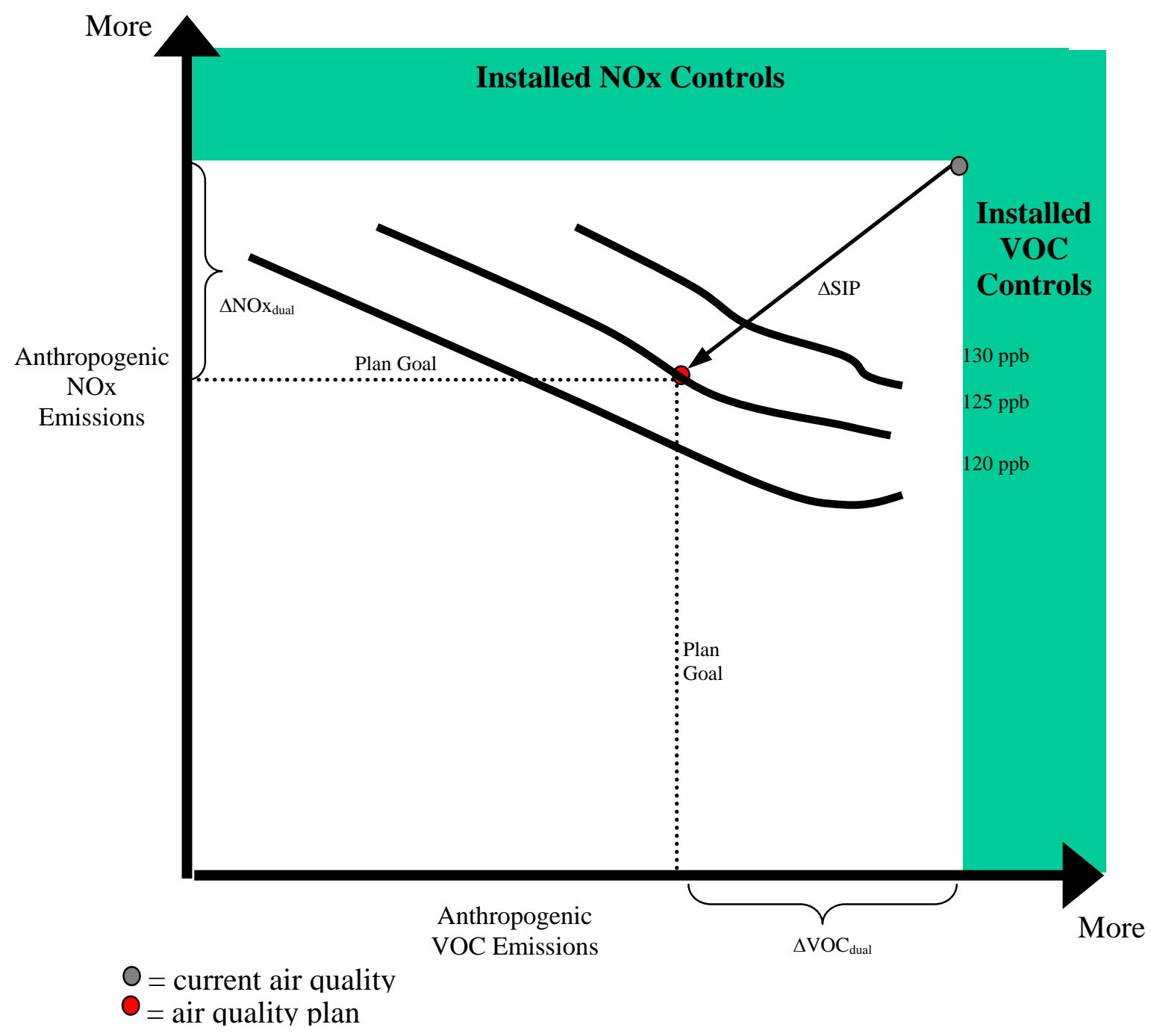

112 See quotes presented in Narratives discussion in Chapter IX. 
Figure VIII-3: Biogenic Emissions Uncertainty in the SJV 1994 Composite Isopleth

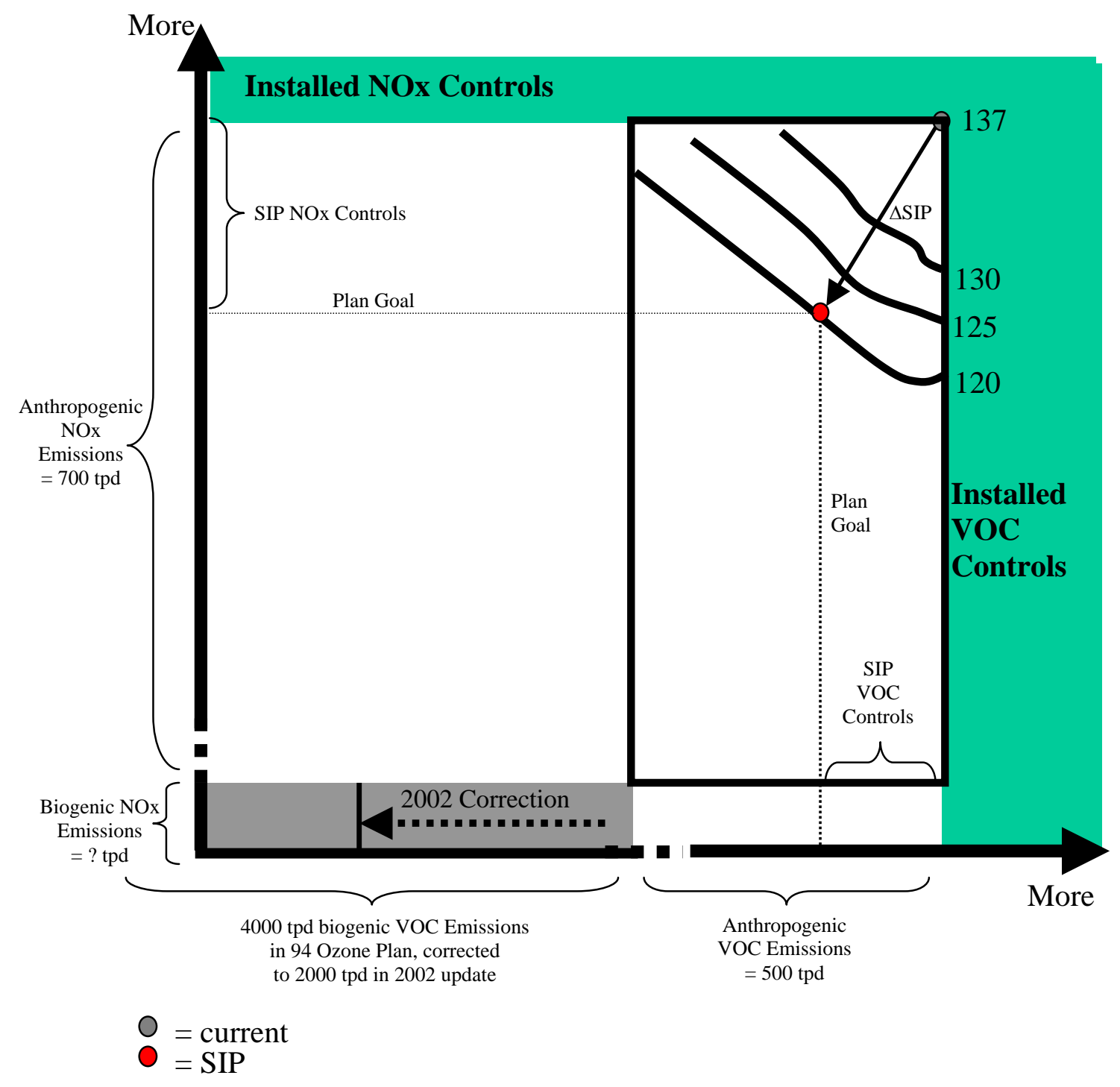




\section{Opportunistic Use of Uncertainty}

Stakeholder groups invoke concerns about uncertainty to support their policy positions. This behavior is evident in both policy narratives.

To the extent that all feasible emissions reductions are pursued, as is CARB policy, the uncertainties are used opportunistically for justification. CARB, air districts, and EPA use uncertainty advantageously and rhetorically to support the feasibility narrative. CARB used uncertainty as a reason to reject their own technical staff's work and to call for Bay Area refinery $\mathrm{NO}_{\mathrm{x}}$ controls. CARB did not, however, allow uncertainties to prohibit the use of SARMAP modeling as justification for the 1994 SJV SIP and their assessment of transport between the Bay Area and SJV. In this way, CARB considered selectively the uncertainties.

The science/efficiency narrative's version of the precautionary principle is to delay spending money on controls until they are shown to be effective and necessary. Without this information, it is possible that controls will be ineffective or, as may be the case for Bay Area $\mathrm{NO}_{\mathrm{x}}$ controls, counterproductive. In forwarding this narrative, the regulated community uses uncertainty to delay implementation of controls while awaiting studies to reduce scientific uncertainty. Given these behaviors, uncertainty may be both the bane and bounty of air quality planning.

\section{Assumptions About Uncertainty}

The logic that model uncertainty is irrelevant rests on two erroneous assumptions. First, that accounting for uncertainty would require additional controls to provide for a 
margin of error. Second, that the margin of error is not possible because control decisions are already constrained by the availability of feasible controls.

Many respondents believed that more knowledge of uncertainty will mean that more emissions controls will be necessary. Shipp, SJVUAPCD, provided an example of this thinking,

If the target is 30 percent [emissions reductions], and I know the error bar is big, which I think we do, then, if you're conservative, you say the target ought to be 40 or 50 percent. My personal view is that is true.

While it is true that planning for contingency controls is one way to address uncertainty, the respondents often did not recognize that the uncertainties may work both ways. As shown in Figure VIII-1, the Bay Area isopleth, prevailing evidence indicates that controlling both $\mathrm{NO}_{\mathrm{x}}$ and VOC emissions means that more total VOC emissions controls will be necessary. Thus, by failing to consider a single-precursor strategy, the problem of available controls may be exacerbated because more controls of both precursors may be needed.

\section{Communication and Management of Uncertainty}

Although there is discussion of uncertainty by stakeholders, explicit uncertainty information is not given to the public or transmitted up the decision-making hierarchy. Ozone attainment plans may note uncertainties associated with the technical bases of decisions, such as modeling and resulting isopleths, but the implications of uncertainties are not managed explicitly in the part of the plan presenting evidence that the required controls will be sufficient. Although corroborative evidence might be generated to support or bring into question the reliability of modeling results, this information gets 
decreasing attention as discussions move from technical to political realms. This raises two issues about the role of uncertainty in the planning process.

- There is a lack of clear and open discussion of the uncertainties.

- Rigorous assessment and management of the uncertainties is lacking in the decision-making context.

The preceding isopleth discussion is one example of incomplete disclosure of the uncertainties. A second example is provided by the 94 Ozone Plan. Respondents agreed that the plan presented two messages: (1) the plan is based on uncertain and evolving scientific understanding upon which the plan was based, and (2) the "attainment demonstration” using emissions estimates and modeling results that are not treated explicitly as uncertain.

In exploring the benefits of dual control versus a single pollutant focus, four scenarios were simulated and reported in the 94 Ozone Plan, as shown in Table VIII-B.

Table VIII-B: Reductions Scenarios Simulated in 94 Ozone Plan

\begin{tabular}{|l|l|l|}
\hline $\begin{array}{l}\text { Emissions Reduction } \\
\text { Scenario }\end{array}$ & $\begin{array}{l}\text { Domain-wide Peak } \\
\text { Ozone }\end{array}$ & SJVUAPCD Interpretation \\
\hline $50 \%$ of anthropogenic $\mathrm{NO}_{\mathrm{x}}$ & $>120 \mathrm{ppb}$ & $\begin{array}{l}\text { Attainment achieved in Kern and Fresno } \\
\text { Counties }\end{array}$ \\
\hline $\begin{array}{l}50 \% \text { of anthropogenic } \\
\text { VOCs }\end{array}$ & $130 \mathrm{ppb}$ & Nonattainment in Kern and Fresno Counties \\
\hline $\begin{array}{l}25 \% \text { of anthropogenic } \mathrm{NO}_{\mathrm{x}} \\
\text { and VOCs }\end{array}$ & $128 \mathrm{ppb}$ & $\begin{array}{l}\text { Attainment in Kern County, but not in } \\
\text { Fresno County }\end{array}$ \\
\hline $\begin{array}{l}50 \% \text { of anthropogenic } \mathrm{NO}_{\mathrm{x}} \\
\text { and VOCs }\end{array}$ & $115 \mathrm{ppb}$ & Attainment in Kern and Fresno Counties \\
\hline
\end{tabular}

These simulations led to a control strategy goal of reducing 40 percent of VOC and 38 percent of $\mathrm{NO}_{\mathrm{x}}$ emissions in the SJV. Alternatively, the simulates indicated that reducing 47 percent of anthropogenic $\mathrm{NO}_{\mathrm{x}}$ emissions with no further VOC emissions would also be sufficient. 
Why was a dual control strategy selected over a $\mathrm{NO}_{\mathrm{x}}$-focused strategy? What criteria were or should have been used to compare strategies? The science/efficiency narrative focuses on cost and efficiency, but the answers to these questions have other components, such as: Are the burdens of controls shared equity considered? What is the political will? Is it only politically tenable to exact controls on large point sources? Should other sources of emissions, such as agricultural diesel engines, cars, SUVs, BBQs, and lawnmowers also be reduced?

Although Table VIII-B summarizes a sincere exploration of one aspect of planning uncertainty, there was no attempt made in the 94 Ozone Plan to explain why one control path was chosen over another. Returning to Figure VIII-2, the SJV isopleth, it appears there are many dual control pathways that will achieve the 120 ppb contour with more or less the same reductions.

The control all narrative considers available evidence, such as isopleths, to be insufficient to define a perpendicular (i.e., shortest) control strategy for meeting the ozone standard. Instead, planners turn pragmatically to the list of available control measures, most of which are suggested by CARB and EPA. It was this list more than the efficient pursuit of ozone goals in light of modeling results that defined the control strategy.

\section{The Myth of Corroboration}

Further evidence of the lacking rigor in uncertainty assessment, and its misleading communication, is provided by the "weight of evidence" demonstrations used in ozone 
attainment plans. Arguments to pursue an emissions reductions goal do not rely solely on an isopleth. Other evidence was offered.

As discussed in Chapters VII and $X$, observational data and trends analyses are used in concert with modeling to demonstrate that the "weight of evidence" indicates the ozone NAAQS will be attained once planned controls are implemented. Some of these methods are less reliable than modeling, however. Simple "rollback" modeling does not depict complex, nonlinear chemistry, such as ozone quenching by NO emissions. ${ }^{113}$ Emissions trends analyses do not consider possible unanticipated changes in emissionsproducing behavior, such as the popularity of sport utility vehicles or growth rates that differ from past experience.

More reliable methods are sometimes employed too. As discussed in Chapter III PAQSM and Their Uncertainties, ambient measurements of $\mathrm{NO}_{\mathrm{x}}$ and VOCs may be compared to determine if observed ratios confirm those implied by the estimated position on the isopleth. Observation-based modeling is also used, whereby fundamental physical relationships are modeled using monitoring data. Although observations are subject to biases and may not be representative, these analyses do lend credence to "weight of evidence” conclusions. $^{114}$

Efforts to corroborate modeling results are indeed an important part of uncertainty assessment and management. Given the use of modeling as a tool to justify decisions,

\footnotetext{
${ }^{113}$ See Nonlinearity discussion in Chapter $X$ - Debates and Decisions.

${ }^{114}$ It is difficult to measure ambient concentrations of $\mathrm{NO}_{x}$; instead measurements usually represent $\mathrm{NO}_{y}$, which includes $\mathrm{NO}_{\mathrm{x}}$ plus products of $\mathrm{NO}_{\mathrm{x}}$ oxidation. $\mathrm{NO}_{\mathrm{y}}$ may consist of $\mathrm{HNO}_{3}, \mathrm{HONO}, \mathrm{N}_{2} \mathrm{O}_{5}, \mathrm{NO}_{3}$, peroxyacyl nitrates (PAN), organic nitrates, particulate nitrates, and any other reactive nitrogen compounds present (Seinfeld and Pandis 1998) Furthermore, VOC measurements are not typically located to capture ambient VOC emitted from biogenic sources; instead the measurements are located near human sources,
} 
however, corroborative evidence is presented to fortify the justification rather than to bring it into question. For example, the BAAQMD ozone attainment plans of 1999 and 2001 used a weight of evidence analysis to support reductions goals based on an isopleth that included trends and simple rollback analyses. Such unreliable supporting evidence, however, provided no additional confidence even though they were presented for this purpose. That these methods continue to be used despite that they are, in the words of the BAAQMD, "not considered a reliable quantitative analysis tool for ozone" is evidence of lack of communication and management of uncertainty.

\section{Model Performance Evaluation}

Lack of uncertainty communication does not mean uncertainty is not considered by decision-makers, even if that is the implication. Models used in the regulatory context must meet performance criteria. Performance is a hurdle to be cleared in the long process of ozone attainment planning. However, the criteria are insufficient to force stressful evaluation of performance (Roth et al. 1997; NARSTO 2000b; Fine et al. 2003). Furthermore, per the prior finding, once a model's performance is deemed adequate, planning proceeds without formal consideration of the uncertainties. Uncertainty information is not transmitted up the decision-making hierarchy in a complete manner. Instead, decision-makers must "internalize" concerns about uncertainty because available information about uncertainty, such as results of model performance evaluation, can not be applied directly to decision-making criteria, such as might be used in risk assessment and management (Stokey and Zeckhauser 1978; Morgan and Henrion 1990). 
Respondents concurred that performance criteria are insufficiently stringent or exhaustive. The resultant concern is twofold. First, a model that meets performance criteria may still be too uncertain to simulate reliably the change in air quality anticipated from emissions controls. This is a "signal-to-noise" problem, whereby the desired reduction in ozone (signal) is smaller than the model's uncertainty (noise).

The second concern about performance criteria is that they lack a requirement to identify offsetting errors that might make model performance appear better than reality. Without understanding of compensating errors, it is not possible to know if responses indicated by the model are likely to be observed in the real world. The model's responses to changes in emissions, for example, are used to generate the isopleths. This concern is exacerbated by the practice of "tuning" the model, whereby model inputs are adjusted (within their ranges of uncertainty) to improve model performance.

As documented in previous chapters, some believe that tenacious pursuit of uncertainty is rendered pointless by the other constraints on the planning process. These arguments are contradicted by apparent agreement that more information is preferred over less information. Despite her pessimism about the potential utility of model uncertainty information, Terry , CARB Executive Office, said,

More information is always good...[I]f someone can present us with a new way of looking at the information we have, it's always a good thing.

Terry and others who consider control decisions constrained by other factors deem uncertainty information obviated by these factors, notably “feasible” controls. However, in some cases, such as shown in Figure VIII-1 for the Bay Area, prevailing evidence indicates that the dual control strategy exacerbates concerns about the availability of 
controls because it means more VOC reductions will be needed. The science/efficiency narrative is hyper aware of this possibility; the feasibility narrative dismisses it based on other air quality concerns. This debate is a key reason to consider the uncertainties explicitly and the pleas of the science/efficiency narrative are quite persuasive in this respect.

For example, consider the observation that emissions estimates for both the Bay Area and the SJV are quite uncertain. The feasibility narrative considers this uncertainty adequate reason not to try to "micromanage” ozone using single-precursor emissions control strategies. The science/efficiency narrative, however, suggests it is possible to conduct sensitivity and corroborative studies to determine if uncertainties in emission estimates have bearing on the conclusion that $\mathrm{NO}_{\mathrm{x}}$ controls in the Bay Area will be counterproductive.

Executive decision-makers do not seek detailed information about the uncertainties. Instead, they assume technicians do what they can to deal with the uncertainties, discuss uncertainties informally with technicians, and then "internalize” their understanding when making decisions. They know that performance criteria are insufficient, but trust modeling technicians to do their best, within time constraints, to improve model performance. The result is a misalignment between decision authority and understanding of the uncertainty.

This is not to say that modelers do not continue to explore uncertainties and to improve modeling studies. They do. CARB should be commended for their continued testing of SARMAP after the 94 Ozone Plan was approved by EPA. The research 
process, including model and emissions inventory improvement, is ongoing. However, there is little indication that the uncertainties are considered formally or rigorously when planning decisions are being made.

Respondents indicated that public agencies are indeed moving towards incorporating uncertainty information into decision justification. Terry said, “...we have to deal with uncertainty one way or another, either technically or politically.” There is a demonstrated savvy in discussing uncertainty, one developed over time with experience. But with an inverted relationship between decision-making authority and understanding of the uncertainties, as depicted in Figure VIII-4, the means of incorporating uncertainty is far from obvious. In addition to decision-making constraints, there are two significant barriers toward using uncertainty information in decision-making: its production and communication. Technicians must produce it and then it must be communicated, clearly, clearly and completely, up the chain of decision-makers. Findings from this case indicate that there is little impetus to commit the resources necessary to overcome these two barriers. 


\section{RECOMMENDATIONS}

\section{A. Introduction}

Using California's Central Valley as a case, this study has shown that modeling is used as part of a political process rather than as a bastion of objectivity. Uncertainty is utilized for political ends. Furthermore, an important route of participation in air quality control decisions is via the modeling, so those who lack modeling expertise do not participate meaningfully. This chapter presents two sets of recommendations toward the three goals of consensus, inclusion, and rational decision-making using uncertainty information.

The first set of recommendations intends to augment modeling products through the evaluation, estimation and communication of uncertainties. Consensual and participatory aspects of process are addressed in a second set of recommendations. Last, recommendations address the broader, post-normal, social aspects of process.

Managing uncertainty in air quality planning requires consideration of several types of uncertainties. Suggestions here go beyond the quantification of uncertainties in modeling, even if that is a place to start. The need for transparency, disinterested review, and documentation is motivated by social concerns encompassing the technical effort. Uncertainty in this context is more than what goes into the models, such as input data and mathematical formulas. Uncertainties are also associated with how the modeling is conducted, such as the choice of what to simulate. Encompassing the modeling task is uncertainty about how modeling results are interpreted and weighed against other evidence. Last, there are relevant uncertainties exogenous to the problem at hand, such 
as changes to the ozone standard and problem definition that extends beyond ozone to, for example, include ambient particulate matter, visibility degradation and environmental injustice.

\section{B. Toward Rationality}

Thirty years ago, Brewer (1973b) warned of the challenges of considering uncertainty in model-based planning:

Conscious consideration of uncertainty increases the inherent complexity of the theoretical context and the analytical difficulty of the formalized representation of that context. Uncertainty about the reality context remains; deciding if and how to draw uncertainty into replicates of that reality is at issue. To the extent that one supersedes determinism to account for errors of measurement and specification and to consider stochastic processes, a system becomes larger, more complex, and analytically more difficult.

Brewer's concern notwithstanding, calls for treating uncertainties are ubiquitous but few steps have been taken to actually address it explicitly. Management of modeling uncertainties in decision-making requires their identification, evaluation (with quantification, if possible), communication and translation to decision alternative.

Applied to air quality planning, risk assessment and management has three steps: (1) begin with a qualitative assessment of the likelihood that a modeling study will be reliable, (2) proceed with model uncertainty evaluation with the goal of quantifying the probabilities of alternative outcomes, and (3) make decisions using a risk management paradigm.

\section{Qualitative Assessment}

The sources of uncertainties in air quality modeling are well documented (Fine et al. 2003; Russell 1997; NARSTO 2000b; Russell and Dennis 2000; Seinfeld and Pandis 
1998). Evaluating uncertainties in any individual modeling application often requires custom investigations (for example, see Roth et al. 1998). I recommend the qualitative approach suggested by Roth (1999) because it is useful for determining if a selected modeling application is likely to yield reliable results. Roth suggests twenty questions be asked to identify the need to modify an application before proceeding with regulatory decision-making. Questions pertain to the following topics:

- Modeling Application Planning

- Are the specifications of the model application consistent with sound practice, and are problems observed in past applications dealt with adequately?

- Is a comprehensive and rigorous modeling protocol prepared?

- Data Needs

- Is there available a database for surface air quality data that is suitable for comparing observed and modeled concentrations of ozone, $\mathrm{NO}_{\mathrm{x}}$ and VOCs?

- Are transported pollutants likely to be significant in the modeled airshed? If so, is there an adequate database or reliable output from a model of coarser spatial scale suitable for specifying boundary conditions?

- Are sufficient meteorological data available at the surface and aloft to support modeling and reliable representation of meteorological fields?

- Are sufficient aloft air quality data available for comparing observed and modeled concentrations of ozone, $\mathrm{NO}_{\mathrm{x}}$, VOCs and other chemical species?

- Evaluating Meteorological and Emissions Models

- Does available evidence indicate that anthropogenic emissions, by source category, are represented adequately with appropriate spatial and temporal scale and with little or no bias?

- Are ozone estimates likely to be sensitive to biogenic emissions? If so, does available evidence indicate that biogenic emissions are represented adequately?

- Has there been adequate effort to evaluate the quality of representation of meteorological fields?

- Evaluating the Air Quality Model 
- Is the performance of the chosen PAQSM at least as good as alternative formulations and does it represent the state-of-the-science in depicting significant, dynamic processes and large stationary source emissions plumes? Is the numerical solution method the most accurate and suitable, and are associated errors demonstrated to not pose a problem? Are chosen horizontal and vertical resolutions appropriate?

- Has performance evaluation been conducted with sufficient rigor? Is the quality of performance acceptable given the decision-making needs?

- Has a sufficient diversity of meteorological and transport conditions been studied?

- Has the model been evaluated for one or more episodes with only presimulation interventions that are standard practice or are identified in the first one or two simulated episodes?

- Has an effort been made (a) to identify, a priori, potential compensating errors and to design tests for detecting them, (b) to assess the accuracy of representation of model components a posteriori, or (c) to evaluate potential compensating errors through other means?

- Other Questions

- Has sensitivity analysis been used methodically to assess the potential presence of compensating errors?

- Have the findings of modeling been corroborated through other, independent means?

- Has an attempt been made to estimate uncertainties associated with modeled ambient concentrations, for both the base case and future cases?

- Have the quality of emissions projections and their uncertainty ranges been evaluated and estimated using other available information?

- Has adequate documentation of the modeling application and evaluation been prepared?

- Has an independent, in-depth peer review of the model application and evaluation been conducted, and was the effort judged acceptable?

\section{Quantitative Assessment}

Probabilistic statements are the preferred means for describing uncertainty (Stokey and Zeckhauser 1978; Morgan and Henrion 1990; Casman, Morgan et al. 1999). Such 
statements allow decision-makers, for example, to answer questions like, "What is the probability of attaining air quality goals when models predict so?”

Methods for evaluating uncertainties in PAQSM are detailed in Chapter III - PAQSM and Their Uncertainties. Sensitivity studies can be used to provide information about the relative importance of model parameters and their uncertainties; information that helps planners to set research priorities. Simulations using sets of inputs from within their ranges of uncertainty can be used to quantify (a) how outputs are sensitive to input uncertainty, and (b) when accounting for inputs' uncertainties iteratively and repetitively, output uncertainty due to inputs’ uncertainties.

Diagnostic and corroborative analysis also could be used to characterize model accuracy. Diagnostic tests assure that components of the modeling system are performing accurately. Corroborative analyses may involve model-to-model comparisons or consideration of other information, such as observational data. Suggested as a complement to emissions-driven models, observation-based methods (OBMs) rely on monitoring data instead of uncertain emissions estimates. One approach to OBMs is to use simpler models, such as chemical kinetic equilibrium relationships, to estimate the relative advantages of control options. ${ }^{115}$

Another way to estimate uncertainties is to query experts using the Delphi Method (Helmer 1963; Dalkey 1968) or some derivative. For example, Hanna et al. (2001) elicited experts’ opinions about air quality modeling conducted to address a major

\footnotetext{
${ }^{115}$ For example, the EPA accepts the use of the Measurement-based Analysis of Preferences in Planned Emission Reductions Ozone (MAPPER) model to evaluate the relative efficacy of $\mathrm{NO}_{\mathrm{x}}$ or hydrocarbon controls. Mapper relies on a simple reaction rate model and uses as input local observations of pollutant concentrations. (See: http://www.epa.gov/oar/oaqps/pams/analysis/noy/noxtxt.html\#observational).
} 
regulatory issue: controls needed to attain the NAAQS for ozone in the eastern United States.

Ultimately, these disparate methods need to coalesce into a statement about outcome probabilities. That is, for each possible outcome, modeling uncertainties are aggregated into an estimate of forecasted likelihood that outcomes, both desired and otherwise, will be realized. Though many methods are available to generate uncertainty estimates, no syntheses have been yet developed for air quality modeling applications (Fine et al. 2003).

In addition to evaluation and synthesis, methods are needed to communicate information about uncertainties. One potentially productive research avenue is to develop a means of presenting isopleths with uncertainty. As the products of modeling, isopleths are commonly integrated into planning as heuristic decision tools. Though some isopleths are limited by their representations of locations, meteorological conditions, and the ozone forming potential of VOCs species, it may yet be possible to modify the isopleth to depict the decision space shown in Table IX-A to include uncertainty information. The isopleth might be modified to map the probability of being located in different areas of the decision space, which correspond to the four quadrants of matrix in Table IX-A. Other approaches to presenting uncertainty information are possible, such as bounding analyses that help limit the range of possible choices (Morgan 2001; Casman, Morgan et al. 1999). 


\section{Risk Management}

In risk management, decision alternatives are compared based on outcomes' desirability and probability. When both characteristics are well understood for possible outcomes, the decision-maker has complete information about the risks associated with each alternative and their relative attractiveness. I recommend this approach for air quality planning by using modeling and supporting analyses to calculate the probabilities, and inclusive, consensus-based public discussion described below to estimate desirability.

Through public discussion, alternatives should be compared using their estimated probability of success, as well as other criteria that describe desirability. A lot has been said about using models to describe the probability of meeting goals once a proposed reductions strategy is implemented. ${ }^{116}$ This is just one element of risk; other relevant risk criteria address technical and economic feasibility, environmental justice, sustainability, and equity. The latter criteria consider not only what is at risk, but who risks it. ${ }^{117}$ Yet another component of risk pertains to time, since uncertainty increases with the timeline of projections (Casman et al. 1999; Ascher 1976).

Two key findings of this research are that emissions reductions alternatives are constrained by available "feasible" measures and that many assume consideration of uncertainty will necessitate more, not less, emissions reductions. Combined, these findings indicate that uncertainty information is obviated the "feasibility" constraint. To address this issue in a transparent and democratic manner, public debate should focus on

\footnotetext{
${ }^{116}$ The resultant uncertainty information can be used in the research process, too. These uses are listed in Chapter III - PAQSM and Their Uncertainties.

${ }^{117}$ For example, power producers risk the costs of emissions control that may not contribute to cleaner air,
} 
the definition of what constitutes a feasible emissions control technology (or policy). The definition should endeavor to incorporate all stakeholder costs in addition to those incurred by the emissions sources, such as adverse health impacts. For example, those engaged in discussions need the opportunity to understand how a decision to forego emissions reductions (or, to define feasibility restrictively) may translate into health effects and other social costs. Similarly, the costs of implementing controls need to be explained clearly so that public advocates will, for example, understand how those costs are pass-through from producers (i.e., emissions sources) to consumers (e.g., public citizens).

\section{Toward Consensus and Inclusion}

Sarewitz et al. (2000) say the scientific project of using computers to predict earth systems behavior "exists in a social and political milieu" of a "prediction enterprise" that is both product and process. The process involves three non-sequential parts - research, communication and use - that when working well increase the chance of good decisions. Sarewitz et al. (2000) call for a change in focus from prediction as a product to the prediction process. ${ }^{118}$ Two recommendations for process changes to make decisions truly consensus-based are (1) to include all interested stakeholders and (2) to give them a legitimate opportunity to participate meaningfully. Doing so will forward the goal of "high-quality science-based environmental decisions."119

\footnotetext{
whereas the exposed public risks adverse health effects of air quality degraded by emissions.

${ }^{118}$ Brewer (1973, page 293) suggested that rather than expecting a modeling exercise to produce "the" answer, "A more productive strategy might be to devise questions, techniques, and procedures that shift the focus of attention away from particular solutions and over to the difficulty of the problem itself."

${ }^{119}$ The EPA Science Advisor Board (SAB 2001) describes "high-quality science-based environmental decisions" as:
}

- Based on careful and complete review of critically evaluated scientific evidence. 


\section{Consensus}

Given high decision stakes and different opinions about the problem, with risks shared unevenly and understood incompletely, I recommend a decision-making approach that pursues legitimate consensus by giving all parties a real opportunity to participate in decision-making. Findings from this research concur with the literature (Fischer 1990; Funtowicz and Ravetz 1990; French 1993; Yearley 1996; SAB 2001) that science-led decision-making creates barriers to participation by the lay public. Consequently, several parties gain differential access through expertise. Notable in Central California was the extensive participation by representatives of the petroleum and agricultural industries in both research and planning to improve air quality.

In the case, CARB's policy of implementing all feasible emissions controls is a partisan position representing environmentalist values. Without representation from poor communities and environmental advocates, agencies speak on their behalf. This helps to explain why CARB's policy position is resistant to change, and CARB's willingness to use scientific evidence selectively. If all parties have opportunities to voice their opinions, then CARB and other public agents will have more freedom to be centrist, objective facilitators. They would have less need to provide proxy for opinions that, though legitimate, are not otherwise represented.

When discussing the incorporation of the lay public, concerns are often raised about the prospects of their educated input. If the science is so complex that it confounds scientists and policy analysts, how can the public be expected to understand? The Air

- Based on analyses well-established by decision and policy scientists (e.g., risk management)

- A combination of scientific understanding and insights with appropriate value judgments that reflect public preferences and EPA's responsibilities. 
Districts and politicians claim to know the political will, including the costs and inconveniences that will be tolerated in pursuit of clean air. Many AQMD board members whose job it is to review and approve air quality plans are elected officials. As politicians, they purport to know and represent the interests of their constituents. However, in practice, there is little opportunity for board members to influence plans. Agency staff encourage speedy plan approval when deadlines are looming (or passed) and there are grave consequences for impeding progress. Consequently, air quality plans are not assured of incorporating public opinion either directly or by proxy.

The case revealed that for several reasons the decision-makers representing the public interest did not manage uncertainty in planning decisions explicitly or adequately. Information about the uncertainties associated with technical evidence, such as modeling results, was not communicated up the chain of authority from modelers to planners to executive decision-makers. Attempts to "internalize" decisions led to conflicts between personal world-views and available scientific understanding (Landy et al. 1990), and were also overwhelmed by other concerns, such the availability of feasible emissions measures. There were also incorrect assumptions about the implications of uncertainty; that it meant more emissions reductions would be needed and was therefore obviated by other constraints.

One solution to these concerns is to include the lay public directly. Here, the "lay public" means environmental and poor community advocates ${ }^{120}$. Inclusion entails involving these advocates at planning meetings and on committees early in processes,

\footnotetext{
${ }^{120}$ Business interests are part of the public too and deserve representation. Though small business without industry associations may have difficulty engaging the process, major emissions sources, such as the
} 
and relies on review, approval and translation of technical evidence by disinterested experts.

To apply these recommendations to the SJV, the Policy and Technical Committees need environmental and community representatives who are provided with expertise advisors. Typically, doctors, lawyers, and leaders of religious, civic and residential groups represent the public living in poor, disadvantaged or severely polluted communities. In this work, those representatives said they would reject invitations to join the Policy and Technical Committees. Indeed, human and financial resource limitations impose severe constraints on these representatives (SAB 2001). Furthermore, joining the discussion, with or without a real opportunity to exert influence, implies endorsement. Thus, there is a dual, reinforcing problem of technocratic processes that impede understanding and participation by all stakeholders, and these same stakeholders who do not want to join a process pre-defined to limit their opportunities to influence decisions. Nonetheless, they have good reasons to engage in the discussion and need only to believe doing so is worth expending their scarce resources and risking their reputation. For them to be coaxed into the process, consensus-based decisions offer more hope than litigation and protest. The next section recommends incorporating stakeholders early and continually in the process, giving them opportunity to make as well as critique decisions, and to provide expert advisors for the lay stakeholder groups previously not involved and now skeptical of agency-produced, industry-funded research, such as modeling. 


\section{Inclusion}

For legitimate consensus-building, participant involvement must be empowered, rather than token (Arnstein 1969). Brewer (1973b) wrote that social ethicists should analyze value assumptions and that what is at issue is "how they to gain access and, once given access, how they might comprehend the content of the assumptions.” In this research, the environmentalists and community activists should be considered the social ethicists who do not currently have access. I offer two recommendations to give these groups opportunities to engage the process, including understanding the risks.

First, involve all stakeholders early in the process. ${ }^{121}$ Such involvement will include helping to define modeling studies and to determine how those studies will be used to inform decision options (e.g., generating information about the probabilities of costs and benefits associated with alternative control strategies). Two additional examples of meaningful involvement are (1) helping to generate or, at least, having an opportunity to critique, estimates of future conditions, such as land use changes, growth rates and associated emissions, and (2) selecting emissions controls scenarios. This latter task will provide juxtaposition for major stationary sources that are given the opportunity, though Section 182(f) of the CAA, to execute modeling simulations to determine if their individual emissions need be controlled to meet ozone goals. In the case study, a modeling simulation was used by petroleum interests to successfully challenge the SJVUAPCD’s plan to require $\mathrm{NO}_{\mathrm{x}}$ controls on major sources in western Kern County.

\footnotetext{
${ }^{121}$ Citing Wynne (Wynne 1988), Epstein (Epstein 1996) wrote the only way to open up the process is to "expose the uncertainty and value choices, and then convince people of the considerable importance of participating in such research even after they understand just how messy it truly is and how bounded is the usability of the knowledge produced by it.”
} 
The second recommendation for involving stakeholder group in a legitimate manner is to provide them with expert advice. Using the Comprehensive Environmental Response, Compensation, and Liability Act (CERCLA 1980) as a guide, provide “reasonable opportunity for public comment on any proposed plan” and

"grants to any group of individuals which may be affected...to obtain technical assistance in interpreting information with regard to the nature of the hazard, remedial investigation and feasibility study, record of decision, remedial design, selection and construction of remedial action, operation and maintenance, or removal action” (§ 9617(e))

There are two practical approaches to expert assistance. Experts may be contracted to serve on behalf of interest groups. Alternatively, it may be more desirable to facilitate and augment stakeholder participation by disinterested, expert peer review (SAB 2001). This latter option is useful if it generates information using a set of rules for the communicative use of technical information that builds trust between lay stakeholders, experts and technical evidence (Innes 1998).

Expert peer review is a method for quantifying uncertainty, but it might also be used to approve or label the products of research ${ }^{122}$. "Science courts" were proposed by Kantrowitz in 1967 (Majone 1989). Demerjian et al. (1995) propose using panels of experts to evaluate the adequacy of modeling studies used in air quality planning.

\footnotetext{
${ }^{122}$ Funtowicz and Ravetz (Funtowicz and Ravetz 1990) defined the NUSAP approach, whereby data described using five characteristics. Numerics, Units and Spread indicate error or imprecision. A qualitative Assessment is made to capture experience with "deeper uncertainties that not in the 'error bar' of the given experiment” (Ravetz 1999). Last is a Pedigree, which may take the form of a matrix, to express the data's history and intended function. For more description of NUSAP, refer to Van der Sluijs (2002a). For an example of NUSAP applied to estimating VOC emissions from paint, see Van der Sluijs et al. (2002b).
} 
The qualitative approach of Roth $(1999,1058)$ incorporates “independent, in-depth peer review of the model evaluation and application”. Brewer (1973b) calls for the institution of expert modeler reviews, and the development of performance evaluation protocols. In PAQSM, both have been tried. The expert review takes the form of a technical committee supporting a specific modeling study. Performance evaluation protocols developed by EPA and CARB are generally applied, but are acknowledge as incomplete by modelers and planners alike.

To assure quality science in large environmental assessment programs, (Winstanley et al. 1998) propose the use of a "governing board comprised of a consortium of stakeholders", as well as external peer review, strong and flexible program management, and a "semi-permeable barrier" between scientists and policy-makers. To facilitate permeability, diligent electronic record-keeping will allow others to reproduce or, at least, review the work of modelers. In the Central California case, Jim Sweet , a modeler at the SJVUAPCD, said "despite the effort to document, there is never enough detail.” Brewer (1973b, page 238) noted that documentation can never be “overly rigorous” and suggested as a rule of thumb that "as much money should be spent on documentation as on the model itself."

Akin to expert peer review, lay stakeholders might be gathered to form a "values jury” to suggest courses of action (Brown et al. 1995). Brewer (1973a) similarly suggests a "decision seminar" to "translate a decision-making context into terms that make it understandable and that suggest ameliorative action”. 
The SJVAQS Technical Committee was like a science court. The petroleum and agricultural industries were rightly represented, as were power producers, and local, county, state and federal officials. Even the military had a seat, but not the environmentalists and the poor. In the case of Central California air quality planning and research, the CERLCLA language means providing environmental and community advocates with expert representation on the Technical and Policy Committees overseeing air quality studies, as well as help in critiquing air quality plans.

\section{Modeling for Post-normal Problem Solving}

Air quality modeling is an example of regulatory post-normal science because it has large system uncertainties, values in conflict, high decision stakes and short timelines for decision-making (Ravetz 1999; Jasanoff 1990; Jasanoff 1996b). Consequently, the problems identified in this research "cannot be solved within the confines of the computer system” (Ravetz 1999, page 278). Hunt and Shackley (1996) wrote:

Although analyses from the sociology of scientific knowledge have largely undermined the belief in science as a form of pure knowledge with direct access to the real world, these studies have yet to be translated into the real world of science and policy.

NARSTO (2000a) and Demerjian et al. (1995) identify the limitations of current practice applying PAQSM for decision-making, and both offer many useful improvements that focus on using technical information to facilitate decisions. Yet, the social challenges remain unheeded in these scientific assessments. Several processrelated prescriptions are available. The policy sciences and planning literatures provide deeper understanding of the modeling task, as well as suggestions to complement technical improvements and uncertainty analyses for PAQSM. 
Models can be used to facilitate, not obstruct, the process but it will require modifying current practice. Several scholars offer rules for the using modeling results to inform decisions (Brewer 1973b; Winstanley et al. 1998; Ascher 1976; Innes 1998; Korfmacher 1998; Jager 1998; Morgan and Henrion 1990; Sarewitz et al. 2000):

1. Use models for directional guidance, not for prediction.

2. Use the simplest model that is still appropriate, but no simpler. Clarify the tradeoffs of selecting one modeling system over another.

3. Be clear about assumptions and the results' sensitivity to them; update assumptions regularly.

4. Acknowledge and communicate uncertainties completely. Document extensively and clearly. Allow independent replication for verification. Establish a priori criteria for model performance that include the identification and reduction of bias. Test all claims, assumptions and constraints for their factual basis, clarity, and comprehensiveness.

5. Facilitate interaction and understanding between the modelers and policymakers, and between the regulatory staff and the interested public; pursue pluralism and consensus during all phases of research and planning

6. Give all participants an opportunity to participate meaningfully. Participants must present credentials; required credentials must be based on more than an ability to pay. Assist lay stakeholders participate by helping them to understand and engage technical evidence. Identify, contract, listen, and respond to peer reviewers.

7. Acknowledge who is in control of the modeling and provide for disinterested oversight in the form of an expert review panel.

Dutton and Kraemer (1985) examine who controls the modeling and whose interests are served by modeling. Using these two dimensions on a matrix (see Figure VI-3), the Central California case revealed aspects of technocratic and consensual processes, but was used for partisan purposes too. Recommending comprehensive uncertainty assessment and risk management moves the process toward rationality. Efforts in pursuit 
of representation yield a more consensus-based approach. Together, these changes create processes reliant on technical experts (for uncertainty information) to serve pluralistic, non-partisan interests.

As Ozawa (1991; 1996) explained, science can be a mechanism of accountability when an independent entity makes decisions on purely scientific grounds. Yet, science and uncertainty may be used as tools of persuasion too. Points by Greenberger et al. (1976) that the political setting determines how model results are received are confirmed in this work The political setting influenced agencies' ability and willingness to rely on SARMAP results when debating requirements for $\mathrm{NO}_{\mathrm{x}}$ controls on Bay Area refineries. Ultimately, modeling results were overwhelmed by politics and a priori policy agendas. Neither the BAAQMD nor CARB could pass the "red face" test by allowing refineries to avoid $\mathrm{NO}_{\mathrm{x}}$ controls even though modeling suggested the reductions would cause local disbenefits and very little downwind air quality improvement.

Uncertainty is similarly wrapped up in the politics. Those who perceive uncertainty information as threatening their agenda will likely resist it. Such resistance is problematic when originating with those in control of the modeling, as was the case in Central California. Returning to Brewer (1973b), modeling was used in this case to shield, persuade, validate, question and slow down decisions. Thus, uncertainty information was sought for its strategic, as well as informative, purposes. When used for discovery (e.g., descriptive clarification), uncertainty information is relevant for evaluating the veracity of findings and for setting research priorities. Air Districts are charged with applying models for accountability, validation, and description. This case showed models also used for persuasion and uncertainty information was viewed at times 
as potentially undermining agency power, especially with respect to the modeled "attainment demonstration".

\section{Attainment Demonstration}

The "attainment demonstration" requirement is cited as one reason for the lack of explicit management of modeling uncertainties in plans. Planners need the flexibility to admit that the models are used directionally and that attainment might not occur as predicted by the models. Using models for directional understanding, rather than for prediction, will only be allowed if the attainment demonstration language of the FCAA is modified or interpreted differently. EPA must be willing to accept regulatory decisions using admittedly uncertain models. That is, currently the "attainment demonstration" is black or white - either the model indicates attainment or it does not - when in reality there is uncertainty. Grey areas need to be acknowledged and pursued, not painted as black and white. Once planners believe they have legal flexibility to admit to uncertainty, they can then proceed with risk management decisions.

\section{Periodic, Iterative, Adaptive Review}

Long since proposed (Holling 1978; Walters and Holling 1990) and recently embraced (Lee 1993), adaptive management is a way to manage uncertainties due to incomplete knowledge and inexperience that relies on feedback from experimental results. Adaptation is akin to Bayesian methods that, in this context, translate new information acquired through "experiment" and experience with emissions controls to a revised risk assessment (Abbaspour et al. 1999). For air quality, this means measuring actual emissions and air quality to determine if reductions are being achieve and having desired effects (Demerjian et al. 1995). Although air quality plans contain contingency 
measures, they have proven to be insufficient. Again, as shown in the case, this is due to insufficient controls deemed feasible.

Currently, planning cycles are defined by laws, laws’ interpretation by EPA, or court rulings. Though the CAA calls for plan updates at regular intervals, the slow legal and administrative application of the law to specific cases oftentimes extends beyond original timelines It may take several years for EPA to determine, based on observational data, that an air district is not in attainment with standards. For example, in the Bay Area, ozone concentration violations observed in 1995 eventually led to a new round of ozone attainment planning that produced a preliminary plan in 1999 and a more definitive plan in 2001, which was seven years after violations were detected. I echo Demerjian et al. (1995) who recommend that plan updates occur at shorter intervals (e.g., one, two or three years). These suggested assessments and, if necessary, adjustments should be based on a comparison of projected trends with air quality observations. When the two are found to be deviating, plan revisions are needed as soon as possible.

\section{Evidence of Change}

Criticism of the air quality planning process is in fact leading to changes at EPA and CARB. Respondents interviewed in the Central California case agreed that existing guidance for model performance evaluation is inadequate, and that new information is welcomed. CARB is updating its modeling guidance.

At EPA, two recent reports provide guidance for conducting air quality standard attainment modeling, and for science-based stakeholder processes. EPA has written guidance for using PAQSM to demonstrate attainment of the new 8-hour ozone standard 
(EPA 1999b). This is not to say that EPA has experienced a methodological and epistemological epiphany. The thoughtful suggestions of Demerjian et al. (1995) were received by EPA, but no action was taken. The 8-hour modeling guidance has languished in unquotable draft form for several years (Baldridge 2002).

Though ambitious, EPA's new modeling guidance falls short of the mark. It will not change the process substantively, though it incorporates methods to address several uncertainties. As shown in Figure IX-2, EPA proposes calculating a Relative Reduction Factor to manage the concern that modeled values might not be peak values of interest. It also allows for the incorporation of air quality model uncertainty analyses, but does not describe specifically how uncertainty information is to be used. As discussed in Chapter III - PAQSM and Their Uncertainties, such description remains an ongoing challenge and avenue of research.

As part of a weight-of-evidence demonstration, uncertainty is evaluated using corroborative modeling and data trends analyses determined on a case-by-case basis. On the one hand, this discussion of uncertainty is refreshing. Unfortunately, experiences from Central California do not bode well for the new approach. Weight-of-evidence has been similarly used in the past to obfuscate uncertainties rather than to deal with them explicitly. In this regard, there is no legal recourse, since courts assign to agencies the ultimate authority to rule on the weight-of-evidence. EPA's new guidance may only provided more layers of evidence that, though presumed informative, can create further barriers to legitimate understanding and explicit risk management. 
The EPA’s Science Advisory Board accepted the challenge of identifying strategies that "might allow [scientific and technical] knowledge to be better developed and used in [group stakeholder] decision processes" (Glaze and Morgan 2001). The objective of the report concurs with the recommendations of this research: using stakeholder process to make regulatory decisions, not just to provide input for regulators' decisions. The report begins with an optimistic, yet cautionary note, observing that the adequate treatment of science in stakeholder process is possible, but requires "substantial financial resources, adequate time, and high-quality staff" (SAB 2001). The report finds that there must be a shared commitment to explore the implications of all relevant science and to revisit problems as new information leads in unanticipated directions. It also notes that the setting must have a relatively small number of identifiable stakeholders, including those affected directly and indirectly by the problem, and that the legally responsible entity (e.g., EPA or CARB) must retain the right to review, modify or reject decisions. These findings support the recommendations of this chapter. The question remaining is how they might be incorporated into air quality planning.

The two EPA reports for science-based stakeholder processes and 8-hour ozone modeling are, together, promising steps in the right direction. EPA's SAB and OAQPS should be commended for tackling very difficult issues. Alas, one final and essential step still needs to be taken. Findings from this research highlight the need for the two EPA reports need to be integrated. This last step it is not apparently forthcoming. More needs to be done; hence the recommendations presented here. 
Figure IX-2: EPA's Draft 8-Hour Ozone

Attainment Demonstration Modeling Process (EPA 1999b)

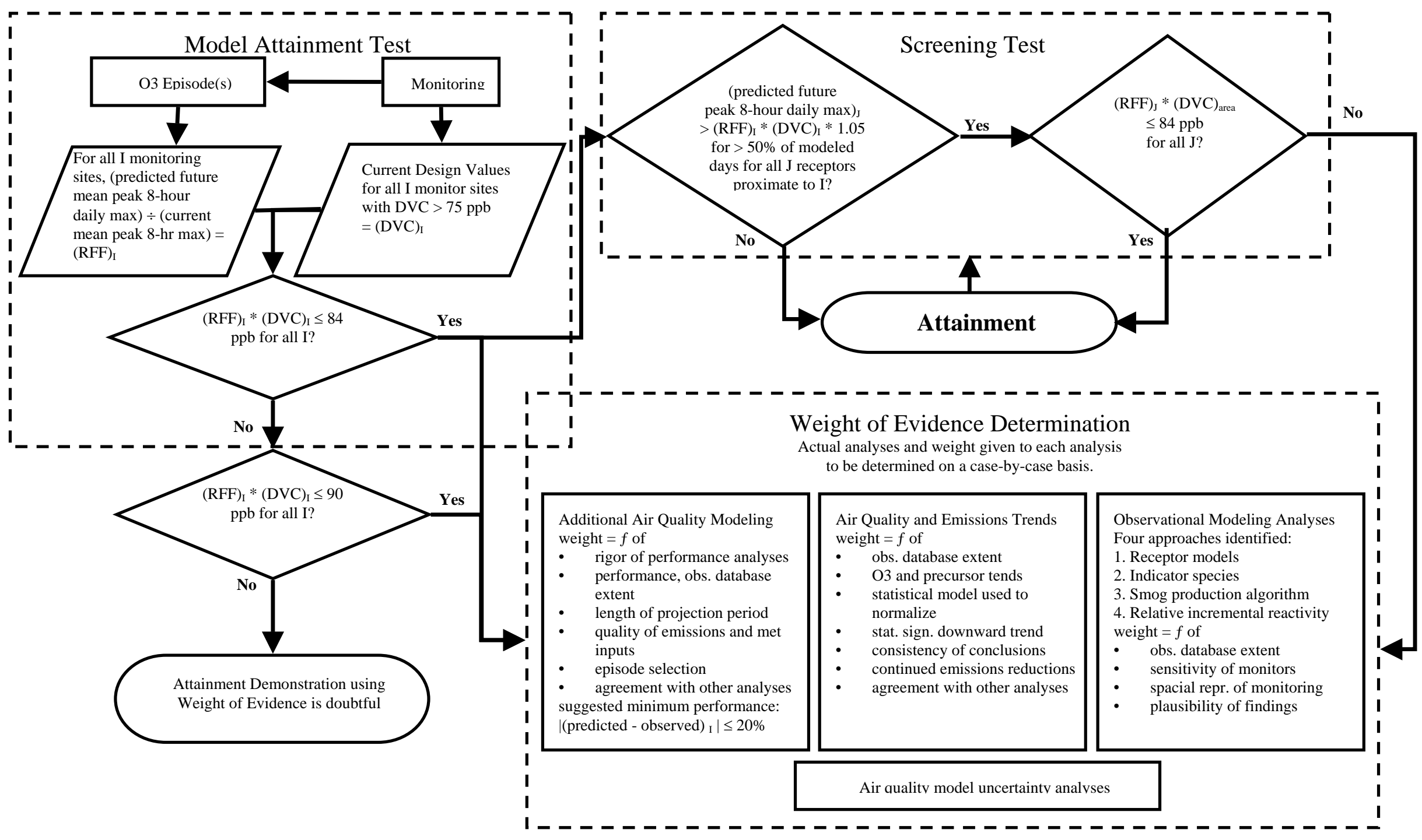




\section{BIBLIOGRAPHY}

(1955). Air Pollution Control Act.

(1963). Clean Air Act.

(1970). Federal Clean Air Act.

(1980). Comprehensive Environmental Response, Compensation and Liability Act.

(1990). Federal Clean Air Act.

(1998). WSPA Settlement Proposal - Revision Following April 10 Discussions, WSPA.

Webster's (1994). Webster's II New Riverside Dictionary, Houghton Mifflin Company.

ABAG (1978). San Francisco Bay Region - Environmental Management Plan. Oakland, Association of Bay Area Governments.

Abbaspour, K., R. Schulin, et al. (1999). “A Bayesian Approach for Incorporating Uncertainty and Data Worth in Environmental Projects.” Environ. Modeling and Assessment 1: 151-158.

Abstract, C. S. (2001). Average Maximum, Minimum and Annual Temperatures. Table A-5; Land and Water Areas of California, Table A-1, Voter Registration by County, California, October 5, 1998. Table O-3, California Department of Finance.

Anderson, B. (2001). 100 Attend Environmental Meeting. The Fresno Bee. Fresno.

Angel, B. (2001). Interview.

API (1989). Detailed Analysis of Ozone State Implementation Plans in Seven Areas Selected for Retrospective Evaluation of Reasons for State Implementation Plan Failure. Washington, D.C., American Petroleum Institute, Health and Environmental Sciences Department.

API (2002). San Joaquin Valley Sue California. Los Angeles Times. Los Angeles.

Arnstein, S. (1969). “A Ladder of Citizen Participation.” Jrnl. American Inst. of Planners 35(N4 July). 
Aronson, N. (1984). Science as a Claims-making Activity: Implications for Social Problems Research. Studies in the Sociology of Social Problems. J. Schneider and J. Kitsuse. Norwood, New Jersey, Ablex Publishing Corporation.

Ascher, W. (1976). Forecasting: An Appraisal for Policy-makers and Planners. Baltimore, Johns Hopkins University.

BAAQMD (1993). Local Air Quality Monitoring Plan. San Francisco, Bay Area Air Quality Management District.

BAAQMD (1997). 1997 Clean Air Plan, Volume I. San Francisco, Bay Area Air Quality Management District.

BAAQMD (2001a). San Francisco Bay Area Ozone Attainment Plan for the 1-hour National Ozone Standard. San Francisco, Bay Area Air Quality Management District in cooperation with Association of Bay Area Governments and Metropolitan Transportation Commission.

BAAQMD (2001b). Summer 2001 is Second Cleanest on Record. Air Currents, Bay Area Air Quality Management District. Fall.

Baldassare, M. (2001). PPIC Statewide Survey: Special Survey of the Central Valley. Sacramento, Great Valley Center and Public Policy Institute of California: 33.

Baldridge, E. (2002). Personal Communication., Environmental Protection Agency, Office of Air Quality Planning and Standards.

Bannock, G., R. Baxter, et al. (1998). The Penguin Dictionary of Economics. New York, John Wiley and Sons.

BEQ (2002). 2000-01 Annual Report, Appendix Table 14. Sacramento, California Board of Equalization.

Bergin, M., G. Noblet, et al. (1999). "Formal Uncertainty Analysis of a Lagrangian Photochemical Air Pollution Model.” Environ. Sci. \& Techn. 33(7): 1116-11126. 
Bergin, M. S., A. G. Russell, et al. (1995). "Quantification of individual VOC reactivity using a chemically detailed, three-dimensional photochemical model.” Environ. Sci. \& Technol.: 3029-3037.

Bergin, M. S., A. G. Russell, et al. (1998). "Effects of chemical mechanism uncertainties on the reactivity quantification of volatile organic compounds using a threedimensional air quality model.” Environ. Sci. \& Technol.: 694-703.

Bernard, H. (1995). Research Methods in Anthropology: Qualitative and Quantitative Approaches. Walnut Creek, Altamira Press, SAGE Publications.

Blanchard, C. (2000). “Ozone Process Insights From Field Experiments - Part III: Extent Of Reaction And Ozone Formation.” Atmos. Environ. 34(12-14): 2035-2043.

Blanchard, C. (2001). CCOS Data Analysis Observation-Based Assessment of VOC and NOx Limitation of Ozone Formation, Historical Analysis: 1990-2000.

Blanchard, C. and D. Fairley (2001). "Spatial Mapping of VOC and NOx-limitation of Ozone Formation in Central California.” Atmos. Environ. 35(22): 3861-3873.

Blanchard, C., F. Lurmann, et al. (1999). "The Use Of Ambient Data To Corroborate Analyses Of Ozone Control Strategies.” Atmos. Environ. 33(3): 369-381.

Blanchard, C., P. Roth, et al. (2000). “The Use Of Ambient Measurements To Identify Which Precursor Species Limit Aerosol Nitrate Formation.” Jrnl. Air \& Waste Mgmt. Assoc. 50(12): 2073-2084.

Blumenthal, D. L., T. B. Smith, et al. (1985). South San Joaquin Valley Ozone Study: Draft Final Report. Los Angeles, Western Oil \& Gas Association (WOGA).

Boyd, J. (1994). Preliminary Assessment of Transport on SJV Ozone, Air Resources Board.

Brewer, G. (1973a). Chapter 12: Dealing with Complex Social Problems: The Potential of the "Decision Seminar". Political Development and Change: A Policy Approach. G. Brewer and R.Brunner, Eds. New York, Collier Macmillan. 
Brewer, G. (1973b). Politicians, Bureaucrats, and Consultants: A Critique of Urban Problem Solving. New York, Basic Books.

Brown, T. C., G. L. Peterson, et al. (1995). “The Values Jury to Air Natural Resource Decisions.” Land Economics 71(2): 250-260.

Bryner, G. (1993). Blue Skies, Green Politics: The Clean Air Act of 1990. Washington, D.C., CQ Press.

Byun, D., C. Coats, et al. (1995). Prototyping and Implementation of Multiscale Air Quality Models for High Performance Computing. SCS 1995 Simulation MultiConference, Phoenix, AZ.

CAAA (1990). Federal Clean Air Act. Public Law 91-604, 42 U.S.C. 7513b.

Calvert, J., J. Heywood, et al. (1993). “Achieving Acceptable Air Quality: Some Reflections on Control Vehicle Emissions.” Science 261(517 (July 2)): 37-45.

CARB (1990). Assessment and Mitigation of the Impacts of Transported Pollutants on Ozone Concentrations Within California. Sacramento, Technical Support Division and Office of Air Quality Planning and Liaison, California Air Resources Board.

CARB (1992). Technical Guidance Document: Photochemical Modeling, California Environmental Protection Agency and California Air Resources Board.

CARB (1993). Guidance for Annual and Triennial Progress Reports Under the California Clean Air Act. Sacramento, California Air Resources Board.

CARB (1994). California State Implementation Plan. Volume I. Sacramento, Office of Air Quality \& Transportation Planning. California Air Resources Board.

CARB (1996a). Final Statement of Reasons for Rulemaking Including Summary of Comments and Agency Response for Triennial Review of the Assessment and Mitigation of the Impacts of Transported Pollutants on Ozone Concentrations Within California. Sacramento, Technical Support Division, California Air Resources Board. CARB (1996b). Performance Evaluation of SAQM in Central California and Attainment Demonstration for the August 3-6, 1990 Ozone Episode. Sacramento, Modeling 
Support Section, Modeling and Meteorology Branch, Technical Support Division, California Air Resources Board.

CARB (1997). . Sacramento, California Air Resources Board, Technical Support Division.

CARB (2001). 94 SIP vs Bump-UP SIP SJV Emissions. Sacramento, California Air Resources Board.

CARB (2001). Indoor Air Quality: Residential Cooking Exposures. Final Report. Sacramento, California Air Resources Board, Research Division.

CARB (2001). Ozone Transport: 2001 Review. Sacramento, California Air Resources Board: 57.

CARB (2001). Study Overview of the California Regional Particulates Air Quality Study. Sacramento, California Air Resources Board.

CARB (2002). 2002 Almanac Data: Oxides of Nitrogen Projected Emission Inventory: San Joaquin Valley Unified APCD, http://www.arb.ca.gov/emisinv/emsmain/emsmain.htm. 2003.

CARB/CalEPA (2002). Proposed Clean Air Plan: Strategies for a Health Future: Executive Summary. Sacramento, California Environmental Protection Agency, California Air Resources Board.

Casman, E., G. Morgan, et al. (1999). “Mixed Levels of Uncertainty in Complex Policy Models.” Risk Analysis 19(1): 33-42.

CCAA (2001). California Health And Safety Code. Division 26. Air Resource. Part 3. Chapter 10. Sacramento, California Air Resources Board.

CCOS (1999). Draft Central California Ozone Study Scope of Work. Sacramento, California Air Resources Board.

Census (2000). 2000 Summary File 1; Population of Counties by Decennial Census. Washington, D.C., U.S. Bureau of the Census, Population Division, Richard Forstall. 
Cleary, R. (1976). Mathematical Models. Boundaries of Analysis. Cambridge, Ballinger Publishing Company.

Coe, D. (1998). Technical Support Study 15: Evaluation and Improvement of Methods For Determining Ammonia Emissions in the San Joaquin Valley. Final Report. Sacramento, California Air Resources Board.

Condit, G. (1996). Letter Responding to Marcus letter of Aug 29, 1996, Congress of U.S., House of Representatives.

Covell, N. (1998). Request for Support by the WSPA for Proposed Alternative to Legally Required Transport Mitigation, Sacramento Metropolitan Air Quality Management District.

Cowling, E. (1992). “The Performance and Legacy of NAPAP.” Ecological Applications 2(2): 111-116.

Crow, D. (1997). Questions related to modeling, Questions related to the Proposed Alternative, and Other Related Questions, SJVUAPCD.

Crow, D. (1997). SJVUAPCD Concerns about alternatives to BARCT controls on Bay Area refineries discussed at July 15, 1997 meeting, SJVUAPCD.

CRPAQS unpublished data, California Regional Particulate Air Quality Study.

Dabdub, D., L. DeHaan, et al. (1999). "Analysis of Ozone in the San Joaquin Valley of California.” Atmos. Environ. 33: 2501-2514.

Dabdub, D. and J. H. Seinfeld (1995). "Extrapolation techniques used in the solution of stiff ODEs associated with chemical kinetics of air quality models.” Atmos. Environ. 29: 403-410.

Dalkey, N. (1968). The Delphi Method: An Experimental Study of Group Opinion. Santa Monica, The Rand Corporation.

Davis, G. (1999). Governor's Address at Central Valley Economic Summit, State of California. 
Demerjian, K., P. Roth, et al. (1995). A New Approach for Demonstrating Attainment of the Ambient Ozone Standard. Research Triangle Park, NC, Office of Research and Development. U.S. Environmental Protection Agency.

Dennis, R., D. Byun, et al. (1996). “The Next Generation of Integrated Air Quality Modeling: EPA's Models-3.” Atmos. Environ. 30(12): 1925-1938.

Doris, L. and F. Wicher (1998). Technical Support Document for U.S. EPA's Proposed Federal Implementation Plan for the Phoenix Nonattainment Area - RACM/RACT Analysis. San Francisco, U.S. Environmental Protection Agency, Region IX, Air Division.

DOT (1999). California Motor Vehicle Stock, Travel and Fuel Forecast. Sacramento, California Department of Transportation.

Dutton, W. and K. Kraemer (1985). Modeling as Negotiating: The Political Dynamics of Computer Models in the Policy Process. Norwood, NJ, Ablex Publishing Corp.

Dwyer, J. (1990). “The Pathology of Symbolic Legislation.” Ecology Law Quarterly 17(2): 233-316.

EPA (1987). Guideline on Air Quality Models (Revised) and Supplement A. Research Triangle Park, U.S. Environmental Protection Agency.

EPA (1991). Guideline for Regulatory Application of the Urban Airshed Model.

Research Triangle Park, U.S. Environmental Protection Agency.

EPA (1992a). Protocol for Determining the Best Performing Model. Research Triangle

Park, U.S. Environmental Protection Agency, Office of Air Quality Planning and Standards.

EPA (1992b). State Implementation Plans; Nitrogen Oxides Supplement to the General Preamble for the Implementation of Title I of the Clean Air Act Amendments of 1990, United States Environmental Protection Agency.

EPA (1993a). Guidance for Growth Factors, Projections and Control Strategies for the 15 Percent Rate-of-Progress Plans. Research Triangle Park, U.S. Environmental Protection Agency. 
EPA (1993b). User's Guide for the Urban Airshed Model, Volume IV. Research Triangle Park, U.S. Environmental Protection Agency, Office of Air Quality Planning and Standards.

EPA (1996a). Appendix W to Part 51 - Guideline on Air Quality Models. Research Triangle Park, Federal Register.

EPA (1996b). Guidance on the Use of Modeled Results to Demonstrate Attainment of the Ozone NAAQS. Research Triangle Park, U.S. Environmental Protection Agency, Office of Air Quality Planning and Standards.

EPA (1997a). Final Revisions to the Ozone and Particulate Air Quality Standards. Washington, D.C., U.S. Environmental Protection Agency, Office of Air And Radiation.

EPA (1997b). Guidance for Emissions Inventory Development. Research Triangle Park, U.S. Environmental Protection Agency.

EPA (1997c). Proposed Regional Haze Regulations. Washington, D.C., U.S. Environmental Protection Agency, Office of Air And Radiation.

EPA (1999a). "Classification of the San Francisco Bay Area Nonattainment Area for the Congestion Mitigation and Air Quality (CMAQ) Purposes.” Federal Register 64(52): 13383-13384.

EPA (1999b). Draft Guidance on the Use of Models and Other Analyses in Attainment Demonstrations for the 8-Hour Ozone NAAQS. Research Triangle Park, U.S. Environmental Protection Agency, Office of Air Quality Planning and Standards.

EPA (1999c). Emissions Inventory Guidance for Implementation of Ozone and Particulate Matter National Ambient Air Quality Standards (NAAQS) and Regional Haze Regulations. Research Triangle Park, U.S. Environmental Protection Agency, Office of Air Quality Planning and Standards.

EPA (1999d). Final Regional Haze Regulations. Washington, D.C., U.S. Environmental Protection Agency, Office of Air And Radiation. 
EPA (2001). National Air Quality and Emissions Trends Report, 1999. Research Triangle Park, United States Environmental Protection Agency, Office of Air Quality Planning and Standards.

EPA (2002). National Air Quality and Emissions Trends Report, 2000, Appendix A, Tables A-15 and A-19. Research Triangle Park, U. S. Environmental Protection Agency, Office of Air Quality Planning and Standards.

EPA (2003). Regulatory Announcement: Agricultural Pump and Irrigation Rule Amending the Nonroad Engine Definition. Research Triangle Park, U. S. Environmental Protection Agency, Air and Radiation Division, Office of Transportation and Air Quality.

Epstein, S. (1996). Impure Science. Berkeley, University of California Press.

Fairley, D. (1996). Analysis of Population Exposure to Ozone under the 1991 CAP with and without Refinery NOx rule, BAAQMD.

Fine, J. and P. Roth (1996). Evaluation of the Feasibility of Identifying the Influence of Biogenic Emissions on Air Quality in the San Francisco Bay Area. Causes of Recent Exceedences of the Ozone Standard In The San Francisco Bay Area. Project 3: Biogenic Emissions. Task 1 Report. San Rafael, CA, Envair.

Fine, J., L. Vuilleumier, et al. (2003). "Evaluating Uncertainties in Regional Photochemical Air Quality Modeling.” Ann. Rvw. Energy and Resources 28(in review).

Finkel, A. (1990). Confronting Uncertainty in Risk Management: A Guide to DecisionMakers. Washington, D.C., Center for Risk Management, Resources For The Future.

Fischer, F. (1990). Technocracy and the Politics of Expertise. Newbury Park, SAGE Publications.

Fischer, F. and J. Forester, Eds. (1993). The Argumentative Turn in Policy Analysis and Planning., Duke University Press.

Federal Register (1993). Reclassification of Moderate PM-10 Nonattainment Areas to Serious Areas - Part II. Washington, D.C., Federal Register: 3334-. 
Federal Register (1999). Classification of the San Francisco Bay Area Ozone

Nonattainment Area for Congestion Mitigation and Air Quality (CMAQ)

Improvement Program Purposes. Washington, D.C., Federal Register: 39416-39418.

Federal Register (1999). Regional Haze Regulations, Final Rule. Washington, D.C.,

Federal Register: 40 CFR Part 51.

Federal Register (2001). Clean Air Act Reclassification, San Joaquin Valley

Nonattainment Area; Designation of East Kern County Nonattainment Area and

Extension of Attainment Date; California; Ozone. Washington, D.C., Federal Register: 56476-56484.

French, B. (1993). "More Effective Citizen Participation in Environmental DecisionMaking.” University of Toledo Law Review 24(Winter): 389-423.

Funtowicz, S. and J. Ravetz (1990). Uncertainty and Quality in Science for Public Policy, Dordrecht: Kluwer Academic Publishers.

Funtowicz, S. and J. Ravetz (1992). Three Types of Risk Assessment and the Emergence of Post-Normal Science. Social Theories of Risk. S. K. a. D. Golding., Westport, Praeger.

Geron, C., A. Guenther, et al. (1994). "An improved model for estimating emissions of volatile organic compounds from forests in the Eastern United States.” Jrnl. Geophy. Res. 99: 12773-91.

Gery, M. W., G. Z. Whitten, et al. (1989). "A photochemical kinetics mechanism for urban and regional scale computer modeling.” Jrnl. Geophy. Res. 94(D10): 12,92512,956 .

Glaze, W. and G. Morgan (2001). Improved Science-based Environmental Stakeholder Processes: An EPA Science Advisory Board Commentary, Environmental Protection Agency, Office of the Administrator, Science Advisory Board.

Greenberger, M., M. Crenson, et al. (1976). Models in the Policy Process: Public Decision Making in the Computer Era. New York, Russell Sage Foundation. 
Gunther, A. (1982). An Introduction to the SAI Airshed Grid Model and It's Application to California's South Coast Air Basin. Energy and Resources Group. Berkeley, University of California.

GVC (1999). The State of the Great Central Valley of California. Modesto, Great Valley Center.

Hajer, M. (1993). Discourse Coalitions and the Institutionalization of Practice: The Case of Acid Rain in Great Britain. The Argumentative Turn in Policy Analysis and Planning. F. a. J. F. Fischer. Durham, Duke University Press.

Hall, S., P. Matson, et al. (1996). "NOx Emissions for Soil: Implications for Air Quality Modeling in Agricultural Regions.” Ann. Rvw. Energy and the Environment 21.

Hanna, S. R., J. C. Chang, et al. (1998). “Monte Carlo estimates of uncertainties in predictions by a photochemical grid model (UAM-IV) due to uncertainties in input variables." Atmos. Environ. 32: 3619-3628.

Hanna, S. R., Z. Lu, et al. (2001). "Uncertainties in Predicted Ozone Concentrations Due to Input Uncertainties for the UAM-V Photochemical Grid Model Applied to the July 1995 OTAG Domain.” Atmos. Environ. 35(891-903).

Hansen, A. (2000). Uncertainties in Predicted Ozone Concentrations Due to Input Uncertainties for the UAM-V Photochemical Grid Model Applied to the July 1995 OTAG Domain. Palo Alto, California and St. Louis, Missouri, EPRI.

Harley, R., R. Sawyer, et al. (1997). “Updated photochemical modeling for California's South Coast Air Basin: Comparison of chemical mechanisms and motor vehicle emissions inventories.” Environ. Sci. \& Technol. 31: 135-154.

Heim, M. (1998). County Population Projections with Age, Sex and Race/Ethnic Detail. Sacramento, State of California, Department of Finance.

Helmer, O. (1963). The Systematic Use of Expert Judgment in Operations Research. Santa Monica, The Rand Corporation.

Holling, C., Ed. (1978). Adaptive Environmental Assessment and Management. New York, John Wiley Publishing. 
Howekamp, D., J. Boyd, et al. (1994). Letter of Intent Concerning the Dynamic and Evolving Content of the San Joaquin Valley Air Quality Attainment Plan.

Hyink, B., S. Brown, et al. (1959). Politics and Government in California. New York, Tomas Y. Crowell Company.

Innes, J. (1998). “Information in Communicative Planning.” American Planning Association Jrnl. Winter: 52-63.

Jager, J. (1998). “Current Thinking on Using Scientific Findings in Environmental Policy Making.” Environ. Modeling and Assessment 3: 143-153.

Jang, J.-C. C., H. E. Jeffries, et al. (1995). "Sensitivity of ozone to model grid resolution II. Detailed process analysis for ozone chemistry.” Atmos. Environ. 29: 3101-3114.

Jasanoff, S. (1990). The Fifth Branch. Cambridge, Harvard University Press.

Jasanoff, S. (1995). Skinning Scientific Cats. Green Planet Blues. K. Conca, M. Alberty and G. Dabelko. Boulder, CO, Westview Press.

Jasanoff, S. (1996a). "Beyond Epistemology: Relativism and Engagement in the Politics of Science.” Social Studies Sciences 26.

Jasanoff, S. (1996b). Science at the Bar: Law, Science and Technology in America. Cambridge, Harvard University Press.

Jeffries, H. E. and S. Tonnesen (1994). "A comparison of two photochemical reaction mechanisms using a mass balance and process analysis.” Atmos. Environ. 28: 29913003.

Jenkins, P., T. Phillips, et al. (1992). “Activity Patterns of Californians: Use of and Proximity to Indoor Pollutant Sources.” Atmos. Environ. 26A(12): 2141-2148.

Jones, E. (1997). "Risky Assessments: Uncertainties In Science And The Human Dimensions Of Environmental Decision-making.” William and Mary Environmental Law and Policy Review(Fall).

Jordan, D. (1998). Regarding NOx Rules Exemptions for Facilities West of I-5, U.S. Environmental Protection Agency, Region IX, Air Division. 
Kaduwela, A. (1996). O3 Isopleths for Selected Monitoring Stations: 1990 Base Case (Draft), California Air Resources Board.

Kagan, R. (1991). “Adversarial Legalism and American Government.” Jrnl. of Policy Analysis and Mgmt. 10(3): 369-406.

Kenny, M. (1998). Response to Request by SJVUAPCD NOx Subcommittee for CARB's Input, California Air Resources Board.

Korfmacher, K. (1998). “Water Quality Modeling for Environmental Management: Lessons from the Policy Sciences.” Policy Sciences 31: 35-54.

Kuhn, T. (1962). The Structure of Scientific Revolutions, University of Chicago Press.

Kuminoff, N., D. Sumner, et al. (2000). The Measure of California Agriculture, 2000. Davis, University of California Agricultural Issues Center.

Lamanna, M. S. and A. H. Goldstein (1999). “In situ measurements of C2-C10 volatile organic compounds above a Sierra Nevada ponderosa pine plantation.” Jrnl. Geophy. Res. 104: 21,247-21,262.

Landy, M., M. Roberts, et al. (1990). The Environmental Protection Agency: Asking the Wrong Questions. New York, Oxford University Press.

Lee, K. (1993). Compass and Gyroscope: Integrating Science and Politics for the Environment. Washington, D.C., Island Press.

Lehrman, D., T. Smith, et al. (1998). Meteorological Representativeness and Fog and Low Clouds Characteristics. California Regional Particulate Air Quality Study. 1995 Integrated Monitoring Study Data Analysis. Final Report. Santa Rosa, Technical Support Division, California Air Resources Board.

Lipsky, M. (1980). Street-Level Bureaucracy. New York, Russel Sage Foundation. Lunden, M., T. Thatcher, et al. (2003). “The Use of Time and Chemically-Resolved Particle Data to Characterize the Infiltration of Outdoor PM2.5 into a Residence in the San Joaquin Valley.” Environ. Sci. \& Technol. (in review). 
Lurmann, F., W. Carter, et al. (1987). A surrogate species chemical reaction mechanism for urban-scale air quality simulation models, vols. I and II. Research Triangle Park, N.C., U.S. Environmental Protection Agency.

Magliano, K. (1998). Chemical Mass Balance Modeling of Data From the 1995 Integrated Monitoring Study. Final Report. Sacramento, California Regional PM10/PM2.5 Air Quality Study and California Air Resources Board.

Mahlman, J. (1998). “Science and Nonscience Concerning Human-Caused Climate Warming.” Ann. Rvw. Energy \& Environ. 23: 83-106.

Majone, G. (1989). Evidence, Argument and Persuasion in the Policy Process. New Haven, Yale University Press.

Manning, P. and B. Cullum-Swan (1998). Content and Narrative Analysis. Collecting and Interpreting Qualitative Materials. N. a. Y. L. Densin. Sage Publications, Beverly Hills.

Marr, L., D. Black, et al. (2002a). "Formation of photochemical air pollution in Central California: 1. Development of a revised motor vehicle emission inventory.” Jrnl. Geophy. Res. 107(6).

Marr, L. C. (2002b). Changes in Ozone Sensitivity to Precursor Emissions on Diurnal, Weekly, and Decadal Time Scales. Civil and Environmental Engineering. Berkeley, University of California at Berkeley.

Martien, P. and T. Umeda (1993). Technical Memorandum: Photochemical Model Sensitivity Tests of the Effects of Utility Boiler NOx Controls on Ambient Ozone Concentrations. San Francisco, Bay Area Air Quality Management District, Planning and Research Divisions.

Martien, P., T. Umeda, et al. (1992). Technical Memorandum: Photochemical Model Sensitivity Tests of the Effects of NOx Controls for Non-Utility Boilers on Ambient Ozone Concentrations. San Francisco, Bay Area Air Quality Management District, Planning and Research Divisions. 
Matson, P. (1997). "NOx emission from soils and its consequences for the atmosphere and biosphere - critical gaps and research directions for the future.” Nutrient Cycling in Agroecosystems 48(1-2): 1-6.

Mearer, T. (1994). Bay Area Smog Drifts into Kern. Bakersfield Californian. Bakersfield: 2.

Menon, S., J. Hansen, et al. (2002). "Climate Effects of Black Carbon Aerosols in China and India.” Science 297(September 27): 2259-2253.

Merchen, J. (2002). Mobile Source Emissions Inventory Update Using EMFAC 2001 v2.08. Fresno, San Joaquin Valley Unified Air Pollution Control District.

Miles, M. and A. M. Huberman (1994). Qualitative Data Analysis: A Sourcebook of New Methods. Beverly Hills, Sage Publications.

Milford, J. B., D. Gao, et al. (1994). "Total reactive nitrogen ( $\mathrm{NO}_{\mathrm{y}}$ ) as an indicator of the sensitivity of ozone to reductions in hydrocarbon and $\mathrm{NO}_{\mathrm{x}}$ emissions.” Jrnl. Geophys. Res. 99: 3533-3542.

Milford, J. B., A. G. Russell, et al. (1989). “A new approach to photochemical pollution control: implications of spatial patterns in pollutant responses to reductions in nitrogen oxides and reactive organic gas emissions.” Environ. Sci. \& Technol. 23: 1290-1301.

Morgan, M. G. (2001). “The Neglected Art of Bounding Analysis.” Environ. Sci. \& Techn. 35: 162A-164A.

Morgan, M. G. and M. Henrion (1990). Uncertainty: A Guide to Dealing with Uncertainty in Quantitative Risk and Policy Analysis. Cambridge, Cambridge University Press.

Morris, R., T. Myer, et al. (1991). Development of a nested-grid urban airshed model and application to Southern California. Air and Waste Management Association meeting, Vancouver, B.C., Canada. 
Munroe, T., J. Anguiano, et al. (2001). Economic Forecast for California's Central Valley. Modesto, Munroe Consulting Inc. and New Economic Forecasting for Great Valley Center: 67.

NARSTO (2000a). An Assessment of Tropospheric Ozone Pollution: A North American Perspective., North American Research Strategy for Tropospheric Ozone (NARSTO).

NARSTO (2000b). Chapter 4: The Air-Quality Modeling System. An Assessment of Tropospheric Ozone Pollution: A North American Perspective., North American Research Strategy for Tropospheric Ozone (NARSTO).

NARSTO (2003). Particulate Matter Science for Policy Makers: A NARSTO Assessment, Part 2, NARSTO.

Naylor, T.; and J. Finger (1967). "Verification of Computer Simulation Models.” Mgmt. Sci. 14(2: October): B-92.

NRC (1991). Rethinking the Ozone Problem in Urban and Regional Air Pollution. Washington, D.C., National Research Council, National Academy Press.

Odman, M. and A. Russell (1991). "Multiscale modeling of pollutant transport with chemistry.” Jrnl. Geophys. Rsrch. 96: 7363-7370.

Oreskes, N., K. Shrader-Frechette, et al. (1994). "Verification, Validation, and Confirmation of Numerical Models in the Earth Sciences.” Science 263(Feb. 4): 641646.

OTA (1989). Catching Our Breadth: Next Steps for Reducing Urban Ozone. Washington, D.C., Office of Technology Assessment.

OTAG (1997). Final Report, Vol. I: Executive Summary, Ozone Transport Assessment Group Air Quality Analysis Workgroup, Dave Guinnup and Bob Collom, Co-chair.

Ozawa, C. (1991). Recasting Science: Consensual Procedures in Public Policy Making. Boulder, Westview Press.

Ozawa, C. (1996). "Science in Environmental Conflicts.” Sociological Perspectives 39(2): 219-230. 
Parsons, J. (1986). A Geographer Looks at the San Joaquin Valley, Carl O. Sauer

Memorial Lecture, Alumni House, University of California.

Pattison, K. (1996). California Smog Plan Drives Motorists to Protest. The Christian Sci. Monitor.

Penbera, J. (1996). Economic Map: A Statistical Abstract of the Central Valley. Fresno, Central California Futures Institute.

Pierson, W., A. Gertler, et al. (1990). "Comparison of the SCAQS tunnel study with other on-road vehicle emissions data.” Jrnl. Air \& Waste Mgmt.. Assoc. 40: 1495-1504.

Polakovic, G. (2001). California's Smog Story is Tale of 2 States. Los Angeles Times. Los Angeles.

Pool, R. (1997). Beyond Engineering: How Society Shapes Technology. Oxford, Oxford University Press.

Popovic, N. (1993). “The Right To Participate in Decisions That Affect the Environment.” Pace Environmental Law Review 10(2, Spring): 683-709.

Pun, B. and C. Seigneur (1998). Conceptual Model of Particulate Matter Pollution in the California San Joaquin Valley. San Ramon, California, Atmospheric and Environmental Research, Inc.

Quinn, T. A. (2001). The San Francisco Bay Area Petroleum Industry: Economic Impact, Community Value. Glendale, Western States Petroleum Association.

Ramo, A. (2001). Interview.

Ranzieri, A. (1996). Bay-Area Boiler/Heater NOx Reduction Simulations: Update for the Meeting on Sept 9, 1996, California Air Resources Board.

Ravetz, J. (1999). "Developing Principles of "Good Practice" in Integrated Environmental Assessment.” International Jrnl. of Environ. \& Pollution 11(3).

Reheis, C. (1998). WSPA Settlement Proposal, Western States Petroleum Association.

Reheis-Boyd, C. H. (2002). Letter to Sam Armentrout, Mayor of City of Madera and Chairman of the SJVUAPCD, San Joaquin Valley Air Quality Coalition. 
Reynolds, S., C. Blanchard, et al. (2002). "Understanding the Effectiveness of Precursor Reductions in Lowering 8-Hr Ozone Concentrations.” Jrnl. Air \& Waste Mgmt. Assoc. 53(February): 195-205.

Rittel, H. and M. Webber (1973). "Dilemmas in a General Theory of Planning.” Policy Sciences 4 .

Roe, E. (1994). Narrative Policy Analysis: Theory and Practice. Durham, Duke University Press.

Rosaldo, R. (1992). Culture and Truth: The Remaking of Social Analysis. Boston, Beacon Press.

Roth, P. (1999). “A Qualitative Approach to Evaluating the Anticipated Reliability of a Photochemical Air Quality Simulation Model for a Selected Application.” Jrnl. Air \& Waste Mgmt. Assoc. 49: 1050-1059.

Roth, P., C. Blanchard, et al. (1989). The Role of Grid-Based, Reactive Air Quality Modeling in Policy Analysis: Perspectives and Implications, As Drawn From a Case Study, Atmospheric Research and Exposure Assessment Laboratory, Office of Research and Development, U.S. Environmental Protection Agency.

Roth, P., T. Tesche, et al. (1997). A Critical Review of Regulatory Air Quality Modeling for Tropospheric Ozone. Washington, D.C., American Petroleum Institute for the North American Research Strategy for Tropospheric Ozone (NARSTO).

Roth, P., S. Ziman, et al. (1993). Tropospheric Ozone. Keeping Pace With Science and Engineering: Case Studies in Environmental Regulation. Washington, D.C., National Academy of Engineering.

Roth, P. M., S. D. Reynolds, et al. (1998). Protocol for evaluating the performance of the SARMAP model. San Anselmo, Envair.

Russell, A. (1997). “Regional Photochemical Air Quality Modeling: Model Formulations, History, and State of the Science.” Ann. Rvw. Energy \& Environ. 22: 537-588. 
Russell, A. and R. Dennis (2000). "NARSTO critical review of photochemical models and modeling.” Atmos. Environ. 34: 2283-2324.

SAB (2001). Improved Science-Based Environmental Stakeholder Processes. Washington DC, Environmental Protection Agency Science Advisory Board.

Sarewitz, D., R. A. J. Pielke, et al., Eds. (2000). Prediction: Science, Decision Making, and the Future of Nature. Washington, D.C., Island Press.

Sawyer, R., R. Harley, et al. (2000). "Mobile sources critical review: 1998 NARSTO Assessment.” Atmos. Environ. 34: 2161-2181.

SBCAPCD (1999). California's Air Pollution Future: ARB's New Chair Talks about the Tough Problems and the Promising Technology Solutions Ahead. On the Air. Santa Barbara.

Schade, G., A. Goldstein, et al. (1999). “Are monoterpene emissions influenced by humidity?” Geophys. Res. Ltrs. 26(14): 2187-2190.

Schumacher, E. F. (1973). Small Is Beautiful: Economics as if People Mattered. London, Blond and Briggs, Ltd.

Seaman, N. L. (2000). "Meteorological modeling for air-quality assessments." Atmos. Environ. 34: 2231-2259.

Seinfeld, J. and S. Pandis (1998). Atmospheric Chemistry and Physics. New York, John Wiley and Sons, Inc.

Shackley, S. and B. Wynne (1996). " Representing Uncertainty in Global Climate Change Science and Policy: Boundary-Ordering Devices and Authority.” Sci., Technology and Human Values 21(3): 275-302.

Sher, B. (1998). WSPA's Alternative Compliance Proposal for BAAQMD Reg IX, Rule 10, California State Senate.

Sherman, J. et al. (1997). Finding Invisible Farm Workers: The Parliear Survey, California Institute of Rural Studies. 
Simpson, D., A. Guenther, et al. (1995). "Biogenic emissions in Europe. 1. estimates and uncertainties.” J. Geophys. Res. 100: 22875-90.

Singer, B. and R. Harley (1996). “A Fuel-Based Motor Vehicle Emissions Inventory.” Jrnl. Air \& Waste Mgmt. Assoc. 46: 581-593.

Singer, B. and R. Harley (2000). “A Fuel-Based Inventory of Motor Vehicle Exhaust Emissions in the Los Angeles area during Summer 1997.” Atmos. Environ. 34: 17831795.

Sistla, G., N. Zhou, et al. (1996). “Effects of Uncertainties in Meteorological Inputs on Urban Airshed Model Predictions and Ozone Control Strategies.” Atmos. Environ. 30(12): 2011-2025.

SJVAQS (1996). San Joaquin Valley Air Quality Study Policy-Relevant Findings. Fresno, Policy Committee, San Joaquin Valley Air Quality Study, California Air Resources Board and San Joaquin Valley Unified Air Pollution Control District.

SJVUAPCD Valley Air District, San Joaquin Valley Unified Air Pollution Control District.

SJVUAPCD (1994). The Ozone Attainment Demonstration Plan. Fresno, San Joaquin Valley Unified Air Pollution Control District.

SJVUAPCD (1997). PM-10 Attainment Demonstration Plan. Fresno, San Joaquin Valley Unified Air Pollution Control District.

SJVUAPCD (2001a). California Clean Air Act Triennial Progress Report and Plan Revision 1997-1999. Fresno, San Joaquin Valley Unified Air Pollution Control District.

SJVUAPCD (2001b). Draft 2002 Amendment to the San Joaquin Valley Ozone Plan. Fresno, San Joaquin Valley Unified Air Pollution Control District. SJVUAPCD (2001c). Ramifications of Pursuing Severe or Extreme Attainment Demonstration Tract. Fresno, San Joaquin Valley Unified Air Pollution Control District. 
SJVUAPCD (2002). Media Release: Valley Air district To Sue State Over Smog Drift from Bay Area, San Joaquin Valley Unified Air Pollution Control District.

SJVUAPCD (2003). News Release: Decades of negotiations end in EPA support of local air quality rule, State put on notice that agricultural exemption must be axed., San Joaquin Valley Unified Air Pollution Control District.

SMAQMD (2002). SMAQMD Sues EPA Over Bay Area Air Pollution Impact on our Region, Sacramento Metropolitan Air Quality Management District.

Stake, R. E. (2000). Case Studies. Handbook of Qualitative Research, Second Edition. N. K. D. a. Y. S. Lincoln. Thousand Oaks, Sage Publications.

Stauffer, D. and N. Seaman (1990). "Use of four dimensional data assimilation in a limited area mesoscale, part I: experiments with synoptic data.” Mon. Weather Rev. 118: $1250-77$.

Sterner, R. The Great Valley. Baltimore, Johns Hopkins Applied Physics Lab.

Stokey, E. and R. Zeckhauser (1978). A Primer for Policy Analysis. New York, Norton and Company.

TAC (2001). Vision: Develop and Implement an Aerometric Monitoring Network for Central California to Enhance Effective Air Quality Management for Criteria Air Pollutants and Air Toxics and Track Progress Towards Meeting Regulatory Requirements, Technical Advisory Committee, Central California Ozone Study.

Tanner, R. (1992). Emission Data Collection and Inventory Development, Work Element 2: Development of a Natural Source Emissions Inventory. Reno, Desert Research Institute. Energy and Environmental Engineering Center.

Tesche, T. (1988). “Accuracy of Ozone Air Quality Models.” Jrnl. of Environ. Engineering 114.

Tuinstra, W., L. Hordjik, et al. (1999). "Using Computer Models in International Negotiations.” Environment 41(9): 33-42. 
Umbach, K. (1997). A Statistical Tour of California's Great Central Valley. Sacramento, California Research Bureau: 47.

Unknown (1998). Review of Air Quality in the San Francisco Bay Area for Selected Stations for 1995-1997, WSPA or Chevron or?

USDOA (1997). Census of Agriculture, Geographic Area Series, Table 1. County Summary Highlights: 1997, United States Department of Agriculture, California Agricultural Statistics Service.

Van der Sluijs, J. NUSAP.net, Copernicus Institute for Sustainable Development and Innovation,. 2002a.

Van der Sluijs, J., J. Risbey, et al. (2002b). Uncertainty Assessment of VOC Emissions from Paint in the Netherlands, Copernicus Institute for Sustainable Development and Innovation, Department of Science, Technology and Society, Utrecht University.

VanZam, P. (1998). WSPA's Proposed Relaxation of BARCT for Refinery Boilers, Northern Sierra AQMD.

Vuilleumier, L., N. J. Brown, et al. (2000). Review and Improvement of Methods for Estimating Rates of Photolysis in Photochemical Models. Volume II: User's Guide for TUVAQM Radiative Transfer and Photolysis Module. Berkeley, Lawrence Berkeley National Laboratory.

Vuilleumier, L., R. A. Harley, et al. (2001). "Variability in ultraviolet total optical depth during the Southern California Ozone Study (SCOS97).” Atmos. Environ. 35: 11111122.

Walters, C. and C. Holling (1990). "Large-scale Management Experiments and Learning by Doing." Ecology 71(6 (December)).

Wang, M. (1993). Comments on ARB Workshop on Transport Couples, Western States Petroleum Association.

Weinberg, A. (1972). “Science and Trans-Science.” Minerva 10(April): 209-222. 
Wesely, M. L. and B. B. Hicks (2000). "A review of the current status of knowledge on dry deposition.” Atmos. Environ. 34: 2261-2282.

Whitten, G. Z., T. C. Meyers, et al. (1985). Application of the Urban Airshed Model to Kern County. Final Report. San Rafael, Systems Applications, Inc., Prepared for Western Oil \& Gas Association, Los Angeles.

WHO (2000). Air Pollution, World Health Organization: http://www.who.int/inffs/en/fact187.html, last visited April 26, 2003.

Wilson, E., M. Oldfield, et al. (2003). 2001 California Oil and Gas Production Report. Sacramento, California Department of Conservation: January 28.

Winer, A. (1996). Critical Evaluation of a Biogenic Emissions System for Photochemical Grid Modeling in California. Final Report. Sacramento, California Air Resources Board.

Winner, D. A. and G. R. Cass (1999). "Modeling the long-term frequency distribution of regional ozone concentrations.” Atmos. Enviro. 33(3): 431-451.

Winner, D. A. and G. R. Cass (2001). "Modeling the long-term frequency distribution of regional ozone concentrations using synthetic meteorology.” Environ. Sci. \& Technol. 35(18): 3718-3726.

Winstanley, D., R. Lackey, et al. (1998). “Acid Rain: Science and Policy Making.” Environ. Sci. \& Policy: 51-57.

WSPA (1998). WSPA Responses to the Subcommittee's Request for Information, Western States Petroleum Association.

WSPA (2001). Annual Report. Glendale, Western States Petroleum Association.

WSPA. (1996). Draft letter to BAAQMD requesting an estimate of the population exposure effects of the refinery BARCT NOx rule, Chevron Research and Technology Company.

Wynne, B. (1988). "Unruly Technology: Practical Rules, Impractical Discourses and Public Understanding.” Social Studies of Sci. 18(February): 147-167. 
Yang, Y. J., J. G. Wilkinson, et al. (1997). "Fast, direct sensitivity analysis of multidimensional photochemical models.” Environ. Sci. \& Technol. 31: 2859-2868.

Yearley, S. (1996). Nature's Advocates: Putting Science to Work in Environmental Organizations. Misunderstanding Science? The Public Reconstruction of Science and Technology. A. I. a. B. Wynne. Cambridge, Cambridge University Press.

Yin, R. (1994). Case Study Research: Design and Methods. Thousand Oaks, SAGE Publications.

Ziman, S. (1993a). ARB Workshop on Identifying Ozone Transport Couples; Input for ARB Board hearing in August to Update Couples Regulated under Transport Mitigation Regulations, Chevron Research and Technology Company.

Ziman, S. (1993b). ARB Workshop on Transport Couples, Chevron Research and Technology Company.

Ziman, S. (1993c). Recommendation for WSPA to Request BAAQMD to Calculate Population Exposure for Impacts of NOx Regulations, Chevron Research and Technology Company.

Ziman, S. (1996a). ARB Modeling Update for Refinery NOx Rule Assessment, Chevron Research and Technology Company.

Ziman, S. (1996b). Fax attaching Alpine Geophysics study of NOx BARCT effects, Chevron Research and Technology Company.

Ziman, S. (1996c). Note to ARB regarding the ARB workshop updating their transport assessment, Chevron Research and Technology Company.

Ziman, S. (2000). Interview. February 10, 2000.

Ziman, S. (2003). Pers. Comm. March 26, 2003. 


\section{APPENDIX A: CASE STUDY METHODS AND PROCEDURES}

\section{A. Introduction}

This chapter describes how the research objectives and questions guiding this work are achieved and answered using qualitative research methods. Detailed within are the case under study and data gathering techniques. Interview respondents are described in detail, as are data analysis procedures and the limitations of the approach. The chapter ends with a description of the research products.

This chapter discusses the methods used to describe and evaluate the Central California air quality planning process (abbreviated as Cent CA AQ Planning). Data gathering and analysis techniques are those of social scientists, so they may be unfamiliar to technically trained in the natural sciences.

\section{B. Case Study Research}

This work examines the case of planning to meet ozone air quality standards in Central California during the decade of the 1990’s. Data are gathered through interviews and archival research. In addition, literature is reviewed to survey the state of model uncertainty analyses, and to use theory pertaining to planning and decision-making.

\section{The Case Study}

Yin (1994) considers case studies useful for the "how” and "what" questions asked by this research. ${ }^{123}$ Dutton and Kraemer (1985) use the case study approach as their primary data in Modeling as Negotiating, but they use a multiple-case design. This research relies on a single case. It is an intrinsic case study, as opposed to an instrumental or collective

\footnotetext{
${ }^{123}$ Research questions are presented in Chapter I - Introduction.
} 
case study, because better understanding of a particular case is sought (Stake 2000). A single case study design is appropriate when the case is a "critical” test of a theory, an “extreme or unique” case, or a “revelatory” case (Yin, 1994). ${ }^{124}$

Ozone air quality planning in Central California in the 1990's is unique in two important ways. First, the two airsheds of interest in Central California are special, but for different reasons. The San Joaquin Valley has among the Nation's most severe air quality problem, experiencing regular violations of health-based standards for ozone and particulate air pollution. Unlike any other major metropolitan area, the San Francisco Bay Area achieved compliance with the Federal ozone standard only to fall out of compliance the following year. Furthermore, the Bay Area is a source of pollution transported to the San Joaquin Valley airshed.

Second, Central California is home to inter-agency, multi-stakeholder, well-funded air quality studies. In the 1990’s, the San Joaquin Valley Air Quality Study (SJVAQS) produced a state-of-the-science modeling system and among the most robust observational databases available for an air quality modeling application.

\section{Boundaries and Outcomes}

Any case study involves two basic questions:

- What are the boundaries of the case?

- What are the outcomes of interest?

This case begins with the concurrent development of a photochemical air quality modeling system (called SARMAP ${ }^{125}$ ) and intensive field studies to provide input data

\footnotetext{
${ }^{124}$ A revelatory case is an opportunity to study a phenomena not previously observed.
} 
for a modeling application. Ultimately, SARMAP was applied to the study of ozone air quality in the SJV to support production of the federally-required 1994 San Joaquin Valley Ozone Attainment Demonstration Plan (94 Ozone Plan). Along the way, SARMAP was used for other planning efforts, notably assessment of the significance of transported emissions from the San Francisco Bay Area into the SJV. Also, SARMAP was notable for its absence in ozone planning required by the California Clean Air Act (CCAA), largely because the language of the law did not require modeling. These three planning requirements - federal ozone standards, state ozone standards, and transport assessment - delineate decision-making behaviors examined in detail in this case.

Although air quality studies in Central California and planning efforts mandated by federal and state clean air legislation are ongoing, this research is focused temporally on the 1990's. During that decade, the SJVAQS commenced and concluded, while CARB produced three transport assessment reports, and two local air quality management agencies (i.e., BAAQMD, SJVUAPCD) produced numerous air quality plans. Thus, the broad case begins with air quality studies and modeling applications. It ends with air quality control decisions codified in local planning documents.

Also of interest is the broader structure of the modeling study, which can be grouped as:

1. Setting (e.g., regulations, laws, participants)

2. Rules-of-the-Game (e.g., problem definition, method of participation, model selected)

3. Modeling Details (e.g., protocol, inputs, technical controversies).

${ }^{125}$ SARMAP stands for SJVAQS/AUSPEX Regional Modeling Adaptation Project. 
Rules of the game, technical controversies and other modeling details, and the regulatory, sociopolitical and air quality settings comprise the independent variables. The dependent variables, outcomes, are the positions taken by modeling study or planning participants regarding the technical controversies. Ultimately, these positions translate into decisions that are codified in emissions control plans.

Majone (1989, page 114) writes, "No descriptive or prescriptive policy analysis can be complete that does not explicitly take institution-changing behavior into consideration.” This research is concerned with why agency personnel or other stakeholders may resist change. That is, why scholarly suggestions and theoretical imperative for addressing, head on, the limitations of modeling studies have not been heeded.

Roth et al. (1997) provide a comprehensive list of characteristics of an air quality modeling study. ${ }^{126}$ The following controversies were present in this case, and were topics of discussion for interviews:

- Model selection

- Meteorological conditions or episodes to model

- Database assessment

- sufficiency for conducting any modeling

- sufficiency for assessing the reliability of modeling results

- sufficiency for modeling of a given episode

- Process and criteria for performance evaluations, with special consideration of the time gaps between performance testing and decision to rely on modeling results

- Estimate of biogenic VOC emissions

${ }^{126}$ Refer to Table III-B in Chapter III - PAQSM and Their Uncertainties. 
- Estimate of anthropogenic mobile source emissions

- Estimate of anthropogenic stationary source emissions

- Estimate of boundary conditions

- Definition of the modeling domain

- Decision to focus controls on VOC or $\mathrm{NO}_{\mathrm{x}}$ emissions

Outcomes of interest are planning decisions, as well as the negotiations that preceded, occurred during, or followed modeling studies. Especially relevant are the technical controversies and their resolution, as they highlight the management and treatment of uncertainties. Attention is focused on the positions taken by individuals and organizations regarding these uncertainties, and how positions were influenced by factors in addition to purely scientific considerations.

\section{Cases within the Case}

Three types of planning documents - Ozone Attainment Demonstration Plans, Clean Air Plans, and Transport Assessments - are the touchstones within the case of Central California air quality planning. Two specific decisions about the control of $\mathrm{NO}_{\mathrm{x}}$ emissions from petroleum refineries, and their associated controversies, emerged as particularly interesting. They hinged upon, or were at least justified by, modeling results from the same modeling application (i.e., SARMAP applied to simulate an episode of high ozone observed in August 1990), even though different control scenarios and output metrics were considered. The players and control technologies at issue were largely the same. The outcomes - decisions to implement $\mathrm{NO}_{\mathrm{x}}$ controls - were different. 
Westside Kern County Refinery $\mathrm{NO}_{x}$ Controls

The 94 Ozone Plan set forth a plan to attain the federal 1-hour ozone standard by 1999. The basis for the plan was a SARMAP modeling application to simulate an ozone event observed in early August 1990. The plan called for the implementation of numerous controls on both $\mathrm{NO}_{\mathrm{x}}$ and VOC emissions, and relied on increasingly stringent vehicle emissions standards. Just prior to completion of the plan, a final emissions scenario was modeled that omitted $\mathrm{NO}_{\mathrm{x}}$ controls in the western side of Kern County. The model showed that the region would attain the standard by 1999 without the $\mathrm{NO}_{\mathrm{x}}$ controls, so the plan did not include them.

\section{San Francisco Bay Area Refinery $\mathrm{NO}_{x}$ Controls}

In the early 1990's the Bay Area Air Quality Management District began promulgating rules to require $\mathrm{NO}_{\mathrm{x}}$ emissions controls on petroleum refineries in the S.F. Bay Area. The rationale and legal impetus for these controls was the role of $\mathrm{NO}_{\mathrm{x}}$ in tropospheric ozone formation both within the Bay Area and downwind in the SJV.

Early modeling studies by the BAAQMD brought into question the downwind benefits of $\mathrm{NO}_{\mathrm{x}}$ controls, suggesting instead that $\mathrm{NO}_{\mathrm{x}}$ controls might worsen local air quality. ${ }^{127}$ Eventually, SARMAP modeling was applied to evaluate the benefits of $\mathrm{NO}_{\mathrm{x}}$ refinery controls. Although SARMAP results corroborated early studies, the $\mathrm{NO}_{\mathrm{x}}$ controls were required by the BAAQMD.

\footnotetext{
${ }^{127}$ Under the right atmospheric conditions, nitrogen oxides emitted into the atmosphere may react with available ozone to form nitrogen dioxide and diatomic oxygen, thereby decreasing the ozone concentration. For a detailed explanation of this chemistry, refer to Chapter X.
} 


\section{Data Sources}

Data were gathered from interviews with air quality planners and modelers, public officials and agency personnel, other technical experts, and stakeholders, as well as archival research. These qualitative data - written and spoken words - are "a source of well-grounded, rich descriptions and explanations of processes occurring in local contexts” (Miles and Huberman 1994, page 15). Yin (1994) describes interviews as “essential sources of case study information.” Although archival review provided the background details necessary to script interview questions, both the archives and interviews were the primary data used for analysis.

\section{Archival Research}

Archives review preceded interviews and continued throughout the research to provided corroborative evidence with which to evaluate respondent accuracy, honesty and biases. Where possible, archival materials were cited and quoted preferentially over respondents’ statements.

One key source of information was Dr. Steven Ziman . He granted access to his extensive archives, which proved essential once the official SJVAQS records were lost from the CARB library. Ziman's archives were valuable, but other documents were reviewed to avoid potential bias. Archival sources include:

- Public agency and private consulting reports

- Public meeting transcripts

- Personal correspondences, including letters, memorandums and e-mails

- Legal transcripts, discovery documents, and court opinions 
- Laws and regulations

- Journal articles and books primarily about air quality engineering, planning, and science and technology studies

Key documents include:

- 1994 San Joaquin Valley Ozone Attainment Demonstration Plan (94 Ozone Plan)

- 1999 and 2001 Bay Area Ozone Attainment Demonstration Plan (99 and 01 Ozone Plans)

- 1990, 1993, 1996 Transport Assessments by CARB

- SJVAQS Policy-Relevant Findings

\section{Interview Procedures}

\section{Logistics}

Unstructured interviews were conducted in privacy as a one-on-one conversations. ${ }^{128}$

In keeping with the requirements of the University of California Committee on Human

Subjects (U.C. Berkeley project number 99-8-118), at the outset of the interview each

respondent was asked to acknowledge verbally that they are aware of the following:

- The interviewer is James Fine, a graduate student at U.C. Berkeley in the Energy and Resources Group, as well as a researcher at Lawrence Berkeley National Laboratory.

- They are part James Fine’s dissertation research

\footnotetext{
${ }^{128}$ Bernard (1995) discerns four types of interviews according to the extent of researcher control: informal - lacking any structure or control, the informal interview involves the researcher making notes about overheard or casual conversations without a formal interview event. unstructured - also called "open-ended”, the unstructured interview exerts "minimum control over the informant's responses” but involves a formal interview event and has a clear plan. semi-structured - the semi-structured interview requires a list of questions and topics that are addressed in a specific order.

structured - presenting each respondent with an "identical set of stimuli", the structured interview may be a survey with an explicit set of instructions to guide the interviewer.
} 
- Their participation offers neither risks, nor direct benefits to them

- They can stop the interview at any time

- They know that the interview is being recorded

- They will have an opportunity to approve any statement or quotes attributed to them

All interviews were recorded "on the record.” Immediately following interviews, notes were recorded, either verbally on the tape, or in written form. Interviews were transcribed by the interviewer, during which time additional notes were made.

Per the final bullet above, each respondent quoted in the written portion of the dissertation was given both a copy of the original transcript, and a draft of the dissertation for approval and modification, as necessary.

\section{Content}

A set of questions was prepared prior to each interview. This "script" was used as a loose guide for the topics, and order of topics, to be covered in the interview. Interview topics focused on specific technical controversies, but also explored the respondents' personal history, experiences, values, and policy positions. The posing of science-based questions was necessary both to explore relevant topics and to establish interviewer credibility. In addition, the topic of uncertainty was discussed explicitly. A list of the questions from which scripts were generated is included as Appendix A. Topics for questions included the following:

- Understanding Role and Responsibilities

- Context and Perspective 
- CAP Process

- SIP Process

- Conception of Uncertainty

- Role of Models

- Use of Uncertainty Information

- Control of Modeling

- Personal Philosophy

Some respondents demonstrated an understanding of uncertainties in modeling.

Others were less conversant in expressing their knowledge. To address this concern, the

following statement was read to assure common understanding about uncertainty:

Air quality planners use models to develop estimates of expected air quality, as well as some judgment as to whether attainment would be achieved.

There are uncertainties associated with the model itself, inputs to the model, and decisions derived, at least in part, from the modeling. As a consequence, the estimates of expected air quality have uncertainties. The numbers produced by the model are uncertain and have errors associated with them. Since there are uncertainties, a range of possible outcomes can be estimated. Even though the likelihood of specific outcomes varies, they remain possibilities. The possibilities encompass both ranges that are acceptable and are not acceptable to regulatory requirements. This situation leads to a set of questions.

Rarely was every question on the list posed in a single interview. Often, questions not on the script were asked as part of the flow of conversation. The technique for presenting questions generally took two forms. There were innocuous get-to-know-you questions, such as "How long have you been with this organization?" or "Has your perception of the uncertainties in modeling changed during your work here?” Other questions were more argumentative, though none of the interviews were acrimonious. When the respondent articulated a controversial opinion an attempt was made to offer the 
opposing view to further and deepen the conversation. However, the interviewer did not necessarily claim to agree with the opposing view.

\section{Population Sampled}

In their case studies of fiscal impact analysis modeling and its use for planning, Dutton and Kraemer (1985) classify modeling study participants according to their level and type of involvement or the decisions derived from it. These groups locate on a matrix, as shown in Table APPA-A. The matrix is useful for thinking about whose views should be solicited through interviews.

Table APPA-A. Classification of Participants in the Modeling Process ${ }^{129}$

\begin{tabular}{|c|l|l|}
\hline $\begin{array}{l}\text { Nature of Primary } \\
\text { Interest in the } \\
\text { Modeling Effort }\end{array}$ & \multicolumn{1}{|c|}{ Primary Scope of Interest in the Modeling Effort } \\
\hline Political & $\begin{array}{l}\text { Macro-level } \\
\text { Partisan Groups: periodic but } \\
\text { intensive involvement in planning } \\
\text { decisions. }\end{array}$ & $\begin{array}{l}\text { Political Elites: continuous but non- } \\
\text { intensive involvement in planning } \\
\text { decisions. }\end{array}$ \\
\hline Technical & $\begin{array}{l}\text { Modeling Supporters: periodic and } \\
\text { intensive involvement in the } \\
\text { modeling study, but not necessarily } \\
\text { planning decisions. }\end{array}$ & $\begin{array}{l}\text { Policy Analysts: continuous and } \\
\text { intensive involvement in both the } \\
\text { modeling study and planning } \\
\text { decisions. }\end{array}$ \\
\hline
\end{tabular}

Most respondents had a technical interest in the modeling, although some were representatives of partisan groups. Five factors determined who was interviewed:

1. Familiarity and access: Initial interviews were conducted with those known to the researcher.

2. Snowball: respondents were asked who else I should interview.

3. Knowledge: Those with detailed knowledge of SARMAP modeling, the SJVAQS, or air quality plan production were sought out preferentially over those more oriented to the political aspects of planning and decision-making. Thus, with respect to Table APPA-A, more emphasis was placed on interviewing modeling supporters and policy analysts than partisan representatives and the political elite, who received secondary and tertiary consideration, respectively.

${ }^{129}$ Expanded from Dutton and Kraemer (1985, Table 6.1, page 140). 
4. Proximity: Respondents located in California, particularly the Bay Area or in Fresno, were given priority over others. For example, whereas it was relatively easy to interview staff at EPA Region IX offices located in San Francisco, no interviews were conducted at EPA Headquarters in Washington, D.C.

5. Representation: Having located no representatives of the environmental community in the SJV, perspectives were sought from those who were participants in Bay Area air quality planning even if they were not necessarily familiar with concurrent efforts in the SJV.

\section{Description of Individuals Interviewed}

Individuals interviewed are listed in Table APPA-B.

Many different people were engaged in the SJVAQS and plan production, but some were influential in both. Of course, thousands, even millions, of people are stakeholders in Central California air quality planning. The foci for interviews were those who participated in plan production, modeling studies in support of plans, and members of the Policy and Technical Committees overseeing air quality studies. ${ }^{130}$

The Chair of the Policy Committee changed during the SJVAQS, but remained an executive policy-maker at CARB all the while. Ms. Jan Sharpless chaired both the SJVAQS Policy Committee and CARB’s Board in the early 1990’s. She was interviewed as part of this research. Two additional members of the committee, Mr. David Howekamp and Mr. Manuel Cunha, were interviewed. Howekamp directed EPA Region IX Pacific Southwest Air Division from 1982 through 2000. As President of the Nisei Farmers League, Cunha was recommended by other respondents as one who’s views should be considered in this research. Although these individuals offered valuable and valid perspectives, they had limited familiarity with the technical details, controversies and limitations attending SARMAP and its application to the 94 Ozone Plan.

\footnotetext{
${ }^{130}$ Although there are many air quality studies, the Technical and Policy Committees are the same.
} 
Also interviewed were two Deputy Executive Officers at CARB, Mr. Michael Scheible and Ms. Lynn Terry . Although neither formally served on SJVAQS committees, Scheible participated at times on behalf of CARB. He also oversaw plan production and the Modeling and Technical Support Division during the 1990's. Terry now has those responsibilities.

Members of the Technical Committee were a higher priority than Policy Committee participants. The Technical Committee members have the best understanding of the modeling, including what can and cannot be done with the models, and what was and was not done for 1994 SARMAP. Tables II-C and II-D list committee members and show who was interviewed for this research.

In total, five of the twelve members of the Technical Committee were interviewed; a sixth, Dr. Philip Roth, was consulted but not interviewed formally. He provided valuable advice throughout this research. Of the remaining six Technical Committee members, one is deceased and two were listed as "past members" in SJVAQS documentation (SJVAQS 1996).

Some Technical Committee members were integrally involved in both model development and application. Dr. Andrew Ranzieri chaired the Technical Committee and managed CARB’s Modeling Support Division for over 25 years starting in 1975. Thus, he played a pivotal role in both the development of the SARMAP modeling system, and its application for the 94 Ozone Plan. He graciously submitted to a 90-minute interview. His supervisor, Mr. Donald McNerny, and two of his staff, Mr. John DaMassa and Dr. Saffet Tanrikulu were also interviewed. The latter two conducted SARMAP modeling. 


\section{Table APPA-B: Interview Respondents}

\begin{tabular}{|l|l|l|}
\hline Respondent & Title, Division & Organization \\
\hline Angel, Bradley & Executive Director & Greenaction \\
\hline Beardon, Katy & Supervisor, Plan and Rule Development & SJVUAPCD \\
\hline Bohnenkamp, Carol & Environmental Engineer & EPA \\
\hline Cunha, Manuel & President & Nisei Farmers League \\
\hline DaMassa, John & Supervisor, Planning and Technical Support & CARB \\
\hline DeMandel, Robin & Manager, Research and Modeling & BAAQMD \\
\hline Hayes, Scott & Attorney & Independent \\
\hline Jones, David & Planning Manager, Planning & SJVUAPCD \\
\hline Karras, Gregory & Senior Scientist & CBE \\
\hline Leong, Gene & Executive Director & ABAG \\
\hline McNerny, Donald & Supervisor, Planning and Technical Support & CARB \\
\hline Nester, Scott & Supervisor, Plan and Rule Development & SJVUAPCD \\
\hline Newell, Brent & Staff Attorney & CRPE \\
\hline Ramo, Alan & Director & GGUELJC \\
\hline Ranzieri, Andrew & Manager, Planning and Technical Support & CARB \\
\hline Roggenkamp, Jean & Manager, Planning and Transportation & BAAQMD \\
\hline Scheible, Michael & Deputy Executive Officer & CARB \\
\hline Schonbrunn, David & Director & TransDef \\
\hline Sharpless, Jan & Chairwomen (formerly) & CARB \\
\hline Shipp, Evan & Supervisor, Air Quality Analysis Section & SJVUAPCD \\
\hline Sweet, James & Modeler, Air Quality Analysis & SJVUAPCD \\
\hline Tanrikulu, Saffet & Modeler, Planning and Technical Support & CARB \\
\hline Terry, Lynn & Deputy Executive Officer & CARB \\
\hline Umeda, Thomas & Modeler & BAAQMD \\
\hline Wang, Michael & Manager of Operations and Environmental Issues & WSPA \\
\hline Weisser, Victor & President & CCEEB \\
\hline Ziman, Steven & $\begin{array}{l}\text { Senior Staff Scientist, } \\
\text { Air Issues and Technology }\end{array}$ & Chevron-Texaco \\
\hline
\end{tabular}

BAAQMD = Bay Area Air Quality Management District

$\mathrm{CARB}=$ California Air Resources Board

$\mathrm{CBE}=$ Citizens for a Better Environment

CCEEB = California Council for Environmental and Economic Balance

CRPE = Center on Race, Poverty and the Environment

GGUELJC = Golden Gate University Environmental Law and Justice Clinic

SJVUAPCD = San Joaquin Valley Unified Air Pollution Control District

TransDef $=$ Transportation Solutions Defense and Education Fund

WSPA $=$ Western States Petroleum Association 
Table APPA-C: SJVAQS Policy Committee

\begin{tabular}{|c|c|c|}
\hline Name & Organization & Interviewed? \\
\hline $\begin{array}{l}\text { Doug Vagim } \\
(\text { Chair, 1995-...) }\end{array}$ & CARB & No \\
\hline $\begin{array}{l}\text { Jaqueline Schafer } \\
\text { (Chair, 1993-1994) }\end{array}$ & CARB & No \\
\hline $\begin{array}{l}\text { Jananne Sharpless } \\
\text { (Chair, 1985-1993) }\end{array}$ & CARB & Yes \\
\hline $\begin{array}{l}\text { Gordon Duffy } \\
\text { (Chair, 1983-1985) }\end{array}$ & CARB & No \\
\hline Pauline Larwood (past member) & Kern County Supervisor & No \\
\hline May Kay Shell & Kern County Supervisor & No \\
\hline Judy Andreen (past member) & Fresno County Supervisor & No \\
\hline Dave Howekamp & EPA Region IX & Yes \\
\hline Phil Brady & U.S. Department of Defense & No \\
\hline Brenda Mohn & U.S. Department of Defense & No \\
\hline Les Clark & Independent Oil Producers Agency & No \\
\hline Bill Brommelsiek & Chevron U.S.A. & No \\
\hline Catherine Reheis & WSPA & No \\
\hline Dave Crow & SJVUAPCD & No \\
\hline Bill Harper & Shell Oil (CalResources) & No \\
\hline John Torrens & Pacific Gas \& Electric & No \\
\hline Manuel Cunha & Nisei Farmers League & Yes \\
\hline Peter Mueller & Electric Power Research Institute & No \\
\hline Jon Kennedy & U.S. Forest Service & No \\
\hline Jan Bush & BAAQMD & No \\
\hline Tom Nichols & National Park Service & No \\
\hline Jack Lagarias & CARB Board Member & No \\
\hline Valerie Nera & State Chamber of Commerce & No \\
\hline Kennan Beard & Beard Land Development & No \\
\hline Merlin Fagan & California Farm Bureau & No \\
\hline Donald Gordon & Agricultural Council of California & No \\
\hline Gordon Kennedy & Private Citizen & No \\
\hline James Boyd & CARB & No \\
\hline
\end{tabular}


Table APPA-D: SJVAQS Technical Advisory Committee

\begin{tabular}{|l|l|c|}
\hline Name & Organization & Interviewed? \\
\hline $\begin{array}{l}\text { Andrew Ranzieri } \\
\text { (Chair) }\end{array}$ & CARB & Yes \\
\hline Dick Thuillier & Pacific Gas \& Electric Company & $\begin{array}{c}\text { No } \\
\text { (deceased) }\end{array}$ \\
\hline Paul Solomon & Pacific Gas \& Electric Company & No \\
\hline Steven Ziman & Chevron U.S.A. & Yes \\
\hline Alan Hansen & Electric Power Research Institute & No \\
\hline Carol Bohnenkamp & EPA Region IX & Yes \\
\hline Robin DeMandel & BAAQMD & Yes \\
\hline James Sweet & SJVUAPCD & Yes \\
\hline $\begin{array}{l}\text { Linda Chester } \\
\text { (former member) }\end{array}$ & Kern County APCD & No \\
\hline $\begin{array}{l}\text { John Vimont } \\
\text { former member) }\end{array}$ & EPA & $\begin{array}{c}\text { No, } \\
\text { but consulted }\end{array}$ \\
\hline $\begin{array}{l}\text { Philip Roth } \\
\text { (Principal Investigator) }\end{array}$ & Envair & No \\
\hline $\begin{array}{l}\text { John Watson } \\
\text { (Principal Investigator) }\end{array}$ & Desert Research Institute & \\
\hline
\end{tabular}

To compliment comments by Howekamp, Dr. Carol Bohnenkamp, EPA Region IX, was also interviewed. She served on the Technical Committee, and has reviewed modeling studies on behalf of EPA for over ten years.

The Western States Petroleum Association (WSPA) is an industry group actively involved in Central California air quality planning. WSPA provides funding for air quality studies in the SJV, has representatives on the Policy and Technical Committees, and concurrently pursues the interests of its constituency through lobbying and the production of independent research. In addition to Dr. Steven Ziman , a Chevron-Texaco scientist working closely with WSPA and a member of the Technical Committee, the Manager of Operations and Environmental Issues, Mr. Michael Wang, was interviewed.

The five staff at the SJVUAPCD interviewed for this research have combined 41 years of experience at the SJVUAPCD, averaging over eight years each. The average is 
startling when juxtaposed with the 10-year history of the air district. Three of the respondents have been there since its formation on May 25, 1992, though some worked at air quality management districts in the SJV prior the SJVUPACD formation. The planner who supervised production of the 94 Ozone Plan, Mrs. Katherine Beardon, is retired, but nonetheless agreed to be interviewed. After her, Mr. Scott Nester became supervisor of plan production; he was also interviewed.

Others interviewed offered perspectives developed from a long history of work on California air quality. Leong, Roth, DeMandel, Ziman , Ranzieri, and McNerny started working on it in the 1970's, or earlier.

Modelers and planners at the BAAQMD were also interviewed. Two interviews were conducted with Dr. Robin DeMandel, who has been at the BAAQMD since 1973, and has managed research and modeling there since 1988. Also interviewed were Ms. Jean Roggenkamp, Manager of Planning and Transportation, and Mr. Thomas Umeda, a modeler with 20 years experience at the BAAQMD.

Environmental representatives interviewed included Mr. Bradley Angel, Executive Director of Greenaction, Mr. Alan Ramo, J.D., Golden Gate University Environmental Law and Justice Clinic and former legal director for Citizens for a Better Environment, and Mr. David Schonbrunn, Director of the Transportation Solutions Defense \& Education Fund (Transdef). Two short telephone interviews were conducted with Mr. Greg Karras, Senior Scientist at Citizens for a Better Environment, and Mr. George Hayes, J.D., an attorney working with Our Children’s Future, amongst others. The environmentalist respondents were identified through their active participation in Bay 
Area air quality planning, particularly regarding the recent Ozone Attainment Demonstration Plan adopted in 2001 (01 Ozone Plan) (BAAQMD 2001a). Although familiar with air quality planning in the Bay Area, few were familiar with the details of the SARMAP modeling or the 1994 Ozone Attainment Plan for the SJV.

In addition to pursuing members of the Policy and Technical Committees and influential public agents, selection of interviews followed a snowball approach. That is, one respondent recommended another, and so on. In some respects, it would have been desirable to interview more people. However, practicality prevailed when interviews began yielding decreasing returns. Although each encounter was beneficial, they were confirming what had already been learned, rather than providing new insights.

In total, thirty interviews were conducted with twenty-seven people. The number of respondents at each organization is shown schematically in Figure APPA-1. Interviews averaged over one hour in length and transcripts were typically 30 pages of doublespaced text. 
Figure APPA-1: Number of Respondents at Each Organization

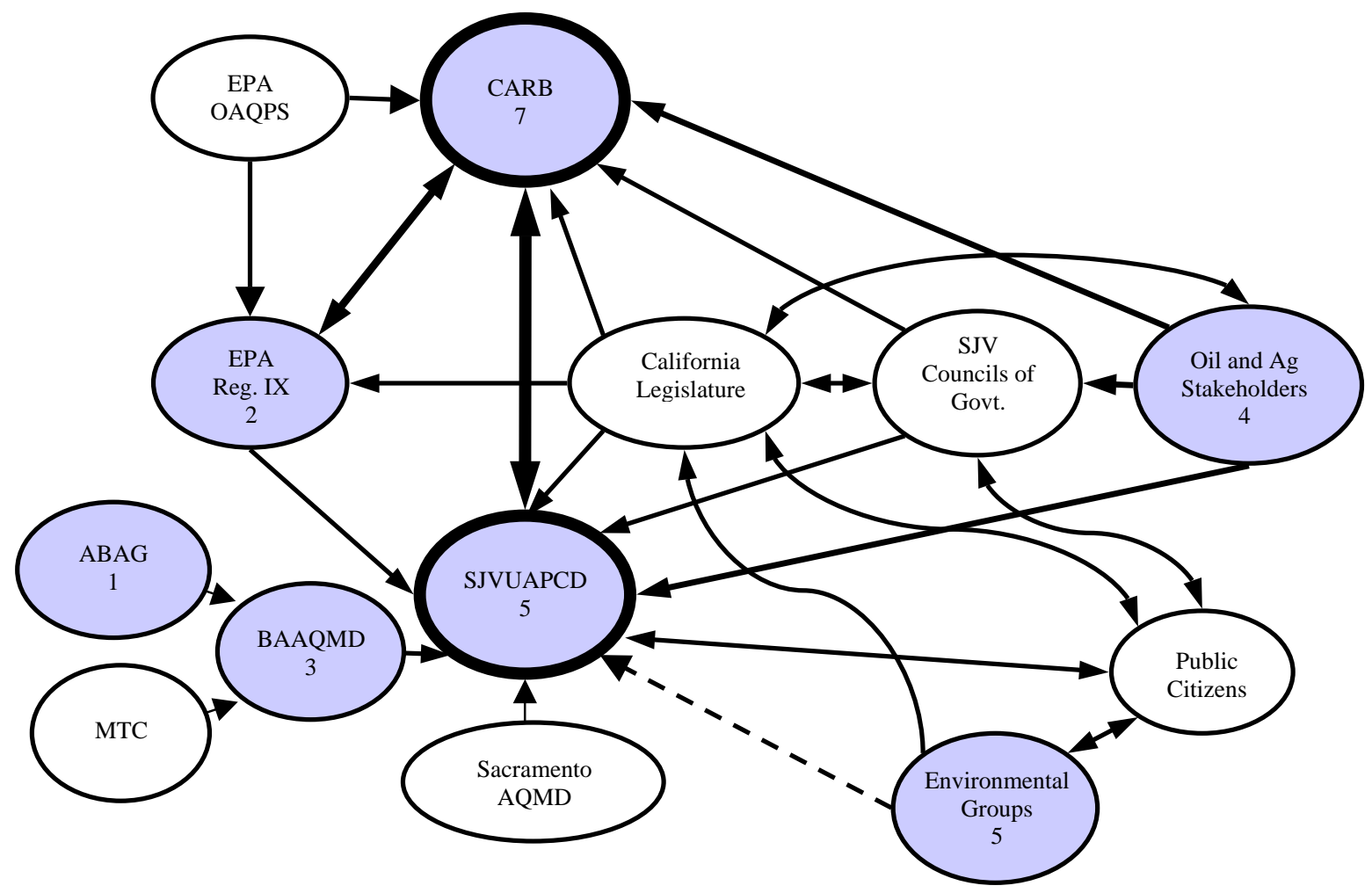

3. Limitations of Interviews

\section{Undocumented and Preexisting Relationships}

Many people sitting on SJVAQS committees were active in Central California air quality planning long before the commencement of the studies. Relationships and policy positions were well established prior to the start date of this case study. Players have historical and geographic networks that extend far beyond the 94 Ozone Plan network shown in Figure IV-6 of Chapter VII. For example, Leong, Director of ABAG, is a long-time friend of Ziman, Chevron-Texaco. They where undergraduate chemistry lab partners at the University of Michigan. Roth, Co-Principal Investigator of the SJVAQS Technical Advisory Committee, was a principle at Systems Applications, Inc. in the early 1970’s when Leong worked there. 
Similarly, DeMandel, Director of modeling at the BAAQMD, often carpooled to meetings in Sacramento with Ziman and Roth. These three men came to be viewed by some as having similar ideas about modeling and the to study uncertainty. They have been regularly united in their disagreements with technical and executive personnel at CARB. Thus, entrenched positions and scars from previous disagreements are important, yet hard to see, parts of the air quality planning network observed in this case study.

In addition to preexisting relationships, numerous relationships of potential importance remain undocumented by this research. For example, there is a direct connection between local government officials and board membership at local planning agencies, such as the BAAQMD and the SJVUAPCD. Oftentimes, they are the same people. No board members or local government officials were interviewed for this research. Although their perspectives are less relevant than those with detailed knowledge of the technical issues and the planning process, their interactions remain a significant, yet largely unknown, aspect of the networks studied. For the purposes of this study, however, it is sufficient to acknowledge unexplored, possibly unknowable, linkages, and their importance relative to other linkages with respect to the production and use of modeling.

\section{Representativeness}

As shown in Figure APPA-1, some organizations were emphasized over others. The diagram does not show decision-making authorities or responsibilities. Agents at EPA headquarters in Research Triangle Park and Washington, D.C., were omitted, not because their views are irrelevant, but because they are so far away. Eventually, they will be made aware of this research, and they will be consulted informally. The omission is 
justifiable, since EPA policy positions were well represented by the other respondents, notably EPA agents working in the Region IX office.

Nobody who does emissions inventory development was interviewed for this research. Finding that most respondents blame the emissions inventory as the major source of uncertainty may just be "passing the buck.” However, in another example of using the printed text to ground findings, the literature agrees that emissions estimates are the major source of uncertainty attending air quality simulation models.

Some interviews were sought, but never conducted. Most notable is Katherine Reheis-Boyd, who represents WSPA on the Policy Committee. Many respondents recommended her as having an important perspective. Unfortunately, she was not available to be interviewed. Division directors at CARB and local air districts were not interviewed, even though they may have offered a broader view of the air quality planning and research processes.

Last, no organized environmental coalitions were participants in the planning and research processes studied. To avoid total omission of environmental representation, interviews were conducted with environment activists who engaged in Bay Area ozone planning through written comments and public testimony.

\section{Sample Size}

Sample size matters when you want to describe, summarize and analyze data using statistical methods. In such cases, the total population and desired level of confidence interplay to determine the needed size of the sample. Furthermore, it would be 
paramount that samples be selected at random. Interview selection was deliberate rather than random.

Data are used to generate non-statistical statements and findings about the case. For example, narratives are identified, and attributed to individuals or organizations, where the data support doing so. Looking at Figure APPA-1, it is reasonable to question how, for example, a narrative may be assigned to the CARB Executive Office based on interviews with only two individuals there. Although valid, the question is easily answered. Conclusions are based on far more than two conversations, as that is not the correct way to count the data. The narratives and other stories are understood and described by many of the respondents. Narrative associations are based on comments made by all of the respondents, not solely those for whom a specific narrative summarizes their policy view. When the data are viewed in light of their ability to describe other respondents, a few interviews have the potential to provide an extensive, self-checking, web of evidence. Furthermore, conclusions are supported and corroborated with archival materials in print.

In reality, respondents did not demonstrate consistent narratives, but often invoked more than one narrative during the course of the interview. A staff person at EPA Region IX may say something about EPA. Their statement may be representative of EPA policy if he speaks with the right voice. Nonetheless, conclusions are also dependent on what WSPA representatives or air district staff say about EPA. The result is a complex weave of claim and counter claim, story and nonstory, that makes interviews so rewarding and offers a product that is more than the sum of its parts. 
The clarity and defensibility of conclusions about agency policy also depends, in part, on the voice used by respondents. The literary conception of voice is invoked in this case. For whom is the respondent speaking? Is the person speaking as a voice of one, and just one opinion, or as a loudspeaker transmitting agency policy without interpretation? Where the latter is true, as is most common and, in lieu of other evidence, the default assumption, really only one respondent is necessary to delineate agency policy. In fact, no respondent need label an organization if there are ample printed data.

\section{Data Analyses}

Miles and Huberman (1994), amongst others, note the lack of formal, canonized qualitative data analysis methods and courageously admit that in the interest of doing "good analysis", it is the "creation, testing and revision of simple, practical, and effective analysis methods" that remains an ongoing priority for qualitative researchers . Their acknowledgement provided a sort of cathartic justification for the analysis approach developed for this research. Rather than following a specific set of tasks prescribed by methodologists, my methods evolved out of necessity, albeit informed from the literature about qualitative analysis methods and review of the products of the application of those methods. Thanks to the literature, analytic pitfalls have been avoided by emphasizing some methods over others.

Techniques employed are intended to be simple, transparent, verifiable, and replicable, while accepting and avoiding the challenges of qualitative research, such as allowing researcher bias to influence the interpretation of data. The analysis of words will never reveal the one social reality in need of discovery since no single reality exists. Different stories might be told with the same data. 
Concerns about bias introduced by the researcher, through data gathering and analysis, pertain to transparency and reproducibility. Those criteria are satisfied in three ways in this research. First, the methods used are simple enough to be repeated by another. Second, it is not enough to claim that the methods are simple; details presented in this chapter are intended to be sufficient for another researcher to actually use them in the same way. Third, should a researcher use these methods in pursuit of the same questions, they would arrive at the same conclusions. This last assertion cannot be proven, except to say that iterations with the data produced initial findings that were confirmed subsequently. The interpretations of the data and resulting conclusions were internally consistent. Through an iterative approach, the methods "reproduced" consistent results.

\section{Interview Transcription and Coding}

Recorded interviews were transcribed verbatim. Copious notes preceded and followed transcription. Once transcribed, interviews were coded. For this study, coding meant that respondent statements were labeled according to topics of interest. Coding categories, called nodes in the Nvivo Nudist software used for the purpose, are listed in Table APPA-F. Subcategories, or subnodes, are attributes that give detail to categories. No attributes were assigned to self-explanatory categories or to categories that would have been overly constrained had they been given attributes. For example, constraints were a recurring theme during discussion, so no subcategories were generated to avoid artificial categorization of the topic. Ultimately, however, planning constraints were synthesized during analysis and conclusion drawing into four issues: time, political will, legal requirements and scientific information. The attributes grew out of the discussion 
even though no individual respondent presented four discrete issues pertaining to

planning decision constraints.

Eleven interviews were coded in detail, whereby every sentence was labeled. Other interview transcripts were coded selectively, or simply reviewed for consistency.

Table APPA-F: Coding Nodes

\begin{tabular}{|l|l|}
\hline Category & Attribute \\
\hline Problem Definition & Regulatory Requirements Focus \\
\hline & Fear of Litigation \\
\hline & Environmental Justice \\
\hline & Transport \\
\hline & Ozone Air Quality Only \\
\hline & Extent of Needed Controls \\
\hline & Stalling to Delay Controls \\
\hline & Best Available Science Not Enough \\
\hline & Lack of Scientific Understanding \\
\hline & Incomplete Uncertainty Info \\
\hline & Growth and Land Use \\
\hline & Overall Air Quality Focused \\
\hline & Environment versus Economy \\
\hline & Political Will \\
\hline & Public Understanding \\
\hline & Politics of Uncertainty \\
\hline Uncertainty Information & Utility of Information \\
\hline & Methods for Producing \\
\hline & Extent Considered in Decisions \\
\hline & Method for Considering in Decisions \\
\hline & Types of Useful Information \\
\hline & Evolution of Perceptions \\
\hline & Information flow to Decision-makers \\
\hline & Significance of Uncertainty \\
\hline Modeling Reference & Purpose of SARMAP \\
\hline & Performance Evaluation \\
\hline & Role in SIPs \\
\hline & Capabilities \\
\hline & Role in Transport Assessment \\
\hline & Emissions Inventory \\
\hline & Input Quantities \\
\hline & Grid Resolution \\
\hline & Boundary Conditions \\
\hline & Growth Projections \\
\hline & Model Formulation \\
\hline & Compensating Error \\
\hline & Numerical Solution Techniques \\
\hline & Meteorological Model \\
\hline & Chemical Mechanism \\
\hline & \\
\hline & \\
\hline & \\
\hline &
\end{tabular}




\begin{tabular}{|l|l|}
\hline Category & Attribute \\
\hline & Implementation of Controls \\
\hline & Measurements \\
\hline Narratives & Representativeness of Episode \\
\hline & Science First \\
\hline & Efficiency and Cost-Benefit \\
\hline & Control Everything \\
\hline & Mental Maps \\
\hline & Control All Feasible \\
\hline Participation & Roth's Perspective \\
\hline & Industry Input and Funding \\
\hline & Environmental \& Community Advocates \\
\hline & EPA Opinion \\
\hline & Judicial Involvement \\
\hline 1994 Ozone Plan & Assoc. of Govt. Role \\
\hline & Decision to “Bump Up” \\
\hline & Treatment of Uncertainty \\
\hline & Federalization of Controls \\
\hline & Expectations for SJV's 2002 Ozone Plan \\
\hline & Prescriptive Yet Uncertain \\
\hline & Paper Trail \\
\hline Decisions & Politics \\
\hline & Plan Approval by ARB \\
\hline & Use of Uncertainty Information \\
\hline & Plan Approval by EPA \\
\hline CCOS & Authorities \\
\hline & Use in Draft 2002 Ozone Plan \\
\hline Constraints & Designing for Uncertainty Info \\
\hline VOCs vs NOx Control Debate & Utility of Results \\
\hline Responsibilities & \\
\hline Learning & \\
\hline & \\
\hline & \\
\hline & \\
\hline & \\
\hline & \\
\hline & \\
\hline & \\
\hline & \\
\hline & \\
\hline & \\
\hline &
\end{tabular}

Detailed coding yielded a database of textual statements that was easily sorted and searched. It rendered trivial the task of listing all respondent statements pertaining to a given category or attribute.

Categories and attributes map, roughly, to discussion topics, whereas attributes describe each category. The categories were selected by the research and, really, were used strictly for organizational purposes. It is not the categories, but their attributes and, where appropriate, attributes' dimensions that provides the evidence with which to distinguish narratives or to identify points of common agreement. Individual respondent 
statements regarding a specific attribute may vary along a defined dimension. It is this variation that provides clues to differences (or similarities where there is no variation) of opinion and, eventually, delineates narratives. These variations taken in aggregate delineate narratives. Attribute dimensions are listed in Table APPA-G.

\section{Table APPA-G: Attributes and Their Dimensions}

\begin{tabular}{|l|l|}
\hline Category/Attribute & Dimensions \\
\hline Constraints & $\begin{array}{l}\text { None } \leftrightarrow \text { time } \\
\text { None } \leftrightarrow \text { feasible controls } \\
\text { None } \leftrightarrow \text { political will } \\
\text { None } \leftrightarrow \text { information }\end{array}$ \\
\hline Need to Control NOx & None $\leftrightarrow$ max feasible \\
\hline $\begin{array}{l}\text { Need to Control } \\
\text { VOCs }\end{array}$ & None $\leftrightarrow$ max feasible \\
\hline $\begin{array}{l}\text { Benefits of dual } \\
\text { control }\end{array}$ & Best approach $\leftrightarrow$ worst possible option \\
\hline $\begin{array}{l}\text { Utility of NOx/VOCs } \\
\text { debate }\end{array}$ & Pointless $\leftrightarrow$ essential and legally required \\
\hline $\begin{array}{l}\text { Role of Models in } \\
\text { Plans }\end{array}$ & $\begin{array}{l}\text { None } \leftrightarrow \text { provide directional indication } \leftrightarrow \text { precise control strategy } \\
\text { Nevelopment } \\
\text { None } \leftrightarrow \text { specify reduction goals per regulatory requirements } \\
\text { None } \leftrightarrow \text { understand the science }\end{array}$ \\
\hline $\begin{array}{l}\text { Model } \\
\text { accuracy/precision } \\
\text { soo uncertain to be basis of plans } \leftrightarrow \text { Adequate to define for precise control }\end{array}$ \\
\hline Model Uncertainty & $\begin{array}{l}\text { Insignificant } \leftrightarrow \text { significant and important } \leftrightarrow \text { huge, too big to provide basis } \\
\text { for plans }\end{array}$ \\
\hline $\begin{array}{l}\text { None } \leftrightarrow \text { essential for meeting social goals at least cost } \\
\text { Information }\end{array}$ & $\begin{array}{l}\text { Find least cost path to attain standards } \leftrightarrow \text { control all emissions feasible } \leftrightarrow \\
\text { eliminate all emissions } \\
\text { and Mission }\end{array}$ \\
\hline $\begin{array}{l}\text { Mnthod to Manage } \\
\text { Uncertainties }\end{array}$ & Internalize and be conservative $\leftrightarrow$ Treat rigorously and explicitly \\
\hline
\end{tabular}




\section{Quotation}

Interviews and archives are quoted verbatim except where modified slightly for clarity. All added or modified text is noted with brackets. All quotes have been approved by respondents. Respondents also reviewed and commented on descriptive chapters (Chapters IX - XI), thereby providing respondent validation. The descriptive chapters rely on quotes to exemplify arguments. Ultimately, a very small percentage of interview transcripts and archival text is presented in the discussion. Conclusions derive from the weight of the data, not just the exemplary words offered for consideration.

\section{The Narrative Approach}

No hypothetico-deductive approach is employed to avoid finding in the data that which was hypothesized might be present. This approach allows for theoretical sensitivity during data analysis. For this research, it meant allowing the categories and attributes to present themselves. The interviews and subsequent coding were sufficiently flexible to allow for unanticipated issues and concerns. This is one advantage of unstructured interviews. If too structured, the respondent may be manipulated and their responses truncated, misunderstood, or biased.

Categories and attributes were not selected prior to data gathering. Of course, preconceived ideas informed the definition of the study, research questions, and objectives. Prior to embarking on interviews, a list of possible issues with potential descriptive value regarding the case under study was generated to provide a starting point examining data. The list is included as Appendix AppB-B. The list was not consulted, however, during data analysis. 
Although general topics of discussion were chosen, and those topics evolved as more was learned with each interview, coding categories and attributes emerged as a part of data analysis. For example, when discussing the topic of planning constraints, people talked about time. Time was coded as an attribute of planning constraints; its perceived importance as a constraint in decision-making is its implicit dimension.

\section{Sources of Bias in Data Analysis}

An important motivation for this research is the concern that "regulatory science" does not proceed with the same rigor, patience or methods as purer research science (Jasanoff 1996b). While it is not necessary to complicate this chapter with a digression on the "science wars", it would be negligent to claim an ability to devolve fact from rhetoric. Doing so involves choosing the rhetoric with which to forward arguments. Writing and analysis must ultimately conclude something. In so doing, some value system will be asserted; a system motivated, biased, corrupted and cured by the same scientific ideals not always upheld in air quality planning. Conclusions will be supported by the data chosen. Interpretation always contains some element of subjectivity.

It is the responsibility of the analyst to acknowledge that they may be a source of bias in the interpretation of the data. For example, coding categories, attributes and dimensions were selected by the researcher, albeit as a result of their "emergence" from the data.

Interviews were unstructured, but did build from a common set of questions. Without a systematic and, to the extent feasible, identical presentation of stimuli to respondents, it is inappropriate to count responses. Of course, many common questions were posed to 
each respondent. Still, the discussion building up to the question was not consistent. Answers might be influenced by what had already been discussed.

As a researcher with objectives that included a long list of questions, certain statements were sought. Once the respondent made a statement or mentioned a word, it triggered follow-up questions. For example, respondents were always given the chance to mention the word "politics". Once mentioned, they were asked to define it. But the same statements did not necessarily trigger consistent follow-up questions. It is not possible to know how this biased answers to common questions motivated by different discussions.

Ultimately, the narrative approach described above, and detailed throughout, was used to reduce the prospects for and impacts of researcher bias.

\section{E. Research Products}

Rosaldo (1992) defines three components of data analysis: data reduction, data display, and conclusion with verification. Data analysis involves proceeding concurrently with each component. In fact, data reduction begins prior to data gathering with, for examples, the choice of research questions and data collection techniques. The research products described below, therefore, are the results of data reduction and display and comprise an essential part of findings upon which conclusions are based and verified. Narratives delineate the arguments and the use of information, networks indicate the importance of players, as well as the sources and flows of information. Points of common agreement are derived not so much by synthesizing stories. Rather, references to constraints pertaining the planning process and the uses of models and uncertainty 
information were coded for identification. They were then sorted for summary and reporting. Interpretations and responses to the points of agreement lead to the disagreements and, in turn, the narratives. These points of agreement also corroborate the narratives to assure that they remain consistent even in agreement.

\section{Narratives Analysis}

According to Roe (1994, page 2),

[T] he stories commonly used in describing and analyzing policy issues are a force in themselves, and must be considered explicitly in assessing policy options. Furthermore, these stories often resist change or modification even in the presence of contradicting empirical data, because they continue to underwrite and stabilize the assumptions for decision making in the face of high uncertainty, complexity, and polarization.

Roe calls these stories "policy narratives”. Here they are referred to simply as narratives, but "discourses" is another appropriate term used in the literature. Hajer (1993, page 45) defines a discourse coalition as,

[A] group of actors who share a social construct. It is the ensemble of a set of story lines, the actors that utter these story lines, and the practices that conform to these story lines, all organized around a discourse.

Narrative analysis has been described as an essential method to understand "interactions of ideas, events, and institutions" and the "feelings and intentions of social actors” (Rosaldo 1992, page 127). The former, historical understanding, is an objective of this research, as it endeavors to describe air quality research and planning processes during a decade through its players (i.e., social actors), their ideas, and institutions. The latter, human agency, is also relevant since the research questions delve into how and why people and institutions used modeling as they did. 
The distillation of narratives may involved a range of formality involving the a priori definition of reference codes, syntax, grammar or forms (Manning and Cullum-Swan 1998, page 248). For this research, narratives are simply the aggregates of stories told by social actors. Rather than focusing on an individual's story, the broader stories evident through the telling and retelling of events or interpretations of events "often shape, rather than simply reflect, human conduct” (Rosaldo 1992, page 129). It would be more appropriate to refer to the narratives told in this case as meta-stories, but that term reads as less serious.

The objective of narrative policy analysis is to identify meta-narratives, based on the stories, nonstories, and counterstories (Roe 1994). Whereas some researchers focus on a story's beginning, middle and end, as well as nonstories lacking in each of those parts, this work is more concerned with how different bits of evidence inform, frame and justify stories and nonstories. To that end, the stories are more liked arguments because they have premises and conclusions (Roe 1994, page 3).

Of particular interest in the arguments forward are results of modeling studies, when offered as evidence. Of course, the extent to which a story is complete remains relevant; but of greater interest is whether or not it is informed by and consistent with available evidence. Moreover, it is the omission of reliable evidence that makes a narrative all the more distinctive and interesting. Delineation and subsequent assessment of narratives goes one step further than the evidence to the quality of that evidence. That is, the consideration of the reliability of modeling results, including the completeness with which the uncertainties are understood, characterized, considered and communicated in each narrative. 
Others studying the use of modeling in decision-making use a similar approach. Dutton and Kraemer (1985, page 140) wrote:

Any individual or group may be characterized by a mix of these interests, and, consequently, our analysis will focus on the dominant interests in order to make sharper distinctions among the participants.

Roe’s approach in Narrative Policy Analysis (1994, page 4) has an objective "to recast the issue in such a way as to make it more amenable to decision making and policymaking.” The objective of this research is to understand, through narratives and other evidence, the air quality process, notably the use of modeling results. The impetus is not to "recast the issue", rather it is to document a process as understood by its participants. In so doing, however, the issues are hopefully recast as more amenable to resolution.

Two final points about narratives are in order. First, it is possible to associate narratives with institutions or individual "policy actors” (Hajer 1993). That task is taken up in the chapter describing the Central California air quality planning process. Before doing so, however, a second point is imperative. Respondents can and do articulate the many different narratives simultaneously. Furthermore, they may do so whether or not they agree with them.

\section{Network Diagrams}

Diagrammatic representation of the networks of institutions and subdivisions within institutions is another product of this research. The purpose of their production is not the formal analyses employed, for example, by those conducing actor-network studies. Rather, the network diagrams were intended to describe interactions and to provide 
intermediate devices for drawing conclusions and understanding findings from other analyses, such as the identification of narratives.

\section{Summary of Shared Opinions}

Narratives and networks emerged from analysis of the data. So too did points of common agreement and disagreement amongst respondents. By simply accounting for myriad opinions, common opinions became obvious. This was not so much a surprise as an afterthought, as it was the disagreements that motivated the research questions and provided the initial objects of focus. Nonetheless, given the challenges of distilling metastories and diagramming the interactions of institutions after the fact, the shared opinions are perhaps the most defensible product of this research.

\section{F. Appendix APPA-A: Interview Script}

Respondent:

Location/Setting:

\section{Approvals}

Please audibly acknowledge the following:

- I am James Fine, Graduate Student, UCB-ERG

- You are part my dissertation research

- Your participation offers neither risks, nor direct benefits to you

- You can stop the interview at any time

- You know that I am recording the interview

- You will have an opportunity to approve any statement or quotes that I attribute to you 


\section{Understanding Role and Responsibilities}

- What is your title?

- How long have you worked in your current position?

- Who do you supervise?

- Who do you answer to?

\section{Context and Perspective}

- What's the air quality problem in your air district?

- How does the problem in your district relate to other districts?

- What is at stake in CAP and/or SIP planning? Who is at greatest risk? What risks?

\section{SIP and CAP Process and Involvement}

- Where you involved with

1. Production of Clean Air Plans per the CA CAA? If so, what was your role?

2. Production of State Implementation Plans per the Federal CAA? If so, what was your role?

3. transport assessment and mitigation planning?

- What difficult or controversial decisions can you recall from the 1994 SJV SIP or the 1994 BA CAP?

- What support information did you use to make these decisions?

- With whom at your agency do you work most closely on clean air plans?

- What other agencies do you work with? With whom exactly do you work? What information do you exchange? 
- Do you interact with any other agencies, such as CARB, EPA, or DOT, during plan development?

- What were some of the challenges you've faced in dealing with other agencies? How were the challenges dealt with?

- How has your or your organization’s approach to clean air planning changed since the early 1990's? What was the impetus for change? Have changes been gradual or official?

\section{Role of and Control of Modeling and Planning}

- Could you please describe the role that SARMAP modeling played in the 1994 SJV SIP development?

- Who determined the SAQM model was performing adequately for use in the 1994 SJV SIP? Was this determination ever made official?

- Of all of the agencies and individuals involved in modeling and planning for the 1994 SJV SIP, who exerted the most control on the modeling results? One the SIP? Why? Did their influence raise any concerns in your mind?

- Did politics enter into any of the planning efforts we've discussed? If so, how?

- What would be your definition of the politics associated with clean air planning?

- Do you feel the influence of politics in your work? If so, how?

\section{Conception of Uncertainty}

- When I raise the topic of uncertainty in ozone air quality planning, what comes to mind?

- What do you think of when I use the phrase "model uncertainty"? 
- Has your perception of the uncertainties changed at all in the past decade?

Read statement: Air quality planners use models to develop estimates of expected air quality, as well as some judgment as to whether attainment would be achieved. There are uncertainties associated with the model itself, inputs to the model, and decisions derived, at least in part, from the modeling. As a consequence, the estimates of expected air quality have uncertainties. The numbers produced by the model are uncertain and have errors associated with them. Since there are uncertainties, a range of possible outcomes can be estimated. Even though the likelihood of specific outcomes varies, they remain possibilities. The possibilities encompass both ranges that are acceptable and are not acceptable to regulatory requirements. This situation leads to a set of questions.

\section{Use of Uncertainty Information}

- When the technicians give you modeling results, do you ever question its reliability?

- Do the technicians mention the uncertainties associated with their results? If so, in what context and how do they communicate the uncertainties to you?

- Is uncertainty information useful to you?

- Technicians have described a situation where those making planning decisions need a model that can “demonstrate” attainment, and that they cannot use anything else. Furthermore, they observe that the regulatory structure does not allow for uncertainty information. Is this an accurate characterization of the planning task?

- How does the model verification process work? Who’s responsible for it?

- Is model verification authority ever questioned? 
- Do you feel that the operational analyses (e.g., estimation of bias and error of predicted versus observed ozone) are sufficient to determine if the model is performing adequately for use in SIP planning?

\section{SAQM Uncertainty}

- How were SARMAP modeling uncertainties incorporated into the 1994 SJV SIP?

- In retrospect, what SARMAP model uncertainty information, if any, might have been useful for the 1994 SJV SIP?

- How was the ARB's Performance Evaluation of SARMAP used by the SJVUAPCD in preparing its 1994 SIP?

- Can you think of any uncertainty information that would help you be more comfortable with technical information, such as modeling results even if they cannot “demonstrate” attainment?

- In what ways do you convey the uncertainties associated with a CAP in the document?

Read Statement: In your work, the following questions might be of interest to you, or asked of you when doing clean air planning. I will present a question, please tell me if it is of interest to you (i.e., relevant to the tasks and decisions that are your responsibility.) Also, please tell me why or why not? Or, how I can restate or refine the question to suggest information that is more useful for your work?

1. What reductions in pollutant emissions are needed to attain the ozone and particulate air quality standards?

2. What are the inaccuracies or major uncertainties associated with modeled ambient air pollutant concentrations for various emissions reduction scenarios?

3. What evidence supports the findings of the modeling and other analyses? 
4. a) What do test statistics, such as bias and error, indicate to you about model performance?

b) What level of reliability is necessary to justify decisions based on modeling results?

c) What is the probability of not attaining standards when model simulations suggest that attainment will occur?

\section{Control of Modeling}

- Could you please describe the role that O-ZIP modeling played in the 1994 BA CAP development?

- Why was modeling conducted during preparation of the 1994 BA CAP?

- Technical folks feel that O-ZIP doesn’t really tell you much about air quality, mostly because you can get any results you want. Are you aware of this concern? If so, how might the modeling results be conveyed in a way that would be more useful to you?

- Why was modeling conducted to assess transport, including studies by CARB, BA and SAI?

- Why was modeling conducted during preparation of the 1994 SJV CAP?

- Of all of the agencies and individuals involved in CAP planning, who exerts the most control on the final plan? Why? Does their influence raise any concerns in your mind?

\section{Philosophy}

- Would you consider yourself an environmentalist? If so, why? If not, why not? 
- What is your driving philosophy in terms of the need to err on the side of too much or too little emissions reductions?

\section{Closing}

I will send you a copy of the transcript from this interview so you can check it for accuracy.

If you would like, I will also tell you about the web site we construct for this project. The interview transcript WILL NOT be located there, but materials, such as reports and analyses, derived from these interviews will be available.

- Who else do you think I should speak with?

Thank you!

\section{G. Appendix APPA-B: Initial List of Issues}

\begin{tabular}{|l|l|}
\hline Issue & Description \\
\hline Problem definition & selecting a model that biases results toward one outcome \\
\hline $\begin{array}{l}\text { Opaque or "black box" } \\
\text { decision tools }\end{array}$ & $\begin{array}{l}\text { failing to acknowledge or communicate the assumptions, simplifications and } \\
\text { limitations of modeling }\end{array}$ \\
\hline $\begin{array}{l}\text { Participants and rules } \\
\text { of participation }\end{array}$ & $\begin{array}{l}\text { excluding some stakeholders or proceeding with negotiation when there are } \\
\text { significant power imbalances }\end{array}$ \\
\hline Gaming & $\begin{array}{l}\text { acknowledging uncertainties only when doing so furthers interests, or taking } \\
\text { extreme positions to negotiate a favorable compromise }\end{array}$ \\
\hline $\begin{array}{l}\text { Epistemic } \\
\text { communities }\end{array}$ & $\begin{array}{l}\text { groups of people with shared knowledge developing social contracts and } \\
\text { norms, such as using ambiguity associated with uncertainty to develop } \\
\text { consensus or selecting performance criteria that are not scientifically } \\
\text { defensible }\end{array}$ \\
\hline Political will & $\begin{array}{l}\text { basing decisions on politically salient or population notions rather than } \\
\text { objective evidence and rationale }\end{array}$ \\
\hline $\begin{array}{l}\text { Institutional and power } \\
\text { structures and } \\
\text { struggles }\end{array}$ & $\begin{array}{l}\text { institutional structures include laws, regulations, norms, organizational } \\
\text { configuration, and decision-making procedures. Power is determined by } \\
\text { authority, access to resources and information. Issues include agencies } \\
\text { taking positions that are not scientifically defensible because they search } \\
\text { power and authority, or are trying to meet responsibilities with inadequate } \\
\text { capability and authority }\end{array}$ \\
\hline
\end{tabular}

\footnotetext{
${ }^{131}$ Majone (1989) defines institutions as laws, regulations, norms, organizations, and decision-making procedures.
} 


\section{APPENDIX B - CENTRAL CA REGULATORY SETTING}

\section{A. Introduction}

This chapter provides an overview of the regulatory setting governing air quality management in California. It surveys federal requirements, notably those established by the Federal and California Clean Air Acts and resultant planning requirements. The federal, state and local planning agencies with responsibilities under these laws are described, as are their, their authorities and interdependencies. Last, federal and state requirements for using models to support planning are compared.

\section{B. Clean Air Laws}

\section{Federal Clean Air Act}

The FCAA and CCAA both provide impetus for clean air planning in Central California. The first substantive FCAA was adopted in 1970. It established the basic framework for air quality control still in force today. In addition to providing the impetus to create the U.S. Environmental Protection Agency, the FCAA establishes the basic approach to air quality planning that relies on a system of standards and permitting, as well as assumptions about the ability to develop new technologies to mitigate emissions from both point and mobile sources of emissions. Furthermore, the FCAA requires ambient air quality standards that “.... allowing for an adequate margin of safety, are requisite to protect the public health” (Section 109 (b)(1) of the CAAA of 1990).

The FCAA expanded considerably with amendments in 1977 and 1990. The law is now long and detailed, with the following major sections: 
- Air Pollution Prevention and Control - Title I establishes NAAQS for six "criteria" air pollutants. It sets forth planning requirements for areas not meeting the standards.

- Mobile Source Emissions Standards - Title II sets explicit motor vehicle emissions standards for passenger vehicles, light duty trucks and aircraft.

- General - Title III is a potpourri dealing with issues such as emergency powers, economic impact assessment, and requirements for regular air quality modeling conferences. The citizen suit provisions gives the law its teeth.

- Acid Deposition Control - Title IV addresses acidification due to deposition of air pollutants by introducing an innovative emissions trading program.

- Permits - Title V establishes the details of the permitting process required for major stationary sources.

- Stratospheric Ozone Protection - Title VI sets forth a program for reducing use of ozone-depleting substances, such as chlorofluorocarbons.

Title I of the FCAA contains great detail about the NAAQS system of assessment and planning responsibilities. It prescribes the process for setting new standards and for planning to meet standards when they are violated. Using a nonattainment classification system, the stringency of provisions increases with the severity of ambient conditions.

Following a Federalist philosophy, Title I assigns to states the responsibility for promulgating air quality management plans and implementing emissions controls.

Title I is extensive and complex, involving numerous routes for controlling pollutant emissions. In addition to planning for attainment of the $\mathrm{O}_{3}$ NAAQS by a specified date, areas designated serious, severe or extreme must reduce total VOC emissions by 15 percent by 1996 (from a 1989 emissions baseline) and three percent annually thereafter.

Although the FCAA focuses on VOC emissions reductions, the EPA interprets the provisions of Section 182(f) pertaining to planning reductions from major stationary 
sources of VOCs to also apply to major stationary sources of $\mathrm{NO}_{\mathrm{x}}$ unless air quality benefits are greater without $\mathrm{NO}_{\mathrm{x}}$ reductions (EPA 1992b). EPA acknowledges that $\mathrm{NO}_{\mathrm{x}}$ controls might not be beneficial with respect to ozone in all instances. It allows for air districts to use photochemical modeling to determine the impact of $\mathrm{NO}_{\mathrm{x}}$ control. If modeling results do not support $\mathrm{NO}_{\mathrm{x}}$ controls, then air districts may “opt out” of Section 182 (f) provisions.

Section 169a of the FCAA establishes requirements to protect visibility in national parklands. The law treats visibility as an "air quality-related value.”132 The distinction between air quality and air quality-related values is important because regulatory requirements for attaining and maintaining air quality are very different, more explicitly defined, and in many ways more stringent, than requirements for preserving air qualityrelated values. For example, the CAA does not explicitly define what characteristics comprise air quality related values, but it does specify criteria air pollutants (discussed below), ambient air quality standards for them, and allowable impacts on their ambient concentrations from new emissions sources.

In 1999, EPA promulgated rules to prevent and reduce regional haze. The rules require every state to develop plans to achieve "natural visibility conditions” by the year 2064 through “reasonable progress targets” (EPA 1997c). The final rule leaves it to the states to define targets (EPA 1999d).

By setting vehicle emissions and fuels standards, Title II gives EPA jurisdiction over motor vehicle emissions. This makes logistical sense because vehicles cross state lines.

\footnotetext{
${ }^{132}$ Other air quality related values include flora, fauna, soil, water, archaeological and paleontological cultural resources, and odor.
} 
However, there are exceptions to EPA authority over mobile sources of emissions. States may set vehicle emissions standards at least as stringent as EPA guidelines. The California Air Resources Board sets vehicle emissions and fuel standards for California. The FCAA defers to CARB's more restrictive Low-Emission Vehicle and Clean Fuels Regulations (Sect. 244 of (1990)). CARB also works with local air districts and transportation planning agencies to develop and implement transportation control measures to reduce vehicle trips using, for examples, ride sharing programs, high occupancy vehicle lanes, and public transit.

Vehicle inspection and maintenance programs are established in the FCAA, but their implementation is decided by states. Currently, the SJV implements an enhanced Smog Check II program, whereas the Bay Area uses the original Smog Check I. The SJV wants the Bay Area to implement the enhanced program and has initiated litigation toward that end (SJVUAPCD 2002).

Split responsibilities may lead to conflicting opinions, but it eases the burden on any one agency. States have responsibility for cleaning their own air through whatever measures deemed necessary and feasible. Ultimately, EPA retains approval authority.

Title IV provides another impetus to reduce emissions of oxides of sulfur and nitrogen. The approach relies on emissions permits that can be traded amongst major point sources, notably power plant operators.

\section{California Clean Air Act}

The California Clean Air Act of 1988 also established air quality goals for ambient pollutant concentrations. Clean Air Plans are required every three years when 
observations reveal violations. Unlike the FCAA, no specific deadlines are established for meeting the standards, and there are no penalties for failing to do so.

The CCAA requires that "reasonably practicable" and "expeditious" progress be made toward meeting the state standard. The CCAA defines reasonable progress as reducing five percent of precursor emissions (i.e., both nitrogen oxides and reactive hydrocarbons) per year or, if such progress is not feasible, that "all feasible measures" be implemented in an expeditious manner (CCAA, Section 40914 (b)(2)). Both the Bay Area and the SJV pursue an "all feasible measures” strategy

A second part of the CCAA is the requirement to identify and, where necessary, mitigate transport of emissions from upwind sources. Based upon "a preponderance of available evidence”, CARB must designate upwind air basins as "overwhelming, significant, inconsequential or some combination thereof" to violations of the state ozone standard in downwind air basins (CCAA, 2001) .

\section{Criteria Pollutant Standards}

The FCAA established NAAQS for six ubiquitous air pollutants: tropospheric ozone $\mathrm{O}_{3}$, particulate matter $\left(\mathrm{PM}_{10}\right.$ for particulate ten microns in diameter or smaller), lead, carbon monoxide (CO), nitrogen dioxide and sulfur dioxide. The California Clean Air Act also sets standards for these pollutants. The Federal and California standards are shown in Tables V-A and V-B. The California standards are more stringent than the Federal standards. Of these six “criteria” air pollutants, only $\mathrm{O}_{3}, \mathrm{PM}_{10}$, and $\mathrm{CO}$ concentrations violate the NAAQS in the SJV or the Bay Area. 


\section{New Standards for Ozone and Particulate Matter}

In 1999, the EPA finalized new NAAQS for $\mathrm{O}_{3}$ and particulate matter (EPA 1997a).

Based on new data about the health effects of these pollutants, the new ozone standard is a concentration threshold of $80 \mathrm{ppb}$ that cannot be exceeded by the fourth highest daily eight-hour average over a three-year period (EPA 1997a). The new particulate standard sets two concentration thresholds for particles 2.5 microns in diameter and smaller (called “fine $P M$ ” or $\mathrm{PM}_{2.5}$ ): (1) highest weighted annual mean concentration of $50 \mu \mathrm{g} / \mathrm{m}^{3}$, and (2) highest second maximum 24-hour concentration of $150 \mu \mathrm{g} / \mathrm{m}^{3}$ (EPA 1997a). Although neither of these new standards is currently in force, they are presumed imminent. Consequently, monitoring has begun in earnest to gather observations of both 8-hour averaged ozone and $\mathrm{PM}_{2.5}$ concentrations. The EPA-defined schedule for pursing the new $\mathrm{PM}_{2.5}$ NAAQS is shown in Table $\mathrm{V}-\mathrm{C}$.

\section{Table V-C: Timeline for $\mathrm{PM}_{2.5}$ NAAQS}

\begin{tabular}{|l|c|}
\hline Actions & Deadline \\
\hline Monitoring, research and evaluation & 1998 to 2003 \\
\hline Submit State Implementation Plans to EPA & 2003 \\
\hline Attain PM NAAQS & 2005 \\
\hline Attain PM NAAQS after 5 year extension & $2010^{133}$ \\
\hline
\end{tabular}

\footnotetext{
${ }^{133}$ Two additional one-year extensions of the attainment deadlines are possible.
} 
Table V-A: Federal Air Quality Standards (SJVUAPCD)

\begin{tabular}{|c|c|c|}
\hline Pollutant & Averaging Time & Concentration \\
\hline \multirow{2}{*}{ Ozone } & 8 Hour & $0.08 \mathrm{ppm}$ \\
\hline & 1 Hour & $0.12 \mathrm{ppm}\left(235 \mu \mathrm{g} / \mathrm{m}^{3}\right)$ \\
\hline \multirow{2}{*}{ Carbon Monoxide } & 8 Hour & $9 \mathrm{ppm}\left(10 \mathrm{mg} / \mathrm{m}^{3}\right)$ \\
\hline & 1 Hour & $35 \mathrm{ppm}\left(40 \mathrm{mg} / \mathrm{m}^{3}\right)$ \\
\hline \multirow{2}{*}{ Nitrogen Dioxide } & Annual Average & $0.053 \mathrm{ppm}\left(100 \mu \mathrm{g} / \mathrm{m}^{3}\right)$ \\
\hline & 1 Hour & \\
\hline \multirow{3}{*}{ Sulfur Dioxide } & Annual Average & $80 \mu \mathrm{g} / \mathrm{m}^{3}$ (0.03 ppm) \\
\hline & 24 Hour & $365 \mu \mathrm{g} / \mathrm{m}^{3}$ (0.14 ppm) \\
\hline & 1 Hour & \\
\hline \multirow{3}{*}{ Particulate Matter (PM10) } & Annual Arithmetic Mean & $50 \mu \mathrm{g} / \mathrm{m}^{3}$ \\
\hline & Annual Geometric Mean & \\
\hline & 24 Hour & $150 \mu \mathrm{g} / \mathrm{m}^{3}$ \\
\hline \multirow{2}{*}{ Particulate Matter - Fine (PM2.5) } & Annual Arithmetic Mean & $15 \mu \mathrm{g} / \mathrm{m}^{3}$ \\
\hline & 24 Hour & $65 \mu \mathrm{g} / \mathrm{m}^{3}$ \\
\hline Sulfates & 24 Hour & \\
\hline \multirow{2}{*}{ Lead } & Calendar Quarter & $1.5 \mu \mathrm{g} / \mathrm{m}^{3}$ \\
\hline & 30 Day Average & \\
\hline Hydrogen Sulfide & 1 Hour & \\
\hline Vinyl Chloride (chloroethene) & 24 Hour & \\
\hline Visibility Reducing particles & $\begin{array}{c}8 \text { Hour } \\
\text { (1000 to } 1800 \text { PST) }\end{array}$ & \\
\hline
\end{tabular}


Table V-B: California Air Quality Standards (SJVUAPCD)

\begin{tabular}{|c|c|c|}
\hline Pollutant & Averaging Time & Concentration \\
\hline \multirow{2}{*}{ Ozone } & 8 Hour & \\
\hline & 1 Hour & $0.09 \mathrm{ppm}\left(180 \mu \mathrm{g} / \mathrm{m}^{3}\right)$ \\
\hline \multirow{2}{*}{ Carbon Monoxide } & 8 Hour & 9.0 ppm $(10$ mg/m³) \\
\hline & 1 Hour & 20 ppm (23 mg/m³) \\
\hline \multirow{2}{*}{ Nitrogen Dioxide } & Annual Average & \\
\hline & 1 Hour & $0.25 \mathrm{ppm}\left(470 \mu \mathrm{g} / \mathrm{m}^{3}\right)$ \\
\hline \multirow{3}{*}{ Sulfur Dioxide } & Annual Average & \\
\hline & 24 Hour & $0.04 \mathrm{ppm}\left(105 \mu \mathrm{g} / \mathrm{m}^{3}\right)$ \\
\hline & 1 Hour & $0.25 \mathrm{ppm}\left(655 \mu \mathrm{g} / \mathrm{m}^{3}\right)$ \\
\hline \multirow{3}{*}{ Particulate Matter (PM10) } & Annual Arithmetic Mean & \\
\hline & Annual Geometric Mean & $30 \mu \mathrm{g} / \mathrm{m}^{3}$ \\
\hline & 24 Hour & $50 \mu \mathrm{g} / \mathrm{m}^{3}$ \\
\hline \multirow{2}{*}{ Particulate Matter - Fine (PM2.5) } & Annual Arithmetic Mean & \\
\hline & 24 Hour & \\
\hline Sulfates & 24 Hour & $25 \mu \mathrm{g} / \mathrm{m}^{3}$ \\
\hline \multirow{2}{*}{ Lead } & Calendar Quarter & \\
\hline & 30 Day Average & $1.5 \mu \mathrm{g} / \mathrm{m}^{3}$ \\
\hline Hydrogen Sulfide & 1 Hour & $0.03 \mathrm{ppm}\left(42 \mu \mathrm{g} / \mathrm{m}^{3}\right)$ \\
\hline Vinyl Chloride (chloroethene) & 24 Hour & $0.010 \mathrm{ppm}\left(26 \mu \mathrm{g} / \mathrm{m}^{3}\right)$ \\
\hline Visibility Reducing Particles & $\begin{array}{c}8 \text { Hour } \\
\text { (1000 to } 1800 \text { PST) }\end{array}$ & (See note 1 ) \\
\hline
\end{tabular}

ppm=parts per million

$\mathrm{mg} / \mathrm{m} 3=$ milligrams per cubic meter $\mu \mathrm{g} / \mathrm{m}^{3}=$ micrograms per cubic meter

Note 1: Statewide VRP Standard (except Lake Tahoe Air Basin): Particles in sufficient amount to produce an extinction coefficient of 0.23 per kilometer when the relative humidity is less than 70 percent. This standard is intended to limit the frequency and severity of visibility impairment due to regional haze and is equivalent to a 10-mile nominal visual range. 


\section{Air Quality Management Agencies}

1. Environmental Protection Agency and Other Federal Agencies

The EPA has primacy over air quality in the United States. It is the designated "Administrator" of the Federal Clean Air Act, which involves policy and research at the national level, as well as oversight of state and regional planning efforts. National level tasks are undertaken by EPA's headquarters (EPA HQ) in Washington, D.C., or at its research center in Research Triangle Park, N.C. Within the EPA Office of Air and Radiation (OAR) are programs for the criteria air pollutants, toxic air pollutants, visibility, indoor air quality, transportation, climate change, radiation, stratospheric ozone depletion, and acid deposition.

EPA provides new planning tools, such as models, as well as technical, policy and legal guidance. Especially relevant is the work undertaken by the Office of Air Quality Planning and Standards, a division OAR. It produces guidance for using models in air quality planning (for examples, see EPA, 1996b; 1999b, 1999c) and approves models for use.

Direct oversight of states' air quality planning practice is provided by EPA's regional offices. EPA’s Region IX office, located in San Francisco, oversees air quality planning in California, Arizona, Nevada, and Hawaii. It also has representatives on the Policy and Technical Committees overseeing air quality studies in Central California, which are described in the following chapter. 


\section{California Air Resources Board and Other State Agencies}

CARB is the state agency with dominion over California air quality. CARB

maintains primacy by being a step ahead of the EPA, thanks to California's long history

of air quality control, more stringent laws and rules, and considerable technical expertise.

Alas, another reason is the state's longstanding air quality problems. In

acknowledgement of CARB's successes, the FCAA specifically grants jurisdiction to

CARB.

In addition to the federal laws, CARB is responsible for enforcing the California

Clean Air Act. In some instances, CARB provides technical assistance to air district

creating and implementing plans. In all cases, CARB has authority to approve air

district's plans and, once approved, to forward them to EPA for final approval.

The 1994 SJV Ozone Attainment Demonstration Plan (94 Ozone Plan) provides a

succinct and clear description of the roles and responsibilities of federal and state

agencies in producing air quality attainment plans:

The EPA is the lead federal agency and is responsible for setting the NAAQS and establishing federal motor vehicle emission standards. The EPA is also responsible for reducing emissions from a number of sources including locomotives, aircraft, heavy duty vehicles used in interstate commerce, and other such sources which are either preempted from state control or best regulated on a national level. The EPA also has the authority under the FCAAA [Federal Clean Air Act Amendments] to require preparation of state plans for air quality and may approve or disapprove state air quality plans. The California Air Resources Board (ARB) is the lead state agency for air quality. The CARB is responsible for preparing and submitting a state air quality plan to the EPA. In preparing a state plan, the CARB reviews and approves regional air quality plans and incorporates them into a State Implementation Plan (SIP). Under state authority, CARB establishes emission standards for on-road motor vehicles and for consumer products sold in California. The air pollution control districts (APCDs) and air quality management districts (AQMDs) are responsible for developing that portion of the SIP that deals with stationary and area source controls 
and in cooperation with the transportation planning agencies (TPAs), the development of TCMs [Transportation Control Measures].

The FCAAA specifies that the Attainment Demonstration Plan must be submitted as revisions to the applicable SIP. The CARB is the mandated state agency for submission of SIP revisions. Therefore, after this Plan is adopted at the District level it will be submitted to the CARB for transmittal to the EPA. (SJVUAPCD, 1994)

Other state agencies have responsibilities pertaining to air quality planning in

California. The Bureau of Automotive Repairs oversees the motor vehicle inspection and maintenance programs. The California Highway Patrol, Department of Motor Vehicles (DMV) and Department of Transportation (CalTrans) are also involved in air quality control. For example, CalTrans provides vehicle activity data, such as miles traveled, and the DMV provides data about the number of drivers and types of vehicles in use.

\section{Air Districts}

California is divided into thirteen air districts, as shown in Figure V-1. These districts correspond roughly to air basins. The San Joaquin Valley Air Quality Study, described in more detail in the Air Quality Setting chapter, listed amongst its objectives understanding the transport of pollutants between air districts, notably between the Bay Area and the Central Valley (SJVAQS, 1996). Consequently, modeling studies often simulate more than one air basin. 
Figure V-1: California Air Districts (CARB, )

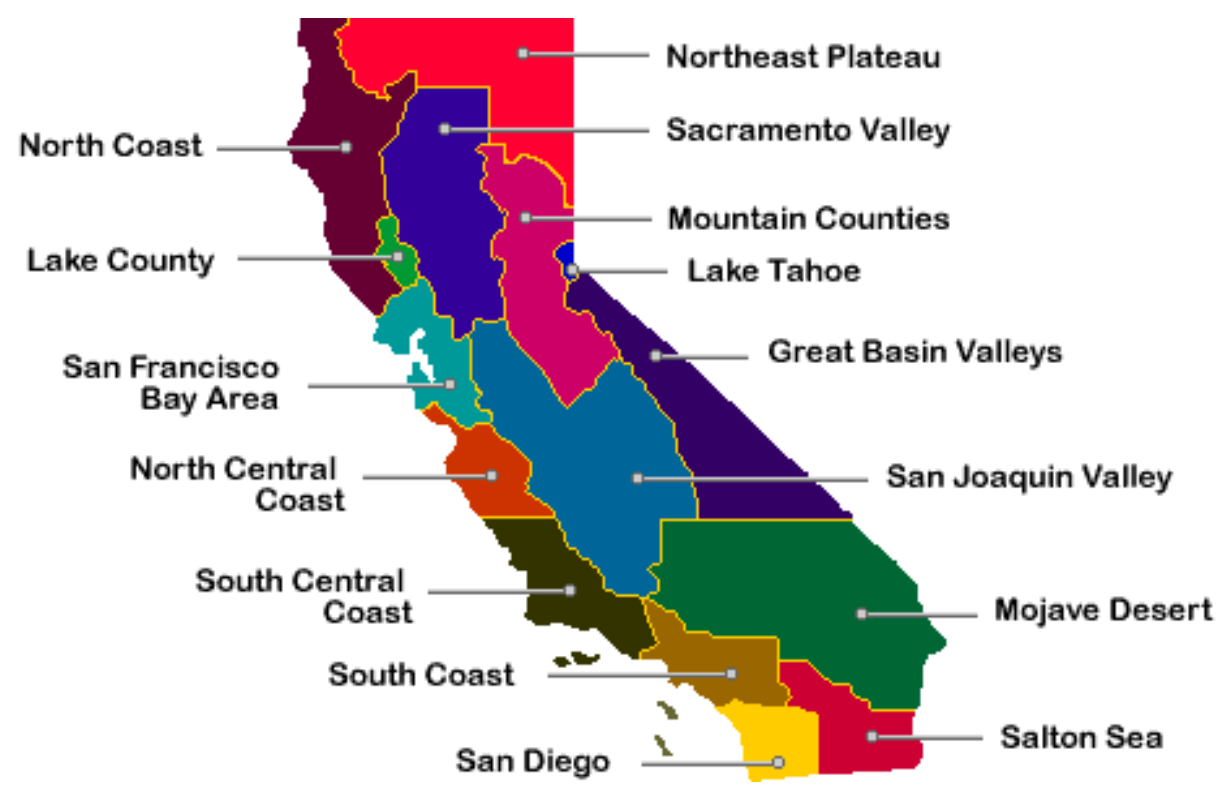

Air Districts are charged with managing air quality in specific air basins. Some air basins have one unified air district. The Bay Area Air Quality Management District covers the nine-country San Francisco Bay Area Air Basin. The San Joaquin Valley Unified Air Pollution Control District spans the eight counties of the SJV. See Figures V-2 and V-3 for maps of the BAAQMD and the SJVUAPCD, respectively.

The Air Districts of the Sacramento Valley Air Basin have not been unified. Instead, there are ten air districts, one for each county in the basin. ${ }^{134}$ The most active is the Sacramento Metropolitan Air Quality Management District (SMAQMD). It participates in ongoing Central Valley air quality studies, since it has ongoing air quality concerns.

Though it had a limited role in Central California air quality planning during the 1990's, the SMAQMD is related to the BAAQMD and SJVUAPCD. It commented on

\footnotetext{
${ }^{134}$ Although part of Solano Country is in the Sacramento Valley Air Basin, it is within the jurisdiction of the Bay Area Air Quality Management District. Sutter and Butte Counties do not have air quality
} 
Bay Area and SJV air quality plans because it is occasionally downwind (or upwind) of the SJV or the Bay Area. Like the SJVUAPCD, the SMAQMD has a pending lawsuit to force the BAAQMD to implement the enhanced vehicle checking program (SMAQMD 2002).

Figure V-2: Bay Area Air Quality Management District (BAAQMD)

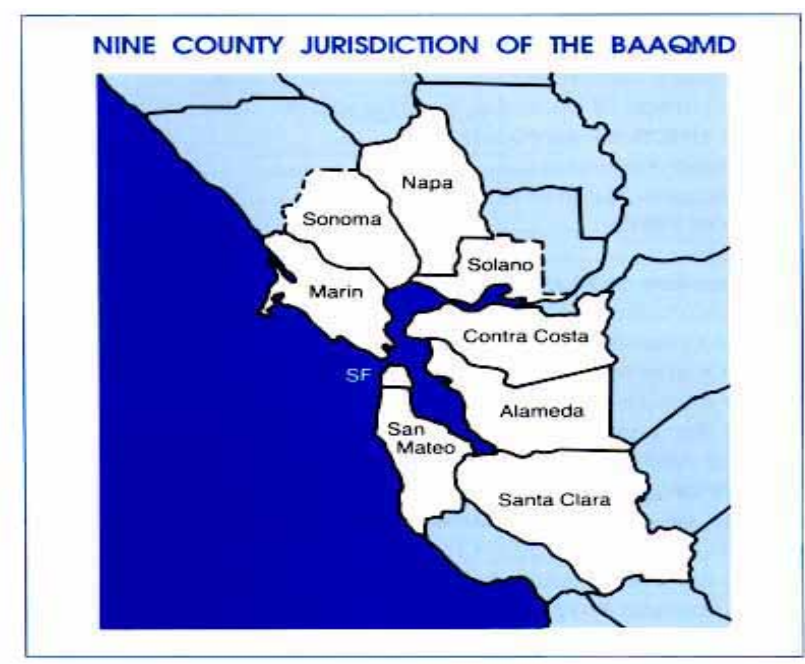


Figure V-3: SJV Unified Air Pollution Control District (SJVUAPCD, )

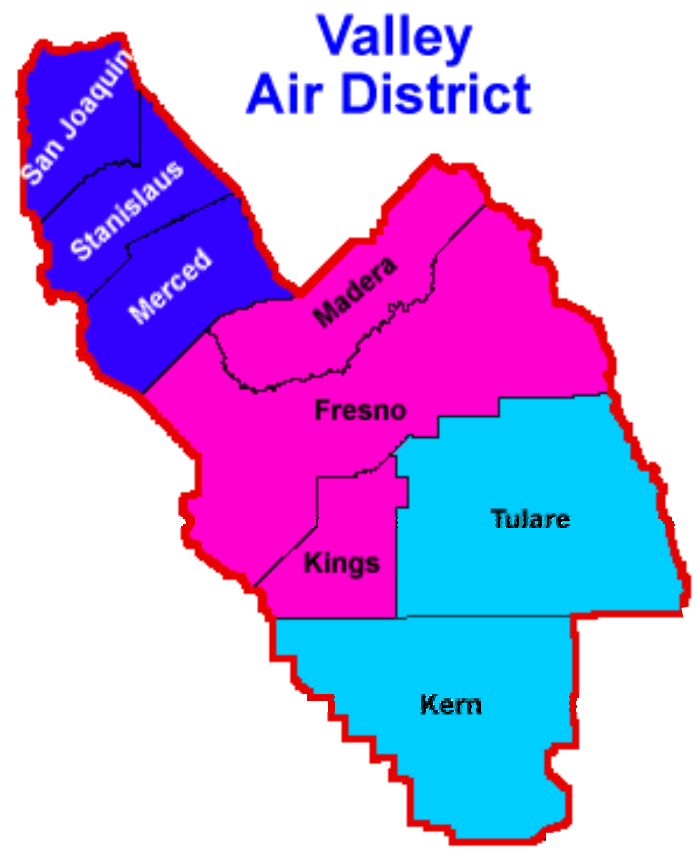

Per the CCAA requirements for assessing transport relationships, CARB concluded in the early 1990's that the Bay Area and SJV air basins contribute pollution to each other. The direction of flow of particular concern is prevailing westerly wind from the Bay Area into the Central Valley. Of this relationship, the SJVUAPCD's web site says in answer to the question, "How much [air pollution] comes from other areas?” (SJVUAPCD),

Air pollution transported from the San Francisco Bay and Sacramento areas account for approximately $27 \%$ of the total emissions in the Northern portion of the District (San Joaquin, Stanislaus, and Merced Counties). In the Central region (Fresno, Madera and Kings Counties), the percentage drops to $11 \%$, and in the south valley (the Valley portion of Kern and Tulare Counties), transported air pollution accounts for only $7 \%$ of the total problem. While some of our pollution is blown in from other areas, most of our air pollution is home grown and it is our responsibility to clean it up.

The scientific basis of the SJV's statement is modeling conducted by CARB.

Discussed in considerable detail in the chapters that follow, the numbers presented by the SJVUAPCD are uncertain. The importance of the relationship, and agency decisions 
based on it, are examined in detail in this research. Questionable quantification

notwithstanding, there is general agreement that some relationship between the two air basins exists.

\section{Local Planning Agencies}

There are, of course, local planning agencies in addition to air districts. There are six metropolitan planning organizations (MPOs) and two rural transportation planning agencies in the SJV. These agencies are referred to as Councils of Governments (COGs) because they include city and county governments, and sometimes transit and ridesharing agencies and public interest groups. In the Bay Area, these councils are unified into a land use agency (Association of Bay Area Governments, ABAG) and a transportation agency (Metropolitan Transportation Council, MTC).

These organizations work, both formally and informally, with air districts on clean air planning. The COGs help to develop Reasonably Available Control Measures (RACM) for reducing emissions from transportation. Transportation control measures differ from technology and fuel-based emissions controls on motor vehicles.

Another responsibility of transportation planning agencies is transportation plans with measures that "conform" with clean air plans. Failure to do so causes a "conformity lapse” and attendant freezing of federal funds for transportation improvements. Consequently, the need for conformity provides impetus to complete both ozone and transportation plans within deadlines. 
COGs also provide input data for modeling studies. For example, they provide data about current and projected land use, population, and pollution-causing activities, such as driving and industrial development.

\section{Planning Requirements}

\section{Ozone Attainment Demonstration Plans}

Violating the NAAQS triggers a requirement for air districts to submit a plan for cleaning up the air, mostly via emissions reductions. Plans are aggregated and codified as a State Implementation Plan. The pollutant-specific plans for each air district violating a NAAQS are grouped and submitted as the SIP. For example, the San Joaquin Valley's 94 Ozone Plan was submitted with plans from other air districts to comprise the 1994 California Ozone SIP.

The FCAA specifies planning requirements, including deadlines for plan submission and goal attainment, depending on the severity of the air quality problem (see Table V-D, below). When the SJVUAPCD developed its 94 Ozone Plan, it was classified as a “serious” nonattainment area for the ozone NAAQS. The classification required the SJVUAPCD to meet the standard by November 15, 1999. Unfortunately and despite the 94 Ozone Plan, air quality did not meet the standard by 1999. Consequently, on October 23, 2001, the EPA officially reclassified the SJV air basin as "severe” nonattainment (SJVUAPCD 2001; EPA 2001).

Areas designated severe nonattainment for ozone are given until 2005 to meet the $\mathrm{O}_{3}$ NAAQS. The SJVUAPCD is now considering another redesignation; this time to an “extreme” nonattainment classification. This voluntary action is being contemplated 
because the 2005 attainment is deadline viewed as “impossible” (SJVUAPCD 2001c). An extreme area has until 2010 to meet the standard. Furthermore, extreme areas may promulgate an attainment plan that calls for emissions reductions without specifying the sources and means of achieving those reductions. Further implications for nonattainment classifications are summarized in Table V-D.

The Bay Area was designated as attainment for the ozone NAAQS in 1995, but experienced enough violations of the standard subsequently to be reclassified as nonattainment by EPA in 1998 (EPA 1999a). Given the Bay Area's flirtation with the ozone NAAQS, EPA did not classify the airshed beyond nonattainment (e.g., serious, severe, extreme). 


\section{Table V-D: Requirements Per Ozone NAAQS Nonattainment Classification}

(SJVUAPCD 2001)

\begin{tabular}{|c|c|c|}
\hline $\begin{array}{l}\text { Nonattainment } \\
\text { Classification }\end{array}$ & Requirements & Description \\
\hline \multicolumn{3}{|l|}{ Serious } \\
\hline & $\begin{array}{l}\text { Major Stationary Source } \\
\text { Definition }\end{array}$ & $\begin{array}{l}\text { Any source in the air basin that emits at least } 50 \\
\text { tons per year of } \mathrm{NO}_{\mathrm{x}} \text { or VOCs is subject to permit } \\
\text { and control requirements. }\end{array}$ \\
\hline & Enhanced Monitoring & $\begin{array}{l}\text { The attainment plan must include measures to } \\
\text { improve monitoring of ambient ozone and its } \\
\text { precursors. }\end{array}$ \\
\hline & $\begin{array}{l}\text { Attainment and Reasonable } \\
\text { Further Progress } \\
\text { Demonstrations }\end{array}$ & $\begin{array}{l}\text { Air districts must submit plans that demonstrate } \\
\text { attainment of the standard by November 15, } 1999 \\
\text { and reasonable further progress annually, defined } \\
\text { as reducing baseline VOC emissions by three } \\
\text { percent per year. }\end{array}$ \\
\hline & $\mathrm{NO}_{\mathrm{x}}$ Control & $\begin{array}{l}\text { Reasonable further progress may be demonstrated } \\
\text { with } \mathrm{NO}_{\mathrm{x}} \text { emissions reductions in lieu of VOC } \\
\text { reductions, as long as the result in ozone } \\
\text { concentrations is at least equivalent to VOC } \\
\text { reductions. }\end{array}$ \\
\hline & Offset Requirement & $\begin{array}{l}\text { New or modified sources must offset any new } \mathrm{NO}_{\mathrm{x}} \\
\text { or VOC emissions at a ratio of } 1.2 \text { to } 1 .\end{array}$ \\
\hline \multicolumn{3}{|l|}{ Severe } \\
\hline & Attainment Deadline & November 15, 2005 \\
\hline & Serious Area Requirements & $\begin{array}{l}\text { All requirements for serious nonattainment areas } \\
\text { apply, except some become more stringent. }\end{array}$ \\
\hline & $\begin{array}{l}\text { Major Stationary Source } \\
\text { Definition }\end{array}$ & $\begin{array}{l}\text { Extends to include sources emitting at least } 25 \text { tons } \\
\text { per year of } \mathrm{NO}_{\mathrm{x}} \text { or VOC. }\end{array}$ \\
\hline & Offset Requirement & $\begin{array}{l}\text { New or modified sources must offset any new } \\
\text { emissions at a ratio of } 1.3 \text { to } 1 \text {. If all major } \\
\text { sources in the area use Best Available Control } \\
\text { Technology, then the offset ratio remains } 1.2 \text { to } 1 .\end{array}$ \\
\hline & Major Modification Deadline & $\begin{array}{l}\text { A major modification to an existing operation is } \\
\text { defined as increasing emissions by } 25 \text { tons over } 5 \\
\text { years. }\end{array}$ \\
\hline \multicolumn{3}{|l|}{ Extreme } \\
\hline & Attainment Deadline & November 15, 2010 \\
\hline & $\begin{array}{l}\text { Major Stationary Source } \\
\text { Definition }\end{array}$ & $\begin{array}{l}\text { Extends to include sources emitting at least } 10 \text { tons } \\
\text { per year of } \mathrm{NO}_{\mathrm{x}} \text { or VOC. }\end{array}$ \\
\hline & Black Box & $\begin{array}{l}\text { Plan may call for emissions reductions without } \\
\text { specifying how those reductions will be achieved. }\end{array}$ \\
\hline & Offset Requirement & $\begin{array}{l}\text { New or modified sources must offset any new } \\
\text { emissions at a ratio of } 1.5 \text { to } 1 \text {. }\end{array}$ \\
\hline & Major Modification Deadline & $\begin{array}{l}\text { Any modification is considered a major } \\
\text { modification (i.e., there is no de minimis level). }\end{array}$ \\
\hline
\end{tabular}




\section{Modeling Requirements for Ozone Attainment Demonstration Plans}

Both the complexity of ozone formation and regulatory requirements compel air basins to study ozone using photochemical air quality simulation models (PAQSM). Laws and regulations have grown to rely upon and require the use of PAQSM for planning purposes. The 1990 Amendments to the FCAA require the use of PAQSM in areas designated as serious, severe or extreme nonattainment. ${ }^{135}$ The legislation includes language such as:

Areas exceeding the ozone NAAQS shall provide...."a demonstration that the (State Implementation) plan will provide for attainment of the ozone NAAQS. This demonstration must be based on photochemical grid modeling or any other analytical method determined by the Administrator...to be at least as effective” (italics added, §182 of CAAA 1990).

The technical bases of an ozone plan, particularly the modeling simulation to show how future air quality will meet the NAAQS, is a key intersection of science and planning. The 94 Ozone Plan describes how modeling was used for plan development (SJVUAPCD 1994, page ii):

Serious nonattainment areas must demonstrate by computer modeling that the federal ozone standard will be obtained by 1999. This component must include all possible control measures necessary to make attainment. The Attainment Demonstration Plan uses a computer model to simulate the future air quality in the Valley while reflecting the effects of measures proposed to curb pollution. The model is complex, new, state of the art, and undergoing continuous refinement. Nevertheless, it is expected that the model will serve as the preeminent tool for local, state and federal agencies, the public, and industry, to evaluating current and proposed air quality planning efforts. The model has predicted District-wide attainment of the federal ozone standard by 1999 .

Of course, the above text describes a process that complies with the FCAA. The way the models are really used is a key question of this research. The specificity of the 1990

\footnotetext{
${ }^{135}$ Serious, severe and extreme nonattainment areas have peak ozone that exceeds the $120 \mathrm{ppb}$ NAAQS by
} 
CAA and associated guidance by EPA and CARB about the proper use of modeling create modeling practice and apparent model-based planning that belie the limitations of the PAQSM.

\section{Modeling Requirements for Clean Air Plans}

The ozone management plan required by the CCAA, Clean Air Plan (CAP), does not include a modeling demonstration like required for federal plan. Models need not be used for CAP development.

Modeling simulations may be used to show that the state ozone standard will be met in a reasonable timeframe, but no district can identify adequate emissions reductions to simulate. None have identified enough emissions controls that, when simulated through modeling, will indicate that the $90 \mathrm{ppb}$ ozone standard will be attained. More precisely, no air district has identified enough controls that they are willing to attempt to implement.

\section{Modeling Requirements for Transport Assessment}

Where an upwind air basin is implicated for poor air quality downwind, it must "establish mitigation requirements commensurate with the level of contribution" (CCAA 2001). In California during the mid-1990's that mitigation took the form of reducing both hydrocarbon and $\mathrm{NO}_{\mathrm{x}}$ emission from stationary sources by 75 percent unless it could be demonstrated that some other reductions were equal (Ziman 2000). Modeling was the tool used for that demonstration.

Thus, modeling may serve two purposes in transport assessment and mitigation. First, it can be used to evaluate the impact of upwind emissions on downwind air quality. 
Second, it can be used to evaluate the efficacy of upwind emissions controls. Neither of these uses, however, is explicitly required by the language of the CCAA.

\section{E. Summary}

This chapter provides an overview of the legal and planning requirements for clean air in Central California. Responsible agencies and their responsibilities are identified, including requirements using models. The main points of this chapter include:

- The FCAA and CCAA each create the impetus for air quality planning in Central California, but their approaches are different. The FCAA is quite specific and includes penalties for noncompliance, whereas the CCAA sets no deadlines and threatens no sanctions.

- Federal and state laws set forth ambient air quality standards, as well as methods for planning to meet these standards. Federal planning requirements depend, in part, on the severity of the air quality problem.

- The CCAA ozone air quality standard is more stringent than the federal standard, but requirements for planning to meet the standard are less prescriptive. Whereas the FCAA specifies attainment deadlines depending on the severity of the ozone problem, no deadlines are set forth in the CCAA. Also, the FCAA specifies penalties for failure to promulgate plans or to meet standards within specified timelines. The CCAA involves no such deadlines or penalties.

- Federal, state and local agencies have roles pertaining to air quality in Central California, creating a web of responsibilities and interactions. EPA engages planning through regional offices primarily, which is Region IX for Central California. The division of responsibilities for planning gives CARB dominion over California, but gives local air districts the responsibility for producing air quality improvement plans.

- New standards promulgated by the U.S. EPA for peak ozone averaged over 8hours and particulate matter smaller than 2.5 microns in diameter will represent new planning challenges, as both standards are currently violated in the Bay Area and the SJV.

- The Bay Area and SJV air basins are interdependent "transport couples” due to the significant transfer of pollutants. Generally, the Bay Area is considered the upwind culprit. 
- Due in part to the requirements of the FCAA, the use of models has grown over the past few decades.

- The FCAA calls explicitly for the use of photochemical air quality simulation modeling to "demonstrate" that air quality plans will yield acceptably clean air by a specified date. The CCAA does not require modeling; rather, it encourages state standards to be met as fast as reasonably practicable. Modeling has a more important role in transport assessment and mitigation, per the CCAA, whereby it may be used to evaluate transport couples, as well as the efficacy of possible mitigation measures. 


\section{APPENDIX C: CENTRAL CA SOCIOECONOMIC SETTING}

\section{A. Introduction}

This chapter describes the geography, demographics and socioeconomic conditions in Central California. The primary region of focus is the San Joaquin Valley. The San Francisco Bay Area is of secondary interest, mostly as it relates and compares to the SJV. The chapter ends with a discussion of planning challenges in the SJV that puts air quality concerns in perspective relative to socioeconomic and other natural resource planning issues.

\section{B. Geographic Setting}

"Central California” is the center portion of California. It is a rectangular area that stretches northwest to Mendocino, southwest to San Luis Obispo, northeast beyond Lake Tahoe, and southeast to Death Valley National Monument. It includes the San Francisco Bay, portions of the coastal and Sierra mountain ranges, and the valley formed in the trough of the mountain ranges. The Great (or Central) ${ }^{136}$ Valley includes both the Sacramento and San Joaquin Valleys and is 450 miles long but only 40 to 60 miles wide.

Although only a part of Central California, the SJV is the primary focus of this case study. It includes eight counties covering approximately 27,000 square miles, which is an area bigger than New Jersey. It is the nation’s most productive agricultural region, producing \$6.6 billion in agricultural products in 1997 (USDOA 1997). Its unique combination of topography, meteorology and air pollutant emissions create some of the nation’s worst ambient air quality conditions.

\footnotetext{
136 The term "Central Valley” is often used to refer to the entire Great Valley or only the San Joaquin Valley. Throughout this text, Central Valley will be used to refer to the Great Valley; that is, the region
} 
As shown in Figure APPC-1, the San Joaquin Valley is bordered on three sides by mountains; the Sierra Nevada range to the East, the Diablo and Temblor Ranges to the West, and the Tehachapi Mountains to the South. The northern boundary of San Joaquin Valley is the Sacramento Valley. The figure reveals how the Central Valley is a large basin capable of capturing and holding pollutant emissions, thereby allowing them to heat up and react chemically. In addition, the SJV is very hot and sunny. In Fresno, the average maximum daily temperatures in July is $98.6^{\circ} \mathrm{F}$ (Abstract 2001). The SJV averages 40 days per year hotter than $100^{\circ} \mathrm{F}$ (SJVUAPCD 2001). These conditions are excellent for making ozone, an air pollutant occurring in concentrations above the Federal and State standards on 30 and 114 days, respectively, in the SJV in 2000.

The State of the Great Central Valley of California summed up the situation,

The geography that defines the Valley and which contributes to many of its positive attributes also creates a collection point for air pollutants that originate from both from within the Valley and from the San Francisco Bay Area (GVC).

As neighbors to the SJV, the Bay Area (and Sacramento) are of interest as upwind contributors of air pollution to the SJV. 


\section{Figure APPC-1: California's Central Valley (Sterner)}

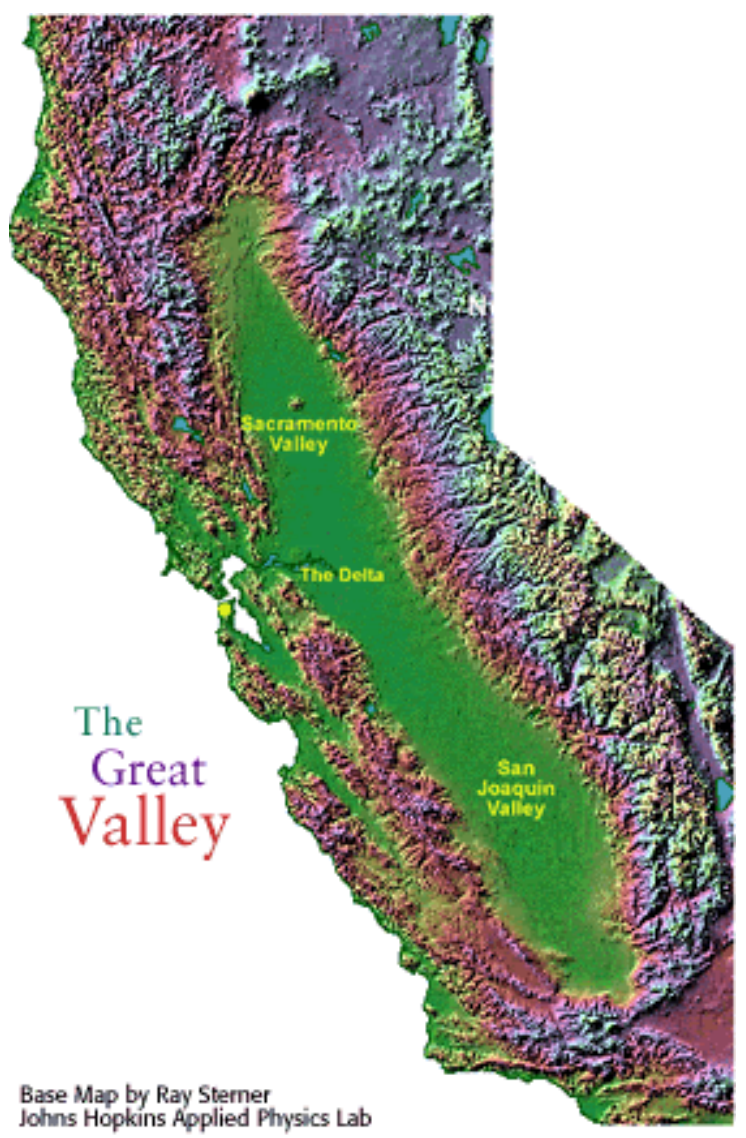

\section{Modeling Domains}

For the purposes of this case, Central California is defined by the area simulated (i.e., domain) by the SARMAP modeling system, which was developed in the early 1990's as part of the San Joaquin Valley Air Quality Study. The SARMAP domain extends beyond the mountain ranges approximately 50 miles into the Pacific Ocean, and into Nevada, as shown in Figure APPC-2. The northern boundary is approximately 100 miles north of Sacramento. Edwards Air Force Base and China Lake demarcate the southern boundary.

The ongoing Central California Ozone Study (CCOS) is intended to extend the findings of the SJVAQS, including new modeling. This time, the modeling will 
incorporate a larger domain than the original SARMAP application, including a western boundary approximately 200 miles into the Pacific Ocean and a northern boundary well north of Sacramento. Thus, the area included in the modeling analysis of this region is expanding. Despite a changing modeling domain, the sociopolitical and demographics characteristics of the case remain consistent. Expanding the domain used for CCOS adds sparsely populated areas north of Sacramento, and ocean west of the California coast.

The impetus for using a larger modeling domain in CCOS is uncertainty about the air quality conditions at the domain borders. Extending further west and north makes it more defensible to assume "clean" boundary conditions, which ambient chemical species concentrations associated with the free troposphere. That is, air masses blowing into the domain from the west can be assumed to have traveled thousands of miles across the Pacific Ocean and, as such, are not influenced by pollution blown off the California coast from coastal urban areas. 
Figure APPC-2: SARMAP Modeling Domain

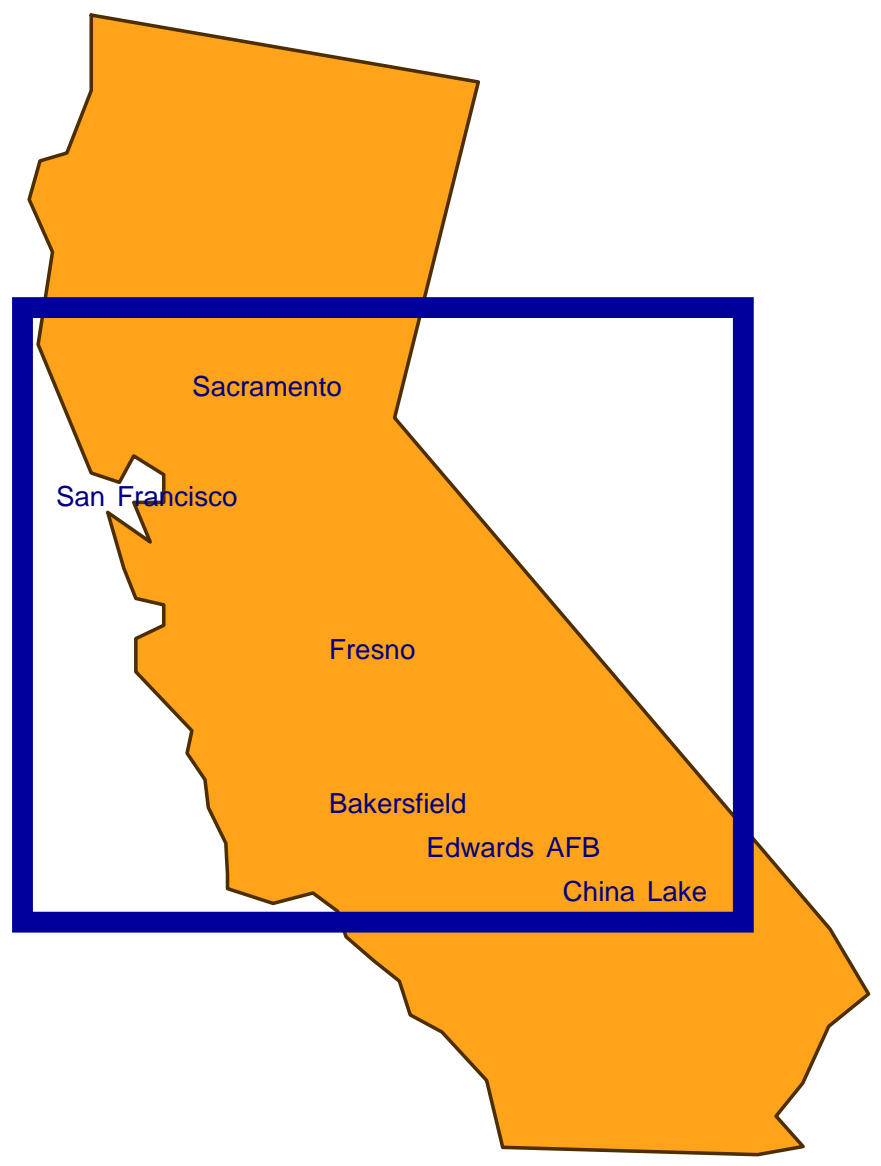




\section{Demographic and Socioeconomic Setting}

\section{Population}

Population and air quality are related. Where there are lots of people, associated emissions degrade the quality of the air. Not surprisingly, most counties in the U.S. that violate ozone and PM NAAQS contain cities. Central California is home to several major metropolitan areas. The largest cities are listed in Table APPC-A.

Table APPC-A: Major Cities in Central California

\begin{tabular}{|c|c|c|c|}
\hline Air District & City & County & $\begin{array}{l}2000 \text { Population } \\
\text { (Census 2000) }\end{array}$ \\
\hline \multicolumn{4}{|l|}{ BAAQMD } \\
\hline & Berkeley & Alameda & 103,000 \\
\hline & Concord & Contra Costa & 122,000 \\
\hline & Freemont & Alameda & 203,000 \\
\hline & Hayward & Alameda & 140,000 \\
\hline & Oakland & Alameda & 399,000 \\
\hline & Richmond & Contra Costa & 99,000 \\
\hline & San Francisco & San Francisco & 777,000 \\
\hline & San Jose & Santa Clara & 895,000 \\
\hline & Santa Clara & Santa Clara & 102,000 \\
\hline & Santa Rosa & Sonoma & 148,000 \\
\hline & Sunnyvale & Santa Clara & 132,000 \\
\hline & Vallejo & Solano & 117,000 \\
\hline \multicolumn{4}{|l|}{ SJVUAPCD } \\
\hline & Bakersfield & Kern & 247,000 \\
\hline & Fresno & Fresno & 428,000 \\
\hline & Modesto & Stanislaus & 189,000 \\
\hline & Stockton & San Joaquin & 244,000 \\
\hline & Visalia & Tulare & 92,000 \\
\hline SMAQMD & Sacramento & Sacramento & 407,000 \\
\hline
\end{tabular}

BAAQMD = Bay Area Air Quality Management District

SJVUAPCD = San Joaquin Valley Unified Air Pollution Control District

SMAQMD = Sacramento Metropolitan Air Quality Management District

Counties in Central California are shown in Table APPC-B, as are their populations

in 2000. The nine counties of the BAAQMD are home to twice as many residents as the eight counties within the SJVUAPCD. The people in the Bay Area also live closer 
together. In the BAAQMD, there are approximately 980 people per square mile compared with only 121 people per square mile in the SJVUAPCD. ${ }^{137}$

Table APPC-B: Central California Counties and Their Populations

\begin{tabular}{|l|l|r|}
\hline Air District & County & $\begin{array}{r}\text { 2000 Population } \\
\text { (Census 2000) }\end{array}$ \\
\hline BAAQMD & & $\mathbf{6 , 7 8 5 , 0 0 0}$ \\
\hline & Alameda & $1,444,000$ \\
\hline & Contra Costa & 949,000 \\
\hline & Marin & 247,000 \\
\hline & Napa & 124,000 \\
\hline & San Francisco & 777,000 \\
\hline & San Mateo & 707,000 \\
\hline & Santa Clara & 1683,000 \\
\hline & Solano & 395,000 \\
\hline & Sonoma & 459,000 \\
\hline & & $\mathbf{3 , 3 0 3 , 0 0 0}$ \\
\hline SJVUAPCD & Fresno & 799,000 \\
\hline & Kern & 662,000 \\
\hline & Kings & 129,000 \\
\hline & Madera & 123,000 \\
\hline & Merced & 211,000 \\
\hline & San Joaquin & 564,000 \\
\hline & Stanislaus & 447,000 \\
\hline & Tulare & 368,000 \\
\hline
\end{tabular}

BAAQMD = Bay Area Air Quality Management District

SJVUAPCD = San Joaquin Valley Unified Air Pollution Control District

SMAQMD = Sacramento Metropolitan Air Quality Management District

The Bay Area has a longer history of air quality concerns and, consequently, a longer record of air quality observations and planning efforts. The SJVUAPCD was formed in 1992 to unify county-level air quality management efforts. Comparatively, the BAAQMD became the state's first regional air quality management agency when the California Legislature formed it in 1955.

\footnotetext{
${ }^{137}$ Based on 2000 population (Census 2000) and 1999 county land areas (Abstract 2001). Water area in counties is not included in the calculation of area.
} 


\section{Growth}

All of Central California experienced dramatic growth in the 1900’s. As shown in Figure APPC-3, the BAAQMD experienced faster rates of growth in the earlier decades, but the SJVUAPCD grew faster since 1970. Over half of the population in the SJVUAPCD arrived since 1970, compared with only about 30 percent in the BAAQMD.

Figure APPC-3: Decennial Population Growth in Air Districts (Census 2000)

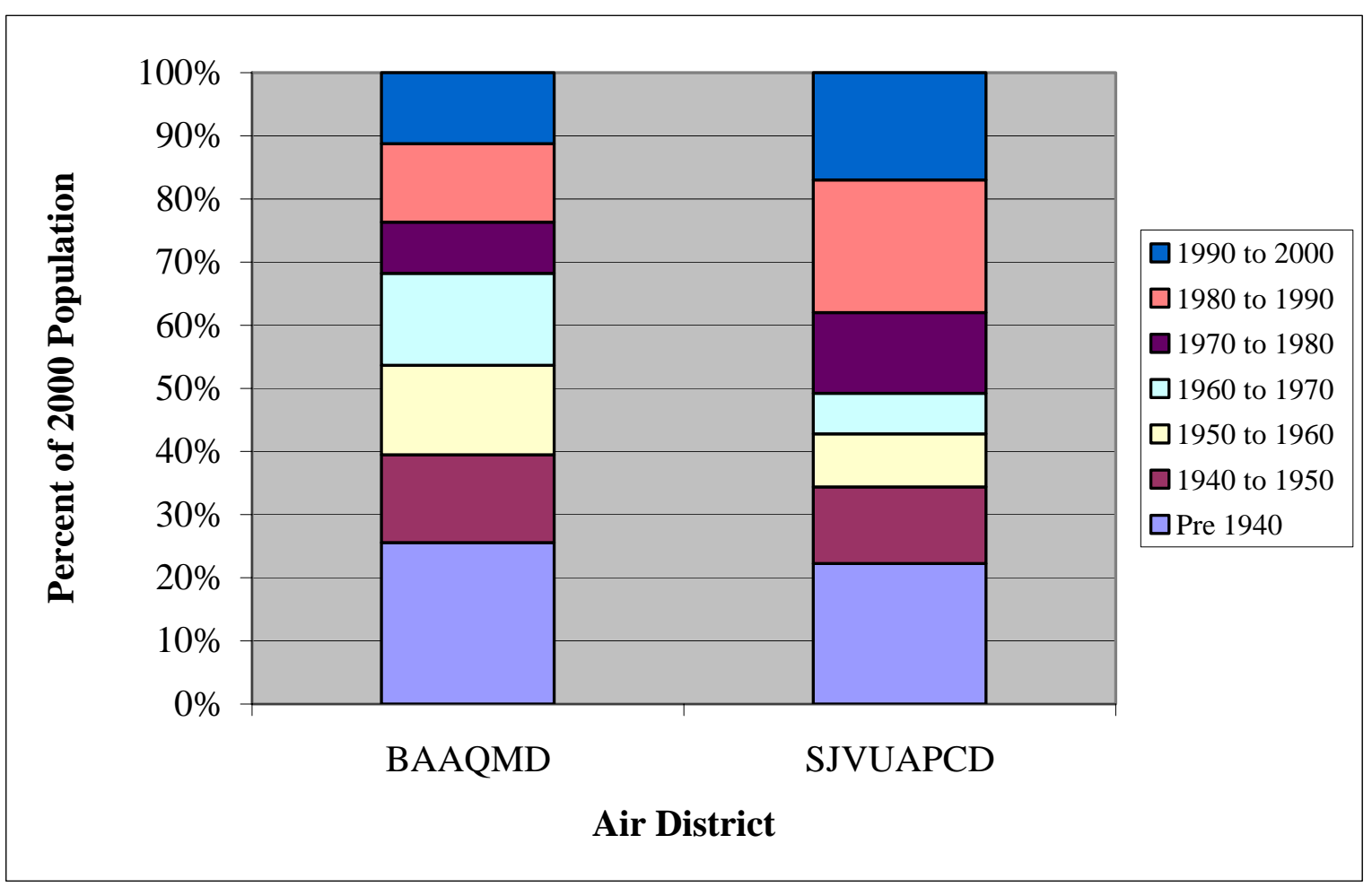

Projections indicate that the SJV will continue to grow at a dramatic rate. Estimates by the California Department of Finance, which build upon 1990 U.S. Census data, indicate that the SJV population will more than double from 3,375,000 in 2000 to 7,303,000 by 2040. How good are these projections? Data from the 2000 Census reveal that the population is $3,303,000$, not the projected $3,375,000$, a difference of a mere two percent. 
During the past few decades, air quality concerns in the SJVUAPCD have become a planning concern. This is no coincidence. People and their activities are responsible for pollutant emissions, which are discussed in more detail in the next chapter. As greater attention was focused on the SJV, more information was collected about the severity of air quality conditions. Exacerbating this concern is expected further population growth. Thus, as population grows, so too would emissions if not for reductions measures implemented as part of air quality management and planning.

\section{Composition}

The SJV has a high percentage of minorities, and the percentage is growing. As shown in Figure APPC-4, the SJVUAPCD has a higher percentage of people who consider themselves Hispanic or Latino than the BAAQMD or California overall.

Figure APPC-4: Population Considered Hispanic in 2000 (Census 2000)

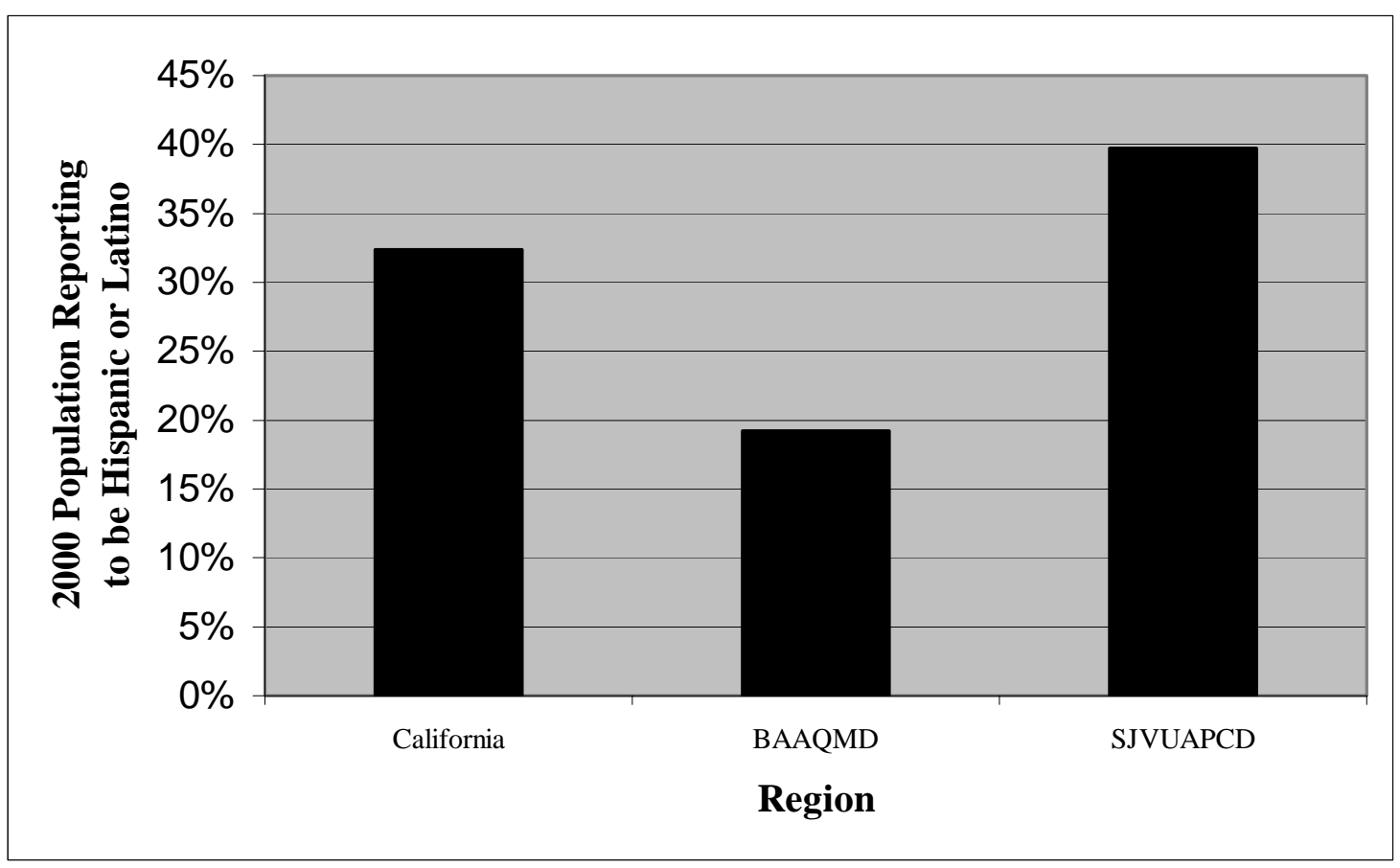


The U.S. Bureau of Census considers Hispanics to be of any race. This makes it difficult to estimate the percentage of minorities in the SJV, because Hispanics may be considered white in the 2000 Census. Confounding data notwithstanding, the minority percentages are shown in Figure APPC-5. The Bay Area has a higher percentage of people who consider themselves as black or African American, Asian, or Hawaiian or other Pacific Islander. The SJV has a much higher percentage of people who consider themselves neither one of these minority groups nor white. Presumably, many of these people are Hispanic or Latino. Furthermore, the SJV is believed to be home to many migrant workers and illegal aliens who are not counted by the formal U.S. Census (see, for example, Sherman 1997).

Minority populations are growing much faster than white populations in the SJV. Projections from the California Department of Finance indicate that nearly 70 percent of the population will be minority (i.e., Hispanic, Black, Asian, Pacific Islander, or American Indian) by 2040, as shown in Figure APPC-6. 
Figure APPC-5: Percent Minorities in 2000

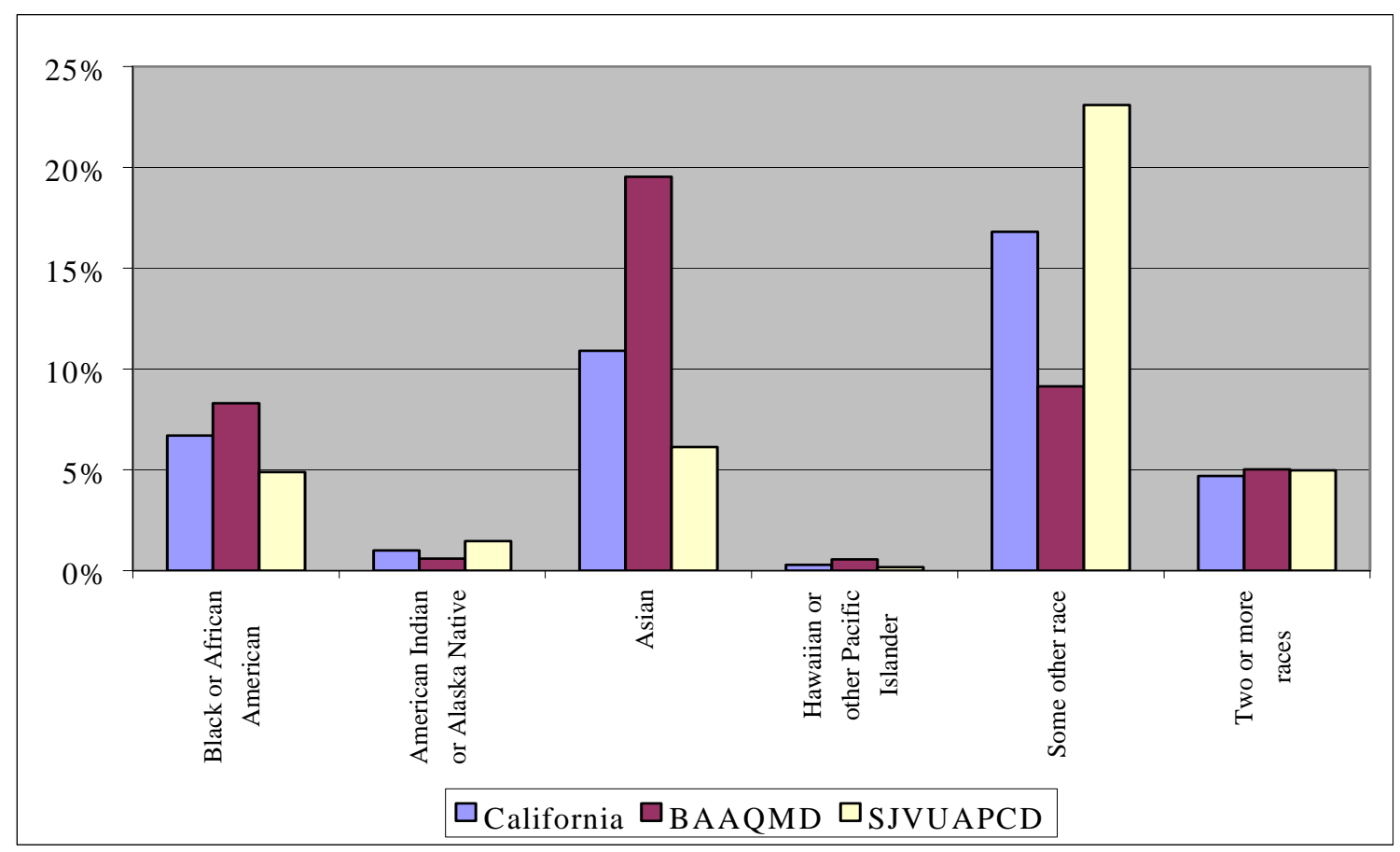

Figure APPC-6: Projected Composition of the SJV Population in 2000 and 2040 (Heim 1998)

\begin{tabular}{|c|c|c|}
\hline \multicolumn{3}{|l|}{$100 \%$} \\
\hline \multicolumn{3}{|l|}{$60 \%$} \\
\hline \multicolumn{3}{|l|}{$40 \%$} \\
\hline \multicolumn{3}{|l|}{$20 \%$} \\
\hline $0 \%$ & 2000 & 2040 \\
\hline 口American Indian & 28,000 & 56,000 \\
\hline$\square$ Black & 149,000 & 337,000 \\
\hline$\square$ Asian or Pacific Islander & 273,000 & 821,000 \\
\hline$\square$ Hispanic & $1,153,000$ & $3,714,000$ \\
\hline$\square$ White & $1,771,000$ & $2,379,000$ \\
\hline
\end{tabular}

Unfortunately, the 2000 Census data about race and ethnicity cannot be compared directly with the projections derived from the 1990 Census because they were gathered 
using different methods. For example, the 2000 Census includes categories, such as “Persons reporting two or more races”, whereas the California Department of Finance data, which were derived from the 1990 Census, do not. Nonetheless, the SJV's racial composition per the 2000 Census is shown in Figure APPC-7. These data also demonstrate the significance of the minority population in the region, although it is not as extreme as projected.

Figure APPC-7: Composition of SJV Population in 2000 (Census 2000)

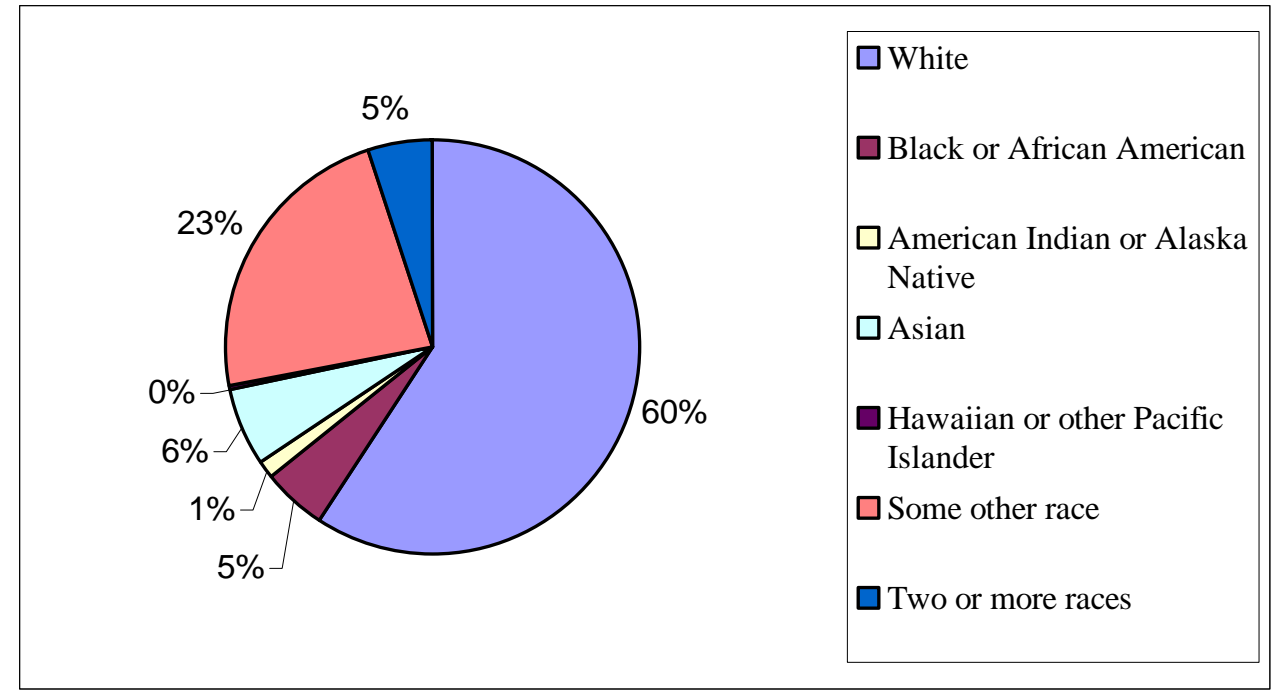

Information about the population indicates what planning concerns are likely to be priorities. Anticipated population growth is relevant because emissions will grow too, unless measures are taken to abate them. Similarly, description and comparison of the ethnic composition informs discussion of how the local politics may differ in the SJV and Bay Area, such as the potential for environmental injustices.

Mr. Kip Lipper, consultant to the state Senate Environmental Quality Committee and an author of the California Clean Air Act of 1988, said, 
The politics on air pollution has changed a great deal over the last decade. For the people who advocate air-quality issues in the Legislature, there is a distinct Latino and children's health focus and concern about environmental justice. The traditional approach to reducing air pollution is not as much in the forefront (Polakovic 2001).

The following description of the economic and health status of the SJV population further informs understanding of the relationship between population characteristics and the impetus and prospects for air quality management.

\section{Income, Education and Health}

In addition to being largely and increasingly minority, the SJV is dominated by lowincome communities poorer than average for California or the Bay Area. Relative to the Bay Area and California as a whole, the residents of the San Joaquin Valley are poorer, less employed and less educated. In the following discussion, aggregate figures are presented for the all nine counties in the SJV. All of the counties have the same characteristics, more or less, with respect to these socioeconomic measures. For a county-specific assessment, refer to Umbach (1997) or Penbera (1996).

Indicators suggest SJV residents have less access to health care than other Californians. They have fewer physicians and hospital beds per capita, less adequate prenatal care, and a higher rate of births to adolescent mothers (Umbach 1997).

As shown in Figure APPC-8, the median household income in the SJV was \$32,353 in 1997, which was 18 percent lower than the California median of $\$ 39,595$ and a whopping 37 percent below the Bay Area median of $\$ 51,687$. Figure APPC-9 reveals that more than twice as many people in the SJV live in poverty than in the Bay Area, where poverty is defined by the U.S. Census in terms of family income thresholds as a 
function of household size and composition. ${ }^{138}$ For example, a family consisting of a mother, father, two children and great-aunt had a poverty income threshold of \$21,665 in 2001.

Figure APPC-8: Median Household Income, 1997 Model-based (Census 2000)

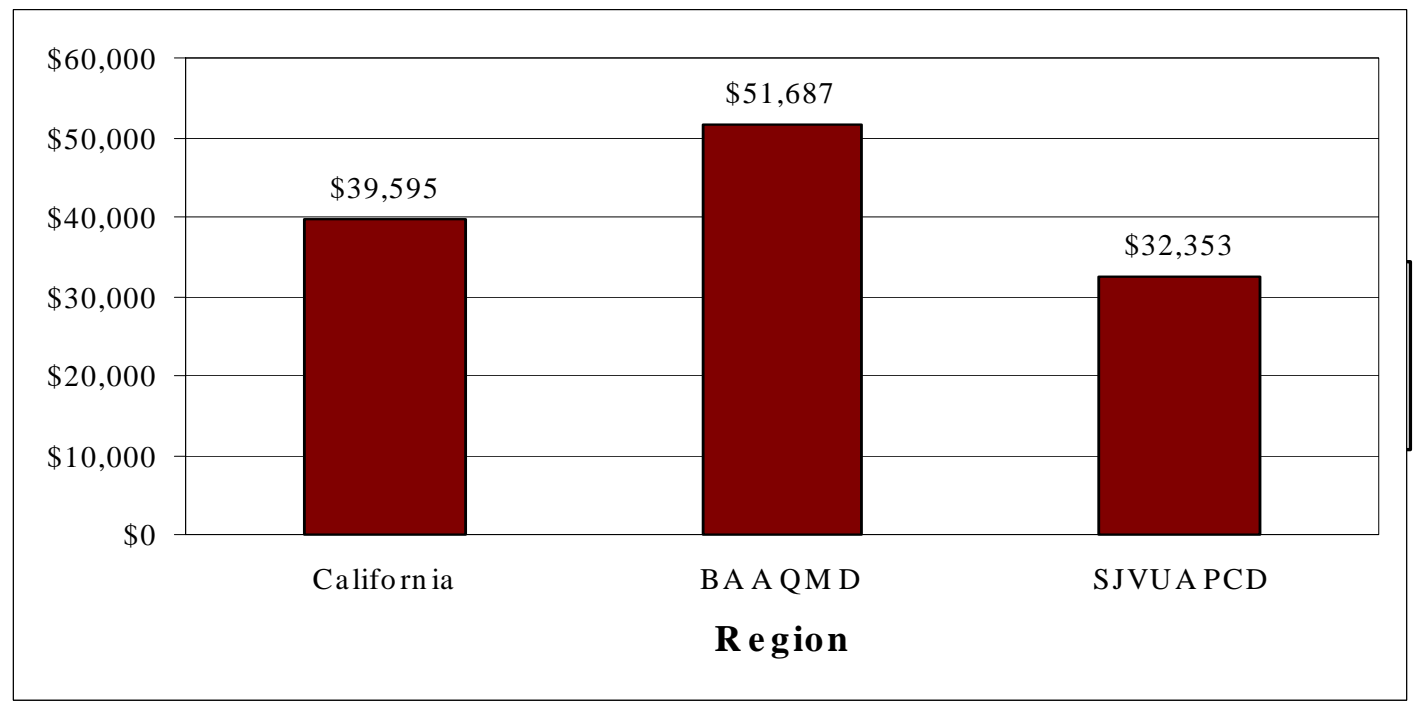

Figure APPC-9: Persons Living Below Poverty, 1997 Model-based (Census 2000)

${ }^{138}$ For a detailed definition, see U.S. Census website: http://www.census.gov/hhes/poverty/povdef.html. Last visited on April 16, 2003. 


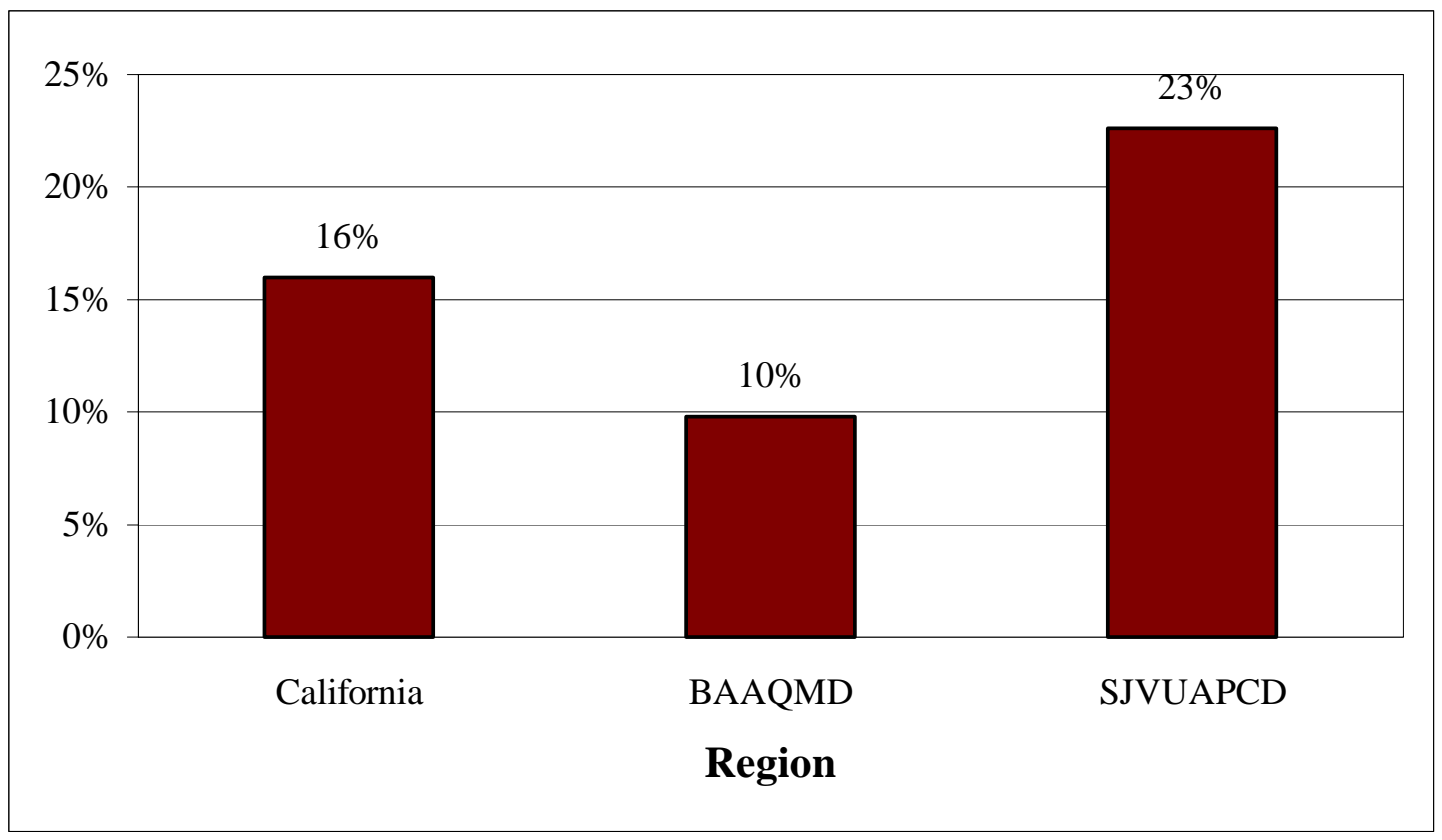

One economic study (Munroe et al. 2001) of the SJV found that real per capita income has declined since 1990 from $\$ 21,700$ to $\$ 20,900$ in 2000 (in 2000 dollars) and concluded,

Population-driven and cost-driven development activities that draw people and businesses to the [San Joaquin Valley] for the low cost of doing business and the low cost of living have created substantial economic growth through construction and retail jobs. Yet population-driven and cost-driving development have not created many of the high paying jobs that increase real per capita income (a key indicator of rising affluence in a region.)

Earlier in this chapter, population growth was associated with increasing pollutant emissions. Socioeconomic conditions also influence on emissions. Older cars emit more than new cars on a per-mile-driven basis (Calvert et al. 1993; Singer and Harley 2000). Poorer people will tend to have older autos (Singer and Harley 2000). The population of both people and cars is growing in the SJV. Relative to the Bay Area, the new SJV residents and their cars are poor in terms income and emissions performance. Another implication of poverty is that other planning issues, such as economic development, will 
overshadow air quality concerns, limiting regulatory and economic capacity, as well as political will, to address clean air.

The population of the SJV is also less educated than the Bay Area and California, as shown in Figures APPC-10 and APPC-11. 
Figure APPC-10: High School Graduates Per Capita (Calculation by Fine using Census 2000)

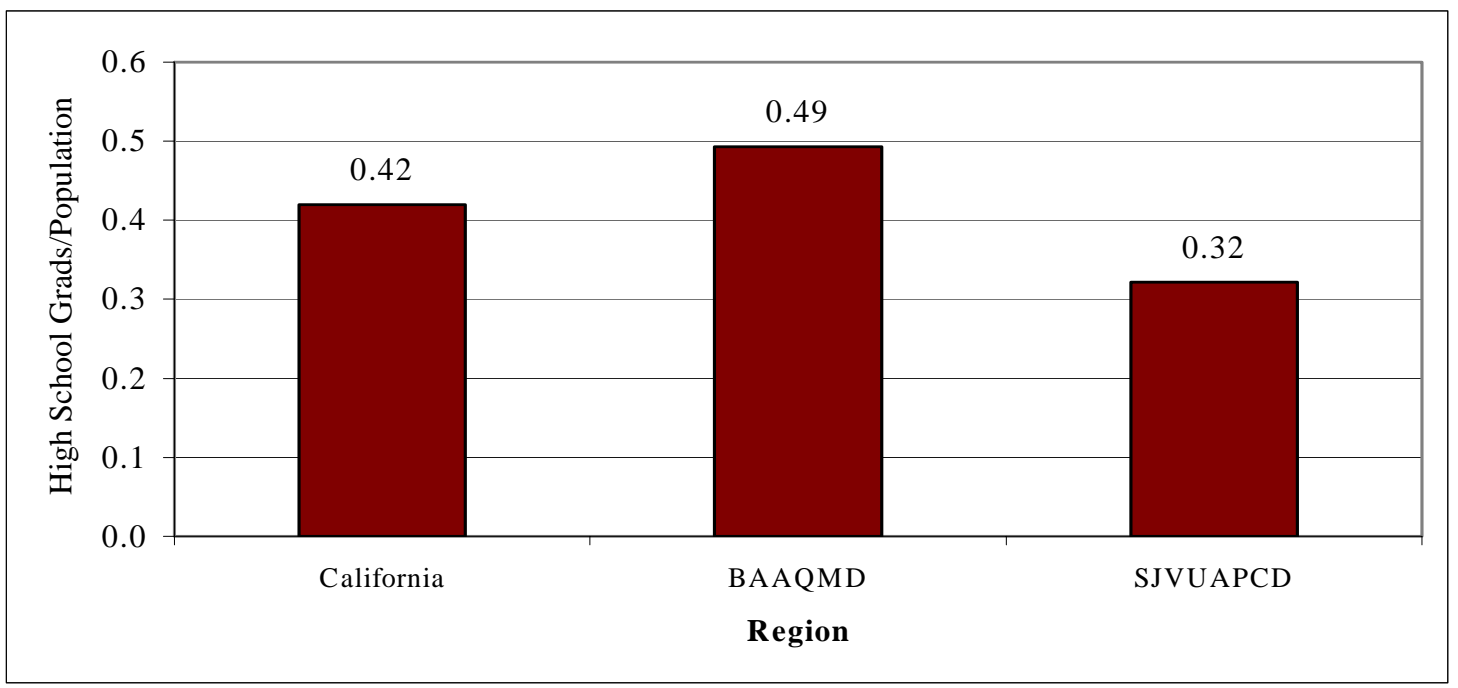

Figure APPC-11: College Graduates Per Capita

(Calculation by Fine using Census 2000)

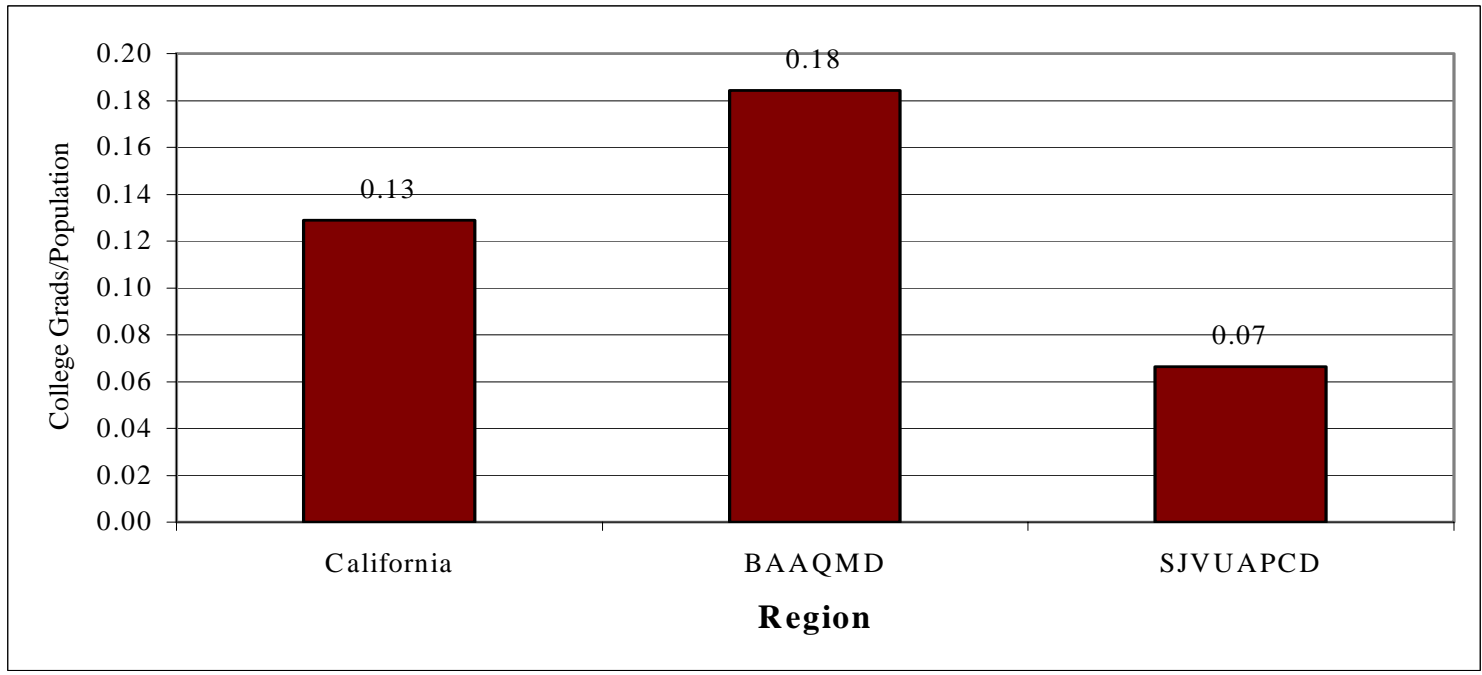

High unemployment adds to the SJV's socioeconomic concerns. The average unemployment in the SJV from 1990 to 1995 was 14.4 percent, almost double the California average of 7.8 percent (Penbera 1996). The trend continued in the later half of the decade, as shown in Figure APPC-12. 
Figure APPC-12: Unemployment in the SJV, California and U.S., 1990-2000 (Munroe et al. 2001)

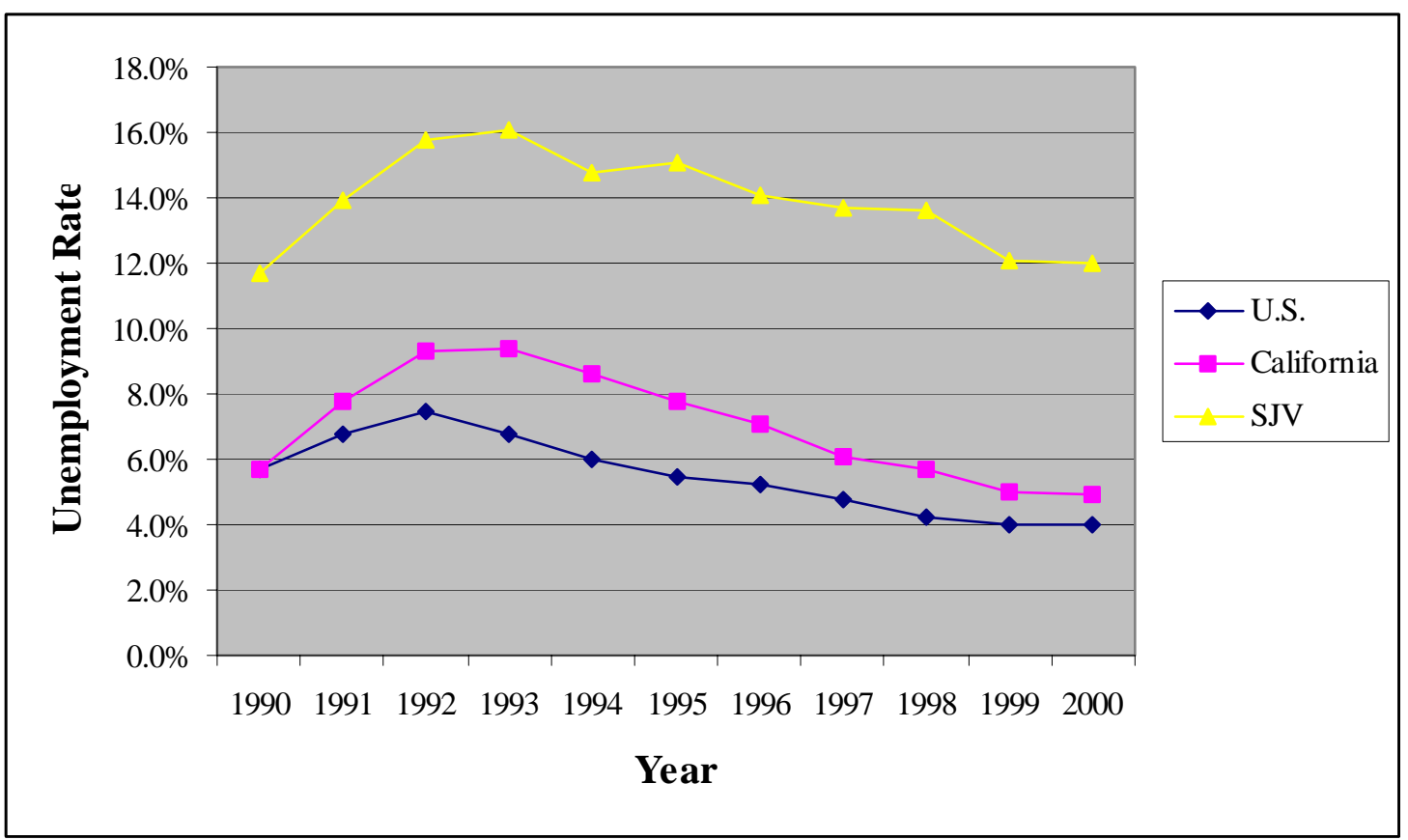

\section{Sources of Employment}

The need for and sources of employment in Central California informs discussion of air quality management regarding:

- Need for and willingness to require emissions controls on activities that generate employment.

- Veracity and relevance of the jobs versus the environment dichotomy.

- $\quad$ Tolerance for polluting industries that provide other benefits, such as employment.

- $\quad$ Capacity for the local economy to fund emissions controls.

The SJV is home to small and large-scale farming, and petroleum extraction and processing, as well as major metropolitan centers, such as Fresno, Bakersfield, Modesto, Stockton and Visalia. 
The agricultural and petroleum industries are considered very important to the SJV economy. They are of interest to this case as important sources of pollutant emissions. Agriculture in the SJV accounted for half of California’s \$25 billion agricultural output in 1995, and 17 percent of the region’s jobs are on farms (Penbera 1996). Another estimate of employment in the SJV for 2000 confirmed this percentage, but noted that employment in other sectors may also be due to agriculture (Munroe et al. 2001). For example, in San Joaquin County, one-third of all manufacturing jobs were attributed to agricultural-related food processing. Valley-wide, manufacturing jobs represent approximately 10 percent of all employment. As shown in Figure APPC- 13, SJV counties are more reliant on farming than other Californian counties.

Figure APPC-13: Farm Jobs as Percentage of Total Jobs By County

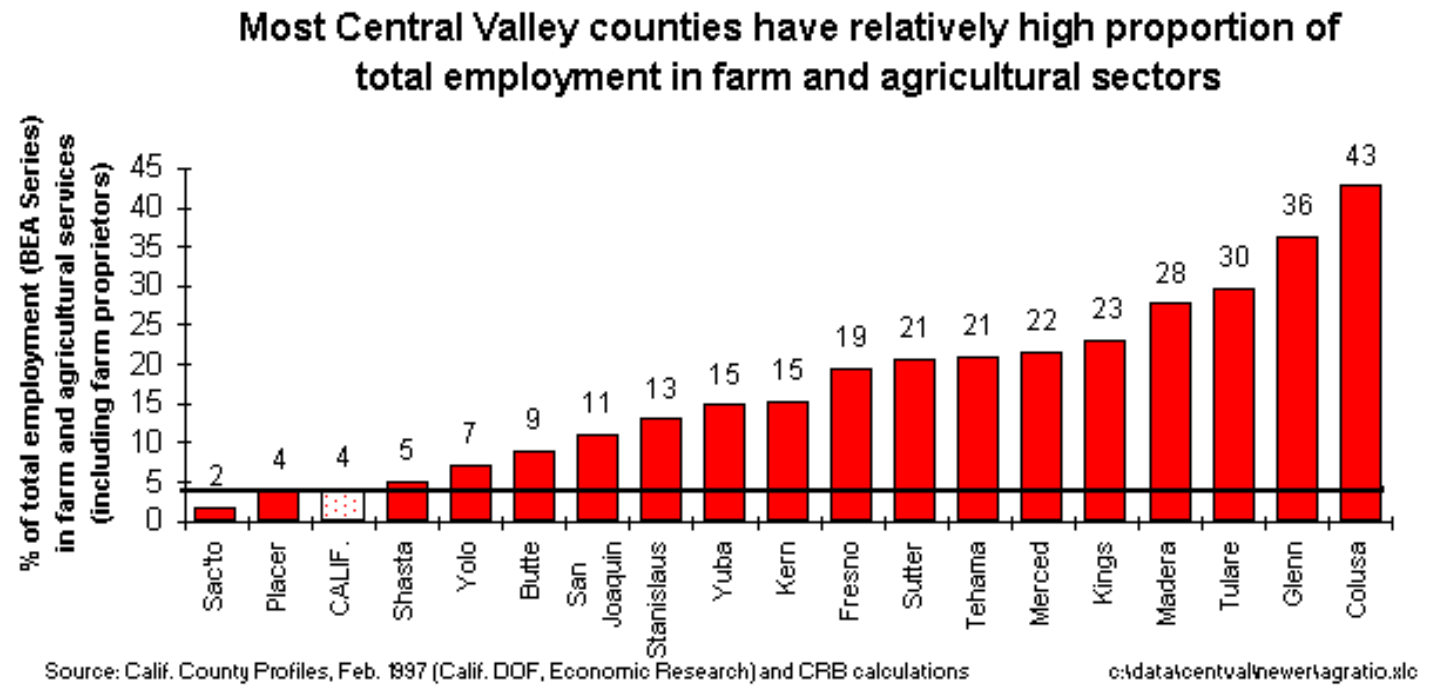

A comprehensive assessment of the economics of agriculture in the SJV is provided by the University of California Agricultural Issues Center. Their report estimates that 
agriculture represented 32 percent of total income (\$20 billion) and 37 percent of the jobs (1.3 million) in the SJV (Kuminoff et al. 2000). It is important to note, however, that these estimates are based on modeling. The report authors exercised IMPLAN, an input/output model developed by the U.S. Forest Service and U.S. Department of Agriculture. The uncertainty associated with the modeling is neither estimated nor discussed in the report.

Agricultural is an important source of pollutant emissions too, but has not been required to install pollutant emissions to the extent as the petroleum industry (EPA 2003). Agricultural emissions tend to be from non-point sources, such as farming equipment, windblown dust or livestock waste, that are harder to measure and monitor and may be harder to reduce than, for example, petroleum refinery smoke stacks.

The petroleum industry is also an important part of the SJV economy. Unlike agriculture, it is also a large part of the Bay Area economy too. No figures are available from independent sources to estimate the employment and income due to the petroleum industry in the SJV.

The WSPA sponsored a study of the importance of their industry in the Bay Area economy. It found that Bay Area refineries paid over \$105 million in local taxes and fees, including \$66 million for property taxes and \$10 million for regulatory and environmental fees (Quinn 2001). Relative to total tax revenues, however, refinery taxes and fees are not significant. For example, the $\$ 66$ million in property taxes is 1 percent of total payments of $\$ 6.2$ billion in the 9-county Bay Area in 1999 (BEQ 2002). 
Direct employment due to the petroleum industry was estimated to be 19,130 people in 1997, which ranks fifth amongst industrial employers. The top ranked industry, electronic components, employed over 75,000 in 1997 (Quinn 2001).

No studies of petroleum employment exist for the SJV but James Parsons, a cultural geographer, called petroleum the "kicker” in the regional economy (Parsons 1986). Indeed, Kern County is the most productive county in the United States, generating twothirds of all California petroleum. In 1999, oil wells in Kern County produced over 500,000 barrels of oil per day. The county ranks behind only Texas (1.4 million barrels per day), Louisiana (1.3 million barrels per day) and Alaska (1 million barrels per day) as the nation's most productive region.

Although oil production in Kern County has declined steadily since peak production of 424 billion barrels in 1985, it accounted 200 billion barrels or 68\% of the state’s total production in 2001 (Wilson et al. 2003). Furthermore, the county is estimated to contain 2.3 billion of the state's known oil reserves of 3.4 billion barrels (Wilson et al. 2003).

Using the same industrial classifications as Quinn (2001) to estimate petroleum industry employment in the Bay Area, an estimated 5,400 jobs in Kern County were directly petroleum-related in 1999. This does not include 2,000 jobs at service stations. It comprises only four percent of total employment in the county (Census 2000). Only six percent of total annual payroll, \$216 million, in 1999 was due to petroleum jobs. This does not seem to be a very significant part of the economy.

Petroleum is certainly a visible industry, as refineries and pumps appear to cover any part of the southern SJV not used for agricultural or urban purposes. Comparing the 
employment levels, for example, with the 6,000 employees of the Internal Revenues Processing Center in Fresno, puts the petroleum industry in perspective (Parsons 1986). It is not so important.

In addition to being employers and sources of income, farming and petroleum operations are sources of pollutant emissions. However, according to the WSPA study, only 4.0, 7.5 and 1.5 percent of reactive organic gases, nitrogen oxides, and particulate matter, respectively, emitted in the Bay Area are due to the petroleum industry (Quinn 2001). In Kern County, the estimate of emissions for 1999 indicates that petroleum is responsible for 80 and 60 percent, respectively, of all stationary source VOCs and NOx emissions, which is about one-third of emissions in the county (SJVUAPCD 2001). These estimates are examined in more detail in the Air Quality Setting chapter.

\section{Vehicle Miles Traveled}

There is a connection between population, employment and air quality. One obvious link is vehicular travel, since vehicular emissions are a large portion of regional emissions. Pollutant emissions associated with driving increase as the population drives more, either to work or for other purposes.

Total vehicle miles traveled (VMTs) increase with population growth. Though offset somewhat by the development of ever-cleaner autos and fuels, fuel consumption is another proxy for auto-related emissions. Figure APPC-14 shows that both VMTs and vehicle fuel consumption have and will continue to grow in the SJV and Bay Area. 
Figure APPC-14: Vehicle Miles Traveled, 1990-2020 (DOT 1999)

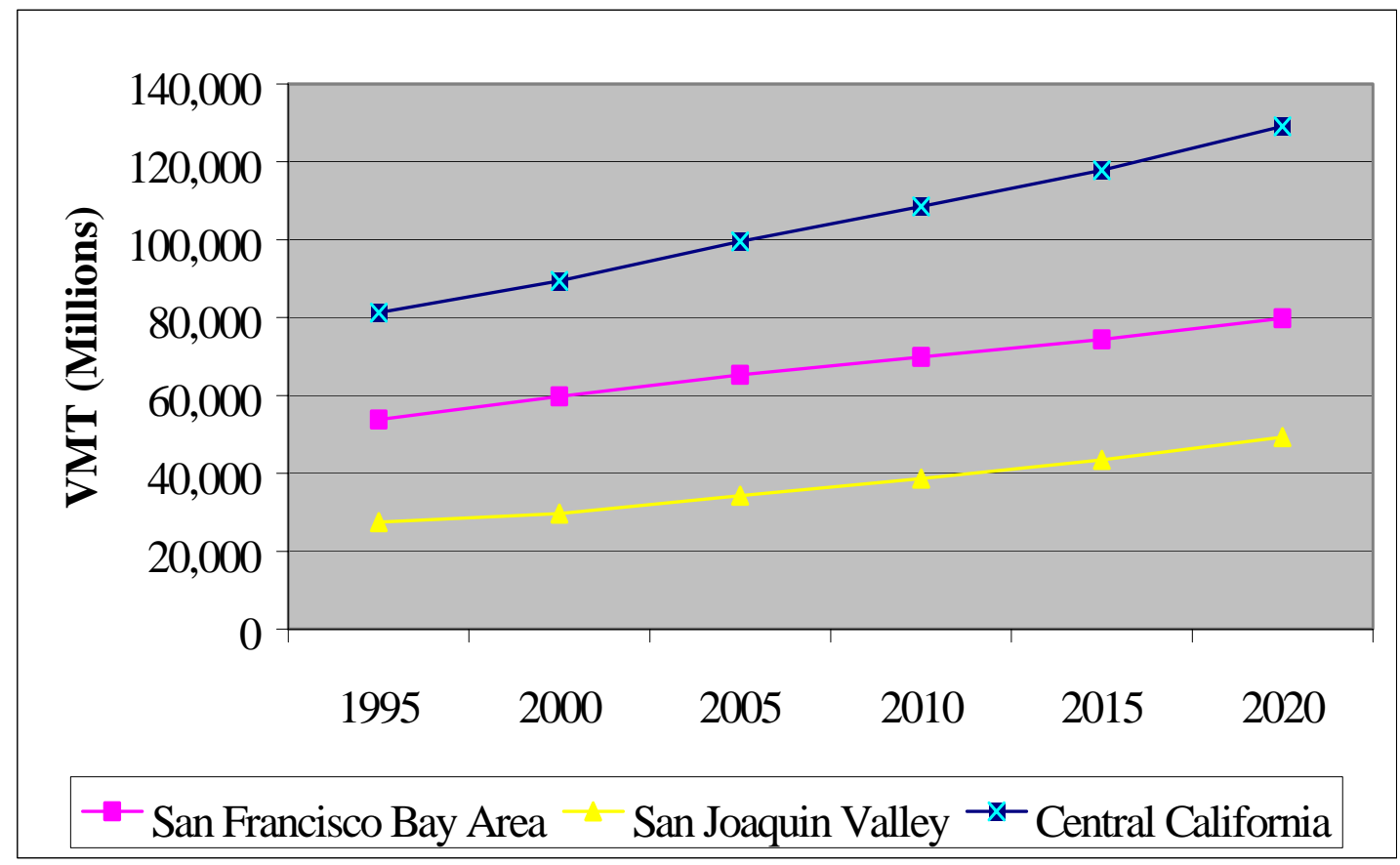

Figure APPC-15: Vehicle Fuel Consumption, 1990-2020 (DOT 1999)

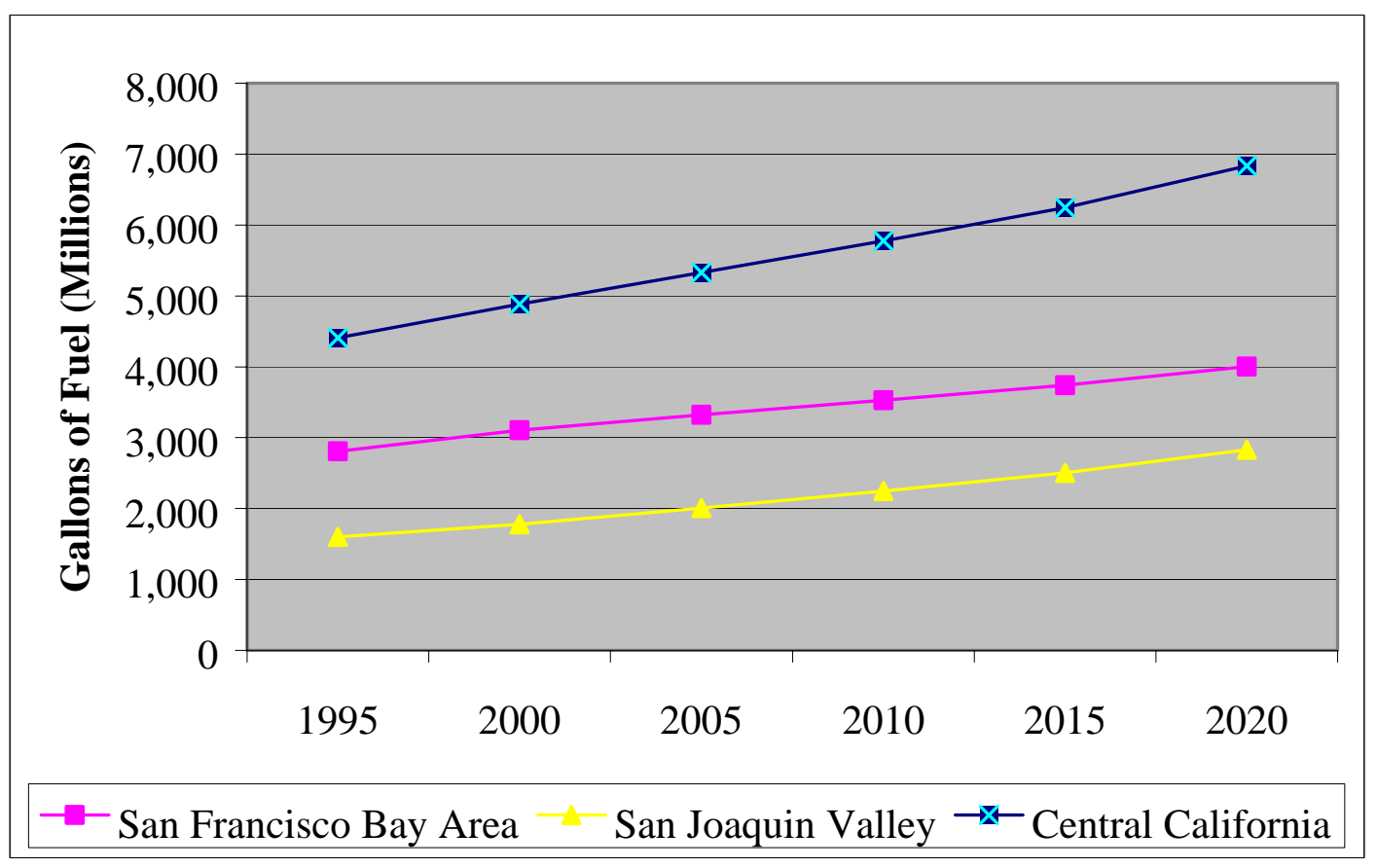




\section{Political Setting}

The region is an important "swing” vote, given that California’s other two major population centers, the S.F. Bay Area and Southern California, vote consistently democratic and republican, respectively (Baldassare 2001, page i). In 1998, registered voters were split evenly between democrats (42\%) and republicans (44\%) (Abstract 2001). Of the remaining 14\%, unaffiliated and registered indepedents were $10 \%$ and $2 \%$, respectively, with the remaining 2\% split between the Green, Reform, Libertarian, Natural Law, or other parties. Thus, the SJV receives considerable attention from politicians. For example, Governor Davis held an economic summit in the SJV soon after his election. In his speech at the summit, Governor Davis was perhaps referring to air quality ironically when he quoted William Everson saying "We saw time in the air" and lamented that we have not "fulfilled the economic promise of this great region" (Davis 1999).

In one breath, Governor Davis captured the political and economic tone of the SJV. Although vibrant and growing, socioeconomic woes and environmental concerns remain. Governor Davis offered several new initiatives:

- Speed the opening of the new University of California Merced campus

- Invest in biomass energy production

- Bring the Internet to more homes, including rural residents

- Improve transportation

Though the new U.C. campus in Merced will spur growth in the region, each of these initiatives impacts air quality. Telecommuting via the Internet reduces motor vehicle 
emissions. So too do transportation improvements, if they reduce congestion without increasing fuel consumption.

Like so many politicians, Governor Davis recognizes the importance of voters in the SJV, and has taken direct steps to gain their support. Presidential and Gubernatorial elections are not the only way state and federal politics are influenced by local politics in the SJV.

Congressional representatives also engage in air quality planning debates. For example, Congressman Gary Condit, whose constituency was in the SJV, wrote to EPA Region IX to express concern about "a lack of good faith on the part of the BAAQMD to work closely with the SJV on constructive solutions to the problem of [air pollutant] transport” (Condit 1996).

There are at least two very well organized political lobbies in the SJV. The petroleum industry has two active industry associations, WSPA and Independent Oil Producers Agency (IOPA), actively involved in air quality studies and planning. These associations support lobbyists at the state and federal level, as well as policy specialists and scientists engaged in local planning issues. For example, WSPA and IOPA contribute funds for the Central California air quality studies and, consequently, have representatives on the Policy and Technical Committees overseeing those studies. Furthermore, these associations champion other groups, such as the California Council for Economic and Environmental Balance, who share their philosophies about pursuing economically efficient solutions to environmental concerns. Other representatives of the 
regulated community, also with seats on the Policy Committee, include the California Trucking Association and the Electric Power Research Institute.

The greatest economic force in the SJV is the agricultural industry. Some of them actively commenting on air quality planning decisions include:

- Almond Hullers and Processors Association

- California Citrus Mutual

- California Cotton Ginners and Growers Association

- California Farm Bureau Association

- California Grape and Tree Fruit League

- County Farm Bureaus

- County Water Districts

- Nisei Farmers League

Like petroleum interests, agriculturalists are involved at the local, regional, state and national levels. However, unlike petroleum, they have few air quality scientists representing them. This is no surprise considering that the petroleum industry has long been engaged in air quality planning and emissions controls, whereas agriculture has been largely exempt from emissions controls. Although fugitive dust emissions were implicated by planners in the SJV pursuing the PM ${ }_{10}$ NAAQS (SJVUAPCD 1997), controls on emissions of pollutants that form ozone, namely nitrogen oxides and hydrocarbons, have focused mostly on the big stack industries, like petroleum refiners and power plants, and little moving pipes, motor vehicles. ${ }^{139}$ As discussed in the next

\footnotetext{
${ }^{139}$ Actually, it is not accurate to consider autos to be little moving pipes. They emit while idling and
} 
chapter, there is now clear impetus to control agricultural pollution to meet the ozone standard in the SJV. Up to now, however, no such controls have been implemented.

Air districts other than the SJVUAPCD and BAAQMD and environmental groups also deserve mention. They are notable for their relative impotence compared with the powerful agricultural and petroleum lobbies. Air districts such as the Sacramento Metropolitan Air Quality Management District are small, relatively new, and possess limited technical capabilities. They comment on air quality plans produced for the SJV and Bay Area, motivated largely by concerns that pollutant emissions transport between districts.

Finally, a group notable for its absence in the SJV is the environmental coalition. No active, organized, local environmental advocacy groups were engaged in air quality planning debates in the SJV during the mid-1990's. The Bay Area, however, is at the other end of the spectrum. It is home to a long list of very active local and national environmental advocacy groups. Notwithstanding the Sierra Club’s Bay Area Chapter, national advocacy groups, such as Greenpeace, Environmental Defense, and Natural Resources Defense Council, do not participate in local air quality planning efforts. Other groups, such as Citizens for a Better Environment and Greenaction, however, are quite involved. Other environmental advocacy groups actively engaging Bay Area air quality planning include TransDef, Our Children’s Future, and the Golden Gate Law School Environmental Justice Clinic. These groups mobilize local citizens to demonstrate at 
public hearings and to comment on draft air quality plans. The Western States Petroleum Association described their participation as follows,

Several San Francisco Bay Area environmental groups demonstrated against local refineries and power plants in 2001, primarily objecting to the Ozone Attainment Plan developed by the Bay Area Air Quality Management District. During the demonstrations, Communities for a Better Environment called for unreasonable and unnecessary emission controls on flares, tanks and wastewater systems. (WSPA 2001)

The environmental advocates did not deem emissions controls unnecessary or unreasonable. The demonstrations raise questions about the planning process and why it is that at least some people feel so frustrated with the process, unable to be heard and unsatisfied, that they resorted to public protest. Or, why it is that a similar position never coalesced to the point of organized protest in the SJV in the 1990's.

\section{Planning Challenges in the SJV}

\section{Socioeconomics}

The previous section describes the different socioeconomic conditions in the SJV by comparison with the Bay Area and California as a whole. The Bay Area is rich, educated, healthy and diversified, whereas the SJV is poor, unemployed, less educated, less healthy, and more dependent on agriculture for employment. Consequently, socioeconomic well-being is a fundamental underlying planning challenge in the SJV.

The Central Valley Survey in 2001 was a joint effort by the Great Valley Center and the Policy Institute of California to interview over 2,000 adults in the Central Valley. When asked about the most important issue facing the region, population growth and the "electricity crisis" each received 15 percent of the votes, jobs and the economy was a close third with 13 percent, and water was fourth with 8 percent (Baldassare 2001). The 
same survey found that 26 and 38 percent believed air quality was a "big problem” and “some problem”, respectively. Furthermore, more than half of the respondents believe that air and water quality conditions posed serious threats to their own health and wellbeing (Baldassare 2001, page vi). However, as show in Table APPC-C, problems of job and farmland loss were concerning to a greater percentage of the respondents that was air quality, but air quality was a close third.

Table APPC-C: List of Local Concerns of Surveyed Central Valley Residents (Baldassare 2001)

\begin{tabular}{|l|c|}
\hline Concern & $\begin{array}{l}\text { Respondents stating concern was at least } \\
\text { “Some Problem” }\end{array}$ \\
\hline Well-paying jobs & $73 \%$ \\
\hline Loss of farmlands & $67 \%$ \\
\hline Air pollution & $64 \%$ \\
\hline Population growth and development & $63 \%$ \\
\hline Traffic congestion & $63 \%$ \\
\hline Affordable housing & $59 \%$ \\
\hline
\end{tabular}

\section{Natural Resources}

Air quality is the planning concern of primary focus in this case, so it is discussed in great detail in the next chapter. Generally, natural resources pervade planning concerns in the SJV. A report by the Great Valley Center, The State of the Central Valley (GVC 1999), assessed energy consumption, solid waste generation, and endangered species habitat conservation. It concluded that per capita energy consumption in the SJV is about one-third higher than the rest of the California, that solid waste diversion to recycling centers will not meet targeted rates, and that the San Joaquin Valley is not getting its share of habitat conservation funds . Although it was not addressed directly in the Central Valley Survey, water allocation is another important concern in the SJV related to its resource base, as this banner appearing along at least two major roadways attests: 


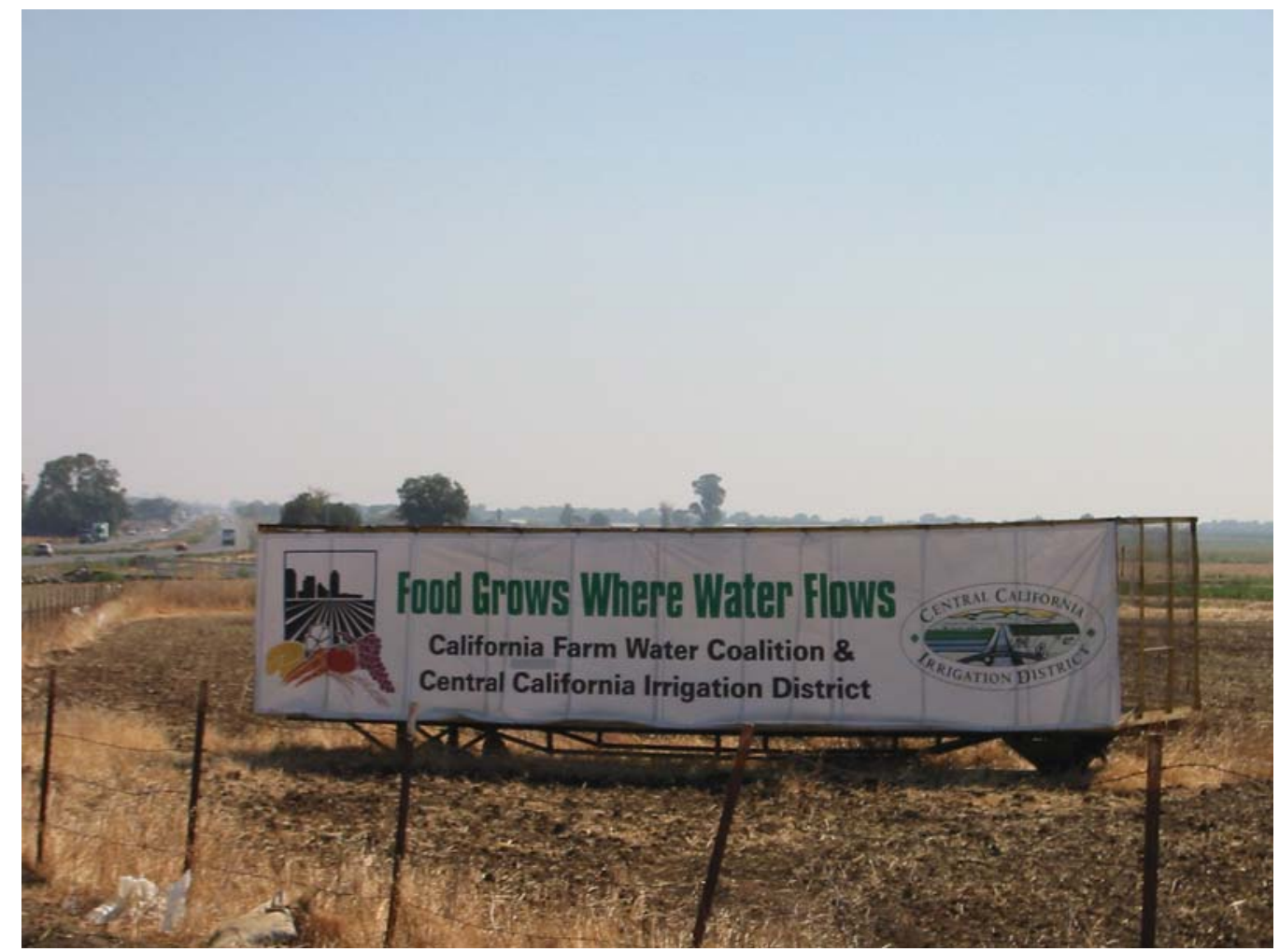

\section{Air Quality}

There are different opinions about air quality, both as a governing concern to planners and with respect to the political and public will to address it. In 1986, Mr. James Parsons, a cultural geographer studying the SJV, commented,

A pro-business climate has meant minimal zoning standards and pollution controls, but for how long? An early morning veil of brown smog lays low on the Fresno horizon with increasing frequency. During rush hours air traffic patrols direct commuters to the local radio. High ozone counts are suspected of reducing cotton yields and damaging the lower Sierra forests. The greatest lure continues to be the availability of land. The real boom may yet be several years away (Parsons 1986).

Fifteen years later, the SJV continues to experience the "boom”. It also has more information about the air quality conditions. Nonetheless, Dr. James Boyd, former Executive Officer of ARB and currently Chief of Staff at the California State Resources Agency, said, 
Today I don't see as much interest in regulatory intrusion. There's a lot of people who are new in the Legislature, and they seem to think California has a great [air quality] program, and they don't need to dirty their hands with it (Polakovic 2001).

Air quality conditions and trends are discussed at in the following chapter. Currently, the SJVUAPCD is charged with producing a new plan that lays out a plausible course for meeting the federal ozone standard by November 15, 2005. These requirements and deadlines were triggered when the U.S. EPA officially determined that the SJV air basin (SJVAB) failed to achieve the ozone standard by the FCAA's November 15, 1999. Consequently, the SJVAB was reclassified from a "serious" to a "severe" nonattainment area for ozone (Fed. Reg. 2001). ${ }^{140}$ In addition, the SJVAB is classified as a serious nonattainment area for particulate matter.

In studying air quality conditions in its air basin, the SJVUAPCD has concluded that it cannot clean up the air by the 2005 deadline, stating,

Preliminary modeling results completed by ARB and District staff has estimated that emissions reductions of at least 150 tons/day for both VOCs and $\mathrm{NO}_{\mathrm{x}}$ will be required to meet the new national ozone attainment deadline. To be forthright with the public, this amount of emission reductions represent an impossible task...(SJVUAPCD 2001)

Consequently, the SJVUAPCD is considering the decision to voluntarily reclassify itself as "extreme" nonattainment for the SJV air basin, which will, amongst other things, push back the deadline for attainment to November 15, 2010. In discussing this decision, the SJVUAPCD wrote,

The SJVAB is in a very precarious position in balancing the mandate of the federal Clean Air Act, citizen demands for a healthy environment while maintaining a viable economic base that will accommodate the clean air program costs, and the Valley's projected population growth...

\footnotetext{
${ }^{140}$ These classifications and their implications are described in more detail in the previous chapter, Central California Setting.
} 
Voluntary reclassification to an Extreme Nonattainment Area would be a bold decision by the Governing Board, and it would require substantial buyin from city and county governments, citizens of this Valley and the various industrial processes that would be directly impacted. It will require concerted effort by all affected parties of this Valley to embrace the selected course of action. (SJVUAPCD 2001)

It remains to be seen if such buy-in is possible in the SJV.

\section{E. Summary and Conclusions}

This chapter provides an overview of the geography of Central California, notably the San Joaquin Valley, as well as the region's socioeconomic and demographic conditions. The Bay Area and the SJV are compared to reveal the relative importance of and ability to respond to air quality concerns. The comparison is also relevant because the Bay Area is an upwind contributor of air pollution to the SJV. The chapter concludes with a discussion placing air quality control in perspective relative to other planning concerns in the SJV. The main points of this chapter are:

- The geography and meteorology of the SJV are ideal for capturing, retaining and forming ozone and particulate matter air pollution.

- "Central California" is defined by the modeling domain used for ozone air quality studies of the SJV. The air districts of primary interest are the Bay Area Air Quality Management District and the San Joaquin Valley Unified Air Pollution Control District. They are responsible for the San Francisco Bay Area and San Joaquin Valley Air Basins, respectively.

- Atmospheric transport connects the Bay Area and SJV. Due to prevailing westerly wind, the Bay Area contributes ambient pollution to the SJV. However, it is difficult to know the magnitude or significance of transport. Modeling is the primary tool used to assess the role of upwind emissions (e.g., Bay Area) on downwind (i.e., SJV) air quality.

- The SJV population has grown faster more recently than the Bay Area. However, growth rates are deceptive since the Bay Area is twice as populous as the SJV. The population of the SJV is projected to more than double by 2040. 
- The population of the SJV is a minority majority and is increasingly minority. They are also are poorer, less educated, and less employed than residents in the Bay Area.

- The SJV is an important "swing” vote in California, since southern and northern California vote consistently Republican and Democratic, respectively. About 80 percent of the voting population in the SJV is split between Democrats and Republicans, so politicians must give the region considerable attention to win state-wide elections.

- Agriculture is the most significant revenue and employment sector in the SJV. Petroleum extraction and refining is considered important to both the Bay Area and SJV economies, however employment and income numbers do not necessarily support this conclusion.

- Given the socioeconomic and demographic conditions in the SJV, it remains to be seen if there is adequate capacity and public sentiment to take the steps necessary to meet air quality standards. Unemployment, population growth, land use conversion, and water resources are the foremost issues in the SJV. Although air quality is a concern, it may not be a priority relative to other planning challenges.

The major findings of this chapter reveal how the San Francisco Bay Area and the SJV differ socially, economically and physically. These differences have bearing on both air quality conditions and potential responses. Considerable growth is anticipated for the SJV, which also means that emissions-producing activities, such as driving, will grow too. Furthermore, poor economic conditions in the SVJ relative to the Bay Area and the rest of California will affect the region's ability to respond to air quality concerns. It may even exacerbate them. For example, poorer people tend to drive older, dirtier cars. Thus, improved environmental quality may be undermined by a perception that it will come at the expense of economic vitality. Simply put, environmental quality may viewed by its residents as a luxury the SJV cannot afford. Air quality in the SJV may worsen before it gets better. 